Aaron Michael McGowan

333 words

\title{
Observation of deficit in NuMI neutrino-induced rock and non-fiducial muons in MINOS Far Detector and measurement of neutrino oscillation parameters
}

The MINOS (Main Injector Neutrino Oscillation Search) experiment has observed[1] muon neutrino disappearance consistent with the oscillation hypothesis tested by Super-Kamiokande[2][3] and K2K[4][5]. The survival probability for $\nu_{\mu}$ is given approximately by $1-\sin ^{2} 2 \theta_{23} \sin ^{2}\left(1.27 \Delta m_{32}^{2} \frac{L}{E}\right)$, where $\theta_{23}$ and $\Delta m_{32}^{2}$ are the mixing angle and difference in mass squared in $\mathrm{eV}^{2} / \mathrm{c}^{4}$ between the mass eigenstates $\nu_{3}$ and $\nu_{2}, L$ is the distance traveled in $\mathrm{km}$, and $E$ is the neutrino energy in $\mathrm{GeV}$. In the Near Detector at Fermilab, a measurement of the energy spectrum of the NuMI neutrino beam is made $1 \mathrm{~km}$ from the beam target. The neutrinos travel to the Far Detector in the Soudan Underground Laboratory, where another measurement of the energy spectrum is made $735 \mathrm{~km}$ from the target. MINOS measures $\left|\Delta m_{32}^{2}\right|$ and $\sin ^{2} 2 \theta_{23}$ by comparing the ND and FD neutrino energy spectra. In this dissertation, an alternate method is presented that utilizes rock muons, a class of events that occur when a $\nu_{\mu}$ interaction takes place in the rock surrounding the FD. Many muons that result from these interactions penetrate the rock and reach the detector. Muon events from $\nu_{\mu}$ interactions in the non-fiducial volume of the FD are also used in this analysis. The distribution of reconstructed muon momentum and direction relative to the beam is predicted by Monte Carlo simulation, normalized by the measured $\nu_{\mu}$ energy spectrum at the ND. In the first year of NuMI running (an exposure of $1.27 \times 10^{20}$ protons on target) 117 selected events are observed below $3.0 \mathrm{GeV} / \mathrm{c}$, where $150.2 \pm 16.1$ events are expected. When a fit is performed to events below $10.0 \mathrm{GeV} / \mathrm{c}$, the null (no disappearance) hypothesis is ruled out at significance level $\alpha=4.2 \times 10^{-3}$. The data are consistent with the oscillation hypothesis given parameter values

$$
\left|\Delta m_{32}^{2}\right|=2.32 \pm_{0.75}^{1.06} \times 10^{-3} \mathrm{eV}^{2} / c^{4} \text { (stat+sys) and } \sin ^{2} 2 \theta_{23}>0.48 \text { (68\% C.L.) }
$$

which is in agreement with the published MINOS result

$$
\left|\Delta m_{32}^{2}\right|=2.74 \pm_{0.26}^{0.44} \times 10^{-3} \mathrm{eV}^{2} / c^{4} \text { (stat+sys) and } \sin ^{2} 2 \theta_{23}>0.87 \text { (68\% C.L.). }
$$

Faculty Advisor's Signature 


\title{
UNIVERSITY OF MINNESOTA
}

This is to certify that I have examined this copy of a doctoral dissertation by

\author{
Aaron Michael McGowan
}

and have found that it is complete and satisfactory in all respects, and that any and all revisions required by the final examining committee have been made.

Prof. Marvin L. Marshak

Faculty Advisor

Signature

Date

GRADUATE SCHOOL 


\title{
Observation of deficit in NuMI neutrino-induced rock and non-fiducial muons in MINOS Far Detector and measurement of neutrino oscillation parameters
}

\author{
A DISSERTATION \\ SUBMITTED TO THE FACULTY OF THE GRADUATE SCHOOL \\ OF THE UNIVERSITY OF MINNESOTA \\ BY
}

Aaron Michael McGowan

B.S., Cornell University, 2003

IN PARTIAL FULFILLMENT OF THE REQUIREMENTS

FOR THE DEGREE OF

DOCTOR OF PHILOSOPHY

Prof. Marvin L. Marshak, Faculty Advisor

School of Physics and Astronomy

Dr. Maury C. Goodman, Co-Advisor

Argonne National Laboratory 
(C) Aaron Michael McGowan 2007 


\section{Acknowledgements}

I would like to thank the following people, without whom I could not have completed this work, I might be traveling a different career trajectory, and I may have lost my mind:

My advisor, Marvin Marshak, for his continual advocacy of my peculiar way of life in graduate school. I met Marvin during my REU in Minnesota the summer before I graduated from Cornell, and he pitched MINOS magnificently. We shared stories of life in the (now extinct) Class Halls and anecdotes from our experiences on Libe Slope. Marvin pushed me quickly through the graduate curriculum and sent me to Argonne, where I could be close to the daily operations of our experiment. Most of all, Marvin understood my plight of falling in love with my best friend from college, who went on to medical school in Manhattan. He made our long-distance relationship possible, and has rendered me nearly as much relationship advice as guidance for my analysis.

My advisor away from the university halls, Maury Goodman, for the painstaking (painful?) editing of this document and for daily filling the gap that exists in public transportation between Fermilab and Argonne. Maury helped me to boil problems down to simple arguments, making it clear to me when I was fixated on a second-order effect. I also thank Sharon Goodman, for providing delicious home-cooked dinners on occasion as a break from the weary road of ramen noodles.

All MINOS collaborators who came before me. I came into MINOS roughly twelve years after it formed. It goes without saying that none of the work in this dissertation would be possible without the years of dedicated service that have gone into this experiment by the past and present members of our collaboration.

The mine crew: Bill Miller, Jerry Meier, Dave Saranen, Eileen Amos, Doug Wiermaa, Brian Anderson, and Curt Lerol, for introducing me to the way of life in the north country, half a mile underground.

The Minnesota Department of Natural Resources and the Soudan Underground Mine 
State Park for hosting our experiment.

Pete Border, Leon Mualem, and Keith Ruddick, for introducing me to the clumsy art of experimentation and preparing me for research in a no-nonsense way.

The neutrino group at Argonne: Juergen Reichenbacher, Tom Fields, Dave Ayres, Dave Reyna, and Phil Schreiner, for suggestions for this analysis and providing feedback on my presentations.

My friends in Dorm 6: Dan, Allison, Jim, and Rebekah, for introducing me to karaoke night and more free food than a grad student could wish for. I've never seen so many ribs cooked in one kitchen. Thanks for the euchre and hospitality.

All of Young MINOS, for the support, sounding board, and several evenings spent in diversion from the life that is graduate school.

My classmates from the Twin Cities: Pearl Sandick, Matt Fritts, Scott Thaller, Marie Lopez del Puerto, Charlie Blackwell, Beth Lusczek, Jason Haupt, Abe DeBenedetti, Hannes Hubmayr, Mun Chan, Brian Batell, and Nate Lindquist, for trips to the Village Wok, for bearing with me the huge burritos late at night, and for Poor Man's Black Velvet parties. You guys got me through two years of endless problem sets.

The older, wiser Minnesota students who helped me along the path: Ben Speakman, Emily Maher, Sujeewa Kumaratunga, Dipu Rahman, Jeremy Gogos, Bernie Becker, and Erik Beall.

My friends from AEP, Pete, Tom, Erin, Punit, Sergei. I kept the crafts in line, it was easy as 1-2-3, and I loved every minute of it. DP Dough sustained us late at night, studying for exams. We should make a trip back to the portal one of these days.

Ron Rezmer, for helping me recover what we could from my hard drive crash. Eve Kovacs for all her help administering the minossoft distribution at Argonne.

My buddies from high school, Dale Height, Jon Rustebakke, and Chris Thatcher. I never considered firing potatoes from PVC cannons or saran-wrapping an entire school bus until I met you guys. Our canoe trips were the highlight of my summers.

Steven Macks, for appreciating physics in the way I appreciate mathematics, for introducing me to the proper way one should relax after a bad day, and teaching me shortcuts to learning bass. I'll never look at the bread aisle the same way again.

Mr. Glen Golden, for starting up the AP Statistics course at Kennedy High School and taking your lunch hour to teach the first three students when administration tried to cancel the course for lack of enrollment. Thanks for teaching me about statistical significance and helping me to improve my golf swing. 
Rustem Ospanov, for introducing me to Interpol, Muse, The Pixies, and Blur during our night shifts together. The control room sound system sure got a workout.

Gavril Giurgiu for teaching me to relax in spite of research. Thanks for teaching me how to use LSF, ROOT, and Minuit. Thanks for picking me up in the rain when I tried to get from Fermilab to Argonne using only my feet and the spectacular public transportation in DuPage County. I enjoyed our outings with Vio and my introduction to a small slice of Romania. Noroc!

The Department of Energy and Oak Ridge Associated Universities, for supporting my trip to the 2005 Meeting of Nobel Laureates and Students in Lindau, Germany. It was truly amazing to meet Masatoshi Koshiba, David Gross, Frank Wilczek, Martinus Veltman, Douglas Osheroff, and other pioneers in physics, medicine, and chemistry. I was inspired by their work and by my fellow graduate students from around the world. Thanks to Lynette, Carol, and Randall for a fun week, your continued friendship, and a window into Singapore.

Charles Townes, the inventor of the laser, for conversations about resonant cavities and the Michelson-Morley-Miller experiments. I still believe the ether exists, just not as previously imagined.

My grandmother, Doris, who housed us when we first moved to Iowa, drove me and my sister to school in the mornings, and picked me up that time my car gave out on the interstate.

My grandparents, George and Phyllis, who bought me my first laser pointer when I was young and wasn't exactly sure what I would do with it (besides creating diffraction patterns on my bedroom wall). It now serves me well in seminars and lectures, and I used it yesterday to give my presentation in defense of this work.

My father, Michael, who always had faith in me throughout my academic career and helped to edit this document. I thank my mother, Ruth, who inspired me by starting a new career after raising two children. I thank my sister, Amy, who could defeat me in an argument any day - I look forward to watching your career in law develop. Seditious libel.

My wife, Sarah, who remains my best student in physics. Our time together kept me happy and healthy throughout graduate school, and I enjoyed learning enough of your trade to stay conversant at the dinner table. You bring out the best in me. I watched you grow from a pre-medical student to a resident, and I appreciate the work that went into your M.D. I am still amazed by the work of your hands, healing children day by 
day. The field of pediatrics just got more beautiful.

Almighty God, the Creator of everything seen and unseen. None of us will ever see a neutrino, but we can spend years uncovering their mysteries. Thank You for the opportunity to study this amazing universe.

$-\mathrm{AMM}$ 


\section{Dedication}

This work is dedicated to my Grandpa Dick, who used to drive ten hours with boat in tow to take us fishing for walleye on Kabetogama, instilling in me a love for the lakes of Minnesota. The many white polka dots on that green cap you gave me foreshadowed the billions of neutrinos that now illuminate the lake every two seconds. All these memories from photographs before kindergarten...I wish you could be here. 


\title{
Observation of deficit in NuMI neutrino-induced rock and non-fiducial muons in MINOS Far Detector and measurement of neutrino oscillation parameters
}

\author{
by Aaron Michael McGowan \\ Under the supervision of Prof. Marvin L. Marshak
}

\begin{abstract}
The MINOS (Main Injector Neutrino Oscillation Search) experiment has observed[1] muon neutrino disappearance consistent with the oscillation hypothesis tested by SuperKamiokande[2][3] and K2K[4][5]. The survival probability for $\nu_{\mu}$ is given approximately by $1-\sin ^{2} 2 \theta_{23} \sin ^{2}\left(1.27 \Delta m_{32}^{2} \frac{L}{E}\right)$, where $\theta_{23}$ and $\Delta m_{32}^{2}$ are the mixing angle and difference in mass squared in $\mathrm{eV}^{2} / \mathrm{c}^{4}$ between the mass eigenstates $\nu_{3}$ and $\nu_{2}, L$ is the distance traveled in $\mathrm{km}$, and $E$ is the neutrino energy in $\mathrm{GeV}$. In the Near Detector at Fermilab, a measurement of the energy spectrum of the NuMI neutrino beam is made $1 \mathrm{~km}$ from the beam target. The neutrinos travel to the Far Detector in the Soudan Underground Laboratory, where another measurement of the energy spectrum is made $735 \mathrm{~km}$ from the target. MINOS measures $\left|\Delta m_{32}^{2}\right|$ and $\sin ^{2} 2 \theta_{23}$ by comparing the ND and FD neutrino energy spectra. In this dissertation, an alternate method is presented that utilizes rock muons, a class of events that occur when a $\nu_{\mu}$ interaction takes place in the rock surrounding the FD. Many muons that result from these interactions penetrate the rock and reach the detector. Muon events from $\nu_{\mu}$ interactions in the non-fiducial volume of the FD are also used in this analysis. The distribution of reconstructed muon momentum and direction relative to the beam is predicted by Monte Carlo simulation, normalized by the measured $\nu_{\mu}$ energy spectrum at the ND. In the first year of NuMI running (an exposure of $1.27 \times 10^{20}$ protons on target) 117 selected events are observed below $3.0 \mathrm{GeV} / \mathrm{c}$, where $150.2 \pm 16.1$ events are expected. When a fit is performed to events below $10.0 \mathrm{GeV} / \mathrm{c}$, the null (no disappearance) hypothesis is ruled out at significance level $\alpha=4.2 \times 10^{-3}$. The data are consistent with the oscillation hypothesis given parameter values

$$
\left|\Delta m_{32}^{2}\right|=2.32 \pm_{0.75}^{1.06} \times 10^{-3} \mathrm{eV}^{2} / c^{4} \text { (stat+sys) and } \sin ^{2} 2 \theta_{23}>0.48 \text { (68\% C.L.) }
$$
\end{abstract}

which is in agreement with the published MINOS result

$$
\left|\Delta m_{32}^{2}\right|=2.74 \pm_{0.26}^{0.44} \times 10^{-3} \mathrm{eV}^{2} / c^{4} \text { (stat+sys) and } \sin ^{2} 2 \theta_{23}>0.87 \text { (68\% C.L.) }
$$




\section{Table of Contents}

Acknowledgements $\quad$ i

Dedication $\quad$ v

Abstract $\quad$ vi

List of Tables $\quad$ xi

List of Figures $\quad$ xiii

1 Introduction 1

1.1 Neutrino history . . . . . . . . . . . . . . . . 1

1.1.1 Discovery of the neutrino . . . . . . . . . . . . 3

1.1.2 The solar neutrino problem . . . . . . . . . . . . . 3

1.1.3 The atmospheric neutrino anomaly . . . . . . . . . . 4

1.2 Disappearance theories . . . . . . . . . . . . . . . . . . 5

1.3 Experimental tests . . . . . . . . . . . . . . 6

1.3.1 SNO confirms Standard Solar Model with total NC flux measurement 7

1.3.2 Super-Kamiokande measures atmospheric oscillation parameters . 7

1.3.3 K2K measures atmospheric parameters with accelerator neutrinos $\quad 7$

1.4 Rock muons . . . . . . . . . . . . . . . . . . . . . . . 8

1.4.1 Neutrino interactions . . . . . . . . . . . . . . . 8

1.4 .2 Upstream rock . . . . . . . . . . . . . . . . . . 10

1.4.3 Other experimental observations of rock muons . . . . . . . . 12

2 The MINOS experiment $\quad 13$

2.1 Historical development . . . . . . . . . . . . . . . . . 14 
2.2 Description of goals in context of rock muons . . . . . . . . . . . . . 15

2.3 The NuMI beam . . . . . . . . . . . . . . . . . . . . . . 16

2.4 The MINOS detectors . . . . . . . . . . . . . . . . . 22

2.4.1 Calibration Detector . . . . . . . . . . . . . . . 23

2.4 .2 Near Detector . . . . . . . . . . . . . . . . . . 24

2.4 .3 Far Detector . . . . . . . . . . . . . . . 25

2.4 .4 FD triggers . . . . . . . . . . . . . . . . . 27

2.5 FD event characteristics . . . . . . . . . . . . . . . . . . 28

2.5.1 Cosmic ray muon events . . . . . . . . . . . . . . . . 29

2.5.2 Contained vertex neutrino events . . . . . . . . . . . . . 31

2.5.3 Rock vertex muon events . . . . . . . . . . . . . . 33

2.6 Blind analysis criteria $\ldots \ldots \ldots \ldots$. . . . . . . . . . . . . . 33

3 Monte Carlo simulation $\quad 37$

3.1 Target and beam line . . . . . . . . . . . . . . . . . . . 38

3.2 Basic rock Monte Carlo . . . . . . . . . . . . . . . . . . . . . . 39

3.2 .1 Rock density . . . . . . . . . . . . . . . . 40

3.2 .2 Muon energy loss . . . . . . . . . . . . . . . . . . . . . 41

3.2 .3 Rock volume studies . . . . . . . . . . . . . . . . . . 43

3.2.4 Multiple Coulomb scattering . . . . . . . . . . . . . . 46

3.3 Soudan rock composition . . . . . . . . . . . . . . . . 46

4 Event selection $\quad 51$

4.1 Near Detector selection _. . . . . . . . . . . . . . . 52

4.2 Far Detector selection . . . . . . . . . . . . . . . 54

4.2.1 Inverse fiducial volume cuts . . . . . . . . . . . . . . . . . 54

4.2 .2 Rock muon cuts . . . . . . . . . . . . . . . . . . 58

4.2 .3 Hand scanning . . . . . . . . . . . . . . . . . . 59

4.3 Particle ID construction . . . . . . . . . . . . . . . . . 61

4.4 Efficiency and purity of event selection . . . . . . . . . . . . . 64

4.5 Backgrounds . . . . . . . . . . . . . . . . . . 67

4.5.1 Cosmic ray muons . . . . . . . . . . . . . . . 67

4.5.2 Neutral current interactions . . . . . . . . . . . . . . . 69

4.5.3 Beam-induced $\tau$ interactions leading to muon detection . . . . . 70 
5 Predicting the Far Detector event spectrum $\quad \mathbf{7 4}$

5.1 Near detector measurement of neutrino spectrum . . . . . . . . . . . 75

5.2 Extrapolation method . . . . . . . . . . . . . 77

5.2 .1 Correction for CC purity . . . . . . . . . . . 79

5.2 .2 Conversion from reconstructed to true energy . . . . . . . . . . 79

5.2 .3 Correction for selection efficiency . . . . . . . . . . . . 80

5.2 .4 Correction for slicing effects . . . . . . . . . . . . . . 82

5.2.5 Extrapolation using Far/Near flux ratio . . . . . . . . . . . . 83

5.2.6 Obtaining event weights from true $E_{\nu}$ prediction . . . . . . . . 85

5.2.7 Final reconstructed $P_{\mu}$ prediction . . . . . . . . . . . 86

5.3 Resulting muon predictions . . . . . . . . . . . 87

6 Mock data tests $\quad 90$

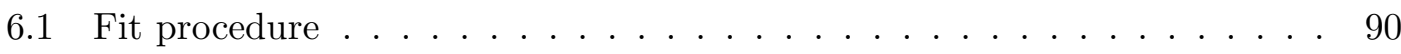

6.1.1 Rate, momentum, and direction . . . . . . . . . . . . 92

6.1 .2 Fit with penalty terms for normalization . . . . . . . . . 93

6.2 Mock data . . . . . . . . . . . . . . . . . . . 98

6.3 Normal flux . . . . . . . . . . . . . . . . . . . . . . 99

6.4 Tweaked flux . . . . . . . . . . . . . . . . 100

6.5 Precision of fit parameter measurements . . . . . . . . . . . . . . . . . . . . . . . . . . . . . . .

6.6 Systematic uncertainties . . . . . . . . . . . . . . . . . . 104

6.6.1 Normalization . . . . . . . . . . . . . . . . . . . . . 104

6.6 .2 Cross sections . . . . . . . . . . . . . . . . . 104

6.6 .3 Muon energy loss . . . . . . . . . . . . . . . . . . 105

6.6.4 Neutral current background . . . . . . . . . . . . . . . . . . . 105

6.6.5 Track momentum and angle . . . . . . . . . . . . 106

6.6 .6 Summary ........................ 106

7 Observed data and fit results 108

7.1 Event arrival time . . . . . . . . . . . . . . . 108

7.2 Comparison of reconstructed data to MC . . . . . . . . . . . . . . . . 109

7.2.1 Track vertex and endpoint location . . . . . . . . . . . . . 110

7.2.2 Track and shower fitting performance . . . . . . . . . . . . 112

7.2.3 Track momentum measurement . . . . . . . . . . . . . . . . . 114

7.2 .4 Particle ID distribution . . . . . . . . . . . . . . 116 
7.2.5 Two-dimensional momentum-direction histogram . . . . . . . . 116

7.3 Hypothesis test . . . . . . . . . . . . . . . . . . . . . 118

7.4 Fit results for oscillation parameters . . . . . . . . . . . . . 120

7.5 Comparison with published MINOS result . . . . . . . . . . . . . . 122

7.6 Comparison with other published experiments . . . . . . . . . . . . . 123

7.7 Future work . . . . . . . . . . . . . . . . . . . . 124

7.8 Conclusions . . . . . . . . . . . . . . . . . . . . . . . 125

Appendix A. MINOS Collaboration $\quad 129$

Appendix B. Physics derivations 131

B.1 Survival probability . . . . . . . . . . . . . . . . 131

B.2 Energy/angle . . . . . . . . . . . . . . . . 133

$\begin{array}{ll}\text { Appendix C. Statistical methods } & 137\end{array}$

C.1 Poisson $\log$ likelihood . . . . . . . . . . . . . . . . . . . 137

C.2 Chi-squared approximation ． . . . . . . . . . . . . . . 139

C.3 Feldman-Cousins method . . . . . . . . . . . . . . . . . . 139

Appendix D. Selected event list 142

$\begin{array}{ll}\text { References } & 151\end{array}$ 


\section{List of Tables}

3.1 Summary of particle decays in the NuMI beam line as modeled in GNuMI. 39

3.2 Cross reference for common Particle ID codes used in MINOS Monte Carlo. 48

3.3 Summary of simulated rock density changes in basic Monte Carlo. The calculated fluctuations in the muon rate are consistent with the statistical uncertainty (see Section 3.2.1) . . . . . . . . . . . . . . . . 49

3.4 Event rate for $1.27 \times 10^{20} \mathrm{POT}$ as a function of transverse rock thickness in Monte Carlo model. . . . . . . . . . . . . . . . . . . . . . . . . . 49

3.5 Comparison of composition between Greenstone and GMINOS rock. . . . 49

3.6 Composition of Greenstone rock, the main type found in the region surrounding the Soudan mine. . . . . . . . . . . . . . . . 5 50

3.7 Composition of rock as modeled in GMINOS simulation. . . . . . . . . . 50

4.1 Summary of event selection criteria and the events remaining after each cut. "MC Exp." displays the expectation for events in the antifiducial volume when combining the RV and CV MC with the proper $1.27 \mathrm{e} 20$ POT weighting and no oscillation. The closed data set has a rate reduced from the expectation consistent with oscillation. "Cosmic" displays the observed event rate from all backgrounds during a live time of 5.2 days in February 2006 (see Section 4.5). The expected number of cosmic ray muon events in the selected sample is $0.095 \pm 0.014 \ldots$. . . . . . . . 72

4.2 Summary of event selection criteria and the Monte Carlo events remaining after each cut - total events are scaled to the $1.27 \times 10^{20}$ POT dataequivalent exposure. "RV MC" displays the expectation for events modeled with an interaction vertex in the rock. "CV MC" labels those events modeled with an interaction vertex in the detector. . . . . . . . . . . 73 
6.1 Summary of cross section uncertainties and their effect on the fit parameters and the total expected event rates. . . . . . . . . . . . . 105

6.2 Summary of systematic uncertainties and their effect on the fit parameters and the total expected event rates. The total effect on each parameter is the sum in quadrature of the individual effects. . . . . . . . . . . . . 107

7.1 Best-fit values of the oscillation and normalization parameters obtained in final analysis, with statistical uncertainties. Units on $\left|\Delta m_{32}^{2}\right|$ are $\mathrm{eV}^{2} / \mathrm{c}^{4} .121$

D.1 Comparison of three selection decisions for all events used in the oscillation analysis. . . . . . . . . . . . . . . . . . . . 150 


\section{List of Figures}

1.1 Total differential neutrino charged current cross section arising from deep inelastic scattering, quasi-elastic scattering, and pion production. Figure taken from Lipari, Lusignoli, and Sartogo[23]. . . . . . . . . . . . . . 9

1.2 Ratio of neutrino energy spectra for rock muon events (RV) over contained vertex events $(\mathrm{CV})$ as modeled in Monte Carlo. The ratio follows the linear increase as expected from the extra power of energy present in the rock muon rate integral (Equation 1.3). . . . . . . . . . . . . 11

2.1 Cartoon diagram of the NuMI beam line at Fermilab (not to scale). . . . 17

2.2 Map showing the trip taken by NuMI neutrinos from Fermilab to Soudan. 18

2.3 Profile of the NuMI beam as it passes beneath Illinois, Wisconsin, and Minnesota. The central beam spot size is roughly $1 \mathrm{~km}$ wide at the Far Detector. . . . . . . . . . . . . . . . . . 19

2.4 Various NuMI beam configurations and their corresponding reconstructed neutrino energy spectra as observed with the MINOS Near Detector. Data is shown with black points, the original Monte Carlo in blue, and the tuned MC in red. LE-10 indicates the standard low-energy target position (retracted $10 \mathrm{~cm}$ from the edge of Horn 1). Standard horn current is $185 \mathrm{kA}$. The pME target location is retracted $1.0 \mathrm{~m}$ from the edge of Horn 1 , and the pHE target location is retracted $2.5 \mathrm{~m} \ldots \ldots \ldots$. . . . .

2.5 Neutrino event arrival times at the Near Detector. Figure taken from

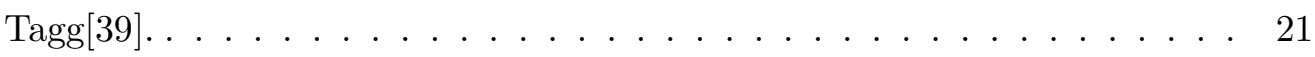

2.6 Overhead view of the MINOS Calibration Detector in place at CERN. The detector is now decommissioned. . . . . . . . . . . . . . . . . 24

2.7 Upstream view of the MINOS Near Detector. Note the human subjects for sense of scale. . . . . . . . . . . . . . . . . 2 25 
2.8 Downstream view of the MINOS Near Detector. Note the two electronics racks at the bottom of the view (about one meter tall) for sense of scale. . 27

2.9 Example of a cosmic ray muon event: Run 29349, Snarl 54101. . . . . . . 31

2.10 Example of a contained vertex CC DIS event: Run 32907, Snarl 102715. 32

2.11 Example of a selected rock muon event penetrating the front of the detector: Run 33452, Snarl 48800. . . . . . . . . . . . . . . . . . 34

2.12 Example of a selected rock muon event penetrating the side of the detector: Run 29349, Snarl 54101. . . . . . . . . . . . . . . . . 35

3.1 Muon energy loss in standard rock for energies below $120 \mathrm{GeV}$, dominated by the ionization term. . . . . . . . . . . . . . . . . . . 42

3.2 Muon energy loss in iron compared with loss in standard rock. . . . . . . 43

3.3 True neutrino interaction vertex locations as modeled in GMINOS for rock muon events passing Far Detector selection. The positive x-axis is into the page, the positive $y$-axis to the top of the page, and the positive z-axis to the right. Notice the upslope of the beam, which causes more muon events to enter the bottom of the detector. . . . . . . . . . . . . 45

4.1 Angular distribution of antifiducial muon events in GMINOS. . . . . . . . 57

4.2 Selected event with reconstruction problems: Run 32569, Snarl 91668. . . 61

4.3 Possible hadron shower event from neutrino interaction near front of cavern wall: Run 33184, Snarl 235339. . . . . . . . . . . . . . . . 62

4.4 Fraction of total event pulse height contained in reconstructed track. From left to right: Rock Vertex (RV), Contained Vertex (CV), and total events $(\mathrm{RV}+\mathrm{CV})$. Charged current distribution in black dashed line; Neutral current distribution in solid red line. All distributions are normalized to 1.0.

4.5 Average pulse height per plane for reconstructed track. From left to right: Rock Vertex (RV), Contained Vertex (CV), and total events (RV+CV). Charged current distribution in black dashed line; Neutral current distribution in solid red line. All distributions are normalized to 1.0. . . . . . . 64

4.6 Event length in number of planes. From left to right: Rock Vertex (RV), Contained Vertex $(\mathrm{CV})$, and total events $(\mathrm{RV}+\mathrm{CV})$. Charged current distribution in black dashed line; Neutral current distribution in solid red line. All distributions are normalized to $1.0 \ldots \ldots \ldots \ldots$. . . . . . . 
4.7 Particle ID parameter as calculated in Section 4.3. Charged current distribution shown as black dashed line; Neutral current distribution shown as solid red line. All distributions are normalized to 1.0 . . . . . . . . . 66

4.8 Charged current selection efficiency as a function of reconstructed muon momentum. Average values: 94.3\% (Rock Vertex), 86.3\% (Contained Vertex), 92.8\% (Combined). Note the different vertical scales. . . . . . . . 66

4.9 Charged current selection efficiency as a function of true neutrino energy. Average values: 91.4\% (Rock Vertex), 66.5\% (Contained Vertex), 87.7\% (Combined). Note the different vertical scales. . . . . . . . . . . . . 67

4.10 Charged current selection purity as a function of reconstructed muon momentum. Average values: 99.6\% (Rock Vertex), 98.9\% (Contained Vertex), $99.5 \%$ (Combined). Note the different vertical scales. . . . . . . . 68

4.11 Charged current selection purity as a function of true neutrino energy. Average values: 98.7\% (Rock Vertex), 93.9\% (Contained Vertex), 98.3\% (Combined). Note the different vertical scales. . . . . . . . . . . . . .

5.1 Maximum Detectable Momentum in the MINOS Near Detector. The dotted line indicates the median of the distribution. . . . . . . . . . . 77

5.2 Maximum Detectable Momentum in the MINOS Near Detector, divided into two track length categories. Short tracks are shown in the dashed black line, and long tracks are shown in the solid red line. . . . . . . . . . 78

5.3 Left: $\mathrm{CC} \nu_{\mu}$ purity as a function of reconstructed energy as modeled in Monte Carlo. Right: Selected CC-like events before (solid black line, "Reco CC-like") and after (solid red line, "Reco pure CC-like")the purity correction. . . . . . . . . . . . . . . . . . 80

5.4 Left: Reconstructed neutrino energy vs. true energy for true CC $\nu_{\mu}$ events. Right: Pure CC spectrum as reconstructed energy (solid red line, "Reco pure CC"), and after (solid black line, "True pure CC") correction to true

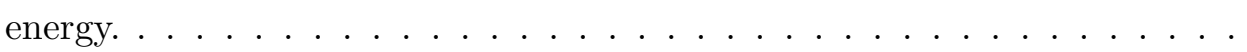

5.5 Left: selection efficiency as a function of true energy as modeled in Monte Carlo. Right: Measured spectrum at ND in true neutrino energy (solid black line, "True pure CC") and the actual spectrum (solid red line, "True pure CC corrected") after efficiency correction. . . . . . . . . . . . .

5.6 Weights applied to true neutrino energy spectrum to correct for double counting of events improperly sliced in time. . . . . . . . . . . . . . 
5.7 Far over near neutrino flux ratio used for extrapolation. . . . . . . . . . . 84

5.8 Near Detector data extrapolation. Left: True ND $\nu_{\mu} \mathrm{CC}$ event spectrum after the described processing steps; Right: Predicted True FD $\nu_{\mu} \mathrm{CC}$ event spectrum after extrapolation step. . . . . . . . . . . . 8 85

5.9 Ratio of predicted FD $\nu_{\mu} \mathrm{CC}$ event spectrum (using ND data extrapolation) to the input spectrum used in the nominal FD Monte Carlo. . . . . 86

5.10 Expected reconstructed muon momentum distribution for $1.27 \times 10^{20}$ POT. 87

5.11 Expected reconstructed muon direction distribution for $1.27 \times 10^{20}$ POT. $\quad 88$

5.12 Expected reconstructed muon distribution for $1.27 \times 10^{20}$ POT in twodimensional histogram of momentum and direction. . . . . . . . . . . . 89

6.1 Top row: oscillated (solid red line) and unoscillated (dotted blue line) MC spectra for parent neutrino energy (left), reconstructed muon energy (center) and direction (right). Bottom row: ratio of oscillated/unoscillated spectra for the three corresponding plots. . . . . . . . . . . . . . . 92

6.2 Selected Monte Carlo muon events below $60 \mathrm{GeV} / \mathrm{c}$, separated by those parented by pions and kaons. Pion contribution: dashed black line; kaon contribution: solid red line. . . . . . . . . . . . . . . . . . . . . 94

6.3 Selected Monte Carlo muon events below $30 \mathrm{GeV} / \mathrm{c}$, separated by those parented by pions and kaons. Pion contribution: dashed black line; kaon contribution: solid red line. . . . . . . . . . . . . . . . . . . 95

6.4 Parent neutrino spectra below $120 \mathrm{GeV}$ for muon events selected (up to momentum and direction cuts), separated by those parented by pions and kaons. Pion contribution: dashed black line; kaon contribution: solid red line. . . . . . . . . . . . . . . . . . . 96

6.5 Parent neutrino spectra below $60 \mathrm{GeV}$ for muon events selected (up to momentum and direction cuts), separated by those parented by pions and kaons. Pion contribution: dashed black line; kaon contribution: solid red line. . . . . . . . . . . . . . . . . . . . . . . 97

6.6 Parent neutrino spectra below $80 \mathrm{GeV}$ for muon events passing all selection cuts, separated by those parented by pions and kaons. Pion contribution: dashed black line; kaon contribution: solid red line. . . . . . . . . . . . . . 98

6.7 Parent neutrino spectra below $40 \mathrm{GeV}$ for muon events passing all selection cuts, separated by those parented by pions and kaons. Pion contribution: dashed black line; kaon contribution: solid red line. . . . . . . . . . . . . . 99 
6.8 Contours for mock data, normal flux. Light blue (inner), $\Delta \chi^{2}=1.0$; green (middle), 68\% C.L.; red (outer), 90\% C.L. The input parameters are shown with a blue star, the best fit with a black dot. . . . . . . . . . . . . 100

6.9 Modified Near Detector neutrino spectrum as used in the Mock Data samples. Plot taken from Marino[66]. Solid line: original true neutrino energy distribution; dashed line: modified distribution. . . . . . . . . . . . . 101

6.10 Contours for mock data, tweaked flux. Light blue (inner), $\Delta \chi^{2}=1.0$; green (middle), 68\% C.L.; red (outer), 90\% C.L. The input parameters are shown with a blue star, the best fit with a black dot. . . . . . . . . . . . . . 102

$6.1168 \%$ CL contours at an exposure of $3.0 \times 10^{20}$ POT for three different input oscillation parameters. The statistical uncertainty on the measurement decreases linearly with $\left|\Delta m_{32}^{2}\right| \ldots \ldots \ldots$. . . . . . . . . . . 103

7.1 Arrival time of selected data events with respect to the expected arrival time of the first neutrino from each relevant NuMI beam spill. The sideband is displayed for $\pm 20 \mu$ s around the beam spill window, demonstrating one cosmic ray muon where 0.4 events are expected. . . . . . . . . . . 109

7.2 Zoomed arrival time of selected data events with respect to the expected arrival time of the first neutrino from each relevant NuMI beam spill. With the $0.25 \mu \mathrm{s}$ binning, the NuMI batch structure is starting to appear at this POT exposure. . . . . . . . . . . . . . . . . . . 110

7.3 Reconstructed track vertex and endpoint locations. Top left: track vertex location in z. Top right: track endpoint location in z. Bottom left: track vertex location in $r^{2}=x^{2}+y^{2}$. Bottom right: track endpoint location

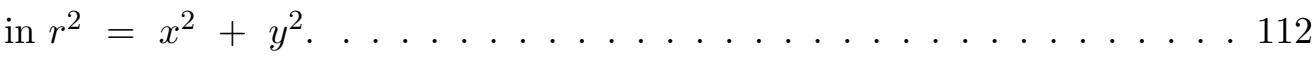

7.4 Reconstructed track vertex locations. Top left: track vertex location in $\mathrm{x}$. Top right: track vertex location in y. Bottom left: track vertex location in u. Bottom right: track vertex location in v. . . . . . . . . . . . . . 113

7.5 Reconstructed track endpoint locations. Top left: track endpoint location in $\mathrm{x}$. Top right: track endpoint location in $\mathrm{y}$. Bottom left: track endpoint location in u. Bottom right: track endpoint location in v. . . . . . . . . . 114

7.6 Various reconstructed event quantities. Top left: quality of track fit. Top right: reconstructed shower energy. Bottom left: cosine of track vertex direction with respect to beam axis. Bottom right: track fit $\chi^{2} / N_{\text {d.o.f. }}$. . 115 
7.7 Reconstructed track momentum from different measurement techniques. Top left: track momentum from range. Top right: track inverse momentum from curvature. Bottom left: track momentum as selected for analysis. Bottom right: track curvature error divided by curvature. . . . . 117

7.8 Three reconstructed variables that determine event PID and the resulting PID distribution. Top left: fraction of event pulse height contributed by track. Top right: track pulse height per plane. Bottom left: number of planes in event. Bottom right: standard PID variable developed by D. Petyt (cut applied below -0.5 to remove NC-like events) . . . . . . . . . . . 118

7.9 Observed minus expected distributions in reconstructed momentum versus angle. An observed deficit presents as a dip below the zero line; an observed excess presents as a peak above zero. . . . . . . . . . . . . . 119

7.10 Reconstructed track momentum. Data: black points. Unoscillated MC: dotted black lines. Best fit MC: solid blue lines. . . . . . . . . . . . . . . . 122

7.11 Reconstructed track direction. Data: black points. Unoscillated MC: dotted black lines. Best fit MC: solid blue lines. . . . . . . . . . . . . . . 123

7.12 Reconstructed track momentum for events used in fit. Data: black points. Unoscillated MC: dotted black lines. Best fit MC: solid blue lines. . . . . 124

7.13 Reconstructed track direction for events used in fit. Data: black points. Unoscillated MC: dotted black lines. Best fit MC: solid blue lines. . . . . 125

7.14 Confidence Level contours for the MINOS fiducial and antifiducial analyses.126

7.15 Confidence Level contours for the MINOS fiducial and antifiducial analyses, Super-Kamiokande, and K2K experiments. . . . . . . . . . . . . 127

7.16 Total protons on target (POT) delivered to the NuMI target during the first two years of operation. The gap represents the 2006 Tevatron shutdown. The total exposure in the standard LE-10 running configuration is $\sim 3.0 \times 10^{20}$ POT. . . . . . . . . . . . . . . . . 127

7.17 Monte Carlo sensitivity 68\% CL contours for the MINOS antifiducial analysis, with two approximate doublings of the Run I data presented here. . 128 


\section{Chapter 1}

\section{Introduction}

The greater our knowledge increases, the greater our ignorance unfolds. -John F. Kennedy

\section{$1.1 \quad$ Neutrino history}

Neutrinos have recently captured the attention of a wider audience than ever before. Entire radio and television programs[6] have been devoted to sharing the known and unknown properties of neutrinos with the public. "What is the neutrino?" even became the question to a Jeopardy answer[7], read by Alex Trebek on network television: "This 8-letter particle named for its lack of charge is being studied by beaming it 450 miles in .0025 seconds." The subject of the MINOS experiment has become a household name, but today's dinnertime discussion has evolved a long way from the uneasy suggestion of the existence of the neutrino by Wolfgang Pauli in his December, 1930 letter to colleagues at a workshop in Tubingen:

Dear Radioactive Ladies and Gentlemen,

As the bearer of these lines, to whom I graciously ask you to listen, will explain to you in more detail, how because of the "wrong" statistics of the $\mathrm{N}$ and ${ }^{6} \mathrm{Li}$ nuclei and the continuous beta spectrum, I have hit upon a desperate remedy to save the "exchange theorem" of statistics and the law of conservation of energy. Namely, the possibility that there could exist in the nuclei electrically neutral particles, that I wish to call neutrons, which have spin $1 / 2$ and obey the exclusion principle and which further differ from light quanta in that they 
do not travel with the velocity of light. The mass of the neutrons should be of the same order of magnitude as the electron mass and in any event not larger than 0.01 proton masses. The continuous beta spectrum would then become understandable by the assumption that in beta decay a neutron is emitted in addition to the electron such that the sum of the energies of the neutron and the electron is constant...

-W. Pauli

Pauli's reluctance to postulate a new particle must be taken in historical context; today, it is commonplace for theorists to invoke new particle fields (for example, to unify gauge coupling constants at high energies that are inaccessible to modern experimentation). However, in the 1930s, the known list of fundamental particles was two: the electron (discovered by Thompson in 1897) and the proton (the positive nucleus discovered experimentally by Rutherford in 1911, and the constituent hydrogen nuclei termed "protons" sometime in the 1920s). Pauli took aim at the beta decay observations of the continuous electron energy spectrum, which appeared to defy the principle of the conservation of energy. By allowing for another light particle in the decay, the total energy of the final state could remain constant. This particle must be electrically neutral to escape detection, prompting the name "neutron".

As fate would have it, the heavy neutral nucleon discovered in 1932 by Sir James Chadwick acquired the name "neutron", leaving Enrico Fermi to propose the name "neutrino" (meaning little neutral one) for the undiscovered particle that Pauli proposed. The physics community would wait over 20 years before the first neutrino was experimentally discovered.

In the meantime, Fermi developed his theory of the weak interaction, which would lay the groundwork for decades of advances in particle physics. The theory predicted the existence of the electron neutrino $\nu_{e}$ as well as its counterpart, the electron antineutrino $\bar{\nu}_{e}$. In what we know today as lepton number conservation, the weak reactions must conserve the electron number $L_{e}$ between the initial and final states. Electrons and electron neutrinos are assigned $L_{e}=+1$; positrons and electron antineutrinos are assigned $L_{e}=-1$. In standard $\beta^{-}$-decay (emission of an electron from an unstable nucleus having $L_{e}=0$ by definition)

$$
n \rightarrow p+e^{-}+\bar{\nu}_{e}
$$

the electron in the final state must be accompanied by an electron antineutrino in order for their lepton numbers to cancel. In a similar manner, during $\beta^{+}$-decay (emission of a 
positron)

$$
p \rightarrow n+e^{+}+\nu_{e}
$$

the positron in the final state must be accompanied by an electron neutrino.

\subsubsection{Discovery of the neutrino}

In Fermi's theory, the neutrino could only interact via the weak nuclear force, roughly $10^{-11}$ times the strength of the familiar electromagnetic force (which the neutrino could not participate in, given its electrical charge of zero). Due to the statistical improbability of detecting a single neutrino, a source was needed that would emit large numbers of neutrinos in a small volume. Early discussions between Fermi and Frederick Reines centered around atomic bomb explosions, but ultimately the controlled fission process in nuclear reactors became the preferred laboratory setting for obvious reasons. In collaboration with Clyde Cowan, Reines built a detector and ran the first experiment in the spring of 1953 near the reactor in Hanford, Washington. Their initial report[8] demonstrated a signal in excess over the expected backgrounds of $0.41 \pm 0.20$ events/minute. This was the first evidence for the antineutrino interaction $\bar{\nu}+p \rightarrow e^{+}+n$. Another detector was soon placed near the Savannah River reactor in South Carolina, and the background signals were better understood by this time. The evidence presented by Reines and Cowan was soon elevated to the status of discovery after publication[9] of their first measurement of the cross section for the antineutrino reaction, with a signal rate of $36 \pm 4$ events/hour. The antineutrino had been discovered, indicating the existence of the neutrino and supporting Fermi's model of weak interactions. Reines later shared the 1995 Nobel Prize in Physics "for the detection of the neutrino".

\subsubsection{The solar neutrino problem}

The earliest experiment to detect the neutrinos being produced in the sun's core of nuclear reactions was performed in the Homestake mine in Lead, South Dakota. Raymond Davis, Jr. led the effort with a group from Brookhaven National Laboratory (BNL), installing a 390,000-liter tank of liquid tetrachloroethylene $\left(\mathrm{C}_{2} \mathrm{Cl}_{4}\right)$ in the old gold mine. The location of 4850 feet underground was optimal for reducing signals from cosmic ray muons and other relevant backgrounds. The experiment searched for the production of ${ }^{37} \mathrm{Ar}$ by the reaction ${ }^{37} \mathrm{Cl}+\nu_{e} \rightarrow{ }^{37} \mathrm{Ar}+\mathrm{e}^{-}$. With a half-life of 35 days, the unstable ${ }^{37} \mathrm{Ar}$ could 
be accumulated on a reasonable timescale and isolated for observation of the radioactive decay.

In 1968, the BNL group reported[10] their experimental method and results, which were at odds with the current predicted neutrino flux from preferred solar models. These results were reported as an upper limit of $0.3 \times 10^{-35} \mathrm{~s}^{-1}$ per ${ }^{37} \mathrm{Cl}$ atom. The expected rate was $2.0 \pm 1.2 \times 10^{-35} \mathrm{~s}^{-1}$ per ${ }^{37} \mathrm{Cl}$ atom. In the same release of Physical Review Letters, John N. Bahcall and Neta A. Bahcall reported[11] revised theoretical predictions that attempted to accommodate the experimental findings, but all credible theories were still predicting a larger solar neutrino flux.

The resulting skirmish in the astrophysics community led many to criticize Davis' experimental methods and to attempt to invalidate his findings. Others believed that our understanding of the solar energy production mechanism was flawed. The position that the observations were done properly and that the theory was also correct did not yet have a champion. Where were the missing electron neutrinos? As time passed, oscillation theory developed as a solution to the problem. The theory was nicely summarized[12] by Hans Bethe (largely responsible for the development of the Standard Solar Model) nearly twenty years after the initial observations. Time would tell, and Davis later went on to share half of the 2002 Nobel Prize in Physics with Masatoshi Koshiba "for pioneering contributions to astrophysics, in particular for the detection of cosmic neutrinos".

\subsubsection{The atmospheric neutrino anomaly}

Another class of neutrinos held mysteries for early experimenters. The reaction chain by which primary cosmic rays interact in the upper atmosphere and produce hadrons (which subsequently decay to muons, electrons, and their associated neutrinos) has a very simple prediction: the ratio of muon-flavor to electron-flavor neutrinos observed at the ground should be 2:1. Most hadron decays produce a $\mu^{+}\left(\right.$or $\left.\mu^{-}\right)$and a $\bar{\nu}_{\mu}\left(\right.$ or $\left.\nu_{\mu}\right)$, depending on the initial electric charge of the hadron. Subsequent decay of the muon by $\mu^{ \pm} \rightarrow e^{ \pm}+\nu_{\mu}\left(\bar{\nu}_{\mu}\right)+\nu_{e}\left(\bar{\nu}_{e}\right)$ results in a total sum of $\mu$-type neutrinos that is twice as large as the sum of $e$-type neutrinos.

These atmospheric (or cosmic-ray) neutrinos cover a wide range of energies, from a few $\mathrm{GeV}$ to several TeV. Experimental methods to detect these neutrinos are different than those required to detect solar neutrinos, which are produced with a much smaller energy ( $\nu_{e}$ produced in the sun's nuclear reactions have energies typical of the binding energy of a nucleus, on the $\mathrm{MeV}$-scale). A typical detector will be built to capture 
the Cerenkov light that is emitted by charged particles passing through water or some suitable transparent target material. Such detectors were traditionally built to search for proton decay, as the sensitivity to detect such a rare event increases with the mass of the detector.

The first large water-based detector was built in the late 1970s in an Ohio salt mine by the Irvine-Michigan-Brookhaven (IMB) collaboration. A larger detector was installed by a Japanese group at Kamioka in the early 1980s. Both experiments observed a lower ratio of muon-type to electron-type neutrinos than was expected by the theory of cosmic ray interactions in the atmosphere, but the anomaly was not specifically discussed until the late 1980s. The Soudan-2 collaboration used a 963 ton steel tracking calorimeter detector, designed in a search for proton decay, to make another measurement of the atmospheric neutrino flavor ratio. Their published result[13] confirmed the previous observations from IMB and Kamioka and came at a time when disappearance theories were gaining momentum as an explanation for both the solar neutrino problem and the atmospheric neutrino anomaly. A thorough review of the anomaly is given in Reference $[14]$.

\subsection{Disappearance theories}

If one accepts the Standard Solar Model and our knowledge of the cosmic ray flux in the upper atmosphere, an explanation is required to account for the "missing" neutrinos. The most attractive explanation, which neatly encompasses missing $\nu_{e}$ from the sun and missing $\nu_{\mu}$ from cosmic rays, is that of neutrino oscillation.

The Standard Model (SM) of particles has been extremely successful in its ability to predict and explain a wide variety of experimental findings, most notably with regard to hadron behavior and the quark sector. Despite SM success in predicting weak interaction physics, the lighter half of the lepton sector has been veiled until quite recently on the time line of particle physics. There was no indication in the SM that neutrinos should have mass, so the assumption stood that neutrinos were massless participants in the weak interactions.

When the quantum mechanical dust settles (see the derivation in Appendix B.1) we find that oscillations can only take place if neutrinos have a rest mass. This simple consequence of the equation has a significant effect on the mass distribution in the universe. Frederick Reines once stated that the neutrino is "the most tiny quantity of 
reality ever imagined by a human being." This little piece of reality, now thought to possess a tiny mass of its own, can add up when one considers that neutrinos are the most abundant particle in the universe. The relic density alone (the big-bang remnant, cooled to below 2 Kelvin) contributes 337 neutrinos and antineutrinos for every cubic centimeter of space[15]. Based on recent cosmological analysis of the Cosmic Microwave Background radiation data[16], the total neutrino mass contribution in the universe is roughly equivalent to that of the normal baryonic matter that makes up the visible stars and galaxies, smaller by a factor of about four. Neutrino mass is now a widely accepted consequence of a combination of high-energy particle physics experiments and astrophysical observations.

Other explanations for neutrino disappearance include decay[17], decoherence[18], and violation of Lorentz Invariance[19]. As stand-alone theories, these tend to be disfavored by most current experimental observations. However, limits on some combination of oscillation and decay (or other combinations of theories) are less stringent, and there remains much work to be done in precisely determining the full theoretical model of neutrino disappearance. New particle scenarios have also been proposed; see Reference $[20]$.

\section{$1.3 \quad$ Experimental tests}

In 1960, Melvin Schwartz published[21] a suggestion to use a proton accelerator to create a neutrino beam. He played a major role in the joint Columbia University-BNL project that would publish[22] the discovery of the muon neutrino in 1962. His paper foreshadowed many of the concepts used in modern long-baseline neutrino experiments. Schwartz shared the 1988 Nobel Prize in Physics with Leon M. Lederman and Jack Steinberger "for the neutrino beam method and the demonstration of the doublet structure of the leptons through the discovery of the muon neutrino".

With theories in place to describe the observations of early neutrino observatories, precision experiments were needed to test the idea that neutrinos could oscillate between flavor eigenstates as they propagated through space. Current experimental evidence tends to favor the oscillation hypothesis, and modern experiments are approaching sensitivity levels that will allow discrimination between the various theories and allow the testing of hybrid theories, such as oscillation plus decay. 


\subsubsection{SNO confirms Standard Solar Model with total NC flux mea- surement}

The Sudbury Neutrino Observatory (SNO) published direct evidence that the electron neutrinos thought to be missing from the solar neutrino flux were oscillating into muon and tau neutrinos. A large underground detector of heavy water was used to detect neutrinos created in the solar ${ }^{8} \mathrm{~B}$ production. By measuring the elastic scattering (ES) reaction $\nu_{x}+e^{-} \rightarrow e^{-}+\nu_{x}$ (sensitive to all neutrino types $x=e, \mu, \tau$, but reduced sensitivity for $\mu$ and $\tau$ ), the neutral current (NC) reaction $\nu_{x}+d \rightarrow p+n+\nu_{x}$ (sensitive to all types $x$ with energy above the $2.2 \mathrm{MeV}$ reaction threshold), and the charged current (CC) reaction $\nu_{e}+d \rightarrow p+p+e^{-}$(sensitive only to $\nu_{e}$, as the solar $B^{8}$ neutrinos fall below the muon and tau energy threshold) over a period from 1999-2001, SNO found a total neutrino flux from the sun that was consistent with the Standard Solar Model prediction. This experiment went beyond the $\nu_{e}$-disappearance measurement to show that the data were consistent with $\nu_{e} \rightarrow \nu_{\mu, \tau}$ oscillations.

\subsubsection{Super-Kamiokande measures atmospheric oscillation parameters}

Expanding on the Kamioka design, Super-Kamiokande, a 22.5 kiloton water Cerenkov detector, began taking data in 1996. The first phase (SK-I) of data was completed in 2001, when an unfortunate accident destroyed roughly two-thirds of the photomultiplier tubes (PMT) used in the detector. Using atmospheric neutrino interactions, SK observed[2] a significant deficit of $\nu_{\mu}$. This led to the first published measurement of the parameters $\left|\Delta m_{32}^{2}\right|$ and $\sin ^{2} 2 \theta_{23}$. See Section 7.6 for a comparison of this and other published measurements of the atmospheric neutrino oscillation parameters.

\subsubsection{K2K measures atmospheric parameters with accelerator neutri- nos}

Utilizing the existing Super-Kamiokande detector, the KEK to Kamioka (K2K) experiment was the first long-baseline $(250 \mathrm{~km})$ accelerator neutrino experiment designed to measure the atmospheric oscillation parameters $\left|\Delta m_{32}^{2}\right|$ and $\sin ^{2} 2 \theta_{23}$ and verify the measurements made previously by SK. With data collected between 1999 and 2004 (after the repairs were made to replace the PMTs lost in the SK accident), K2K observed a significant deficit in the number of $\nu_{\mu}$ interactions coming from the initially pure $\nu_{\mu}$ beam. See Section 7.6 for these results. 


\subsection{Rock muons}

The MINOS Far Detector is located a half mile underground in a retired iron mine, now part of the Soudan State Park in Northern Minnesota. Due to the placement of the detector, it is exposed to a class of events called rock muons. Herein, they will be referred to as rock vertex or RV events. Muons are created in the charged-current interaction of a muon neutrino with an atomic nucleus. When such an interaction occurs within the detector (referred to as contained vertex or CV events), it is possible to measure the hadronic energy (called a shower) that results from the interaction. If the interaction takes place in the rock surrounding the detector cavern, however, the shower is partially or completely absorbed before reaching the detector, depending on the location of the interaction. In many cases, only the muon survives to reach the detector. In the MINOS detectors, the momentum of the muon is measured either by the distance traveled (if the muon stops within the detector volume) or by the curvature of the muon track in the magnetic field (if the muon exits the detector). Determination of the charge of the muon is also made possible by the direction of curvature within the magnetic field.

\subsubsection{Neutrino interactions}

Neutrinos can interact with matter via the weak nuclear force in two main ways, either exchanging a charged $\mathrm{W}^{ \pm}$boson (a charged-current or CC event) or a neutral $\mathrm{Z}^{0}$ boson (a neutral-current or NC event). The details of the final-state particles are similar, with the distinction that a CC event produces a charged lepton $(e, \mu$, or $\tau)$ while a NC event produces a neutral lepton $\left(\nu_{e}, \nu_{\mu}\right.$, or $\left.\nu_{\tau}\right)$. In each interaction, the final state must have the same lepton number as the initial state, meaning that muon neutrinos will either produce a muon neutrino or a muon in the final state.

The total cross section for charged-current muon production has several contributions, shown in Figure 1.1. In the energy region up to $\sim 1 \mathrm{GeV}$, the quasi-elastic (QE) interaction, $\nu_{\mu}+N \rightarrow \mu+N^{\prime}$, dominates. Around $1 \mathrm{GeV}$, the single pion production mechanism, $\nu_{\mu}+N \rightarrow \mu+N^{\prime}+\pi$, reaches its maximal contribution. Both of these cross sections asymptotically approach a constant value, reached by $\sim E_{\nu}=100 \mathrm{GeV}$. The dominant contribution at larger energies comes from deep-inelastic scattering of the neutrino off a quark bound in a target nucleon: $\nu_{\mu}+q \rightarrow \mu+q^{\prime}$. The DIS cross section grows linearly with energy, as seen in Figure 1.1. The quantity plotted is the cross section divided by the neutrino energy. While the $\mathrm{QE}$ and pion production reactions both 
die out beyond $E_{\nu}=10 \mathrm{GeV}$, the DIS contribution reaches a constant differential value around the same energy.

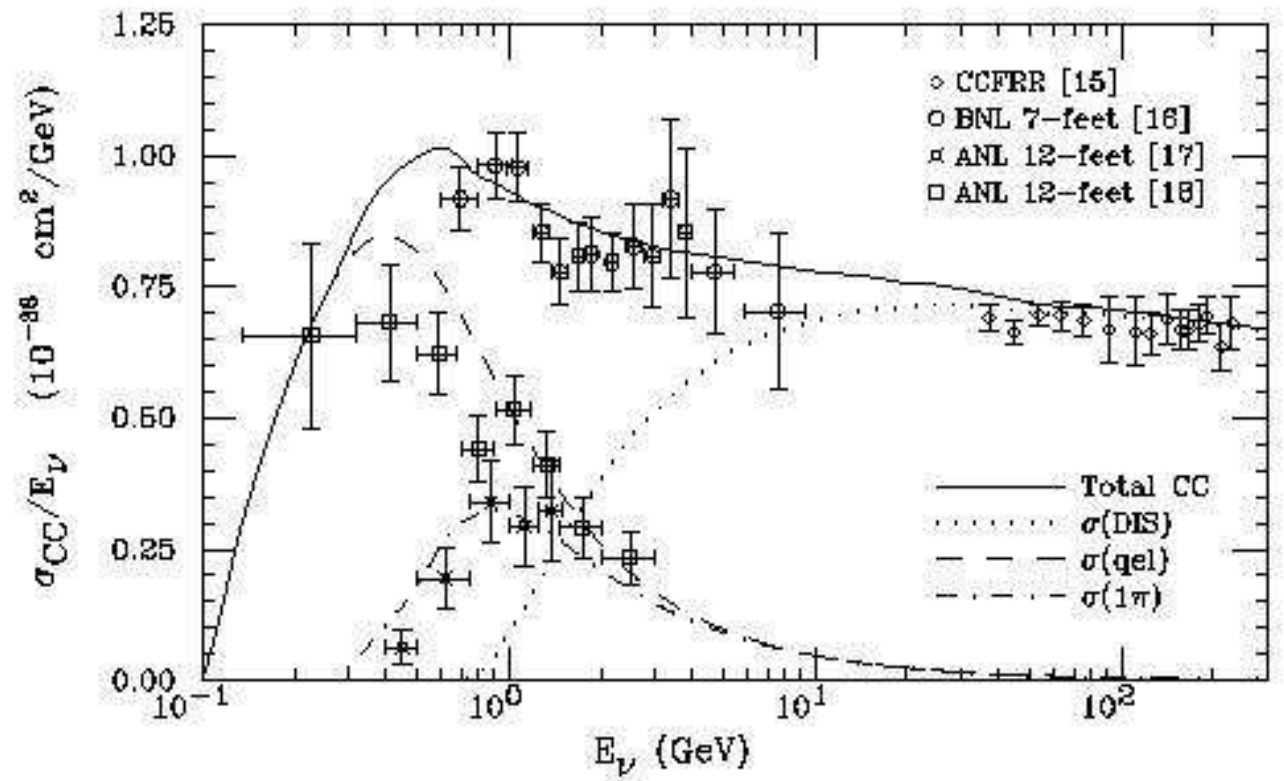

Figure 1.1: Total differential neutrino charged current cross section arising from deep inelastic scattering, quasi-elastic scattering, and pion production. Figure taken from Lipari, Lusignoli, and Sartogo[23].

Neutral current cross sections have been measured, but the uncertainties on these measurements are relatively large compared to the CC cross section measurements. Such measurements are complicated by difficulties in distinguishing charged hadrons (produced in a NC event) such as pions from low-energy muons (produced in a CC event) in detectors.

Beside interactions with the nucleus of an atom, neutrinos can also scatter elastically from electrons in orbit around the nucleus, leaving the initial state electron and neutrino intact in the final state. These interactions are even less common (by a factor of 5000) than $\nu-N$ interactions, with a cross section for $\nu_{\mu}$ on $e^{-}$of $1.6 \times 10^{-42} E_{\nu} \mathrm{cm}^{2} \mathrm{GeV}^{-1}[24]$. 


\subsubsection{Upstream rock}

The presence of a large mass of passive material upstream of the active neutrino detector creates a source of muons which enter the outer surfaces of the detector. These muons are created by CC reactions of muon neutrinos in the passive material. The MINOS Far Detector cavern, located in the historic Soudan Iron Mine, is surrounded by a rock volume that is effectively infinite for the neutrino energies generated by the NuMI beam; there is a uniform flux of rock muons that are created and absorbed in the rock between Fermilab and Soudan.

Consider the rock at the Soudan mine to be an infinite uniform medium upstream of the detector, and take the neutrino cross section to rise linearly with energy. The rate of muons created in the upstream rock that cross a given vertical slice of the detector is then given[25] by:

$$
N_{\mu}^{r o c k}=k^{r o c k}\left(\frac{d \sigma}{d E_{\nu}}, \frac{d E_{\mu}}{d X}\right) \int d E_{\nu} E_{\nu}^{2} n_{\nu}\left(E_{\nu}\right)
$$

where $k^{\text {rock }}$ is a constant which depends on the differential cross section $\frac{d \sigma}{d E_{\nu}}$ (which rises linearly with energy), the muon energy loss rate in the rock $\frac{d E_{\mu}}{d X}$ (which also rises linearly with energy), and the detector geometry; $n_{\nu}\left(E_{\nu}\right)$ is the flux of neutrinos at the detector as a function of energy. Compare this to the rate of muons created inside of the detector that cross the same vertical slice:

$$
N_{\mu}^{\text {det }}=k^{\operatorname{det}}\left(\frac{d \sigma}{d E_{\nu}}\right) \int d E_{\nu} E_{\nu} n_{\nu}\left(E_{\nu}\right)
$$

where $k^{\text {det }}$ is another related constant which depends only on the differential cross section and detector geometry. Notice that the neutrino flux is now sampled by only one power of the neutrino energy. The extra factor of $E_{\nu}$ present in the rock muon rate has the effect of sampling the higher energy regions of the neutrino flux present at the Far Detector. Figure 1.2 displays the ratio of neutrino energy spectra contributing to the Rock Vertex events over the Contained Vertex events, as modeled in Monte Carlo. The linear increase with energy is apparent, demonstrating the greater contribution of high energy neutrinos to the rock muon sample. The plot includes all events as modeled in $\mathrm{MC}$, including those that enter the sides of the detector (not accounted for in Equation 1.3), which modify the ratio slightly from a true linear increase with energy. The greater average energy (roughly $10 \mathrm{GeV}$ ) of neutrino interactions represented in the selected rock muon sample means the events are less sensitive to neutrino oscillations. The standard NuMI beam 
configuration generates the most neutrinos in the region near $3 \mathrm{GeV}$, where the oscillation deficit is expected to be the greatest. Thus, the contained vertex neutrino interactions outside of the fiducial volume provide greater sensitivity to the oscillation parameters than the rock vertex events.

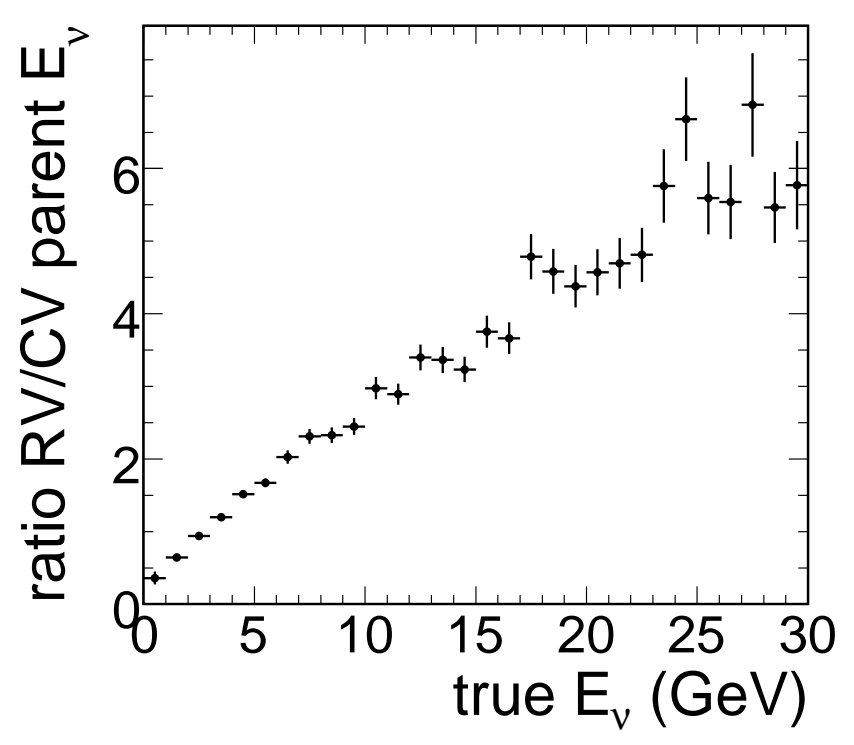

Figure 1.2: Ratio of neutrino energy spectra for rock muon events (RV) over contained vertex events $(\mathrm{CV})$ as modeled in Monte Carlo. The ratio follows the linear increase as expected from the extra power of energy present in the rock muon rate integral (Equation $1.3)$.

The relative number of rock muons and contained-vertex neutrinos is not only dependent on the neutrino energy spectrum, although this is the main determining factor. The geometry of the Far Detector plays a role in determining the relative acceptance. For instance, if the detector had an extremely large head-on cross sectional area and was shorter along the beam direction, the number of rock muons would overwhelm the number of contained-vertex neutrino events due to the much larger "effective mass" of rock for detecting muons over the detector mass sensitive to neutrino interactions. Another example is if the MINOS Far Detector had been upgraded to contain three super-modules (from the two it has today). The acceptance for rock muons entering the 
sides of the detector would cause this event sample to increase by $50 \%$. Unlike the last example, the number of contained-vertex events would also increase by $50 \%$ in this case. The determining factor in relative sensitivity is the ratio of detector surface area to its volume.

\subsubsection{Other experimental observations of rock muons}

The aptly-named Large Volume Detector (LVD) has been placed underneath the Gran Sasso mountain in central Italy with the purpose of detecting neutrinos from gravitationally collapsing stars, and has been in operation since 1991. With the turn-on of the CERN Neutrinos to Gran Sasso (CNGS) beam, LVD is now serving a beam-monitoring purpose. LVD detects $\sim 120$ muons each day during nominal beam running, resulting from neutrinos interacting with the rock surrounding the detector. The detector[26] measures roughly $13 \mathrm{~m} \times 11 \mathrm{~m} \times 25 \mathrm{~m}$, with a total mass around 2 kilotons.

Because the current purpose of CNGS is to establish the appearance of $\nu_{\tau}$ in a $\nu_{\mu}$ beam (to be observed by the OPERA[27] experiment), the peak of the neutrino energy distribution must be at higher energy in order to provide enough events above the tau production threshold. $400 \mathrm{GeV}$ protons are extracted from the CERN Super Proton Synchrotron (SPS) to provide an average neutrino energy of $\sim 17 \mathrm{GeV}$. Oscillation probabilities averaged over the CNGS neutrino energy spectrum are quite low due to the higher energies involved, such that only 10-15 tau events are expected over the course of the experiment. Due to this fact, the use of rock muons in the CNGS beam to make a measurement of the parameters $\left|\Delta m_{32}^{2}\right|$ and $\sin ^{2} 2 \theta_{23}$ is not feasible. The observed muon rate at LVD is in agreement[28] with the expected rate obtained with a similar Monte Carlo model as that used in this dissertation.

Another experiment located in the Gran Sasso facility, Borexino, has also confirmed rock muon observation for CNGS beam-induced events. The Borexino detector is a liquid-scintillator sphere, surrounded by veto spheres containing ultra-pure water. During initial filling of the outer instrumented region with water, an active mass was present for detecting Cerenkov light from passing muons. In this nascent state, the Borexino detector observed[29] twelve through-going muons with an expectation of ten during the CNGS beam exposure. The events have timing signatures consistent with the CNGS beam spill times, allowing discrimination against the cosmic ray background. During full CNGS operations, Borexino and LVD will both act as muon monitors in conjunction with the OPERA search for tau appearance in the muon neutrino beam. 


\section{Chapter 2}

\section{The MINOS experiment}

What we observe is not nature itself, but nature exposed to our method of questioning.

-Werner Heisenberg

One of the earliest discussions of neutrino oscillations is found in a 1969 letter by Gribov and Pontecorvo[30], in which the authors speculate about the consequence of lepton nonconservation:

In previous publications[31][32] there was shown that lepton nonconservation leads to the possibility of oscillations in vacuum between various neutrino states, and, generally speaking, acts in the sense of decreasing the number of detectable solar neutrinos with respect to the number expected theoretically under the assumption that lepton charges are strictly conserved.

While Pontecorvo presented his oscillation theory as a solution to the solar neutrino problem, extensions of the theory would eventually arise to explain the entire range of neutrino data, including the atmospheric neutrino observations.

The first suggestion that neutrino oscillations could be probed with a long baseline experiment was made in 1977 by Mann and Primakoff[33]. In a clear illustration, the authors show how a neutrino beam could be aimed down into the earth to resurface at a distance of approximately 1,000 km. Mann and Primakoff suggested that the existing neutrino beam at Fermilab be aimed at a suitably massive detector in the Quebec Province of Canada, after sending the beam through a smaller detector placed near the 
source. Both detectors would need the capability to detect and distinguish between electron and muon charged current (CC) events. The ratio of observed to expected muon events at the second detector was given as the most natural test for oscillations, having an accuracy limited by the uncertainty of the rate measurement in the first detector. The authors recognized the implications of observed neutrino oscillations at this stage: "(i) The mass of at least one of the neutrino types would be nonzero, (ii) the separate conservation of muon lepton number and electron lepton number would not hold, and (iii) the total number of neutrino types would be determined."

The late 1980s fostered the planning stages of such an experiment. Due to the belief that $\left|\Delta m_{32}^{2}\right|$ was larger than we now understand it to be, such planning focused on a neutrino beam with a relatively large average energy. One consequence of the higher neutrino energies is the dominance of the beam event rates by rock muons (see Equations 1.3 and 1.4). As a result, one of the main oscillation measurements was planned as an observation of the ratio of rock muons to contained vertex neutrino events. For the planned beam and detector parameters, this ratio decreases from $R_{\mu / \nu}=3.51$ (with no $\nu_{\mu}-\nu_{\tau}$ mixing) to $R_{\mu / \nu}=2.86$ (near maximal mixing) with a statistical uncertainty of 0.06[34]. As Super-Kamiokande data on the atmospheric mixing parameters was made available, it became clear that a lower beam energy was required, given the $735 \mathrm{~km}$ baseline, in order to place the peak of the energy distribution at the optimal $L / E$ position to observe the most significant deficit in the event rate at the Far Detector. This reduced the expected rock muon rate, such that the ratio to contained neutrino events was expected to be approximately 1:1.

\subsection{Historical development}

Even as the Fermilab Main Injector was in its proposal stage, physicists from around the world jumped at the possibility of an intense neutrino source that could be aimed at their far-flung detectors. Three main groups interested in long-baseline oscillation experiments responded to the call for submissions at the 1989 Fermilab Workshop on Physics at the Main Injector. The Deep Underwater Muon and Neutrino Detection (DUMAND) project, which involved a $6000 \mathrm{~km}$ baseline to Hawaii, was ultimately abandoned after a failed test early in the construction phase. Soudan 2, which involved a $730 \mathrm{~km}$ baseline to Minnesota, had established a detector deep underground and was already collecting data. The Irving Michigan Brookhaven (IMB) experiment, which involved a $580 \mathrm{~km}$ 
baseline to Ohio, had established a water Cerenkov detector underground. Each of these submissions included estimates of rock muon rates and tests for oscillation searches using measured deficits in the muon rate.

Eventually, two proposals for long-baseline experiments were submitted to Fermilab in 1990: P-805 for the IMB detector, and P-822 for the Soudan 2 detector. Due to an accident that resulted in the collapse of a large section of the wall of the IMB underground laboratory, P-805 was withdrawn. The Fermilab Physics Advisory Committee (PAC) was optimistic about P-822 but suggested[35] that a detector much larger than Soudan 2 would be needed.

The Main Injector Neutrino Oscillation Search (MINOS) collaboration was born in late 1994, consolidating the interested parties into one long-baseline experimental effort at Fermilab. In the same period, there was another proposal coming through Brookhaven National Lab (BNL) for a competing long-baseline experiment that would use the AGS accelerator. The Department of Energy could only afford to fund one of the proposals. Consequently, a Sub-panel of the High Energy Physics Advisory Panel (HEPAP) was formed to advise the decision between the two proposals. After meeting at both Fermilab and BNL, and carefully examining the physics capability and estimated costs of each experiment, the Sub-panel recommended that MINOS be chosen over the BNL proposal. Its report stated that "The discovery of neutrino oscillations, and consequently the discovery of neutrino mass, would constitute a major breakthrough in particle physics and the first evidence of physics beyond the minimal Standard Model." HEPAP gave full support to the Sub-panel's decision, thus MINOS had its financial support and a mandate to probe the properties of this elusive half of the lepton sector.

\subsection{Description of goals in context of rock muons}

Two main goals were set up[35] at the outset of the experiment, addressing the possibility of neutrino oscillations. If neutrinos do not oscillate in the region of parameter space accessible to MINOS, the largest possible area of parameter space should be excluded. If oscillations do occur in this region, MINOS was to demonstrate their existence, precisely measure the relevant parameters, and specify which oscillation modes are responsible for the observed effects.

The desire to be able to identify $\tau$ appearance was strong at this point. The expected running configuration of the neutrino beam was at a higher average energy than our 
current configuration because there was evidence from Kamiokande[36] measurements of atmospheric neutrino events that the mass squared difference parameter $\Delta m_{32}^{2}$ was in the range $10^{-2}$ to $10^{-1} \mathrm{eV}^{2} / \mathrm{c}^{4}$. Given the fixed baseline distance of $735 \mathrm{~km}$, this dictated the peak of the neutrino spectrum to be fixed around $10 \mathrm{GeV}$ in order to maximize the oscillation probability $P\left(\nu_{\mu} \rightarrow \nu_{\tau}\right)$ for the greatest number of events. At these planned beam energies, the number of rock muons compared to contained vertex neutrino interactions was a factor of two or three higher, depending on the detector model. Due to this muon abundance, many early analysis simulations focused on using the rock muon rate as a test for oscillations, either as a stand-alone disappearance measurement or in a comparison to the contained-vertex neutrino event rate.

A GEANT3-based Monte Carlo study was carried out by Hans-Jochen Trost[37] in 1995 in which a detector resembling the reference MINOS Far Detector design at the time (using $\sim 12 \mathrm{kT}$ of steel, concrete, and active Iarocci tubes) was enclosed in $340 \mathrm{kT}$ of rock, modeled as $50 \mathrm{~m}$ deep upstream and $10 \mathrm{~m}$ deep on all sides of the detector hall.. Trost concluded that the detector as modeled ( $62.4 \mathrm{~m}$ long, $8 \times 8 \mathrm{~m}$ face) was sensitive to a region of at least $130 \mathrm{kT}$ of rock, which extended beyond $50 \mathrm{~m}$ deep upstream and about $4.5 \mathrm{~m}$ deep into the side volume. The detector hall was modeled as $72 \mathrm{~m}$ long, $14 \mathrm{~m}$ wide, and $12 \mathrm{~m}$ high, leaving $3 \mathrm{~m}$ air gaps on the sides of the detector, a $1.5 \mathrm{~m}$ gap on the bottom, and a $2.5 \mathrm{~m}$ gap on the top. For comparison with the author's rock model (see Section 3.2.3), this is equivalent to a sensitive target region $7.5 \mathrm{~m}$ transverse to the sides of the detector. With a simulated exposure of $2 \mathrm{kT}$-years using the high-energy beam and this detector model, Trost was able to set an sensitivity limit of $\left|\Delta m_{32}^{2}\right|<1 \times 10^{-2} \mathrm{eV}^{2}$ $/ \mathrm{c}^{4}$, near the region where the parameter was expected to lie.

Without a precise measurement of $\left|\Delta m_{32}^{2}\right|$ to guide fixed locations of the neutrino beam components, it was desirable to construct a beam line that would allow flexibility to search a wide region of oscillation parameter space. This was ultimately achieved by the NuMI project, which used movable electromagnetic horns and a movable carbon target to allow focusing of different energy regions of the hadron spectrum produced from the proton collisions on target.

\subsection{The NuMI beam}

The Neutrinos from the Main Injector (NuMI) project extracts $120 \mathrm{GeV}$ protons from the MI at Fermilab and directs them at a graphite target in the beam line. This is referred 
to as one beam spill, which occurs with a nominal cycle time of $2.2 \mathrm{~s}$. One beam spill contains $\sim 2 \times 10^{13}$ protons; integrated over the run period, this is the NuMI exposure reported throughout this document as Protons on Target, or POT. Figure 2.1 depicts the beam line. The resulting hadrons (mainly charged pions and kaons) emerging from the nucleon collisions in the target are focused by two electromagnetic horns. These focused hadrons enter the decay pipe roughly $40 \mathrm{~m}$ from their creation point in the target. The hadrons have an energy-dependent spot size after being focused by Horns 1 and 2; the relative orientations of and size of current running through the horns dictates the energy range of hadrons most focused in this process. The decay pipe is evacuated and allows for a majority of hadrons to decay before reaching the hadron monitor, approximately $700 \mathrm{~m}$ from the target. A section of dense absorber permits only the decay products (muons and neutrinos) to proceed. Three muon monitors are placed in separate alcoves in the downstream rock to allow further beam profile monitoring. The muons then encounter $\sim 300 \mathrm{~m}$ of rock and range out before reaching the MINOS Near Detector. At this point, a mostly pure beam of muon neutrinos is all that remains, and the vast majority of neutrinos pass through the Near Detector without a trace. We observe on the order of ten neutrino interactions per spill in the ND, depending on the initial proton intensity.

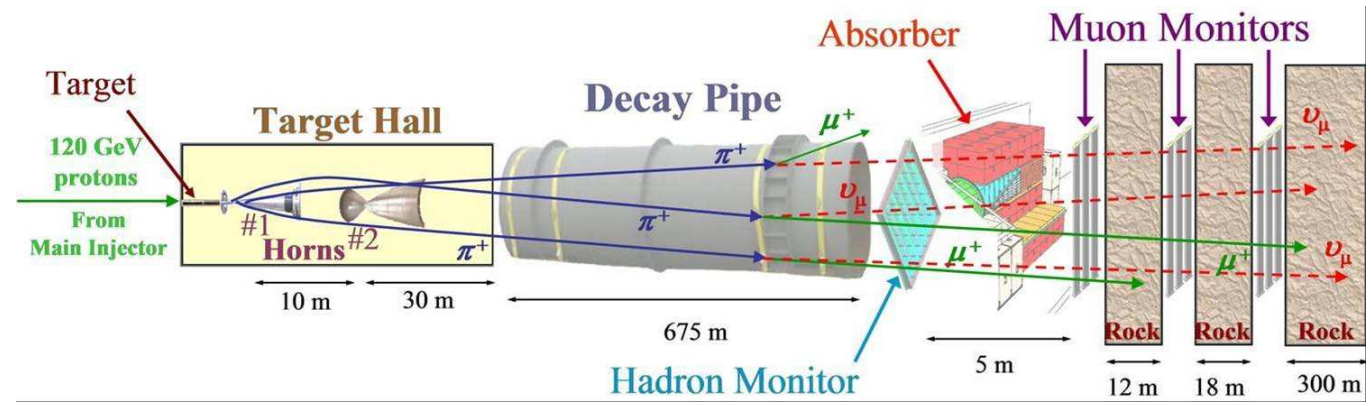

Figure 2.1: Cartoon diagram of the NuMI beam line at Fermilab (not to scale).

Neutrinos in the NuMI beam travel from Fermilab to Soudan, Minnesota, the location of the MINOS Far Detector. The journey takes about $2.5 \mathrm{~ms}$. See Figure 2.2 for a map of this trip. Google Maps ${ }^{\mathrm{TM}}$ suggests a circuitous path involving I-90, I-94, US-53, and MN-169, but the neutrinos prefer the more direct route beneath the surface. Essentially all of the neutrinos pass undetected through the Far Detector and leave the earth's surface about $12 \mathrm{~km}$ downstream, causing a strobe-like $\nu_{\mu}$ beam to penetrate deep into 
space, blinking about every two seconds and spinning with the earth's rotation. MINOS detects about three to six beam events in the Far Detector each day during normal beam operation.

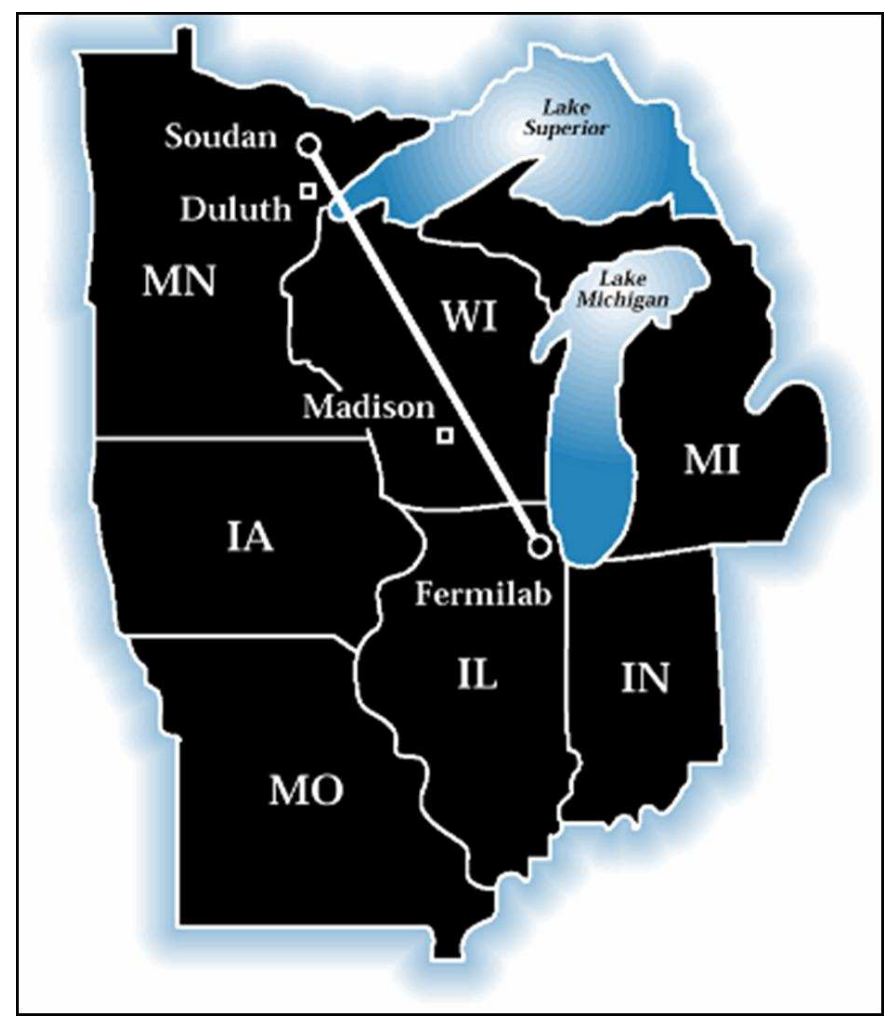

Figure 2.2: Map showing the trip taken by NuMI neutrinos from Fermilab to Soudan.

To adjust for the earth's curvature, the NuMI beam line must point down at Fermilab by $58 \mathrm{mrad}\left(\sim 3^{\circ}\right)$ relative to the local horizontal. The two MINOS detectors are separated by $735 \mathrm{~km}$ of rock. At the midway point, the beam passes $10 \mathrm{~km}$ beneath the surface of Wisconsin. When the beam arrives at the Soudan Mine $\sim 700 \mathrm{~m}$ below ground level, it is pointing $\sim 3^{\circ}$ up relative to the local horizontal. Because of the decay kinematics of the hadrons in the NuMI decay pipe, the $\nu_{\mu}$ in the beam are not perfectly collimated, and the beam diverges as it travels due to the slight transverse momentum carried by some neutrinos. By the time it reaches the Far Detector, the central beam spot (which is characterized by a Gaussian shape in the transverse plane) is approximately 
$1 \mathrm{~km}$ wide.

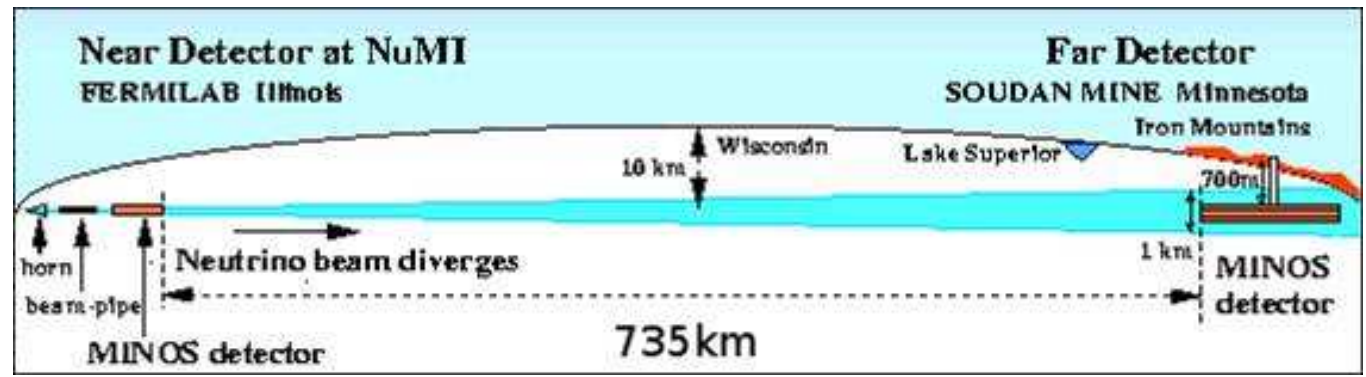

Figure 2.3: Profile of the NuMI beam as it passes beneath Illinois, Wisconsin, and Minnesota. The central beam spot size is roughly $1 \mathrm{~km}$ wide at the Far Detector.

The NuMI target[38] is made of graphite, with a density of $1.78 \mathrm{~g} \mathrm{~cm}^{-3}$. The main target uses 47 vertical segments, each of which is $20 \mathrm{~mm}$ long and $6.4 \mathrm{~mm}$ wide, separated by $0.3 \mathrm{~mm}$ of space between segments. The total target length is $95.38 \mathrm{~cm}$. The height of the segments transverse to the beam is between 15 and $18 \mathrm{~mm}$, depending on the longitudinal position. Water cooling pipes run along the top and bottom edges of the target, connected at the end by a round pipe segment that allows flow from top to bottom without obstructing the initial proton beam.

After the MINOS group was well-established, Super-Kamiokande released evidence for the atmospheric neutrino oscillation parameters that gave a significantly lower measurement of $\left|\Delta m_{32}^{2}\right|$ than had previously been indicated by Kamiokande's atmospheric sample, falling in the range $1.5-3.5 \times 10^{-3} \mathrm{eV}^{2} / \mathrm{c}^{4}$. It became clear that a lower peak energy would be necessary to make the best observation of the oscillation minimum. The NuMI target and horns were designed with such flexibility in mind. By moving the relative longitudinal positions of the target and horns and changing the magnitude of the horn current, different beam profiles can be attained. Figure 2.4 demonstrates the different beam configurations and the resulting reconstructed neutrino energy spectra in the Near Detector. The panel in the upper right, labeled "LE-10/185kA", shows the configuration selected for the disappearance analysis. "LE-10" marks the low-energy configuration, in which the target is positioned $10 \mathrm{~cm}$ upstream of Horn 1; "pME" and "pHE" mark the medium- and high-energy configurations, in which the target is positioned further upstream. The standard horn current (both horns are in series and share the same current) is $185 \mathrm{kA}$, but this can be adjusted as well to further fine-tune the 
shape of the neutrino spectrum. The various other beam configurations are mainly used to "tune" the GNuMI Monte Carlo spectrum using a parameterization in transverse and longitudinal momentum of the parent hadrons decaying in the beam line (see Section $5.2)$.

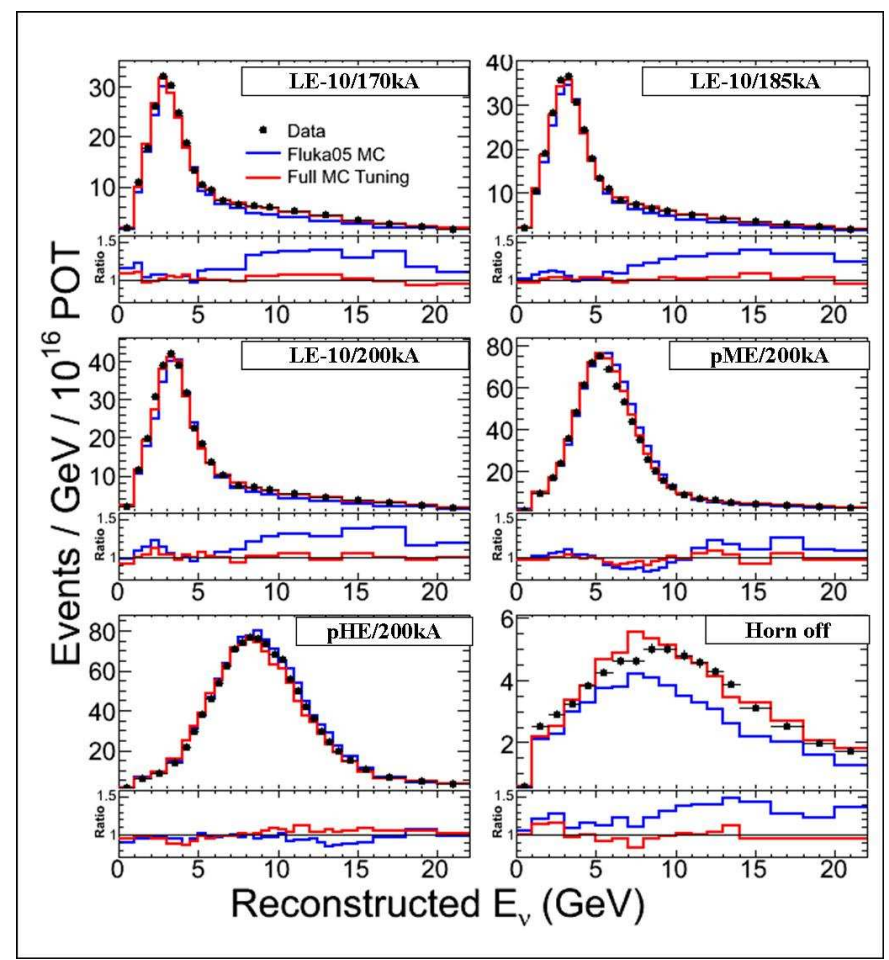

Figure 2.4: Various NuMI beam configurations and their corresponding reconstructed neutrino energy spectra as observed with the MINOS Near Detector. Data is shown with black points, the original Monte Carlo in blue, and the tuned MC in red. LE-10 indicates the standard low-energy target position (retracted $10 \mathrm{~cm}$ from the edge of Horn 1). Standard horn current is $185 \mathrm{kA}$. The pME target location is retracted $1.0 \mathrm{~m}$ from the edge of Horn 1, and the pHE target location is retracted $2.5 \mathrm{~m}$.

The delivery of protons from the Main Injector has three modes, determining the bunch structure of neutrinos emerging from the target. Other experiments at Fermilab require protons from the Main Injector, which is the main force driving the different operating modes. Two of the modes can be included in the label "five-batch spills" and the other mode is labeled "six-batch spills". Both spill types deliver the entire proton load 
within a $10 \mu$ s window. Figure 2.5 displays the five- and six-batch structures within this window. The six-batch mode is known as "NuMI-only" and delivers the most protons on target per spill, distributed evenly over batches 1-6. The two five-batch modes are known together as "mixed" mode, which are limited to either batches 1-5 or 2-6, depending on the configuration. This results in the first and sixth batches accumulating fewer total events.
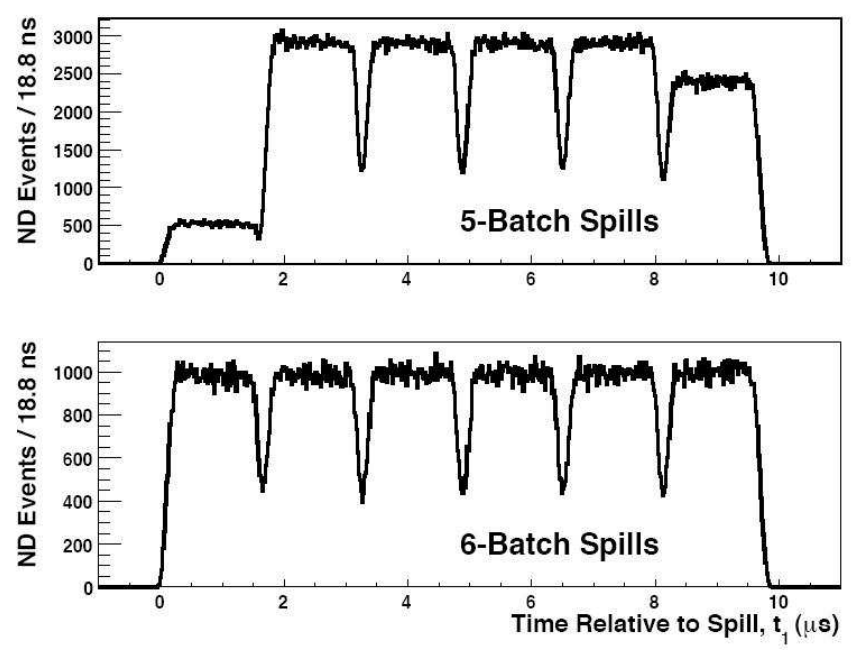

Figure 2.5: Neutrino event arrival times at the Near Detector. Figure taken from $\operatorname{Tagg}[39]$.

The beam is monitored at several locations along the NuMI line. Among the most important monitors are the hadron and muon monitors shown in Figure 2.1 and discussed earlier in this section. Software is in place in the MINOS Control Room at Fermilab that allows shift workers to view the performance of the beam via these hardware monitors in real time and alert appropriate experts in the accelerator Main Control Room if anomalies are present. The intensity profile transverse to the beam axis is one important facet of operation that must be scrutinized, as the beam spot size and position relative to the target origin can affect the neutrino spectrum downstream. A combination of automated hardware and software devices along with vigilant shifters have kept the beam performing consistently over the past two years. The first neutrinos from NuMI were recorded in the MINOS Near Detector during commissioning runs in January 2005, and the first event at the Far Detector was recorded in March 2005. Since mid-May 2005, the majority of run 
time has been spent in the LE-10 position, with several million ND events and $\mathcal{O}(1000)$ FD events recorded in this configuration, used for the $\nu_{\mu}$ disappearance analysis.

\subsection{The MINOS detectors}

Due to the desire to eliminate sources of uncertainty between the Near and Far Detectors, they were constructed as nearly identical magnetized steel calorimeters, with the main differences being the size and electronics layout. The Calibration Detector was also constructed in a similar manner to the ND and FD to allow for absolute calibration of the detector performance using a test beam at CERN.

Common aspects of the two detectors include the plane thickness $(6 \mathrm{~cm})$ and orientations. Each instrumented plane is composed of a steel layer, a scintillator layer, and an air gap. The thickness of each steel plane is $2.54 \mathrm{~cm} \pm 0.76 \mathrm{~mm}$. A $1 \mathrm{~cm}$ thick scintillator plane lies downstream, adjacent to the steel plane. The remaining gap to the next steel plane is left open for structural reasons, allowing mechanical spacers to be placed that accurately set the plane-to-plane distance to $2.4 \mathrm{~cm}$.

The scintillator planes are composed of $4.1 \mathrm{~cm}$ wide polystyrene strips. The polystyrene base is doped with fluors (PPO and POPOP) and co-extruded with a titanium dioxidedoped plastic outer layer, designed to reflect light created by the passage of charged particles back into the strip. When a particle traverses a strip, ionized molecules in the plastic excite a chain of fluor absorption and re-emission, shifting the light from the UV range down into the blue region of the spectrum. A small groove runs down the center of each plastic strip in which a Kuraray Y11 wavelength-shifting optical fiber is placed. The fiber, $1.2 \mathrm{~mm}$ in diameter, collects blue light created in the strip, down-converts the wavelength into the green region (where transmission is optimal in the fiber) and transmits it to each end of the strip. At this point, a manifold at each module edge passes the light into clear fibers and transmits it to multi-anode photomultiplier tubes (PMTs). Each tube multiplies the charge deposited by photon excitation; this charge is then integrated and read out by front-end electronics chips. Two calibration systems are present at the front end to track detector response over time: a light calibration and a charge calibration. The Light Injection (LI) system flashes LED lamps with known ultraviolet light output into modules so that the time dependence of the detector response to light can and individual channel gains can be monitored precisely over time. The charge injection system is similar, but bypasses the PMT, injecting known amounts of 
charge into the front-end electronics to monitor their response. The LI system activity is tagged during normal beam operation to allow removal of light injection "events" present in the spill data stream. See Section 4.2.1 for a description of the cuts used to remove LI activity.

Alternating scintillator planes are oriented at $\pm 45^{\circ}$ to the vertical y-axis. These planes are labeled $u$ and $v$; when facing the upstream face of each detector, the v-plane has its strips rotated $45^{\circ}$ counterclockwise from the y-axis, while the u-plane has its strips rotated $45^{\circ}$ clockwise. These two views allow a stereo image to be formed when a charged particle track lights up a series of strips along the length of the detector.

Both the Near and Far Detectors are monitored and controlled in the MINOS Control Room at Fermilab; temporary control can be handed over to local crews in the respective detector halls. Ambient temperature, humidity, coil cooling water, electronics noise rates, calibration, and several other quantities are monitored in real time by the shifter in the control room. Automated alarms and attentive shifters catch problems early and can alert local crews to take appropriate action. This monitoring has kept the Far Detector live time well over $99 \%$ during NuMI beam operation.

\subsubsection{Calibration Detector}

The Calibration Detector (CalDet) was constructed using square planes, with 24 strips oriented vertically (in the v-planes) and horizontally (in the u-planes). The detector used the same plane construction as would be used in the ND and FD, and consisted of 60 planes along the beam axis. Figure 2.6 shows the detector in place at CERN. The dimensions of the detector were $1 \times 1 \times 3.6 \mathrm{~m}$ and its mass was $12 \mathrm{~T}$. CalDet was placed in the T7 and T11 test beams along the PS ring at CERN in order to characterize the detector response to particles of known energy. Both ND and FD electronics were used to read out the CalDet planes in order to characterize any differences.

Detector response to protons, pions, electrons, and muons was studied in the test beams at CERN. The particles ranged in energy from $200 \mathrm{MeV}$ to $10 \mathrm{GeV}$. Details of the Calibration Detector and shower energy resolution (relevant to the fiducial neutrino analysis) can be found in References [40] and [41]. 


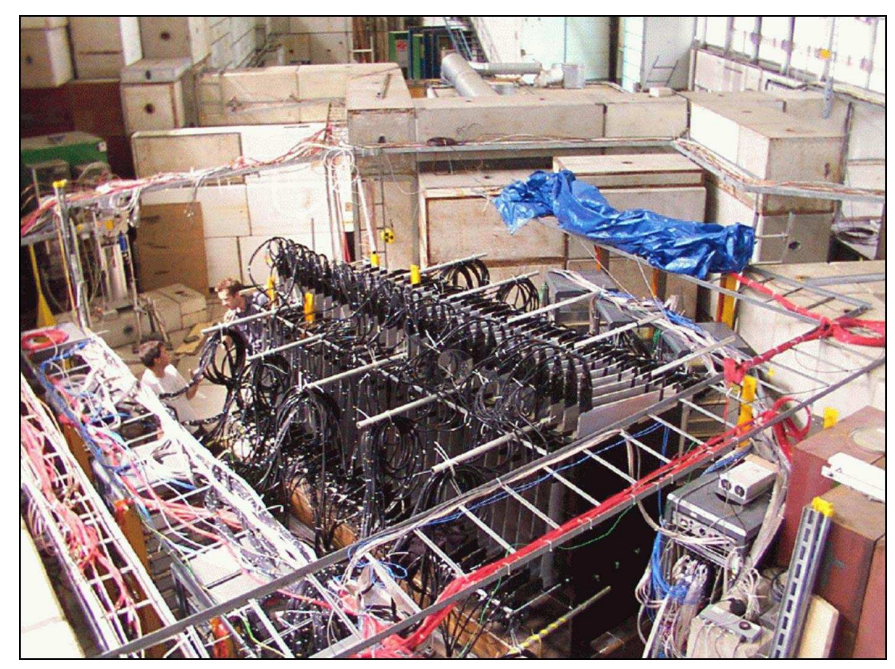

Figure 2.6: Overhead view of the MINOS Calibration Detector in place at CERN. The detector is now decommissioned.

\subsubsection{Near Detector}

The Near Detector lies $\sim 1 \mathrm{~km}$ from the NuMI target and $90 \mathrm{~m}$ under the surface of Fermilab. The main purpose of the ND is to measure the neutrino energy spectrum before traveling far enough for the effects of oscillation to manifest as an energy-dependent deficit. The total ND mass is 980 tons. It contains 282 planes, 153 of which are instrumented with active scintillator. The detector has an asymmetric octagonal cross section, $3.8 \mathrm{~m}$ high and $4.8 \mathrm{~m}$ wide. The length of the detector is $16.6 \mathrm{~m}$. The fiducial volume for neutrino interactions (discussed in Section 4.1) begins $1.0 \mathrm{~m}$ deep into the front face and ends $4.0 \mathrm{~m}$ downstream; the radius of this volume is $1.0 \mathrm{~m}$, centered on the beam spot $50 \mathrm{~cm}$ to the left of the central detector axis.

A coil hole passes through the right-center of each plane to allow the high-current (40 kA-turns) electromagnetic coil to run the entire length of the detector. The beam spot is located left of center, $1 \mathrm{~m}$ away from the coil hole. The electromagnetic coil generates a magnetic field that varies from 1 - $2 \mathrm{~T}$ throughout the detector.

The upstream 120 planes (the calorimeter) are all instrumented with active scintillator; every fifth plane is covered over most of the area (96 scintillator strips wide), while 
the rest are covered only in the portion of the plane to the left of the coil hole (64 scintillator strips wide), centered on the beam spot. This provides detailed information on the neutrino interactions inside the fiducial volume. The downstream 162 planes (the spectrometer) have every fifth plane instrumented with scintillator that covers the entire plane. The spectrometer tracks muons that are created in charged-current interactions upstream. By measuring the amount of curvature of the muons in the ND magnetic field, a determination of the momentum can be made. See Section 5.1 for a description of the ND performance in measuring the momentum of muons. A full discussion of the muon calibration and Near Detector electronics can by found in Reference [42].

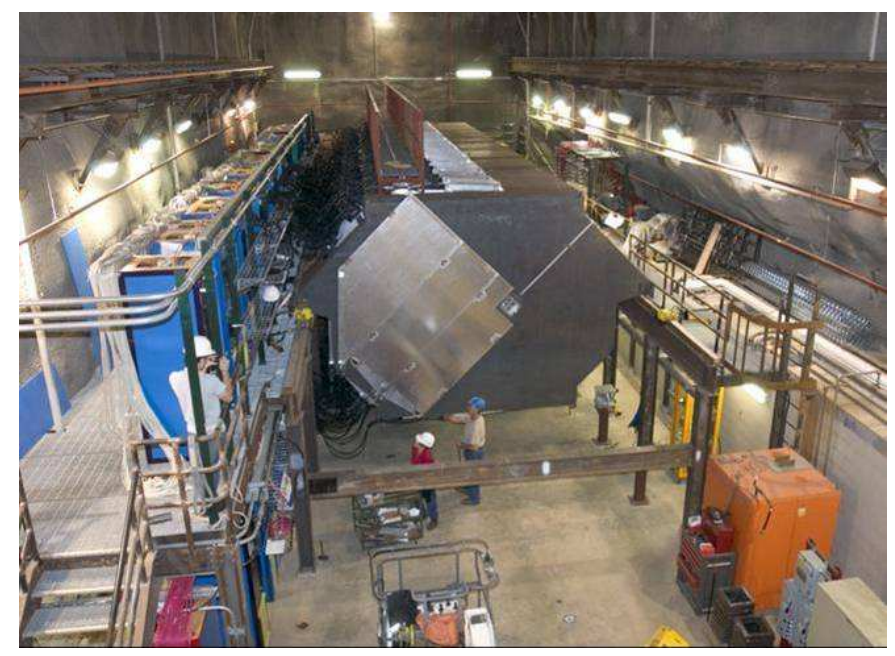

Figure 2.7: Upstream view of the MINOS Near Detector. Note the human subjects for sense of scale.

\subsubsection{Far Detector}

The Far Detector is positioned $735 \mathrm{~km}$ from Fermilab and $700 \mathrm{~m}$ under the surface of the Soudan Mine State Park. The main goal of the Far Detector is to characterize the neutrino energy spectrum at a large distance from the source, where oscillation effects can be observed. It contains 486 planes and has a total mass of $5.4 \mathrm{kT}$. The detector is separated into two SuperModules, each about $15 \mathrm{~m}$ long and separated by a $1.1 \mathrm{~m}$ gap (the "SM gap"). SM 1 contains 250 planes (numbered 0-249) and SM 2 contains 236 planes (numbered 250-485). The first upstream plane of each SuperModule is not 
instrumented with scintillator and acts as passive target. Each SuperModule has a separate electromagnetic coil running through the center coil hole, passing $15 \mathrm{kA}$-turns and generating an average field of $\sim 1.5 \mathrm{~T}$ inside the steel planes of the detector. The SM gap allows enough room for the passage of the separate return coils of both SM 1 and SM 2. The scintillator portion of each plane is assembled of eight modules of differing shape and strip content, depending on the module transverse location in the plane. The cross section transverse to the beam direction is octagonal with an $8 \mathrm{~m}$ width/height. Each plane is fully instrumented with 192 scintillator strips across the face.

Both ends of strips are read out to Hamamatsu M16 PMTs. Each PMT contains 16 pixels, each of which read out eight multiplexed fiber ends. The strips corresponding to each of the eight fibers terminating at the same pixel are physically separated by $\sim 1 \mathrm{~m}$ within the plane, to provide the best separation possible when the signal must be assigned to a strip. To unravel the possible combination of hit patterns when one pixel is activated, a different multiplexing scheme is used on each end of the detector planes. Resulting errors in assigning strip activity to each activated PMT pixel are typically caught at the reconstruction stage. Crosstalk between PMT pixels is also possible, but typically results in distinct hit patterns that can be filtered out in the offline event reconstruction stage. Module manifolds are routed into MUX boxes, each containing three PMTs. Each PMT pixel is read out by a Viking VA chip, grouped onto Front-end Boards. The analog signal is passed from the Front-end Board to a VARC Mezzanine Module (VMM) for digitization of ADC counts. The timing of hits is governed by GPS timestamps that can be synchronized with NuMI spill times to look for coincidence with the beam gate. A full description of the Far Detector readout electronics can be found in Reference [43].

The top surface and sides of the detector are covered by two layers of active scintillator modules, which serve as a veto shield for atmospheric muons that can enter a gap between planes and start depositing energy within the fiducial volume of the detector. In the antifiducial muon analysis presented here, the information from the veto shields is not used, as the track arrival time and transverse momentum are sufficient to mitigate the background from cosmic ray muons; see Section 4.5.1 for a discussion of this background removal. 


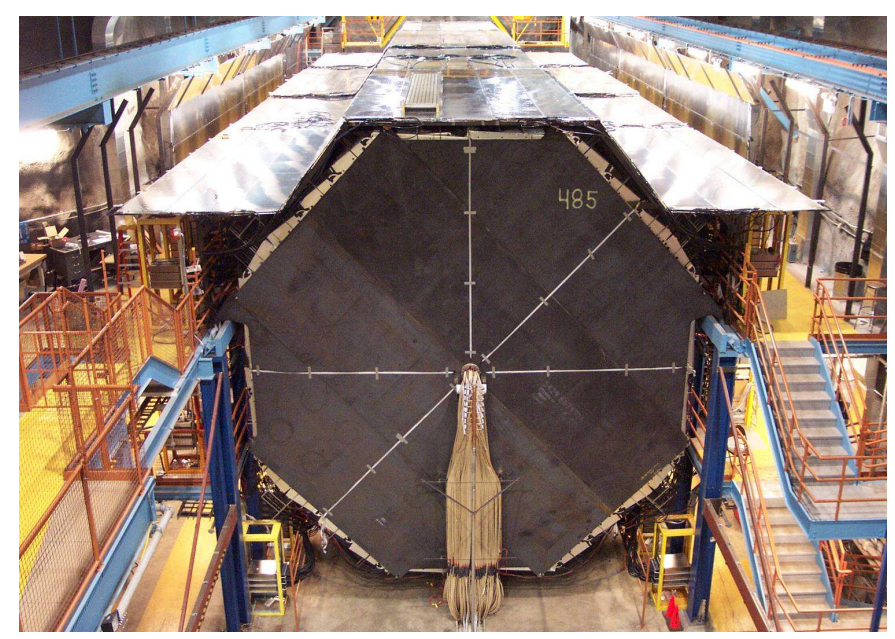

Figure 2.8: Downstream view of the MINOS Near Detector. Note the two electronics racks at the bottom of the view (about one meter tall) for sense of scale.

\subsubsection{FD triggers}

The activity triggers present in the Far Detector electronics are designed to record cosmic ray muons (present at the FD site at $\sim 0.5 \mathrm{~Hz}$ ) and beam neutrino events. The triggers are used to require a "minimally interesting" set of hits in the detector in order to record the activity, thus removing spurious noise from the PMTs and electronics, as well as low-activity events arising from radioactive decays in the rock surrounding the detector. The FD triggers are as follows, in decreasing order of importance for the beam analysis:

- Spill Trigger: The GPS time stamp of beam spills at Fermilab is sent via the Spill Server over networks lines to the Soudan Underground Lab. Because of the travel time (and transient network delays), the spill signal cannot reach the Far Detector before the neutrinos from the relevant spill. Therefore, a continuous block of digitized detector activity is stored in DAQ hardware registers until the time stamp attached to the activity can be compared to the beam spill time stamp. A window of $\sim 130 \mu \mathrm{s}$ is recorded for offline analysis, starting $\sim 40 \mu$ s before the expected arrival time of the start of the beam spill. This trigger is subdivided into true beam spills and "fake" spills, which allow for characterization of background detector activity during a spill window devoid of beam activity (see Section 4.5.1). 
- 4/5 plane trigger: In instances where the Spill Server fails (less than $1 \%$ of beam time), it is desirable to have redundant triggers that will record beam event activity in the absence of the spill signal. The $4 / 5$ plane trigger simply requires a hit in any four of five consecutive planes in the detector. This trigger is capable of recording nearly every beam event and has very high efficiency in recording muon tracks. This trigger is responsible for recording the cosmic ray muons in the time between beam spills.

- E4 trigger: In the event of a very low-energy neutrino-induced shower, the $4 / 5$ plane trigger may not be satisfied. In such instances, the E4 trigger will record any event in the detector with more than $1500 \mathrm{ADC}$ counts in any four contiguous planes and a total of at least six strip hits in two or more contiguous planes. This is sufficient to record low-energy electromagnetic and hadronic shower activity induced by a beam neutrino.

- Activity trigger: This trigger records activity in any 20 planes of the detector.

- Special triggers: These are present for special calibration runs and debugging purposes.

The recorded detector activity from a trigger is termed a snarl. In the Near Detector, one snarl typically contains 5-20 beam events due to the high intensity near the beam source; such a snarl must have events "sliced" in time to separate individual neutrino interactions. In the Far Detector, most snarls have only one event. Exceptions are due to reconstruction errors that slice one event into two or more pieces.

An extensive treatment of the module construction, detector layout, electronics, and calibration of the MINOS detectors can be found in Reference [44].

\subsection{FD event characteristics}

After detector activity digitization and offline event reconstruction, events in the MINOS Far Detector have distinct topologies that enable categorization by scanning or by a cut-based selection using reconstructed quantities. Cosmic ray muons (a potential background for beam rock muons), contained-vertex neutrino events, and rock-vertex muon events are all present in the data stream. It is useful to introduce these event types here. The event displays are all generated using the Mad package within the standard MINOS offline software package (see Section 4.2.3). 


\subsubsection{Cosmic ray muon events}

Cosmic rays illuminate the earth's atmosphere with roughly uniform intensity (ignoring the focusing effect of the geomagnetic field). The primary interactions of cosmic ray protons and heavy ions takes place in the upper atmosphere, causing a relativistic shower of hadrons, leptons, and photons. Only the decay products with the longest lifetimes and weakest interactions in the atmosphere survive to the earth's surface. Because of the uniform intensity of primary cosmic rays and the decay kinematics of the shower products, the intensity is highest at the local vertical (a zenith angle of $\theta_{z}=0^{\circ}$, where $\theta_{z}=90^{\circ}$ is the local horizon) and drops off towards the horizon. Cosmic ray muons (sometimes referred to as atmospheric muons) that reach the earth's surface must then penetrate a depth of rock (related to the vertical depth of the detector and $\theta_{z}$ ) to reach the MINOS Far Detector cavern. Muons that have enough energy to reach this depth lose energy as they pass through the detector. Please refer to Section 4.5 for a discussion of cosmic muons as a background to the desired NuMI beam events.

Figure 2.9 displays an example of a cosmic ray muon event. For reference throughout discussion of the event display, the six Panels (with numbering starting in the upper center panel and increasing counter-clockwise) represent the following pieces of information:

1. A rear view of the detector (along the positive $z$-axis) in the $x y$-plane. The four shorter sides of the detector are located at -4 and 4 meters in both $x$ and $y$. The four remaining sides are located at -4 and 4 meters in both $u$ and $v$.

2. A top view of the detector (along the positive $y$-axis) in the $x z$-plane. The scale will vary depending on the spatial extent of the event, but can run from -4 to 4 meters in $x$ and from 0 to 30 meters in $z$.

3. A side view of the detector (along the negative $x$-axis) in the $y z$-plane. The scale is analogous to Panel 2.

4. Two timing plots, the upper plot showing time against $y$ and the lower showing time against $z$. A negative/positive slope in the upper plot indicates a track moving down/up in the detector with time. A negative/positive slope in the lower plot indicates a track moving forward/backward in the detector with time. This is also referred to as moving downstream/upstream, in reference to the stream of neutrinos, which travel in the positive $z$-direction. 
5. A view of the detector (along the negative $u$-axis) in the $v z$-plane. The scale will vary depending on the spatial extent of the event, but can run from -4 to 4 meters in $v$ and from 0 to 30 meters in $z$.

6. A view of the detector (along the positive $v$-axis) in the $u z$-plane. The scale is analogous to Panel 5.

Panels 5 and 6 display the raw hit information, while Panels 1 - 4 display only hits that are reconstructed as part of the track (solid red points) or the shower (open yellow circles). In Panel 1, the light blue points outside of the detector represent hits in the veto shield.

We can dissect the anatomy of this event (Figure 2.9) as follows. Starting with Panel 4 , we see that the muon is traveling down with respect to the vertical direction, and upstream with respect to the horizontal. Any track with clear timing information that indicates movement upstream cannot result from a NuMI beam interaction, as all such events must travel away from Fermilab, in the positive $z$-direction. Since the muon flux is roughly uniform in azimuth (the angle measuring rotation about the positive $y$-axis) half of such muons will have this distinct timing topology and are easily removed from a sample of potential beam events. Since cosmic muons from the opposite side of the earth must penetrate the earth's entire diameter, virtually all such muons will range out within the mantle or core. This makes upward-going muons a very rare occurrence in MINOS. The NuMI beam points upward at $\sim 3^{\circ}$ at the location of the Far Detector, offering another hint of beam activity if a track is upward-going.

Since the muon is moving down and against the beam in time, Panel 1 shows us that the muon enters the $x=4 \mathrm{~m}$ side face, travels down and towards the front of the detector, then exits the $y=-4 \mathrm{~m}$ bottom face. Panels 2 and 3 show us the reconstructed track moving in the negative $x$ - and negative $y$-direction. The raw hit information in Panels 5 and 6 can indicate radiative losses by the muon, but often are the result of spurious detector activity or problems demultiplexing the strip origins. In this event, some of these spurious hits are reconstructed as part of the event shower. This is most apparent in Panel 5, which shows many of these hits separated from the track hits by $\sim 40 \mathrm{~cm}$ of little detector activity. 

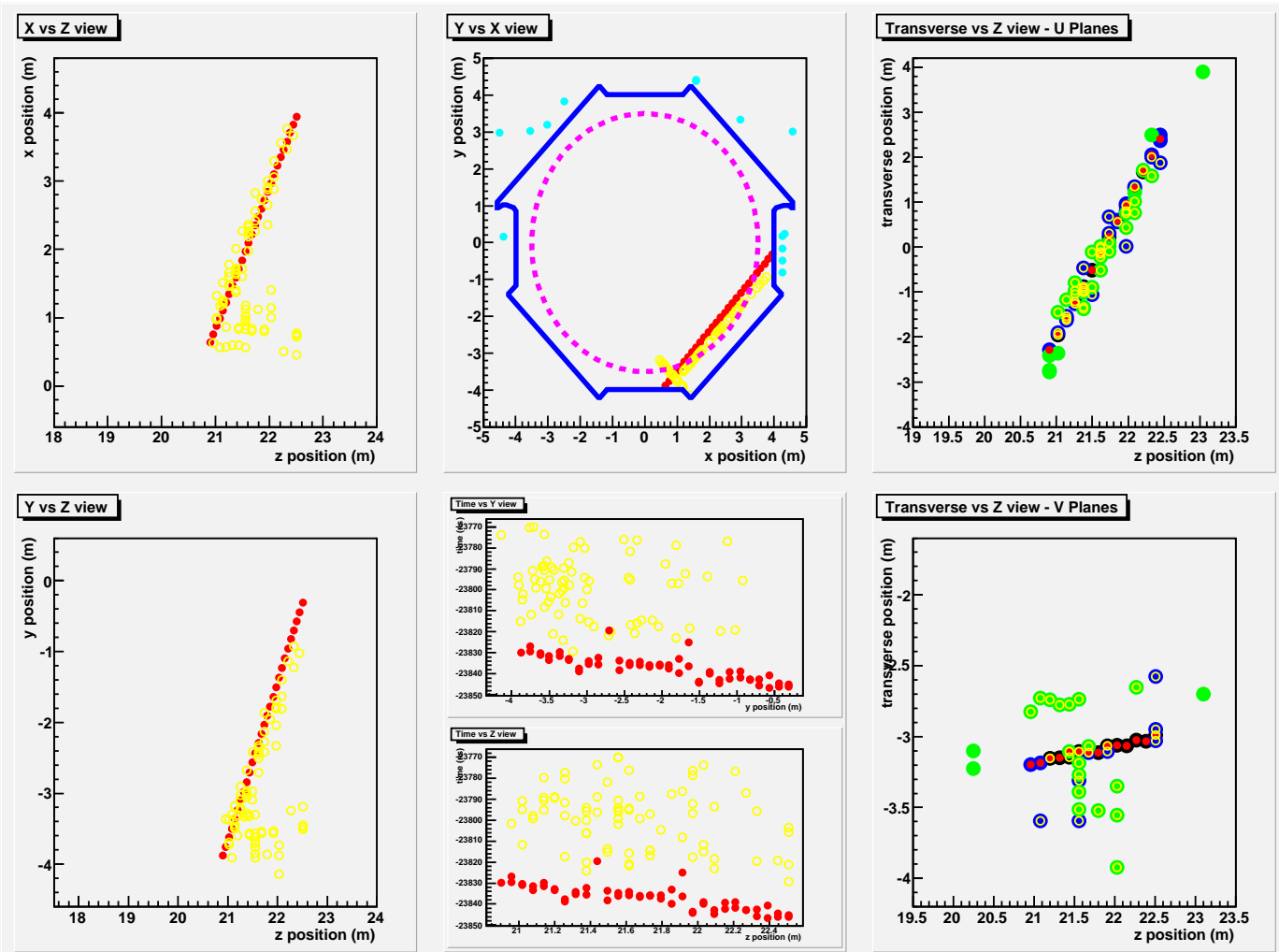

Figure 2.9: Example of a cosmic ray muon event: Run 29349, Snarl 54101.

\subsubsection{Contained vertex neutrino events}

Neutrinos that interact and deposit all of their energy in the fiducial volume of the Far Detector are the best type for the fiducial CC analysis[1]. Both neutral-current and charged-current DIS events will create a spray of hadron activity surrounding the interaction vertex. If the neutrino energy and interaction y-value (fraction of the neutrino energy carried by the muon) are large enough, a clean muon track will be visible, extending beyond the hadron shower structure for several meters. Charged-current QE events are conspicuous for their lack of shower activity. If properly reconstructed, chargedcurrent coherent pion production events are distinctive for their "two-prong" structure a short pion track accompanying the longer muon track.

An example of a charged-current deep inelastic scattering event with an interaction 
vertex within the fiducial volume of the detector (indicative of the event type selected in the contained-vertex sample used for the PRL analysis[1]) is shown in Figure 2.10. Panel 4 shows the muon to travel upward and downstream, consistent with a beam neutrino event. Panel 2 shows the muon curving towards the center $(x=0)$ of the detector. Under the "forward field" current configuration (the standard magnetic field orientation for the entire data sample considered here) this indicates a negatively charged muon. A significant amount of energy is deposited in the first several planes after the interaction vertex due to the hadron shower. The muon extends about six meters from the vertex. The event does not exit the detector by crossing any of the $x, y, u, v$, or $z$ boundaries and therefore is fully contained within the detector. In Panel 6, two parallel "ghost" tracks are evident, resulting from demultiplexing problems.
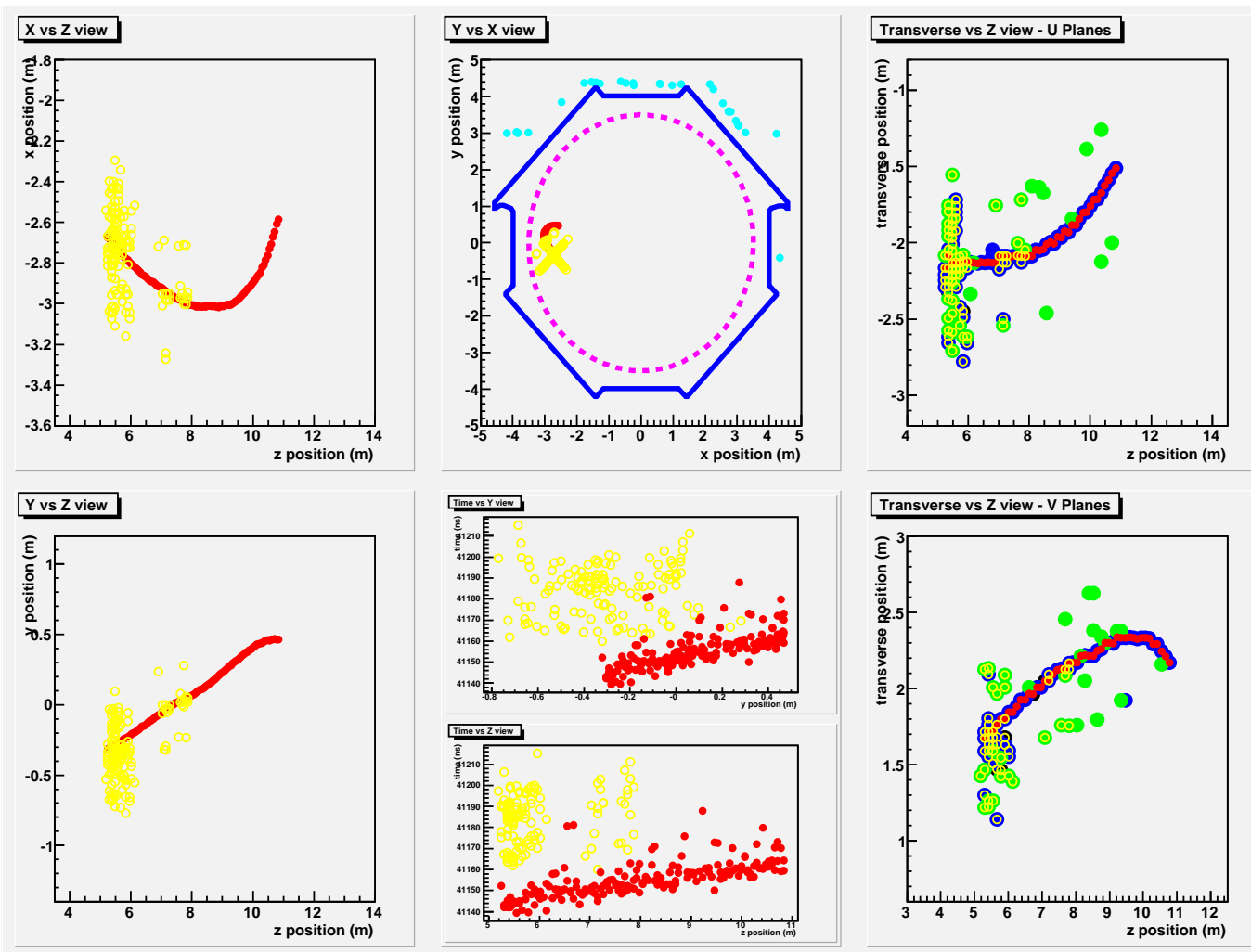

Figure 2.10: Example of a contained vertex CC DIS event: Run 32907, Snarl 102715. 


\subsubsection{Rock vertex muon events}

The main subject of this work is the rock muon. Neutrinos that interact in the rock surrounding the MINOS Far Detector cavern produce the same final state particles as when they interact inside of the detector; however, the rock effectively filters out the hadrons and admits only muons to penetrate large amounts of rock. In some instances, the neutrino interaction may take place close to the cavern wall, allowing hadron activity to reach the detector and contribute to the reconstructed event. The reconstructed tracks resulting from rock muons are typically very clean, with a stable ionization pattern. Multiple Coulomb Scattering for low-energy muons in the steel plates of the detector can wash out the expected curvature towards the end of the reconstructed track.

An example of a charged-current rock muon event that enters the front face of the Far Detector is shown in Figure 2.11. Panel 4 indicates the muon to be traveling upward and along the beam direction, consistent with expectation. In Panel 2, a scattering event is evident around $z=2.5 \mathrm{~m}$ that disturbs the curvature of the track towards the center of the detector. Ghost tracks appear to some extent in Panels 5 and 6, causing some of these cross-talk hits to be included in the reconstructed shower - the reconstruction algorithm "expects" to find hadron activity near the reconstructed track vertex, and so is vulnerable to errors of this type. Such errors have little affect this analysis, which uses only reconstructed track quantities in the fit procedure (see Chapter 6).

An example of a charged-current rock muon event that enters the side face of the Far Detector is shown in Figure 2.12. Panel 4 indicates timing consistent with a beam event, traveling up and along the beam direction. Panel 1 shows the entry point at the lower-left face of the detector along $u=-4 \mathrm{~m}$. The observed focusing of the muon towards the center of the detector indicates a negatively charged muon. Less cross-talk is present in this event, resulting in a cleaner track and almost no reconstructed shower activity.

\subsection{Blind analysis criteria}

In keeping with standard MINOS guidelines for charged current $\nu_{\mu}$ disappearance analyses, the work presented here was performed as a "blind analysis". The author has been exposed to various arguments as to the merits or flaws of such an approach. Essentially, the collaboration as a group desired to eliminate any bias present that MINOS should measure the atmospheric neutrino oscillation parameters to agree with prior $\mathrm{K} 2 \mathrm{~K}$ or 

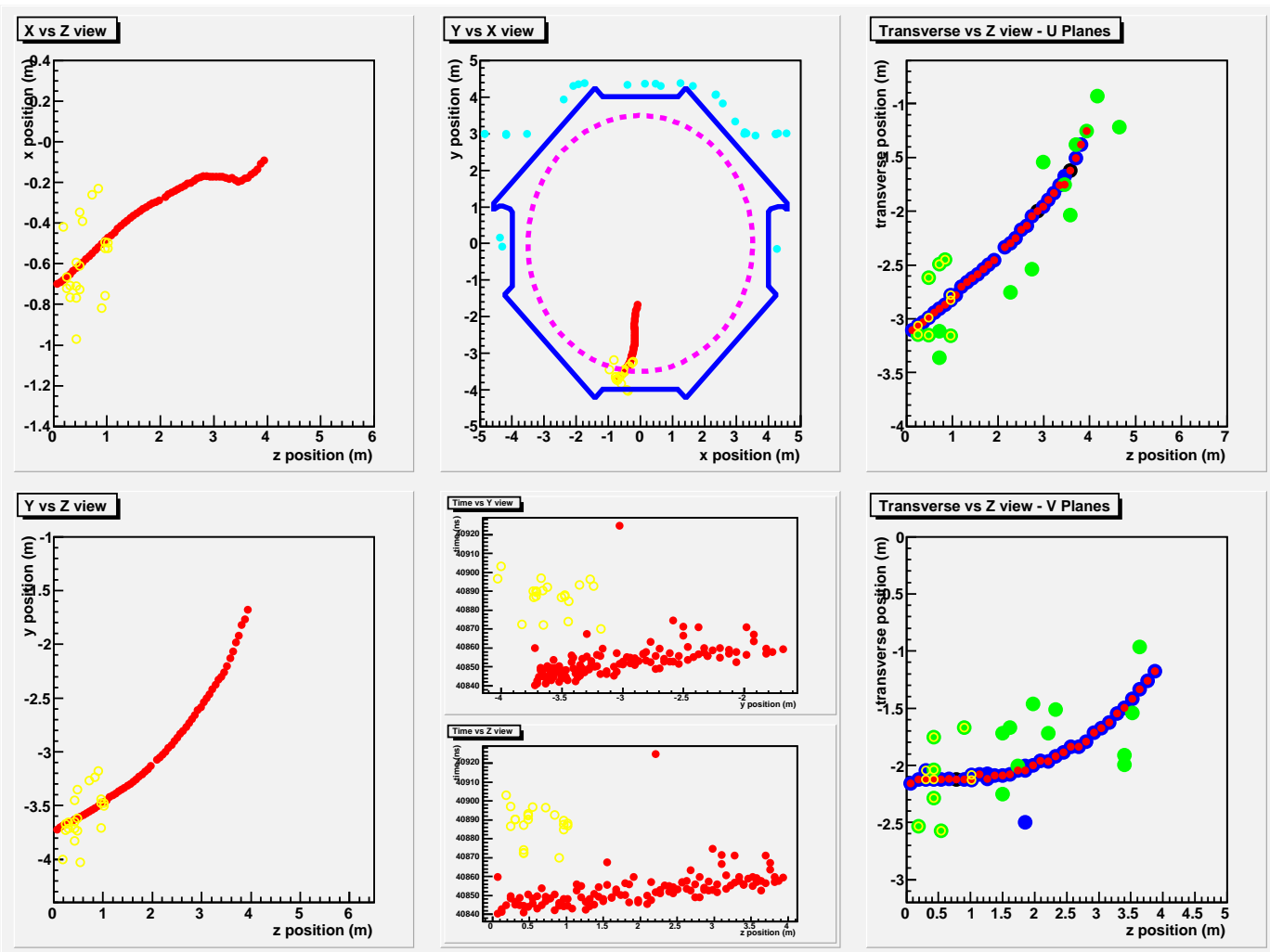

Figure 2.11: Example of a selected rock muon event penetrating the front of the detector: Run 33452, Snarl 48800.

Super-K results.

In MINOS, the blinding was accomplished by one collaborator implementing a blinding function, which removed some fraction of Far Detector events in an energy-dependent manner. This "blinded" data stream was provided to all collaborators in order to ensure that the recorded event rates and various reconstructed quantities were stable over time. The blinding function effectively prevented any premature attempt to measure the oscillation parameters, as the unknown energy dependence masked the true disappearance rate.

The original "unblinded" data stream was recorded and made available to analysts when they had demonstrated competency using "mock data" Monte Carlo samples (see 


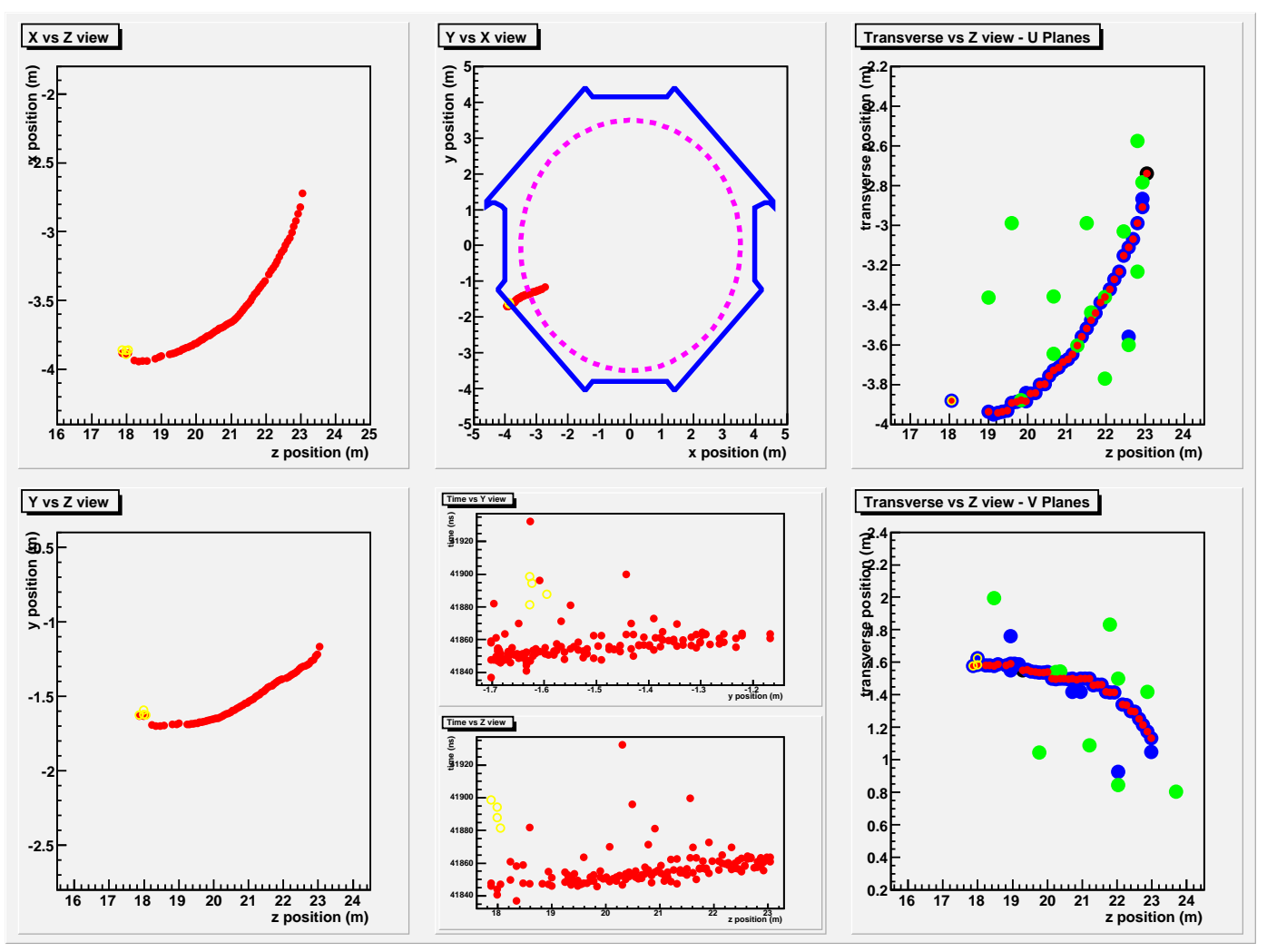

Figure 2.12: Example of a selected rock muon event penetrating the side of the detector: Run 29349, Snarl 54101.

Chapter 6). These samples were prepared by one collaborator who chose input neutrino oscillation parameters. They were then provided to analysts (uninformed of the input parameters) who would perform their analysis and extract the unknown parameters. When this procedure was complete, and the analyst presented a solid estimate of systematic uncertainties, the analysis was given "box-opening" approval. At this stage, the unblinded data was used to obtain a likelihood of the no-disappearance hypothesis. When this hypothesis was ruled out, a measurement of the oscillation parameters was carried out, under the assumption of the oscillation hypothesis.

The author received approval for box-opening at the MINOS Collaboration Meeting in June 2007. Every aspect of the analysis presented here was finalized before the boxopening took place, and the resulting measurement has not been altered in any way. All 
work presented here is that of the author, unless specifically attributed to others. Several pictures and figures have been used from the MINOS document repository. 


\section{Chapter 3}

\section{Monte Carlo simulation}

A computer lets you make more mistakes faster than any invention in human history - with the possible exceptions of handguns and tequila.

-Mitch Ratliffe

Several physics simulation packages contribute to the final Monte Carlo product used in this analysis. The simulated interactions of the proton beam on the NuMI target and associated hadron production are handled in FLUKA[45], while the tracking of these hadrons through the focusing horns and beam line is handled in GNuMI[46] (a GEANT3based model). The neutrino interactions in and around both the Near and Far Detectors are modeled in GEANT3[47], with their respective underground halls and surrounding rock included as target material in the simulation. This detector simulation is accomplished with a specific package of code called GMINOS[48] within the larger labyrinth[49] package. The labyrinth is a FORTRAN-based package that also includes neugen[50], a physics simulation of neutrino events based on a comprehensive cross section model.

Due to the nature of the extrapolation method used in this analysis, which involves measuring the neutrino event spectrum at the ND and using this to predict the muon event spectrum at the FD, the modeling of rock vertex events at the ND was not used since the ND rock muon spectrum does not inform the fit to FD events. In Section 3.2, the author's model of the rock surrounding the FD is presented, as this is the important region for properly determining the flux of muons coming from the rock at the FD. The conclusions based on this model were used to adjust the relevant GMINOS parameters that went into the final analysis.

In accordance with MINOS tradition, the generations of Monte Carlo are labeled with 
names of vegetables. The earliest incarnations included Avocado and Beet. The version used for this analysis is named Carrot. Generation of rock events in Daikon release has not been processed. Any reference herein to improvements to the GMINOS rock model resulting from the author's investigations will be implemented in Eggplant release and beyond, with the exception of the expanded rock volume, which was implemented starting with Carrot release.

\subsection{Target and beam line}

The NuMI target is modeled in FLUKA, a dedicated hadron production software package. The hadrons output from FLUKA are fed into GNuMI, which propagates and decays the relevant parent and daughter particles through the magnetic fields in the focusing horns and the decay pipe. The final output of this procedure is packaged in a flux file, which can be fed directly into GMINOS for simulation of the detectors and surrounding rock. The most recently used flux files are Versions 18 and 19. Details on the target and water cooling line geometry (as existing in V.18) can be found in Reference [51] Carrot Monte Carlo was produced using V.18 flux files, while Daikon was produced using V.19 files. Output hadron spectra were quite similar between the two versions, with a few bug fixes and improvements going into V.19[52].

A list of the particle decays used in FLUKA/GNuMI that gives rise to the neutrino beam is provided in Table 3.1, taken from the MINOS TDR[53] with updated values from the PDG tables[54]. Average proper lifetimes are used to obtain $\mathrm{c} \tau$, the average distance traveled in the hadron rest frame before decay. Only leptonic and semi-leptonic decay modes that directly produce a neutrino are listed. Hadronic modes, such as $K^{+} \rightarrow \pi^{+} \pi^{0}$ $(\mathrm{BR}=21.13 \%)$, result in secondary neutrino production from the pion decays listed.

A major uncertainty in this analysis is the absolute flux constraint on $\nu_{\mu}$ arising from kaon production in the NuMI beam line. This is due to the higher average energy of muon neutrinos from kaon decay (see Appendix B.2), which contribute a substantial fraction of the rock muon sample (about $30 \%$ of selected events below $30 \mathrm{GeV} / \mathrm{c}$ ). Following the recommendation of the beam systematics group[55], a value of $20 \%$ for the uncertainty in the $K / \pi$ ratio is adopted for this analysis. The contributions to the selected muon sample from the pion and kaon parents are separated, and motivation for allowing these two contributions to have separate normalization in the oscillation fit is developed in Section 6.1.2. 


\begin{tabular}{|l|c|c|}
\hline \hline Decay mode & $\mathrm{c} \tau(\mathrm{m})$ & Branching Ratio (\%) \\
\hline$\pi^{+} \rightarrow \nu_{\mu} \mu^{+}$ & 7.805 & 100 \\
$\pi^{-} \rightarrow \bar{\nu}_{\mu} \mu^{-}$ & " & 100 \\
$K^{+} \rightarrow \nu_{\mu} \mu^{+}$ & 3.713 & 63.44 \\
$K^{+} \rightarrow \nu_{e} e^{+} \pi^{0}$ & " & 4.98 \\
$K^{+} \rightarrow \nu_{\mu} \mu^{+} \pi^{0}$ & " & 3.32 \\
$K^{-} \rightarrow \bar{\nu}_{\mu} \mu^{-}$ & " & 63.44 \\
$K^{-} \rightarrow \bar{\nu}_{e} e^{-} \pi^{0}$ & " & 4.98 \\
$K^{-} \rightarrow \bar{\nu}_{\mu} \mu^{-} \pi^{0}$ & " & 3.32 \\
$K_{L}^{0} \rightarrow \nu_{e} e^{+} \pi^{-}$ & 15.33 & 20.26 \\
$K_{L}^{0} \rightarrow \bar{\nu}_{e} e^{-} \pi^{+}$ & " & 20.26 \\
$K_{L}^{0} \rightarrow \nu_{\mu} \mu^{+} \pi^{-}$ & " & 20.26 \\
$K_{L}^{0} \rightarrow \bar{\nu}_{\mu} \mu^{-} \pi^{+}$ & " & 13.51 \\
$\mu^{-} \rightarrow \nu_{\mu} \bar{\nu}_{e} e^{-}$ & 658.654 & 100 \\
$\mu^{+} \rightarrow \bar{\nu}_{\mu} \nu_{e} e^{+}$ & " & 100 \\
\hline \hline
\end{tabular}

Table 3.1: Summary of particle decays in the NuMI beam line as modeled in GNuMI.

Various particle numbering schemes have been introduced over the years, serving as a source of confusion when coupling different Monte Carlo stages. Table 3.2 displays the codes for FLUKA, GNuMI, and GMINOS (which uses standard PDG codes) as compiled from the GNuMI documentation[46] and the FLUKA user manual[45].

\subsection{Basic rock Monte Carlo}

The author's early investigations into the sensitivity of a measurement of the oscillation parameters involved the construction of a model of the Far Detector and surrounding rock. This model included a rectangular box with the same front and side face areas and length along the beam direction as that of the MINOS FD. It also included enough rock upstream of the detector to range out a $120 \mathrm{GeV}$ muon, as this was the initial energy of the accelerated primary protons in the NuMI beam line. The model used as input a flux of muons lifted from the GMINOS interactions in the FD. These fourvectors (energy and spatial momentum) were then randomly placed in the rock volume surrounding the detector box. This step assumed the equality of the cross sections for charged current interactions in the rock and in the detector. Using an assumed constant energy loss along the path of each muon, a fixed energy was removed in each step and a determination of a detector "hit" was made if the muon had energy remaining when it 
reached the detector. If the muon intersected the detector box, its remaining energy and incident angle was recorded in an idealized "reconstructed" spectrum, which resembles the true event spectrum seen in GMINOS. Multiple scattering was not accounted for in this model, nor were radiative energy losses.

From this simple model, initial estimates of the expected muon rates under various oscillation hypotheses were formed and the sensitivity of a disappearance analysis was estimated. Once the concept was validated, the full GMINOS simulation for the Monte Carlo prediction of the muon spectrum was used for the analysis.

\subsubsection{Rock density}

One issue in the construction of the model was the modeling of the air gaps in the Far Detector cavern. GMINOS accurately represents this space, filled with air in the simulation. The model did not attempt to represent the cavern dimensions in any way, such that the detector box was completely enclosed in the surrounding rock. This was justified by the fact that the extra mass (in the model) intervening in this empty space (in the true FD cavern) provided a greater absorption of muon energy as well as a greater number of created muon events, both proportional to the density increase in this volume. To first order, these two effects cancel each other. When detection and reconstruction efficiencies were taken into account, no significant difference in the muon rates was found when comparing the spectra arising from the author's model and the GMINOS model.

Another issue was the rock density in the model. The density as modeled in GMINOS

was $2.5 \mathrm{~g} \mathrm{~cm}^{-3}$. Rock survey measurements of the Greenstone representative of the rock surrounding the Far Detector indicated an average density of $2.8 \mathrm{~g} \mathrm{~cm}^{-3}$. This density parameter has since been updated in the GMINOS code and will be used for all future generation (Eggplant and beyond) of rock vertex neutrino interactions. For the purpose of this analysis, which used Carrot-era Monte Carlo, it was necessary to demonstrate that this density difference would not affect the muon rate normalization in a significant way.

This demonstration was achieved by simply changing the rock density input to the Monte Carlo and tabulating the resulting event rates. The results of this study are summarized in Table 3.3. All event rates are scaled down from the absolute rate achieved in the model in order to reflect the reconstruction and selection efficiency in the full GMINOS simulation. Specifically, the model rates are all normalized to the rate at $\rho_{\text {rock }}=2.5 \mathrm{~g} \mathrm{~cm}^{-3}$, the value used in the GMINOS model. The observed fluctuations in 
the event rate are consistent with the size of the statistical uncertainty $(0.2 \%)$.

\subsubsection{Muon energy loss}

Another approximation in this model was to use a representative muon $d E / d X$ at 10.0 $\mathrm{GeV}$ as a constant value along the entire path, as opposed to using an energy-dependent value for the energy loss. For muons below the critical energy (above which radiative processes contribute significantly) this energy loss is almost entirely due to ionization and atomic excitation of the absorbing material. The typical critical energy for muons, which depends on the material traversed, falls in the $200-400 \mathrm{GeV}$ range. Energy loss characteristics have been measured extensively for muons passing through various bulk media. The standard measurement is reported as $d E / d X$ (in $\mathrm{GeV} \mathrm{cm}^{2} \mathrm{~g}^{-1}$ ); when multiplied by the mass density $\left(\mathrm{g} \mathrm{cm}^{-3}\right)$ this provides the energy loss per unit of distance traveled $\left(\mathrm{GeV} \mathrm{cm}^{-1}\right)$. This average rate of energy loss is described[54] by the Bethe-Bloch equation:

$$
-\frac{d E}{d X}=K z^{2} \frac{Z_{a v g}}{A_{a v g}} \frac{1}{\beta^{2}}\left[\frac{1}{2} \ln \frac{2 m_{e} c^{2} \beta^{2} \gamma^{2} T_{\max }}{I^{2}}-\beta^{2}-\frac{\delta(\beta \gamma)}{2}\right],
$$

where $K=4 \pi N_{A} r_{e}^{2} m_{e} c^{2}, N_{A}$ is Avogadro's number, $r_{e}$ is the classical electron radius, $z$ is the charge of the particle in units of $e$ ( $z=1$ for muons), $m_{e} c^{2}$ is the rest energy of

an electron $\left(5.11 \times 10^{-4} \mathrm{GeV}\right), Z_{\text {avg }}$ is the average atomic number of the material, $A_{\text {avg }}$ is the average atomic mass, $\beta=v / c$ is the speed of the muon, $\gamma=\left(1-\beta^{2}\right)^{-1 / 2}$ is the relativistic gamma factor, $T_{\max }$ is the maximum kinetic energy that a free electron can obtain in a single collision, $I$ is the mean excitation energy in $\mathrm{eV}$, and $\delta(\beta \gamma)$ is the density effect correction to ionization energy loss.

For muons traveling in standard rock, the ionization term dominates up to $\sim 200 \mathrm{GeV}$. Since the upper limit on muon energy for beam-induced events is $120 \mathrm{GeV}$, it is safe to ignore the processes of bremsstrahlung, pair production, and photo-nuclear interaction in the model. While these processes are all modeled in the full GMINOS simulation, they contribute $\sim 3 \%$ of the total energy loss for the highest energy muons and only $\sim 0.5 \%$ at a representative muon energy of $10 \mathrm{GeV}$. Figure 3.1 displays the ionization and total energy loss in the energy range relevant for NuMI beam interactions. These figures have been generated using the MuELoss software package[56] developed by C. Andreopoulos. MuELoss uses the Bethe-Bloch model for ionization, the Bethe-Heitler and Petrukhin-Shestakov models for bremsstrahlung, the Kokoulin-Petrukhin model for 
electron/positron pair production, and the Bezrukov-Bugaev model for photo-nuclear interactions.

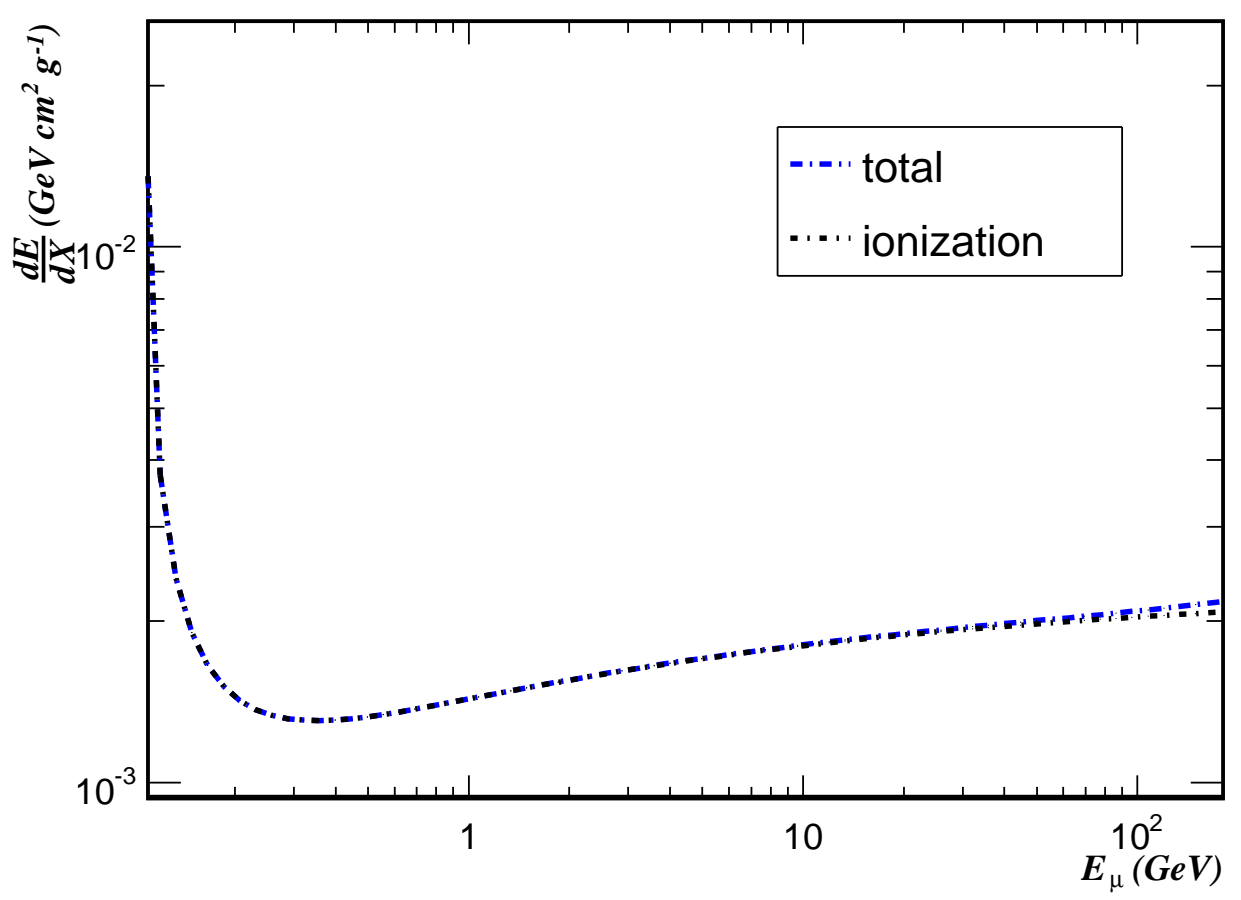

Figure 3.1: Muon energy loss in standard rock for energies below $120 \mathrm{GeV}$, dominated by the ionization term.

As seen in Figure 3.1, the curve bottoms out at $1.4 \times 10^{-3}$ (near $0.3 \mathrm{GeV}$, towards the end of the muon path) then slowly increases to $2.0 \times 10^{-3}$ (near $100 \mathrm{GeV}$ ). For the model, a value of $1.9 \times 10^{-3} \mathrm{GeV} \mathrm{cm} \mathrm{g}^{-1}$ (near $10 \mathrm{GeV}$, close to the average muon energy as created in the rock) was used, and a value of $2.80 \mathrm{~g} \mathrm{~cm}^{-3}$ was used for the rock density, obtained as a representative value from various studies of the rock composition and density compiled by K. Ruddick[57]. The product used in this model was then $5.32 \times 10^{-3} \mathrm{GeV} \mathrm{cm}^{-1}$.

Muon energy loss varies with the composition of the material being traversed. Figure 3.2 displays the total energy loss curve for pure iron alongside the curve for standard rock. The energy loss rates are very similar below $30 \mathrm{GeV}$. The loss rate is everywhere 
higher in iron than in standard rock, and begins to climb at a greater rate at high energy due to the increased contributions from radiative processes.

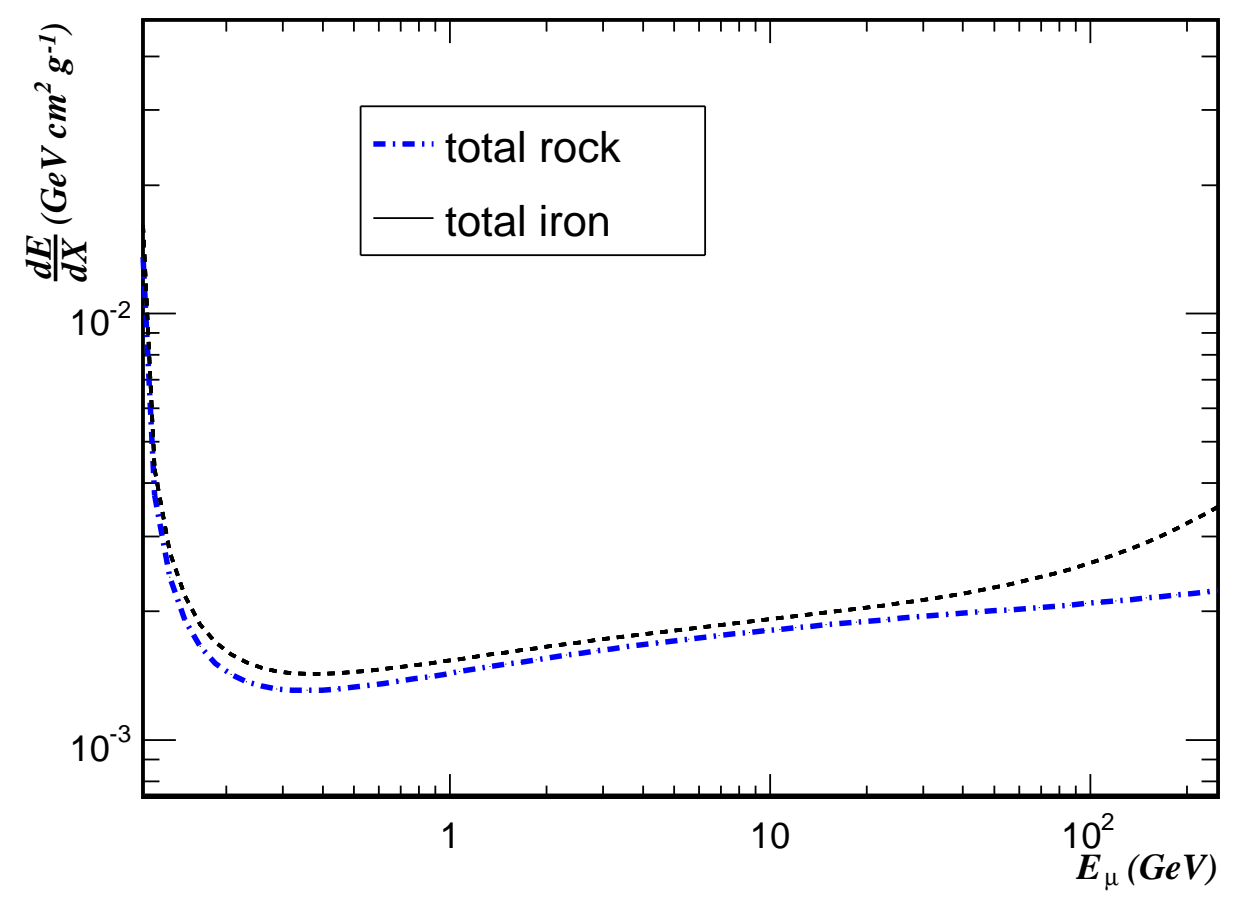

Figure 3.2: Muon energy loss in iron compared with loss in standard rock.

\subsubsection{Rock volume studies}

One can apply a safe upper limit on the energy of any rock muon to be equal to the initial NuMI proton energy of $120 \mathrm{GeV}$. The effectively infinite volume of rock necessary to simulate the full muon spectrum at the FD was modeled with $\sim 300 \mathrm{kT}$ of rock surrounding the detector cavern, roughly 60 times the FD mass.

Given the continuous energy loss assumption and this upper bound on the muon energy, the required rock thickness (upstream of the detector cavern) to range out such 
a muon is given by

$$
\text { range }=\frac{E_{\mu}^{\max }}{<d E / d X>\rho_{\text {rock }}} \sim 225 \text { meters. }
$$

The other relevant parameter in this volume study is the transverse thickness of rock necessary on the sides of the detector. Since a wide range of muon energies and angles are present in the event sample, there is no analytic calculation to find a "safe" value for this rock thickness. A simple approach would be to model $225 \mathrm{~m}$ of transverse rock, enough to range out the highest possible energy muon approaching the detector from the transverse direction (a fanciful feat). The author's studies indicate that every detectable Monte Carlo rock muon event as generated by GMINOS would be produced within a rock radius of $114 \mathrm{~m}$; however, such a large volume is taxing on the available computing resources. By adjusting this parameter in the author's model and recording the calculated muon rates, a plateau was found at $8 \mathrm{~m}$ of rock at which point $95 \%$ of the maximum recorded rate was reached. Table 3.4 displays the relative rates at various levels of transverse rock thickness.

Due to tight constraints on Monte Carlo generation and processing time during the Carrot era, the GMINOS value for this parameter was chosen to be eight meters. This decision was a compromise between the author's volume study and another similar study, which showed a $1 \%$ rate increase in going from $5 \mathrm{~m}$ of transverse rock to $12 \mathrm{~m}$. The author's study agreed roughly with this observed increase; however, the other study (using GMINOS) did not model any values beyond $12 \mathrm{~m}$. Due to the time constraints, the Monte Carlo group settled on a value of $8 \mathrm{~m}$ for Carrot generation. In future MC generation, the author will campaign for a value closer to $20 \mathrm{~m}$ of transverse rock in the GMINOS model, which achieves $\sim 99 \%$ of the expected rate at infinite $(114 \mathrm{~m}$ of transverse rock) volume in the author's Monte Carlo model. However, there will always be a trade-off between required CPU resources and the desire to model the fullest possible muon spectrum. Figure 3.3 shows the neutrino interaction vertex location for selected muon events as modeled in GMINOS. The upstream wall, floor, and ceiling of the detector cavern are visible.

At the time, it was realized that a correction may be required in order to accommodate the deficiencies at the outer regions of the GMINOS rock model. This correction would effectively involve a reweighting of muon events that arise from the high energy tail of the neutrino spectrum. As described in Section 6.1.2, the author's approach was to separate the neutrino contributions from pions and kaons. Since the kaon contribution 


\section{rock vertex position}

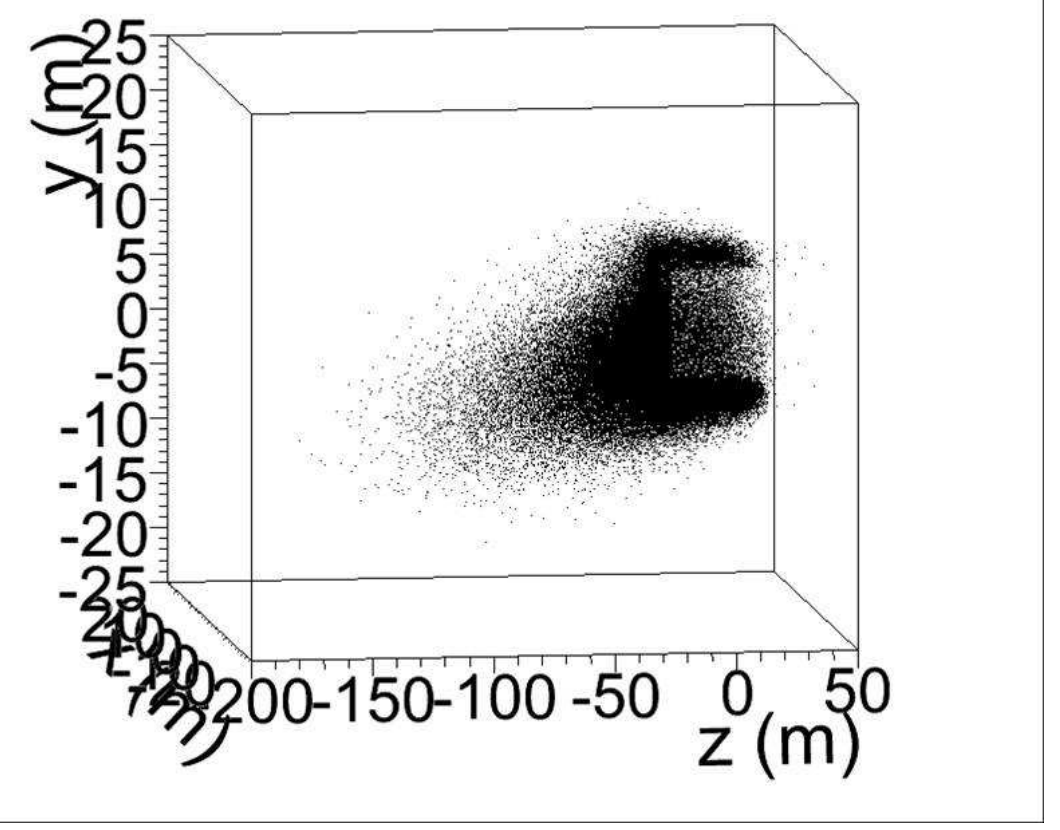

Figure 3.3: True neutrino interaction vertex locations as modeled in GMINOS for rock muon events passing Far Detector selection. The positive $\mathrm{x}$-axis is into the page, the positive $y$-axis to the top of the page, and the positive z-axis to the right. Notice the upslope of the beam, which causes more muon events to enter the bottom of the detector.

arises primarily from neutrinos above $30 \mathrm{GeV}$, this template could be adjusted upward to account for the missing rock mass at the outer limits of the GMINOS model. This template normalization scheme takes into account our uncertainty in the $\mathrm{K} / \pi$ flux ratio used in the beam model as mentioned in Section 3.1. By allowing a renormalization of the muons arising from kaon-parented neutrinos, the expected rate of muons coming from the "missing" mass at the outer edges of the GMINOS model could be recovered in the final prediction. 


\subsubsection{Multiple Coulomb scattering}

Muons passing through the rock surrounding the MINOS Far Detector cavern undergo multiple Coulomb scattering (MCS) from the electrons and nuclear electric fields encountered in the medium[54]. MCS was included in the GMINOS model and studied briefly in the author's Monte Carlo model. The ultimate effect of muon scattering within the rock is to deflect some muons away from the detector that had an initial momentum pointing towards the detector and to deflect other muons towards the detector that would otherwise not have intersected the detector. To the precision of the author's study, these effects cancel each other.

Multiple Coulomb scattering scrambles the initial direction information that would have been present had the muon continued on a straight path from its creation point in the rock. In this sense, MCS is another contributing factor that destroys information about the original neutrino energy, since the energy and angle of the muons created are correlated to the parent $E_{\nu}$.

When the effects of MCS are modeled in a consistent way within GMINOS, one can compare the angle of the created muon with respect to the initial neutrino direction with the angle of the muon as it enters the MINOS Far Detector. When these are compared for an ensemble of rock muon events, the mean deviation from the original direction for muons arising from NuMI beam interactions is found to be $14^{\circ}$, with an RMS of $7^{\circ}$.

\subsection{Soudan rock composition}

The Soudan mine is located in an iron-rich rock formation. The majority of this formation is composed of an igneous rock named Greenstone. The composition and density of the Soudan rock varies significantly throughout the mine structure. Additionally, the geometry of hollowed mine drifts and access shafts is complex throughout the overburden and downstream regions of rock; however, the rock upstream of the MINOS detector has not been mined and effectively satisfies the "infinite upstream medium" considered in Section 1.4.2.

A study using cosmic muon intensity as a function of direction, measured with the Soudan 2 detector, were performed by S. Kasahara[58]. This study is relevant to MINOS models because the Soudan 2 detector was located in an adjacent cavern on the same level as the MINOS Far Detector. Kasahara's results indicate an average density of $2.8 \mathrm{~g} \mathrm{~cm}^{-3}$ upstream of the MINOS Far Detector, with directional variations on the 
order of $5-10 \%$. For modeling purposes, it has been shown that the input rock density has no statistically significant effect on the measured event rate (see Table 3.3).

The rock composition will affect the measured event rate through a second-order effect arising from nuclear corrections to cross sections and difference in muon energy loss within the rock. The muon energy loss arising from ionization is proportional to the average $Z / A$ of the material, which varies little across a wide range of rock types. Table 3.5 displays the differences between Soudan rock and the rock as modeled in GMINOS. The $\langle Z\rangle /\langle A\rangle$ values are the same to within $\sim 1 \%$. The main difference between the Monte Carlo model and the measured Greeenstone samples lies in the effective $\langle Z\rangle^{2} /\langle A\rangle$ value, which the radiative energy losses (bremsstrahlung, pair production, etc.) are proportional to. While these differ by $8 \%$, the radiative losses are negligible at the relevant muon energies arising from NuMI neutrino interactions in the rock (see Figure 3.1). Because the beam-induced muons have very little radiative energy loss, only the $\langle Z\rangle /\langle A\rangle$ difference (affecting the ionization energy loss) is treated as a source of uncertainty for this analysis.

Tables 3.6 and 3.7 list the major contributing elements and their percent contributions to the composition of the Greenstone rock and the rock as modeled in GMINOS for Carrot-era Monte Carlo production. As a result of Ruddick's compilation[57] in Table 3.6 and the author's request to the Monte Carlo working group, this composition has been adopted for all future generations of GMINOS, beginning with Eggplant. The effect of the composition differences between the rock upstream of the MINOS Far Detector and the Carrot-era GMINOS model was estimated using the author's Monte Carlo rock model. The $1 \%$ difference in $Z / A$ between the Greenstone survey and the GMINOS rock model is taken as the total uncertainty in $Z / A$ used in the model. The average change in the total event rate by introducing shifts of $\pm 1 \%$ in the total energy loss (encompassing the difference between the GMINOS model and Soudan rock, as well as our uncertainty in the Soudan rock composition) is found to be $1.6 \%$. This uncertainty is discussed in Section 6.6.3. The cross section uncertainty is addressed in Section 6.6.2. 


\begin{tabular}{|l|l|l|l|}
\hline \hline Particle & Fluka & GNuMI & GMINOS (PDG) \\
\hline$p^{+}$ & 1 & 14 & 2212 \\
$p^{-}$ & 2 & 15 & -2212 \\
$e^{-}$ & 3 & 3 & 11 \\
$e^{+}$ & 4 & 2 & -11 \\
$\nu_{e}$ & 5 & 53 & 12 \\
$\bar{\nu}_{e}$ & 6 & 52 & -12 \\
$\gamma$ & 7 & 1 & 22 \\
$n$ & 8 & 13 & 2112 \\
$\bar{n}^{+}$ & 9 & 25 & -2112 \\
$\mu^{+}$ & 10 & 5 & -13 \\
$\mu^{-}$ & 11 & 6 & 13 \\
$K_{L}^{0}$ & 12 & 10 & 130 \\
$\pi^{+}$ & 13 & 8 & 211 \\
$\pi^{-}$ & 14 & 9 & -211 \\
$K^{+}$ & 15 & 11 & 321 \\
$K^{-}$ & 16 & 12 & -321 \\
$K_{S}^{0}$ & 19 & 16 & 310 \\
$\pi^{0}$ & 23 & 7 & 111 \\
$K^{0}$ & 24 & $10+16$ & 311 \\
$\bar{K}^{0}$ & 25 & $10+16$ & -311 \\
$\nu_{\mu}$ & 27 & 56 & 14 \\
$\bar{\nu}_{\mu}$ & 28 & 55 & -14 \\
$\tau^{+}$ & 41 & 33 & -15 \\
$\tau^{-}$ & 42 & 34 & 15 \\
$\nu_{\tau}$ & 43 & 59 & 16 \\
$\bar{\nu}_{\tau}$ & 44 & 58 & -16 \\
\hline \hline
\end{tabular}

Table 3.2: Cross reference for common Particle ID codes used in MINOS Monte Carlo. 


\begin{tabular}{|c|c|}
\hline \hline $\begin{array}{c}\text { Density } \\
\left(\mathrm{g} \mathrm{cm}^{-3}\right)\end{array}$ & $\begin{array}{c}\text { Selected events 1.27e20 POT } \\
\pm 0.56 \text { (statistical uncertainty) }\end{array}$ \\
\hline 2.3 & 251.62 \\
2.4 & 251.35 \\
2.5 & 252.90 \\
2.6 & 251.83 \\
2.7 & 251.31 \\
2.8 & 250.82 \\
2.9 & 251.38 \\
3.0 & 252.87 \\
\hline \hline
\end{tabular}

Table 3.3: Summary of simulated rock density changes in basic Monte Carlo. The calculated fluctuations in the muon rate are consistent with the statistical uncertainty (see Section 3.2.1).

\begin{tabular}{|c|c|}
\hline \hline Transverse rock $(\mathrm{m})$ & Event rate \\
\hline 2.0 & $235.5 \pm 1.0$ \\
4.0 & $243.8 \pm 1.0$ \\
6.0 & $245.5 \pm 1.0$ \\
8.0 & $252.9 \pm 1.1$ \\
10.0 & $256.3 \pm 1.1$ \\
15.0 & $256.9 \pm 1.1$ \\
20.0 & $266.0 \pm 1.1$ \\
60.0 & $264.8 \pm 1.1$ \\
\hline \hline
\end{tabular}

Table 3.4: Event rate for $1.27 \times 10^{20}$ POT as a function of transverse rock thickness in Monte Carlo model.

\begin{tabular}{|c|c|c|}
\hline \hline Sample & Avg. Z/A & Avg. Z ${ }^{2} / \mathrm{A}$ \\
\hline Greenstone & 0.4902 & 6.111 \\
GMINOS & 0.4964 & 5.619 \\
\hline \hline
\end{tabular}

Table 3.5: Comparison of composition between Greenstone and GMINOS rock. 


\begin{tabular}{|c|c|c|c|}
\hline \hline Element & $\mathrm{Z}$ & $\mathrm{A}$ & \% by weight \\
\hline $\mathrm{O}$ & 8 & 15.999 & 45.86 \\
$\mathrm{Si}$ & 14 & 28.086 & 23.70 \\
$\mathrm{Fe}$ & 26 & 55.845 & 8.56 \\
$\mathrm{Al}$ & 13 & 26.982 & 7.97 \\
$\mathrm{Ca}$ & 20 & 40.078 & 6.41 \\
$\mathrm{Mg}$ & 12 & 24.305 & 3.95 \\
$\mathrm{Na}$ & 11 & 22.990 & 1.90 \\
$\mathrm{Ti}$ & 22 & 47.867 & 0.64 \\
$\mathrm{~K}$ & 19 & 39.098 & 0.34 \\
$\mathrm{H}$ & 1 & 1.008 & 0.26 \\
$\mathrm{C}$ & 6 & 12.011 & 0.20 \\
$\mathrm{Mn}$ & 25 & 54.938 & 0.15 \\
$\mathrm{P}$ & 15 & 30.974 & 0.06 \\
\hline Total/Avg. & 12.465 & 25.426 & 100.0 \\
\hline \hline
\end{tabular}

Table 3.6: Composition of Greenstone rock, the main type found in the region surrounding the Soudan mine.

\begin{tabular}{|c|c|c|c|}
\hline \hline Element & $\mathrm{Z}$ & $\mathrm{A}$ & \% by weight \\
\hline $\mathrm{O}$ & 8 & 15.999 & 53.00 \\
$\mathrm{Si}$ & 14 & 28.086 & 33.00 \\
$\mathrm{Ca}$ & 20 & 40.078 & 6.30 \\
$\mathrm{Al}$ & 13 & 26.982 & 4.20 \\
$\mathrm{Fe}$ & 26 & 55.845 & 2.00 \\
$\mathrm{Na}$ & 11 & 22.990 & 1.20 \\
\hline Total/Avg. & 11.318 & 22.799 & 100.0 \\
\hline \hline
\end{tabular}

Table 3.7: Composition of rock as modeled in GMINOS simulation. 


\section{Chapter 4}

\section{Event selection}

Children are born true scientists. They spontaneously experiment and experience and re-experience again. They select, combine, and test, seeking to find order in their experiences - "which is the mostest? which is the leastest?" They smell, taste, bite, and touch-test for hardness, softness, springiness, roughness, smoothness, coldness, warmness: they heft, shake, punch, squeeze, push, crush, rub, and try to pull things apart.

-Richard Buckminster Fuller

In this chapter, the specific selection criteria to choose events for the muon analysis is described, both in selecting the neutrino events at the ND for the extrapolation and in selecting the muon sample at the FD. For use by MINOSians, the reconstructed variable name in the standard minossoft R1.24 Physics Analysis Ntuple (PAN) files as generated by the minossoft code in Mad/MadDpAnalysis.cxx is indicated in math font. Along with the variable name and the appropriate unit of measure, the unitless cut value as implemented in this analysis code is indicated. These PAN variable names can change between software releases but have remained fairly stable over the last $\sim$ seven releases. Consult the \$SRT_PUBLIC_CONTEXT/Mad/ software package in the appropriate minossoft release for current variable names and definitions.

The goal of the non-fiducial analysis is to select beam neutrino-induced CC events and to measure the momentum and direction of the muon. To achieve this end, three event selections at the FD were studied in this work. The first was developed to maximize sensitivity to the oscillation parameters when applied to Monte Carlo mock data trials and is assumed to be used throughout this work unless otherwise specified. This selection 
includes both muons from interactions in the rock as well as in the non-fiducial region of the detector. It is desirable to reclaim these "in-between" events that are not selected as rock muons and are not selected in the main fiducial $\mathrm{CC}$ analysis, as they provide another handle for determining the oscillation parameters. The second is included to select a sample pure in rock muons while attempting to eliminate contained vertex events, mainly to compare event rates between data and MC. The third relies on human judgment to assign an event interaction type (NC, CC), flavor $\left(\nu_{e}, \nu_{\mu}\right)$, and category (rock muon, contained vertex, cosmic muon, etc.) to each event recorded in the beam spill window. The author's scan decisions are compared with the first two selections in Appendix D.

In all contexts, by "fiducial analysis" or "main CC analysis" the author refers to the first MINOS beam-neutrino analysis published[1] in Physical Review Letters in 2006. This analysis used CC-like events selected with a track vertex inside of the fiducial volume of both detectors. At the time of this writing, an updated fiducial analysis is being prepared that will utilize a data sample of $2.50 \times 10^{20}$ POT, nearly doubling the 2006 sample of $1.27 \times 10^{20}$ POT. In these discussions, the PAN variable name $q p$ refers to the quantity $q / p$ (not $q \times p$, or the reconstructed inverse track momentum multiplied by the particle charge.

\subsection{Near Detector selection}

At the Near Detector, the goal of this analysis is to determine the energy spectrum of charged current $\nu_{\mu}$ interactions, to be used in the extrapolation to the Far Detector (see Chapter 5 for description of the extrapolation method used). Due to the stability of the beam spectrum over the first year of running and the high event rate at the ND, the data considered here corresponds to roughly every third sub-run taken between June 2005 and February 2006. This sample constitutes $0.48 \times 10^{20}$ Protons on Target. Only "spill" files are used in this selection, in which every snarl was accompanied by a spill signal indicating that the beam was on. Event selection cuts[59] equivalent to those used in the fiducial analysis are used as follows:

- The beam must be within normal running parameters; pass_beamcuts $==1$ :

- The snarl time must fall within $\pm 1.0 \mathrm{~s}$ of the nearest beam spill time; $\mid$ snarlt timedb $\mid<1.0$.

- The horn current must fall between -200 and -155 kA (standard running is 
$-185 \mathrm{kA}$, with negative current focusing positively charged hadrons that decay to $\left.\mu^{+}+\nu_{\mu}\right) ;-155>$ horndb $>-200$.

- The number of protons on target (POT) must be between $0.5 \times 10^{12}$ and $50.0 \times 10^{12}$ POT; $0.5<\operatorname{pot} d b<50.0$.

- The horizontal position of the beam on the target must fall between -2.0 and $-0.01 \mathrm{~mm} ;-0.00001>$ hhposdb $>-0.002$.

- The vertical position of the beam on the target must fall between 0.01 and $2.0 \mathrm{~mm} ; 0.00001<$ vvposdb $<0.002$.

- The beam width must fall between 0.1 and $1.5 \mathrm{~mm}$ in the horizontal direction, 0.1 and $2.0 \mathrm{~mm}$ in the vertical direction; $(0.0001<$ hhwidthdb $<$ $0.0015) \& \&(0.0001<$ vvwidthdb $<0.005)$.

The beam center is located away from the coordinate origin, hence the asymmetric position requirements.

- At least one reconstructed track must be present in the snarl; ntrack $>0$.

- The track must have a reconstructed vertex within the fiducial volume, defined as a right circular cylinder of radius $1.0 \mathrm{~m}$ and length $4.0 \mathrm{~m}$, with its axis along the beam direction; $(1.0<\operatorname{trkvtx} z<5.0) \& \&\left(\operatorname{sqrt}\left((\operatorname{trkvtx} x-1.4885)^{2}+(\right.\right.$ trkvtxy $\left.0.1397)^{2}\right)<1.0$ ).

- The track must have "good quality", either passing the track fitter quality check or having a minimally consistent set of beginning and end points in both the $u$ and $v$ views; $($ trkfitpass $==1) \|((\mid$ trkplbu - trkplbv $\mid \leq 5) \& \&(\mid$ trkpleu - trkplev $\mid \leq$ 40) \&\& (trkpleu $<270 \& \&$ trkplev $<270))$.

- The track must have a negative or zero reconstructed charge (those events with momentum measured from range due to incomplete curvature information are assigned a charge of zero), consistent with a $\mu^{-}$arising from a $\nu_{\mu}$ charged current interaction; trkqp $\leq 0$.

- The likelihood Particle IDentification (PID) must be greater than -0.1 to remove NC-like events. See Section 4.3 for an explanation of the PID construction and performance; pid $0>-0.1$. 


\subsection{Far Detector selection}

The data considered here was taken between Far Detector Run 31720 (20 May 2005) and Run 33791 (25 February 2006) and constitute $1.27 \times 10^{20}$ Protons on Target. Due to the desire to study a totally independent set of events from that of the fiducial analysis[1], the main selection cut used in this analysis is to require that the event vertex fall outside of the FD fiducial volume. In each of the following selections, care is taken to avoid any overlap in events between this analysis and that of the main $\mathrm{CC}$ analysis. There were also short periods of time in which the Far Detector was not fully operational, due to high voltage trips, magnetic coil cooling problems, or entire electronics rack failures. The GPS time stamp of each event was cross-checked with known problem times (a bad run/subrun list) to ensure that the detector was healthy when the event was recorded. As seen in Table 4.1 , only $0.9 \%$ of pre-selected events are removed due to problems with the detector.

\subsubsection{Inverse fiducial volume cuts}

The inverse fiducial event selection is designed to include all rock vertex (RV) events as well as those events that have a contained interaction vertex in the outer regions of the detector. These contained vertex (CV) events are selected in such a way as to permit no overlap with the events selected by the main $\mathrm{CC}$ analysis, thus providing an independent determination of the oscillation parameters. In all contexts, by "inverse fiducial", "antifiducial", or "non-fiducial" the author refers to that volume which falls outside of the standard FD fiducial volume, defined as follows. One or more of these criteria qualify an event as "antifiducial":

- The track vertex is reconstructed at a squared radius greater than $14.0 \mathrm{~m}^{2}$; trkvt $x x^{2}+t r k v t x y^{2}>14.0$.

- The track vertex is reconstructed in the front $50 \mathrm{~cm}$ of the detector; trkvtxz $<0.5$.

- The track vertex is reconstructed in the $\sim 2 \mathrm{~m}$ region defining the Super Module gap and the volume surrounding it; $14.3<$ trkvtx $z<16.2$.

For these events that fall outside of the fiducial volume, the following selection cuts are used: 
- The beam must be within normal running parameters (see Section 4.1 for this definition); pass_beamcuts $==1$.

- There must be at least one reconstructed track; ntrack $>0$.

- There can be no Light Injection (LI) activity present in the snarl (see Section 2.4; litime $=-1$.

- The event raw pulse height must remain below one million ADC counts (this removes LI activity that fails to obtain an litime tag, as only a small fraction of a percent of beam events register a pulse height this high); litag $<3$ or equivalently allph $\leq 1 e 6$. See the discussion below for justification of the cut placement $<3$ instead of $==0$.

- The track vertex position must lie in the first $28 \mathrm{~m}$ of the detector (due to poor momentum resolution in the rear $2 \mathrm{~m}$ of the detector - see Section 6.6.5); trkvtxz< 28.0 .

- The track must have a negative reconstructed charge or no reconstructed charge (if curvature was suspect and momentum was assigned by range); $\operatorname{trkqp} \leq 0$.

- The event with the largest total pulse height in a snarl is used. At the Far Detector, there are not many snarls with multiple events. Such instances almost always indicate a reconstruction problem where a single event was cut into multiple events.

- The likelihood Particle IDentification (PID) parameter must be greater than -0.5 to remove NC-like events. This cut value is looser than the value at the ND due to the filtering effect of the rock on hadron activity. Since the non-fiducial event sample has an intrinsically higher charged current purity, this cut is relaxed at the FD. See Section 4.3 for an explanation of the PID construction and performance; pid0 $>-0.5$.

- The "data quality cut" is imposed, which identifies runs that are thrown out of physics analysis due to detector abnormalities such as High Voltage trips, incapacitated electronics racks, spurious detector activity, etc; pass_fd_qualcuts $==1$

Due to the nature of the events detected outside of the fiducial volume, the selection must be optimized for such events, which have characteristically lower shower activity 
and a greater uncertainty in the magnetic field strength. The FD selection cuts that are different than the fiducial analysis cuts are as follows:

- There was development of a selection cut that removed an event if it began in the first two planes and ended in the last two planes that were fed to a single electronics crate. This was motivated by the fact that LI is activated in one-crate blocks, and if the litime variable failed to register this activity, such events could be removed with this cut. litag is incremented by one for each occurrence of this reconstruction pattern. Two selected events in this sample would have been removed by this cut. A bias present in the non-fiducial sample is that many rock events begin in the first two planes of either Super Module, as these two "faces" have the largest exposed area of any group of two planes in the detector. Such events automatically satisfy half of the criteria for this cut. Since there are no apparent LI events (verified by hand-scanning) in the selected sample, this cut is not used in this analysis. litag $<3$ instead of litag $==0$.

- The cosine of the reconstructed track vertex direction along the beam axis must fulfill $\cos \theta_{\text {beam }}>0.5\left(\theta_{\text {beam }}<60^{\circ}\right)$. This cut is looser than the 0.6 requirement for the main analysis in order to allow for muons that have multiple scattered inside of the rock before reaching the detector. Loosening this cut further allows for an unacceptable background rate from cosmic muons; reco_dircosneu $>0.5$. For rock and non-fiducial muon events modeled in GMINOS, the expected contribution of events with reco_dircosneu $<0.5$ is less than $0.1 \%$. See Figure 4.1 for a display of the exponential falloff in event rate with decreasing reco_dircosneu.

- For tracks that exit the detector, the momentum is measured by curvature. A track is considered as exiting the detector if the reconstructed track endpoint falls within the region outside of the fiducial volume. Such events are only selected if $|(q / p) / \sigma(q / p)|>3.0$, that is, the estimated uncertainty in the curvature is no more than $33.3 \%$ of the measured curvature. All tracks that do not exit the detector (meaning their endpoint was reconstructed inside of the fiducial volume) are considered as stopped inside and the momentum is measured by range. This criteria is motivated by the desire to have a topological containment requirement that does not rely explicitly on the knowledge of the magnetic field. If containment is instead assigned by some comparison of the momentum from range and from curvature (i.e. requiring the curvature measurement to be some percentage higher 


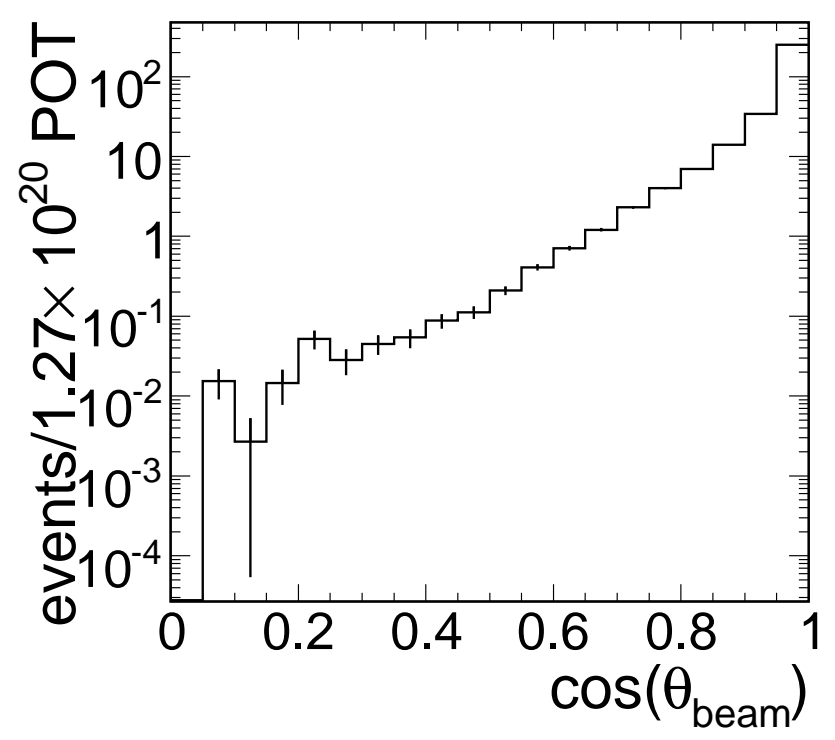

Figure 4.1: Angular distribution of antifiducial muon events in GMINOS.

than the range measurement), significant differences between the data and Monte Carlo are observed. As seen in Table 4.1, the fraction of data events with momentum assigned by range by the topological requirement is $0.86 \pm 0.09$, consistent with the MC expectation of 0.83 . This statistic is also noted for the high percentage of events that have momentum assigned from range (which has been assigned a $2 \%$ systematic uncertainty) instead of from curvature (which has a higher systematic uncertainty, $\sim 5 \%)$; if $\left(\left(\right.\right.$ trkend $x^{2}+$ trkendy $^{2}>14.0 \|$ trkendz $<0.5 \| 14.3<$ trkendz $<16.2 \|$ trkend $z>28.0)$ \&\& $\mid$ trkqp $\mid /$ trkeqp $>3.0)$ assign $\mid 1 . /$ trkqp $\mid$ as the measured momentum.

- Only events with reconstructed momentum up to $10 \mathrm{GeV} / \mathrm{c}$ are included in the oscillation fit. This choice was made by binning the expected distribution for 10.0e20 POT (the expected exposure at the end of the experiment) in $1 \mathrm{GeV}$ bins and looking for the bin where the expected deficit due to oscillations (defined by the PRL best-fit values of the parameters) dropped below one event. Furthermore, uncertainty in the neutrino flux arising from $K^{ \pm}$decay in the beam line generates 
a larger fractional uncertainty in the rate of muon events above this energy range.

- The cut on event arrival time is tighter than that in the fiducial analysis due to the larger cosmic muon background in the non-fiducial volume. The relevant timing variable is the difference of the measured arrival time and the expected arrival time of the first neutrino from each spill, plus the time from the beginning of the snarl to the time of the earliest hit in the event. This quantity is required to be within the 10 $\mu$ s beam spill window; $0.0 \times 10^{-6}<($ snarlt - fartdb + evttimemin $)<10.0 \times 10^{-6}$.

Some care was taken in deciding to exclude the positive charge sign tracks from this analysis (tracks with no assigned charge are retained if their endpoint is contained and a momentum measurement is made by the range in the detector). Since the $\bar{\nu}_{\mu}$ flux peaks at a higher energy than the $\nu_{\mu}$ flux, the expected deficit due to oscillation in this sample is small. The true rock $\mu^{+}$flux is $\sim 10 \%$ of the total event rate but we reconstruct $\sim 15 \%$ of events with $q p>0$. The true $\mu^{-}$tracks with misreconstructed charge generally do not have a good momentum measurement from curvature, but if they have a contained endpoint the range measurement is accurate and this small number of events will enhance the expected oscillation signal. A study revealed that including the $q p>0$ sample only improves the $\chi^{2}$ discrimination (at $1.27 \mathrm{e} 20$ POT) by 0.08 (considering the statistical improvement only). With greater beam exposure and better understanding of the $\bar{\nu}_{\mu}$ flux in the future, it will be worthwhile to include these $\mu^{+}$events in the analysis.

\subsubsection{Rock muon cuts}

As a subset of the events selected by the above cuts, a sample of events almost entirely composed of rock muons can be selected. The reconstructed track vertex best distinguishes muons entering from the rock and muons created by neutrino interactions in the detector. Specifically, implementing a cut on the transverse distance to the detector edge (for events entering the sides of the detector) or a cut on the plane number in which the track begins (for events entering the front or Super Module gap). The rock muon selection cuts require one of the following:

- The track vertex lies within $10 \mathrm{~cm}$ of one of eight detector side faces; $|\operatorname{trkvtxX}|>$ 3.9 , where $X$ is one of $x, y, u, v$.

- Track vertex lies in the first two planes of SM 1; trkplb $<3$.

- Track vertex lies in the first two planes of SM $2 ; 249<t r k p l b<252$. 
Table 4.2 displays the same selection cuts as in Table 4.1 but with the Rock Vertex $(\mathrm{RV})$ and Contained Vertex (CV) Monte Carlo samples separated (these columns are added together to obtain "MC Exp." in Table 4.1). The active volume used in selecting rock muon events is a small fraction of the non-fiducial volume. Since the neutrino flux is uniform at the FD, the fraction of $\mathrm{CV}$ events labeled as rock events can be used as a measure of this fraction. Table 4.2 shows that $\sim 27 \%$ of the active volume used in this analysis is serving as the active volume for rock event selection. The rock selection properly labels $99.1 \%$ of Monte Carlo events generated in the rock.

\subsubsection{Hand scanning}

While only the author's scan decisions are indicated in Appendix D, several others participated in the scanning effort. M. Goodman identified questionable events in the data in order to search for reconstruction pathologies that might not be modeled in the Monte Carlo. This was accompanied by the author's focused scan of Monte Carlo events to ensure that events were being reconstructed similarly between data and MC. We found no inconsistencies. The author was fortunate to mentor two high school students from the Illinois Math and Science Academy, Amy Allen and Sarah Schwarzentraub. They were both able, with modest training, to distinguish the important features of muon tracks in the MINOS Far Detector and chose similar classifications to those of the author and the senior scientists involved in the scanning effort.

More extensive work in scanning Far Detector data and Monte Carlo has been performed by M. L. Marshak and W. A. Mann[60],[61] in preparation of a measurement of the oscillation parameters using event selection entirely by hand. The main focus of this scanning analysis is to accurately resolve the low-energy rise (characteristic of an oscillation signal, as opposed to some other disappearance mechanism) in the neutrino energy spectrum for contained vertex CC events, where the main CC analysis has relatively poor selection efficiency.

The author's scan of the spill data stream was completed by March 2006, shortly after the cutoff for the $1.27 \times 10^{20}$ POT data set. The event selection criteria for the primary cut-based analysis was not fully developed at this time, and the relevant RV and CV Monte Carlo samples had not yet been combined. As a result, the author was uninformed about the expected number of events during the data scanning. The MC was used at this point to train the author's scan selection.

For all event scanning in this work, the Mad/macros/EvDisplay.C macro was used, 
available in the standard minossoft installation. In the author's scan, a clean muon-like track exiting the reconstructed vertex region was used to identify charged current events. As a further classification, such events were deemed as rock muons if they satisfied one of two criteria:

- The track vertex was reconstructed within $10 \mathrm{~cm}$ of any of the eight side faces of the detector.

- The track vertex was reconstructed in the first two planes of SM 1 (the front face of the detector) or the first two planes of SM 2 (exposed by the SM gap).

Further scanning studies were completed for a variety of reasons. The most important of these studies was done to ensure that the data events were being reconstructed in a consistent way with the Monte Carlo events. Of the 232 selected events for this analysis, one was not classified as either charged current or neutral current due to reconstruction problems. A display of this event is shown in Figure 4.2. Such events are uncommon, but not absent from the Monte Carlo sample. A similar fraction of ambiguous NC/CC events was found in the Monte Carlo as was found in the selected data.

Another purpose of scanning was to ensure that NC-like events in the data were represented at the same rate in Monte Carlo. One class of events that appears NClike (more negative PID value) results from a neutrino interaction in the rock near the interior cavern surface. Both neutral current and charged current events can spray a hadron shower into the detector. In the latter case, the accompanying muon does not always intersect the detector, making a true CC event appear NC-like. This ambiguity can be seen in Table D.1, in which the author's scan decision labeled nine events as neutral current, while only 4.1 true NC events are expected from the Monte Carlo. The slight excess of NC-like events in the selected data sample is likely due to CC events in which the muon does not intersect the detector. Such events are observed in the Monte Carlo at approximately the same rate as the NC rock events, having a pion or proton track that mimics a low-energy muon track. Three events in the selected data sample appeared to be possible hadron spray from a rock vertex interaction. One such event is shown in Figure 4.3, where a group of hits begins spread out in the first plane and only penetrates $\sim$ one meter into the detector. 

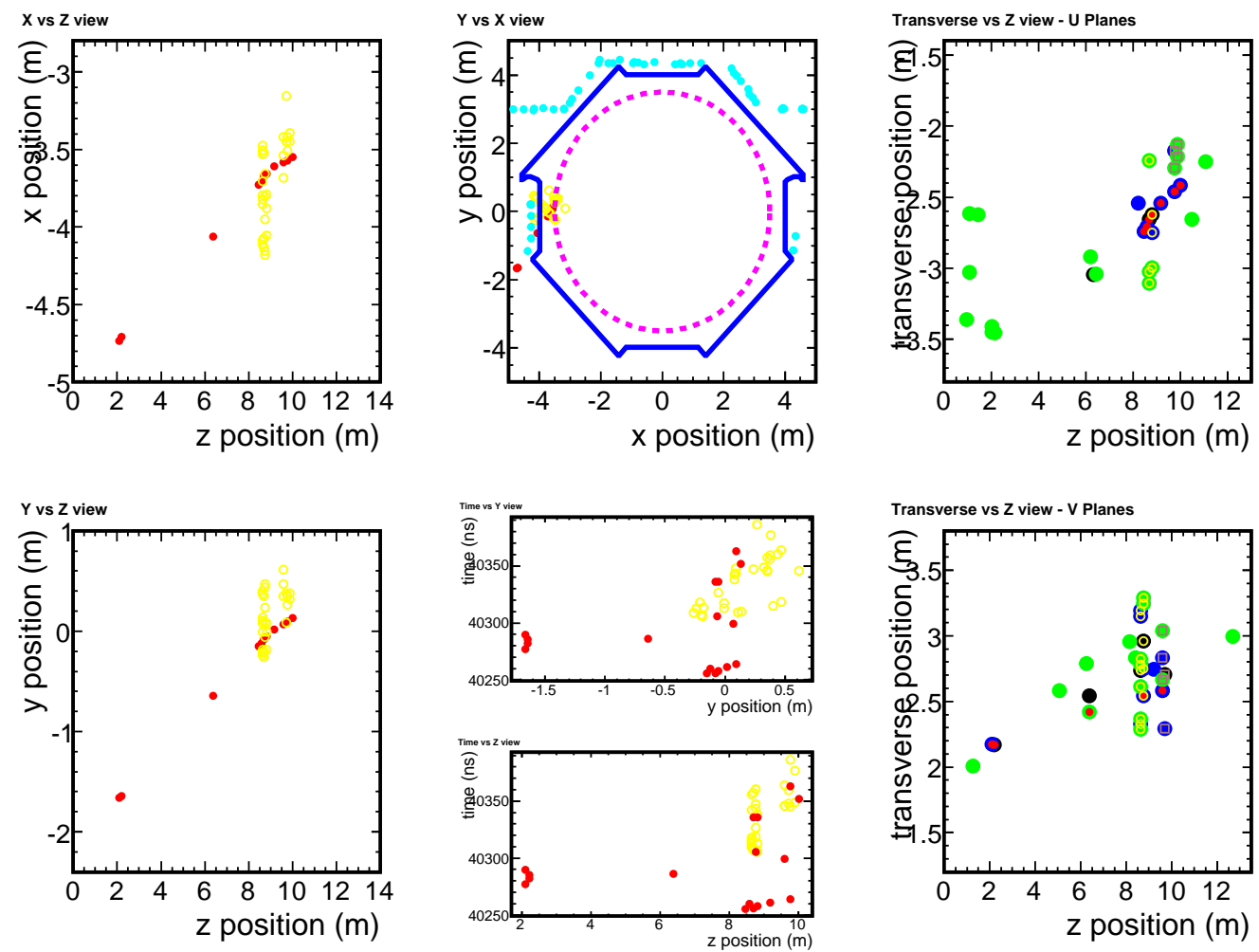

Figure 4.2: Selected event with reconstruction problems: Run 32569, Snarl 91668.

\subsection{Particle ID construction}

A likelihood parameter that identifies an event as CC-like or NC-like is desirable in removing as much neutral current background as possible. D. Petyt has developed[62] such a parameter (named PID, for Particle IDentification) for use in the MINOS fiducial CC analysis, closely following the likelihood construction of the Super-Kamiokande atmospheric neutrino analysis. This method was adopted and modified to use the event characteristics for interactions in the antifiducial volume.

Three variables enter into the PID calculation: the fraction of total event pulse height carried by the track trkphfrac (related to the y value of the neutrino interaction), the average pulse height per plane attributed to the reconstructed track trkphplane (related to the energy loss characteristics of the track particle), and the total number 

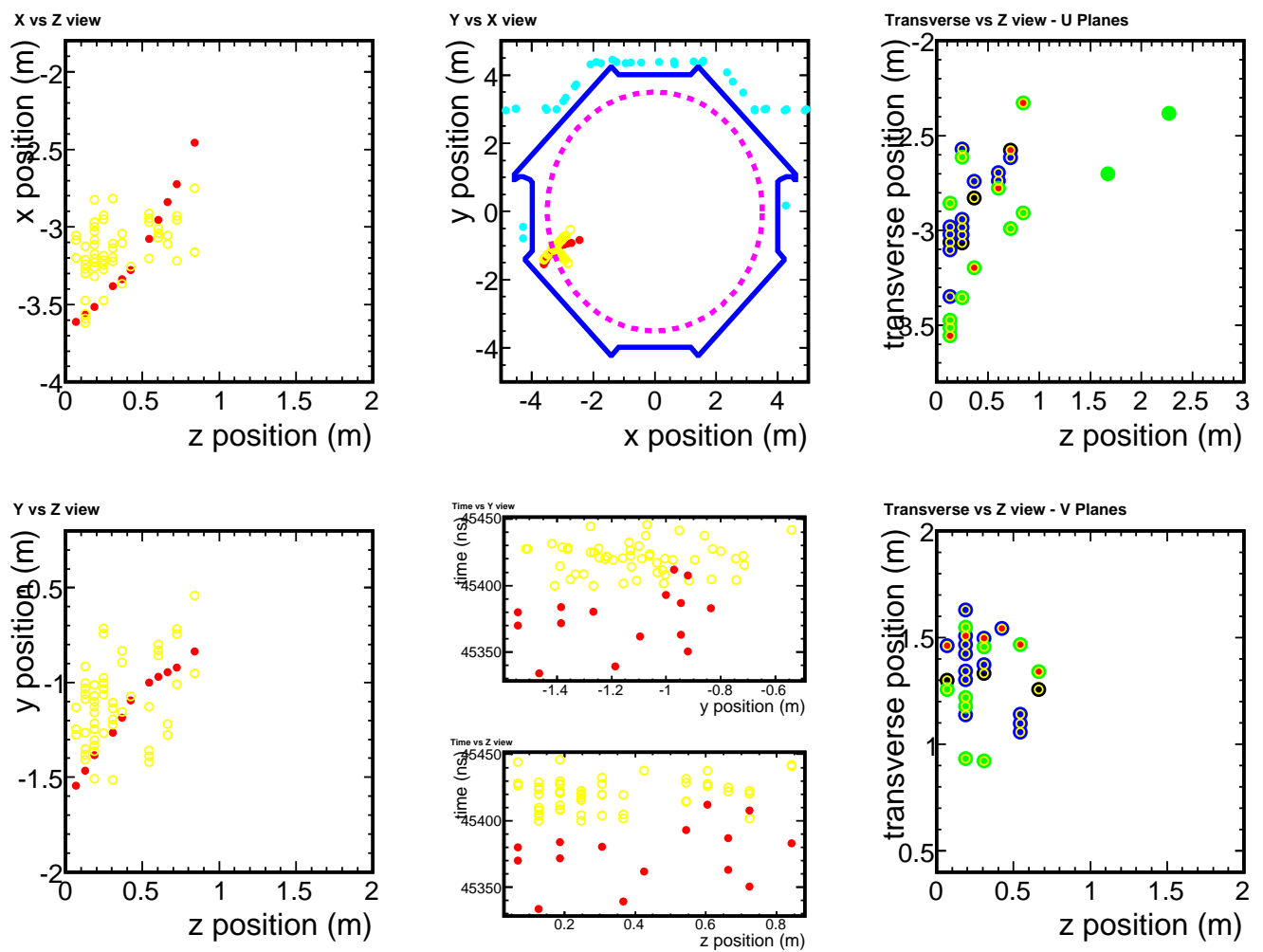

Figure 4.3: Possible hadron shower event from neutrino interaction near front of cavern wall: Run 33184, Snarl 235339.

of planes intervening between the reconstructed event vertex and endpoint, evtlength (related to the momentum of the track particle). Each of these distributions demonstrate considerable shape differences between true NC and CC events as modeled in the Monte Carlo. In the following plots, all selection cuts (described in Section 4.2.1) are used with the exception of the track momentum cut (to demonstrate the full momentum spectrum) and the PID cut (to demonstrate the full distribution of PID values).

Figures 4.4 - 4.6 display these three reconstructed variables that enter into the PID calculation. In each figure, the Rock Vertex (RV) events are on the left, the Contained Vertex $(\mathrm{CV})$ events are in the middle, and the combined $(\mathrm{RV}+\mathrm{CV})$ are on the right. In all plots, both $\mathrm{NC}$ and $\mathrm{CC}$ distributions are normalized to 1.0 to provide the true Probability Density Function (PDF) used as input to the PID calculation. The relative 
ratio between the actual number of $\mathrm{CC}$ and NC events is approximately 200:3.
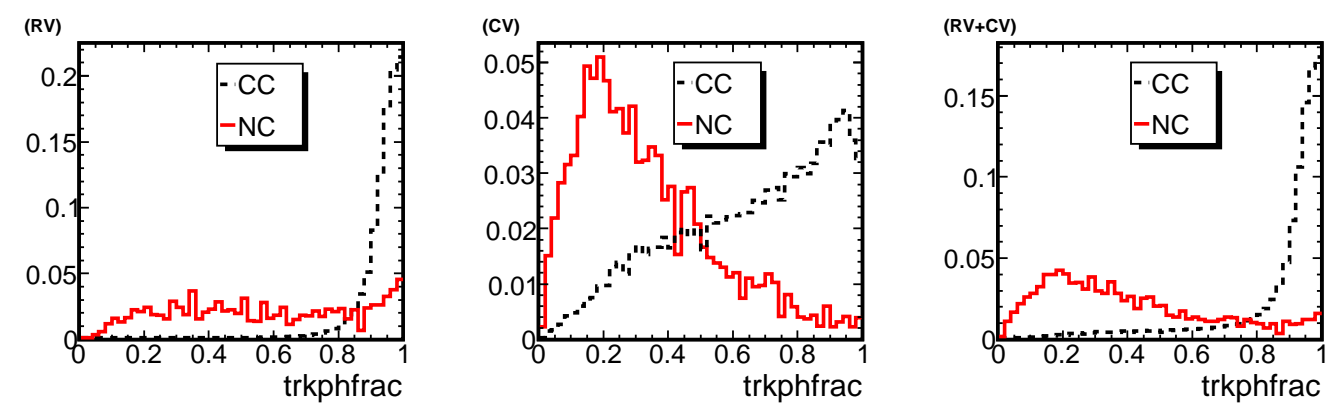

Figure 4.4: Fraction of total event pulse height contained in reconstructed track. From left to right: Rock Vertex (RV), Contained Vertex (CV), and total events (RV+CV). Charged current distribution in black dashed line; Neutral current distribution in solid red line. All distributions are normalized to 1.0.

The three distributions are then combined in the following way to obtain one PID value for each reconstructed event:

- For each of the three variables $v$, the reconstructed quantity is used to obtain the value of the Probability Density Function for both true NC events $\left(P_{N C}^{v}\right)$ and true $\mathrm{CC}$ events $\left(P_{C C}^{v}\right)$. The boundaries $0 \leq P \leq 1$ must hold for any value of the PDF as a function of the variable of interest.

- The likelihood of each interaction is defined to be

$$
P_{N C}=\prod_{v=1}^{3} P_{N C}^{v} \text { and } P_{C C}=\prod_{v=1}^{3} P_{C C}^{v} \text {. }
$$

- The negative $\log$ likelihood is formed as $L_{N C}=-\ln P_{N C}$ and $L_{C C}=-\ln P_{C C}$

- The PID parameter is defined to be $P I D=\sqrt{L_{N C}}-\sqrt{L_{C C}}$

The resulting PID distributions are shown in Figure 4.7. Nominally, a PID value of zero would represent an event with an equal likelihood of being a true NC or CC event. One can get a sense for the behavior of this function with the following reasoning: an event that has CC-like characteristics will have a large $(\sim 1)$ value of $P_{C C}$ and a small $(\sim 0)$ value of $P_{N C}$. The negative natural logarithm of a number close to one/zero is a 

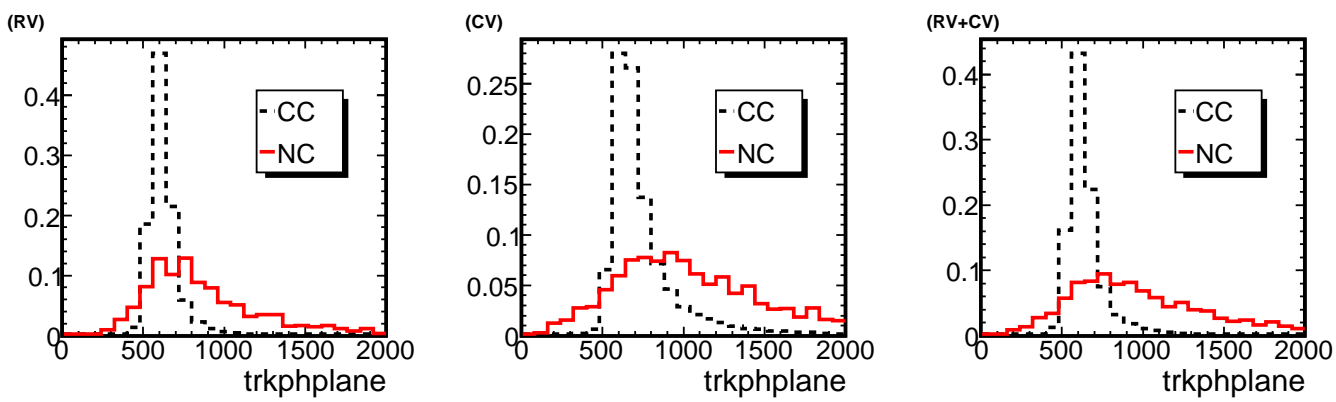

Figure 4.5: Average pulse height per plane for reconstructed track. From left to right: Rock Vertex (RV), Contained Vertex (CV), and total events (RV+CV). Charged current distribution in black dashed line; Neutral current distribution in solid red line. All distributions are normalized to 1.0.

small/large positive number. The square roots preserve the relative size of $L_{N C}$ (larger) and $L_{C C}$ (smaller), making $P I D>0$ for this CC-like event.

Optimizing the sensitivity of this analysis (i.e., minimizing the area enclosed by the confidence level contours in oscillation parameter space when the fit is performed) by scanning over different values of the PID cut leads to the conclusion that one should discard all events with pid0 $<-0.5$. The PDFs shown in Figure 4.7 must be taken with the relative $\mathrm{CC}: \mathrm{NC}$ rates in mind. For example, the apparently large contribution of $\mathrm{NC}$ background events above pid0 $=-0.5$ in the $\mathrm{RV}+\mathrm{CV}$ plot integrates out to an expectation of only 4.1 events, or $1.6 \%$ of the total selected sample.

\subsection{Efficiency and purity of event selection}

The standard antifiducial event selection has a demonstrable efficiency in selecting rock and non-fiducial CC muon events and a well-defined CC purity, both assessed by applying the selection to Monte Carlo events and categorizing both selected and rejected events based on their truth interaction type and neutrino flavor. The cumulative effect of the event selection on Monte Carlo events (containing both NC and CC interactions of all neutrino flavors) is displayed in Tables 4.1 and 4.2. 

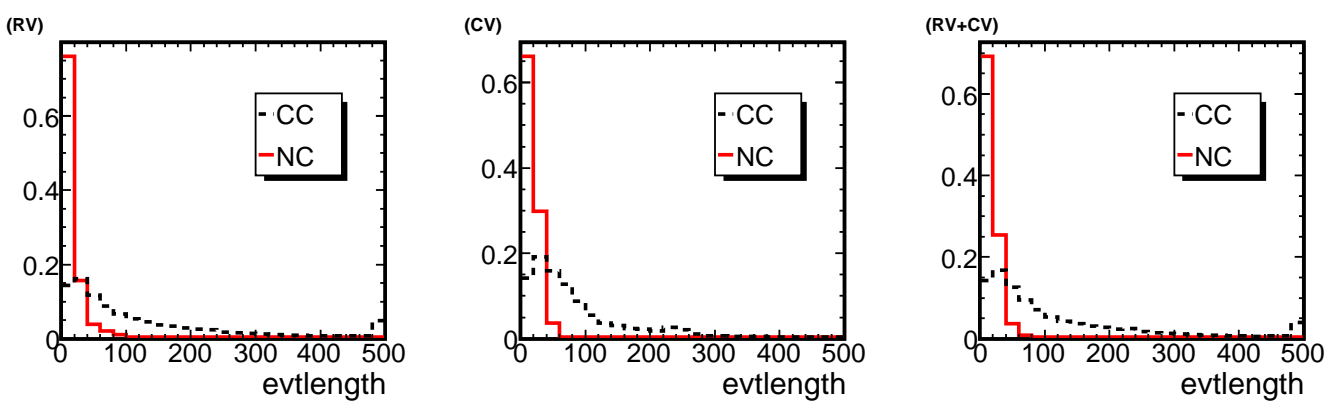

Figure 4.6: Event length in number of planes. From left to right: Rock Vertex (RV), Contained Vertex $(\mathrm{CV})$, and total events $(\mathrm{RV}+\mathrm{CV})$. Charged current distribution in black dashed line; Neutral current distribution in solid red line. All distributions are normalized to 1.0 .

The definitions of efficiency and purity are:

$$
\text { efficiency }=\frac{\text { true } \nu_{\mu} C C \text { events after selection }}{\text { true } \nu_{\mu} C C \text { events before selection }}
$$

and

$$
\text { purity }=\frac{\text { true } \nu_{\mu} C C \text { events after selection }}{\text { all events after selection }} .
$$

The numerator in both cases is the number of events passing all selection criteria in MC that have a true charged current interaction of a $\nu_{\mu}$. Here the reconstructed momentum cut is omitted (which is used only in obtaining the oscillation parameter fit) in order to display the performance for a wide range of momenta. The label before selection indicates that an event had the following "preselection" requirements: at least one track must be reconstructed with its vertex outside of the fiducial volume, and we must have a viable measurement of the track momentum (either by range or curvature, see Section 4.2.1 for details). The label all events indicates an event sample that includes all events passing the selection criteria, including neutral current and charged current interactions of all neutrino flavors.

Figures 4.8 - 4.11 display the efficiency and purity as calculated above. Each is displayed as a function of reconstructed muon momentum (up to $30 \mathrm{GeV} / \mathrm{c}$ ) as well as a function of true neutrino energy (up to $60 \mathrm{GeV}$ ). The plots indicate a bin-by-bin efficiency or purity and do not show statistical uncertainty bars, as these plots become 

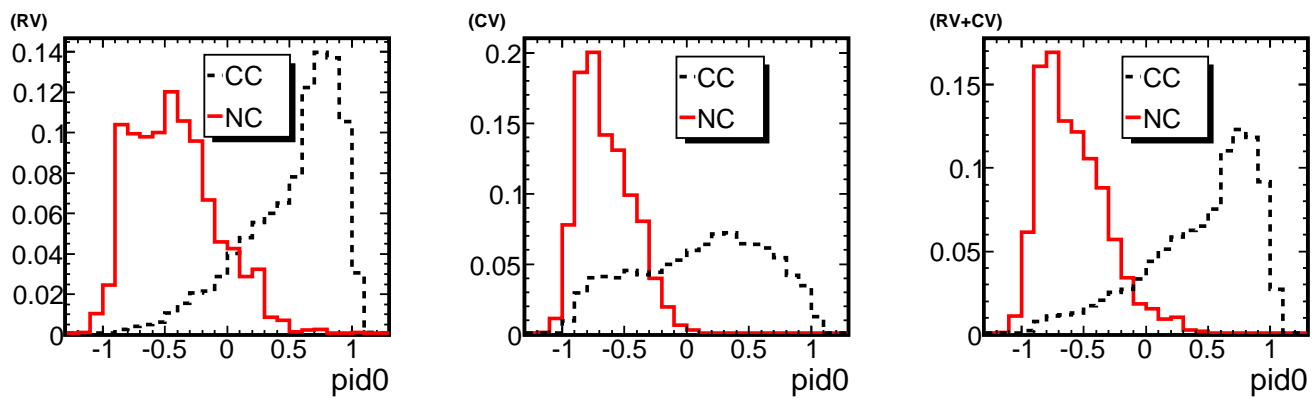

Figure 4.7: Particle ID parameter as calculated in Section 4.3. Charged current distribution shown as black dashed line; Neutral current distribution shown as solid red line. All distributions are normalized to 1.0.

too busy. See Figures 5.10 and 5.8 for a sense of the decreasing statistics at high $P_{\mu}$ and $E_{\nu}$.
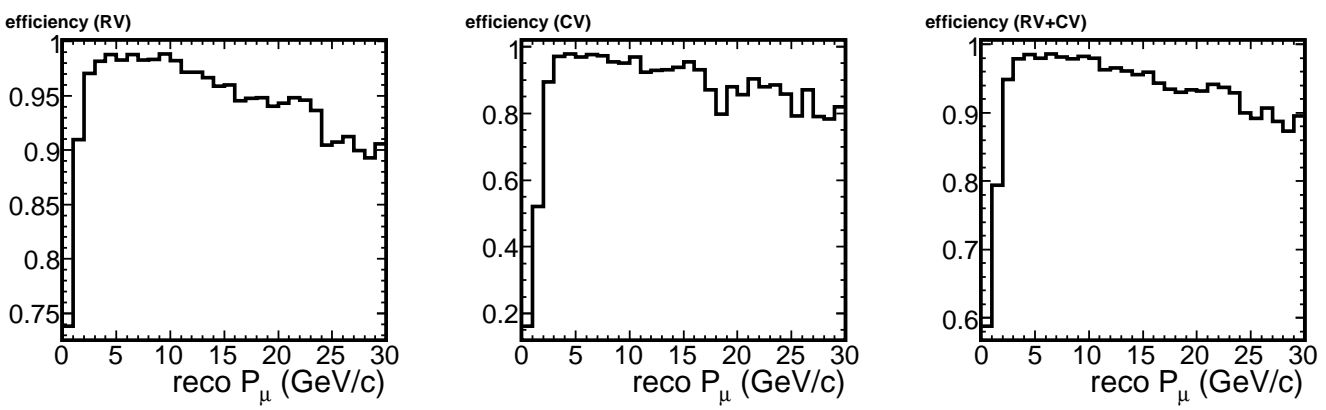

Figure 4.8: Charged current selection efficiency as a function of reconstructed muon momentum. Average values: 94.3\% (Rock Vertex), 86.3\% (Contained Vertex), 92.8\% (Combined). Note the different vertical scales.

Some trends should be noted. The decreasing efficiency with reconstructed momentum in Figure 4.8 is entirely due to the transverse momentum cut, without which these efficiency plots are flat at high momentum. Keep in mind the number of events present at these high reconstructed momenta are few. The low efficiency at low energy in the $\mathrm{CV}$ plot in Figure 4.9 is due to the PID cut removing true CC events with a short muon 

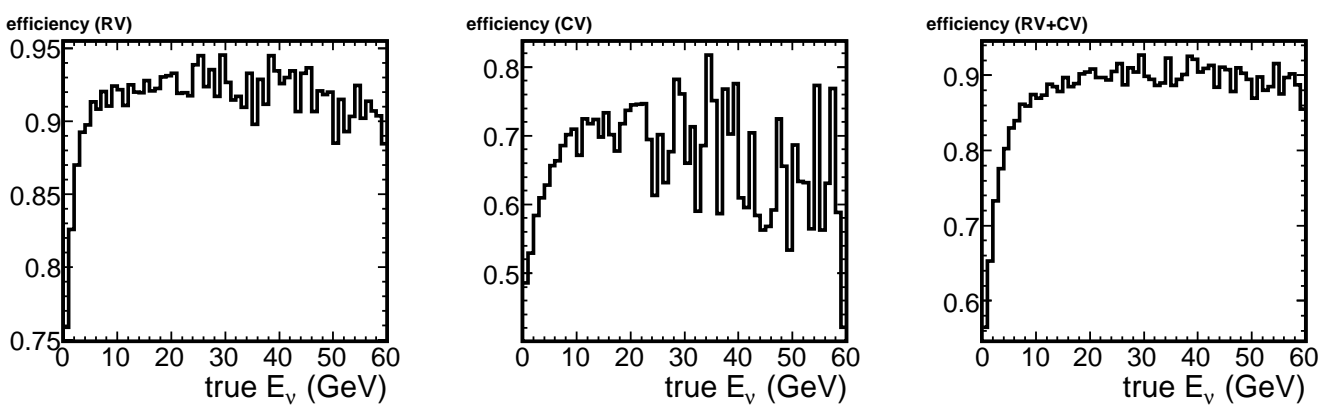

Figure 4.9: Charged current selection efficiency as a function of true neutrino energy. Average values: 91.4\% (Rock Vertex), 66.5\% (Contained Vertex), 87.7\% (Combined). Note the different vertical scales.

track.

The $\nu_{\mu}$ charged-current purity of the RV sample in Figure4.10 is nearly complete, with the lowest momentum bins corresponding to neutral-current events with a reconstructed pion or proton. The same class of events causes the low-momentum dip in the CV plot. The dip in the RV purity plot in Figure 4.11 corresponds to the $\bar{\nu}_{\mu}$ events that are improperly reconstructed with a negative (or zero) charge, and its shape bears a striking resemblance to the shape of the antineutrino spectrum. In the CV purity plot in the same figure, the slight dip at low energy is due to the neutral-current events that pass selection.

\subsection{Backgrounds}

Three main backgrounds were studied that could mimic a true muon coming from a NuMI beam interaction: cosmic ray muons (which are produced by the interaction of primary cosmic rays in the upper atmosphere), neutral current interactions (which may have a charged proton or pion as a reconstructed track), and $\tau$ appearance due to $\nu_{\mu} \rightarrow \nu_{\tau}$ oscillation (the decay of a $\tau$ leading, in some cases, to a muon that is detected).

\subsubsection{Cosmic ray muons}

Because this analysis selects events with a reconstructed vertex outside of the fiducial volume, much care must be taken to eliminate the cosmic ray muon background. Three 

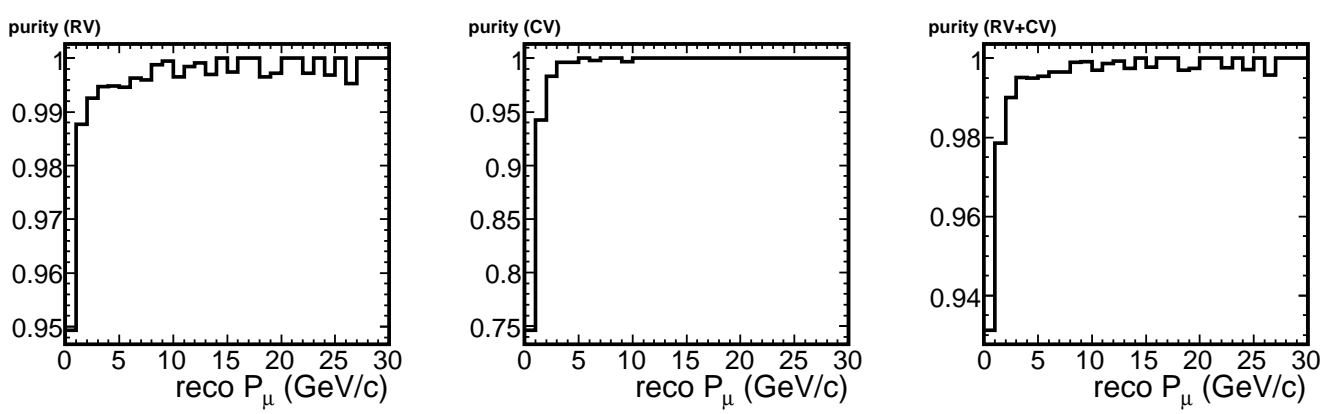

Figure 4.10: Charged current selection purity as a function of reconstructed muon momentum. Average values: 99.6\% (Rock Vertex), 98.9\% (Contained Vertex), 99.5\% (Combined). Note the different vertical scales.

cuts provide the bulk of the background rejection: the track direction cut, the arrival time cut, and the transverse momentum cut. The track direction cut requires a track to originate within $60^{\circ}$ of the beam direction, and passes $13 \%$ of cosmic ray muon tracks that have a good momentum measurement. The timing cut removes all cosmic ray muons that fall outside of the expected arrival time of the $10 \mu$ s beam spill window, passing $8 \%$ of those events that fall within the $\sim 130 \mu$ s spill-triggered readout window.

Part of the regular MINOS run control in data acquisition is to obtain detector response in a simulated spill window (a "fake spill") between regular beam spills. A sample of $2.65 \times 10^{6}$ fake spills was used initially to estimate the background for this analysis. This corresponds to roughly $40 \%$ of the detector livetime during beam spills in this analysis. Most cosmic muons have a large momentum component transverse to the beam direction, as they approach the detector from the near-vertical direction. A cut requiring events to have $P_{\text {trans }}<2.5 \mathrm{GeV} / \mathrm{c}$ effectively removes the cosmic ray background while retaining $~ 99 \%$ of beam Monte Carlo events. Zero events were selected in the fake spill sample, leading to a $90 \%$ confidence level upper limit on the number of cosmic muons in the true spill data of 5.9 events.

Because the live time of the fake spill sample used was only $40 \%$ of the data livetime (resulting in a loose upper limit), a further study was undertaken to precisely determine the expected background rate in the selected data sample. A cosmic ray sample was used, taken from the "all" event data stream (not requiring a spill trigger) during a span of 5.2 live days during February 2006. As seen in Table 4.1, the precision to which the 

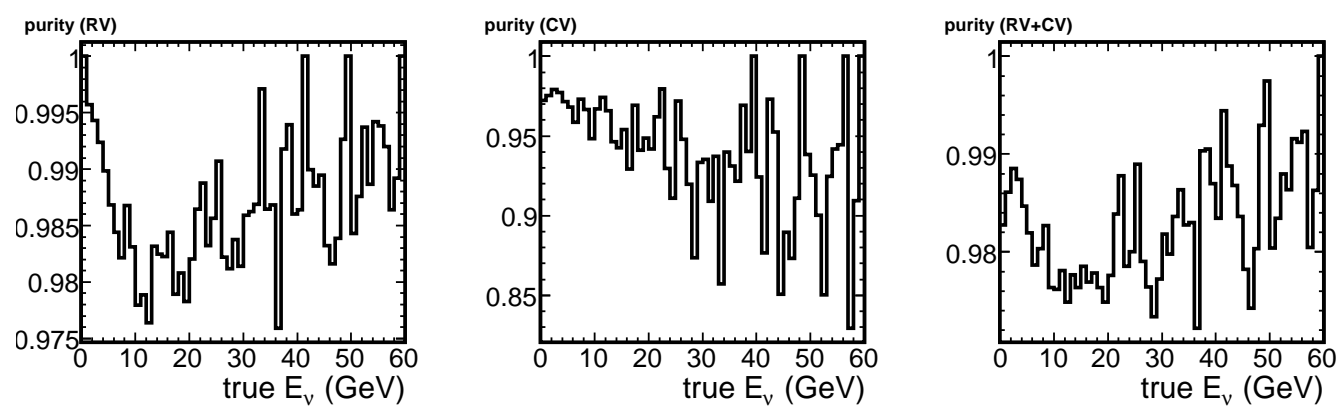

Figure 4.11: Charged current selection purity as a function of true neutrino energy. Average values: 98.7\% (Rock Vertex), 93.9\% (Contained Vertex), 98.3\% (Combined). Note the different vertical scales.

expected number of background events is known is $15 \%$. The expected number of cosmic ray muons in the final analysis data sample is $0.095 \pm 0.014$.

\subsubsection{Neutral current interactions}

This category of backgrounds arises from either a neutral current interaction, in which there is no muon created, or a charged current interaction, in which the muon path does not intersect the detector. In both cases, the charged particles leaving the interaction vertex can be reconstructed as a track in the detector. In almost all cases studied in the Monte Carlo, such a track is reconstructed from the passage of either a pion $\left(\pi^{ \pm}\right)$or a proton. A small fraction of these events do pass the antifiducial selection criteria and contaminate the muon sample.

As the MINOS detector has a different response to pions and protons than to muons, one could expect to develop a more sophisticated selection procedure to eliminate reconstructed tracks that did not come from the passage of a muon. This procedure has not been automated, but in fact has been carried out at the hand-scanning level by Marshak and Mann[61]. Such a procedure looks for higher energy deposition towards the end of the reconstructed track that is characteristic of charged pions and protons.

At the automated selection level, one possible criteria would be to cut events that fail to have a minimal track range requirement. This is a reasonable criteria, as the $d E / d X$ for pions and protons is generally higher than for muons. This is to say that a sample 
of muons with given energy $E$ will have a greater average range in the detector than a sample of pions with the same energy $E$. The impact of such a cut on this analysis was investigated. Ultimately, the performance of the analysis was degraded due to the fact that the lowest-momentum muons at the detector are the most sensitive to the oscillation parameters. Given the low level of NC contamination present in the antifiducial region, this track range cut was abandoned in favor of a more sophisticated PID cut, which labels events as "NC-like" or "CC-like". See Section 4.3 for a discussion of this selection procedure.

\subsubsection{Beam-induced $\tau$ interactions leading to muon detection}

Under the $\nu_{\mu} \rightarrow \nu_{\tau}$ oscillation hypothesis, some number of $\nu_{\tau}$ charged current interactions will take place for neutrinos in the beam above the $\tau$ production threshold. By using a model of $\nu_{\mu}$ and $\nu_{\tau}$ cross sections for the three charged current interactions (quasi-elastic, deep inelastic scattering, and resonance) a ratio $\eta\left(E_{\nu}\right)$ can be formed:

$$
\eta\left(E_{\nu}\right)=\frac{\left(\sigma_{Q E}\left(E_{\nu}\right)+\sigma_{D I S}\left(E_{\nu}\right)+\sigma_{R E S}\left(E_{\nu}\right)\right)_{\nu_{\tau}}}{\left(\sigma_{Q E}\left(E_{\nu}\right)+\sigma_{D I S}\left(E_{\nu}\right)+\sigma_{R E S}\left(E_{\nu}\right)\right)_{\nu_{\mu}}} .
$$

By convolving this cross section ratio with the neutrino spectrum giving rise to the nonfiducial events at the Far Detector, one obtains the number of $\tau$ leptons produced as a function of energy due to neutrino oscillation. Integrating over all neutrino energies, we have:

$$
N_{\tau}=\int_{0}^{\infty} d E_{\nu} \eta\left(E_{\nu}\right) N_{\mu}\left(E_{\nu}\right)
$$

The magnitude of this effect was estimated using the Monte Carlo in the following way. First, all selected muon events are assigned a weight equal to their oscillation probability $P\left(\nu_{\mu} \rightarrow \nu_{\tau}\right)$ at the Far Detector. Next, each event is weighted by the ratio $\eta\left(E_{\nu}\right)$ to represent the probability that a $\nu_{\tau}$ will give rise to a $\mathrm{CC}$ interaction. Then, each event is weighted by the total branching ratio for tau decays that give rise to a muon. The $\tau^{-} \rightarrow \mu^{-} \bar{\nu}_{\mu} \nu_{\tau}$ mode has $B R=0.1736$ and the $\tau^{-} \rightarrow \mu^{-} \bar{\nu}_{\mu} \nu_{\tau} \gamma$ mode has $B R=0.0036$ for a total $B R_{\mu}=0.1772$. This weighting procedure is finished if the original muon came from a contained vertex interaction, as any $\nu_{\tau}$ interaction in the detector will have the $\tau$ decay products detected. However, if the original muon came from a rock vertex interaction, one more weight is used to represent the fact that not all muons produced in such $\tau$ decays will reach the detector. In the three-body decay mode (ignoring the effect of the photon in $2 \%$ of these decays) we approximate the muon to 
carry one third of the initial $\tau$ energy on average. So the final weight applied is $1 / 3$ for all events in which the muon originates in the rock. This is equivalent to assuming that, relative to all analogous muon $\mathrm{CC}$ events, $2 / 3$ of muons produced from a tau $\mathrm{CC}$ event in the rock will range out before reaching the detector.

In both the rock vertex and contained vertex Monte Carlo sample, the expected tau contribution is quite small. Using the above method and scaling the MC exposure to $1.27 \times 10^{20} \mathrm{POT}, 0.2 \nu_{\tau}$ events from the rock vertex sample and 0.3 events from the contained vertex sample are expected 


\begin{tabular}{c|c|c|c|c}
\hline \hline Requirement & Open Data & Closed Data & MC Exp. & Cosmic \\
\hline no cuts & 19462 & 51328 & 1298.7 & 444.8 \\
\hline no LI activity & 3828 & 9058 & 1298.6 & 442.7 \\
good FD and beam quality & 3799 & 8981 & 1298.6 & 442.7 \\
ntrack $>0$ & 770 & 1442 & 1093.1 & 373.5 \\
good momentum measurement & 478 & 947 & 1000.4 & 107.9 \\
reco dircosneu $>$ 0.5 & 420 & 834 & 990.0 & 13.7 \\
track vtx z $<28.0 \mathrm{~m}$ & 405 & 810 & 963.7 & 13.1 \\
track vtx outside fiducial volume & 191 & 410 & 404.3 & 12.8 \\
pid0 $>-0.5$ & 166 & 372 & 360.6 & 12.4 \\
qp $\leq 0$ & 146 & 322 & 313.4 & 6.1 \\
$P_{\text {trans }}<2.5$ GeV/c & 140 & 302 & 309.1 & 1.2 \\
$P<10$ GeV/c & 109 & 238 & 252.9 & 1.2 \\
arrival time $(10 \mu$ s window $)$ & 105 & 232 & 252.9 & 0.095 \\
\hline selected events & 105 & 232 & 252.9 & 0.095 \\
below 3 GeV/c & 53 & 117 & 150.2 & 0.0 \\
\hline \hline selected as rock event & $78(74 \%)$ & $181(78 \%)$ & $199.1(79 \%)$ & 0.080 \\
selected as contained event & $27(26 \%)$ & $51(22 \%)$ & $53.8(21 \%)$ & 0.015 \\
\hline momentum from range & $92(88 \%)$ & $199(86 \%)$ & $210.0(83 \%)$ & $0.038(40 \%)$ \\
momentum from curvature & $13(12 \%)$ & $33(14 \%)$ & $42.9(17 \%)$ & $0.057(60 \%)$ \\
\hline outside fid. vol. in front & $47(45 \%)$ & $122(53 \%)$ & $111.2(44 \%)$ & $0.018(19 \%)$ \\
outside fid. vol. in sides & $58(55 \%)$ & $110(47 \%)$ & $141.7(56 \%)$ & $0.077(81 \%)$ \\
\hline NC expectation & - & & $4.1(1.6 \%)$ & - \\
$\tau$ expectation & - & - & $0.5(0.2 \%)$ & - \\
\hline \hline
\end{tabular}

Table 4.1: Summary of event selection criteria and the events remaining after each cut. "MC Exp." displays the expectation for events in the antifiducial volume when combining the RV and CV MC with the proper 1.27e20 POT weighting and no oscillation. The closed data set has a rate reduced from the expectation consistent with oscillation. "Cosmic" displays the observed event rate from all backgrounds during a live time of 5.2 days in February 2006 (see Section 4.5). The expected number of cosmic ray muon events in the selected sample is $0.095 \pm 0.014$. 


\begin{tabular}{|c|c|c|}
\hline \hline Requirement & RV MC & CV MC \\
\hline no cuts & 396.8 & 902.0 \\
\hline no LI activity & 396.8 & 902.0 \\
good FD and beam quality & 396.8 & 901.8 \\
ntrack $>$ 0 & 306.8 & 786.3 \\
good momentum measurement & 279.7 & 720.7 \\
reco dircosneu > 0.5 & 278.6 & 711.4 \\
track vtx z < 28.0 m & 275.2 & 688.5 \\
track vtx outside fiducial volume & 274.0 & 130.3 \\
pid0 > -0.5 & 265.4 & 95.2 \\
qp $\leq$ 0 & 229.2 & 84.2 \\
$P$ trans $<2.5$ GeV/c & 226.7 & 82.5 \\
$P<10$ GeV/c & 181.9 & 70.9 \\
arrival time $(10 \mu$ s window $)$ & 181.9 & 70.9 \\
\hline selected events & 181.9 & 70.9 \\
below 3 GeV /c & 108.2 & 41.9 \\
\hline \hline selected as rock event & 180.3 & 18.8 \\
selected as contained event & 1.6 & 52.1 \\
\hline momentum from range & $155.9(86 \%)$ & $54.1(76 \%)$ \\
momentum from curvature & $26.0(14 \%)$ & $16.8(24 \%)$ \\
\hline outside fid. vol. in front & $102.8(57 \%)$ & $8.4(12 \%)$ \\
outside fid. vol. in sides & $79.1(43 \%)$ & $62.5(88 \%)$ \\
\hline NC expectation & $1.5(0.8 \%)$ & $2.6(3.7 \%)$ \\
$\tau$ expectation & $0.2(0.1 \%)$ & $0.3(0.3 \%)$ \\
\hline \hline
\end{tabular}

Table 4.2: Summary of event selection criteria and the Monte Carlo events remaining after each cut - total events are scaled to the $1.27 \times 10^{20}$ POT data-equivalent exposure. "RV MC" displays the expectation for events modeled with an interaction vertex in the rock. "CV MC" labels those events modeled with an interaction vertex in the detector. 


\title{
Chapter 5
}

\section{Predicting the Far Detector event spectrum}

\author{
"I can't believe that!" said Alice. \\ "Can't you?" the queen said in a pitying tone. "Try again, draw a long \\ breath, and shut your eyes." \\ Alice laughed. "There's no use trying," she said. "One can't believe impos- \\ sible things." \\ "I dare say you haven't had much practice," said the queen. "When I was \\ your age, I always did it for half an hour a day. Why, sometimes I've believed \\ as many as six impossible things before breakfast." \\ -Lewis Carroll, Through the Looking Glass
}

The Monte Carlo model for rock vertex interactions in the MINOS Far Detector uses an input neutrino flux given by the GNuMI simulation (see Section 3.1). Studies comparing the modeled neutrino event rates in the Near Detector with that observed in ND data have resulted in a parameterized reweighting scheme called SKZP[55] based on the parent hadron kinematics. This results in a modeled FD flux that more accurately describes the neutrino energy spectrum observed at the ND.

For this analysis, a direct extrapolation of the ND data spectrum is used, instead of a Monte Carlo reweighting scheme. This provides a direct correction to the neutrino flux that was used in the GMINOS Far Detector and rock model, as opposed to a correction 
based on a parameterized fit to ND data. Several steps are required to obtain this correction:

- Measurement of the neutrino event spectrum at the Near Detector

- Conversion of the measured event rate to the true event rate based on the Monte Carlo model of the Near Detector response

- Extrapolation of the true ND event rate to the true FD event rate

- Correction to Monte Carlo event spectrum for contained vertex events

- Reweighting of MC rock vertex event spectrum using this same correction

After these steps are completed, the Monte Carlo expectation (with no oscillation modeled) for the Far Detector muon spectrum in the antifiducial region is obtained. This null hypothesis spectrum (no $\nu_{\mu}$ disappearance) can then be compared to the observed data spectrum to rule out the null hypothesis at some confidence level.

\subsection{Near detector measurement of neutrino spectrum}

As shown in Section 1.4.2, the contribution to the rock muon sample from high energy neutrinos is greater than in the contained vertex event sample, due to the extra power of energy in the event rate (see Equation 1.3). Because of this, caution must be exercised when predicting the high energy tail of the NuMI neutrino beam spectrum. Because of the entry of the parent hadron mass into the equation for the energy dependence of the neutrino (see Section B.2), the neutrinos that arise from charged kaons in the beam line have a higher average energy than those from the charged pions.

The Near and Far Detectors were designed with nearly identical composition and structure in order to reduce the systematic uncertainties involved in comparing the two measured neutrino spectra. The main difference relevant to this analysis is the physical dimension of each detector. Due to the smaller size of the Near Detector, obtaining a curvature measurement from a high momentum muon becomes difficult, and essentially impossible beyond a certain momentum. This value, known as the Maximum Detectable Momentum (MDM), has been described in detail in an internal note by T. H. Fields[63] and a cosmic-ray conference submission by M. Goodman[64]. While the argument as set forth was applied to the measurement of cosmic ray muons at the FD, it can be 
extended to the measurement of high energy muons from the NuMI beam at the ND. Fields defines the MDM as the value at which a muon with reconstructed momentum equal to the MDM has an $86 \%$ probability of having a true momentum greater than half the MDM (assuming a Gaussian distribution of curvature measurements).

The direct measurement made in the MINOS detectors is that of the amount of curvature experienced by a charged particle in the magnetic field. Specifically, we measure the quantity $q / p$, or the curvature (inverse momentum) times the charge of the particle (determined by the direction of bending within the detector and our knowledge of the magnetic field orientation). The reconstruction software assigns an uncertainty on this curvature measurement $\delta_{q / p}$ for each event. We can interpret this value as the inverse of the MDM that the software is "comfortable" dealing with, given the pattern of strip hits under consideration. When the confidence in the reconstructed track quality is low, this uncertainty will be large, and the MDM will be low relative to a clean event. By taking an ensemble of beam track events at the ND, we can use the average $1 / \delta_{q / p}$ value as a determination of the MDM for the detector. Figure 5.1 displays this distribution for Near Detector data for a sample of $\sim 116,000$ selected events. The median value gives a Maximum Detectable Momentum of $40 \mathrm{GeV}$ when integrated over the entire neutrino energy spectrum.

However, this distribution is heavily weighted by the low-energy peak in the neutrino energy spectrum. Two distinct contributions are shown in Figure 5.2. The first is that of muons with a track length less than or equal to 180 planes (the first peak near $20 \mathrm{GeV} / \mathrm{c}$ ) that do not travel far into the spectrometer region of the ND. The "lever arm" for measuring the curvature of such muons is limited, and they display greater uncertainty in this measurement, with a median value $1 / \delta_{q / p}=29 \mathrm{GeV} / \mathrm{c}$. However, muons in this group do not deposit more than about $8 \mathrm{GeV}$ before ranging out in the detector. The vast majority lie in the focusing peak around $2-6 \mathrm{GeV}$, so this MDM is sufficient to properly determine the curvature of most muons to $\sim 3 \sigma$ confidence.

The second contribution is from muons with a track length greater than 180 planes, which reach well into the spectrometer. Such tracks can only deposit about $12 \mathrm{GeV}$ before exiting the detector. This group has a median value $1 / \delta_{q / p}=167 \mathrm{GeV} / \mathrm{c}$. The sample of long muon tracks demonstrates that the Near Detector is capable of resolving the high-energy tail of the neutrino energy spectrum quite well for muons that are focused into the spectrometer. 


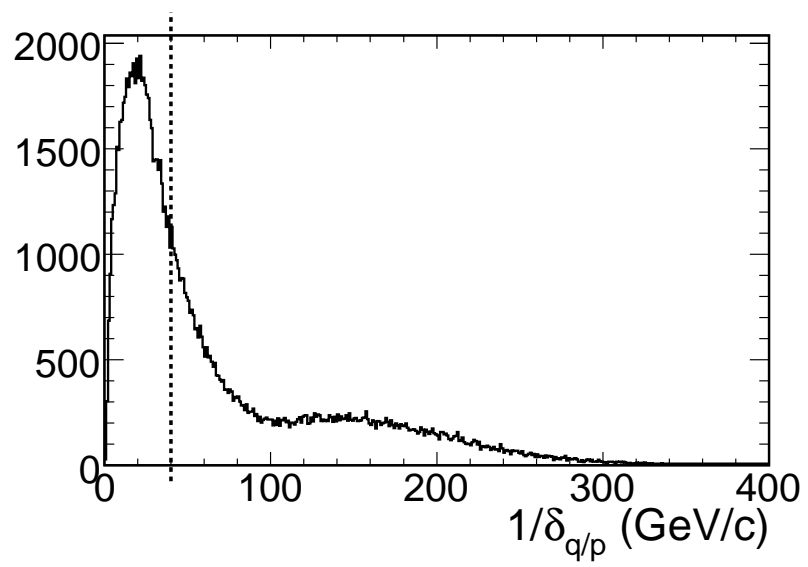

Figure 5.1: Maximum Detectable Momentum in the MINOS Near Detector. The dotted line indicates the median of the distribution.

\subsection{Extrapolation method}

The Near Detector data together with the Monte Carlo can be used to predict the Far Detector muon spectrum. The final SKZP PiMinus parameterization[55] is used to reweight the ND MC to more closely resemble the reconstructed data spectrum. The reweighting is accomplished by fitting the generated MC to the observed data in bins of $x_{F}$ (the fraction of the initial proton energy carried by the parent hadron) and $P_{t}$ (the transverse momentum of the parent hadron). The better this reweighted MC models the actual ND energy spectrum, the better the extrapolation will predict the FD spectrum.

There are four steps which utilize the ND MC to make a conversion from the reconstructed neutrino spectrum to a true $\mathrm{CC} \nu_{\mu}$-only spectrum:

1. Correction for CC purity, $P\left(E_{\text {reco }}\right)$.

2. Conversion from reconstructed to true energy, $M\left(E_{\text {true }} \times E_{\text {reco }}\right)$.

3. Correction for selection efficiency, $\epsilon\left(E_{\text {true }}\right)$.

4. Correction for slicing effects, $S\left(E_{\text {true }}\right)$.

After these steps are complete, an estimate of the true CC $\nu_{\mu}$ spectrum at the ND is obtained. This spectrum is then extrapolated to the Far Detector by means of the F/N 


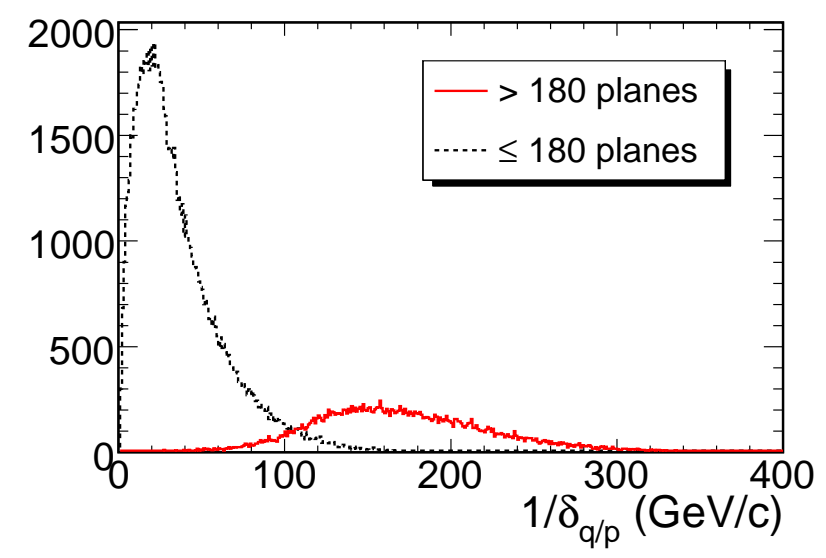

Figure 5.2: Maximum Detectable Momentum in the MINOS Near Detector, divided into two track length categories. Short tracks are shown in the dashed black line, and long tracks are shown in the solid red line.

flux ratio (derived from the v18 flux files). The resulting FD spectrum in true $E_{\nu}$ is used to provide a correction to the flux which was fed into the FD Monte Carlo. That correction yields a weight for each muon event which is based upon the parent neutrino energy. In this manner, the reconstructed FD muon spectrum in the antifiducial volume is reweighted to be consistent with the ND measurement of the neutrino spectrum.

Neutrino event rate distributions are defined as $\Phi=c \phi \sigma$, where $c$ is a proportionality constant, $\phi$ is the neutrino flux distribution, and $\sigma$ is the total cross section. In this extrapolation method, it is assumed that $c \sigma$ is identical in the Near and Far Detectors, such that $\Phi^{F D} / \Phi^{N D}=\phi^{F D} / \phi^{N D}$. The following labels are defined for the event rate distributions used in the extrapolation:

- $\Phi_{0} \equiv \Phi_{\text {data }}^{f a r}\left(E_{\text {true }}\right)$, an estimate of the true data neutrino event spectrum at the FD.

- $\Phi_{1} \equiv \Phi_{M C}^{f a r}\left(E_{\text {true }}\right)$, the input true neutrino event spectrum at the FD used in GMINOS.

- $\Phi_{2} \equiv \Phi_{\text {data }}^{\text {near }}\left(E_{\text {reco }}\right)$, the data spectrum as reconstructed at the ND.

- $\Phi_{2}^{\prime} \equiv \Phi_{\text {data }}^{\text {pure near }}\left(E_{\text {reco }}\right)=P\left(E_{\text {reco }}\right) \Phi_{2}$, the pure $\nu_{\mu}$ CC spectrum. 
- $\Phi_{2}^{\prime \prime} \equiv \Phi_{\text {data }}^{\text {pure near }}\left(E_{\text {true }}\right)=M\left(E_{\text {true }} \times E_{\text {reco }}\right) \Phi_{2}^{\prime}$, converted to true neutrino energies.

- $\Phi_{2}^{\prime \prime \prime} \equiv \Phi_{\text {data }}^{\text {actual pure near }}\left(E_{\text {true }}\right)=\Phi_{2}^{\prime \prime} / \epsilon\left(E_{\text {true }}\right)$, the efficiency-corrected spectrum.

- $\Phi_{3} \equiv \Phi_{\text {data }}^{\text {near }}\left(E_{\text {true }}\right)=S\left(E_{\text {true }}\right) \Phi_{2}^{\prime \prime \prime}$, an estimate of the true data spectrum at the $\mathrm{ND}$, corrected for time-slicing effects.

- $\Phi_{4} \equiv \Phi_{M C}^{n e a r}\left(E_{r e c o}\right)$, the reconstructed ND MC spectrum.

- $\Phi_{5} \equiv \Phi_{M C}^{n e a r}\left(E_{t r u e}\right)$, the input true ND spectrum used in GMINOS MC.

All plots in this section should be understood to extend to $120 \mathrm{GeV}$ when used in this analysis. They are displayed up to $60 \mathrm{GeV}$ for clarity of the relevant region producing the majority of events at our current exposure. All plots describing the steps derived from Near Detector Monte Carlo display the relevant MC event spectra (Figures 5.3 - 5.6. The Near Detector data spectrum is introduced after the MC processing steps are complete, in Figure 5.8. Statistical fluctuations in the plots at high energy/momentum are due to the low number of MC events generated in these regions. Where appropriate, these low-statistics bins were grouped into larger bins for the purpose of the extrapolation.

\subsubsection{Correction for CC purity}

The selection for CC-like events at the ND (pid0 $>-0.1)$ admits some neutral current background into the analysis data sample. In a similar way, the charge sign cut $(q p \leq 0)$ admits some positive muons into the sample. To correct for these effects, the Monte Carlo $\nu_{\mu} \mathrm{CC}$ purity is calculated in bins of reconstructed energy; this is labeled as $P\left(E_{\text {reco }}\right)$. The reconstructed data spectrum is multiplied bin-by-bin by this purity correction. Figure 5.3 demonstrates this step as applied to the reconstructed ND MC. The plot on the left displays $P\left(E_{\text {reco }}\right)$, while the plot on the right displays $\Phi_{2}$ ("Reco CC-like") and $\Phi_{2}^{\prime}=$ $P\left(E_{\text {reco }}\right) \Phi_{2}$ ("Reco pure CC"). This correction effectively removes the residual NC events and positive muons in each bin of reconstructed energy (mainly present at low energy). The end result of this step is the pure $\mathrm{CC} \nu_{\mu}$ spectrum as measured at the ND.

\subsubsection{Conversion from reconstructed to true energy}

The resulting pure $\mathrm{CC} \nu_{\mu}$ spectrum is then taken as a column vector with $j$ elements and left-multiplied by the matrix displayed on the left in Figure 5.4. This detector matrix, $M\left(E_{\text {true }} \times E_{\text {reco }}\right)$, is formed using all true $\mathrm{CC} \nu_{\mu}$ events in the fiducial volume of the ND 

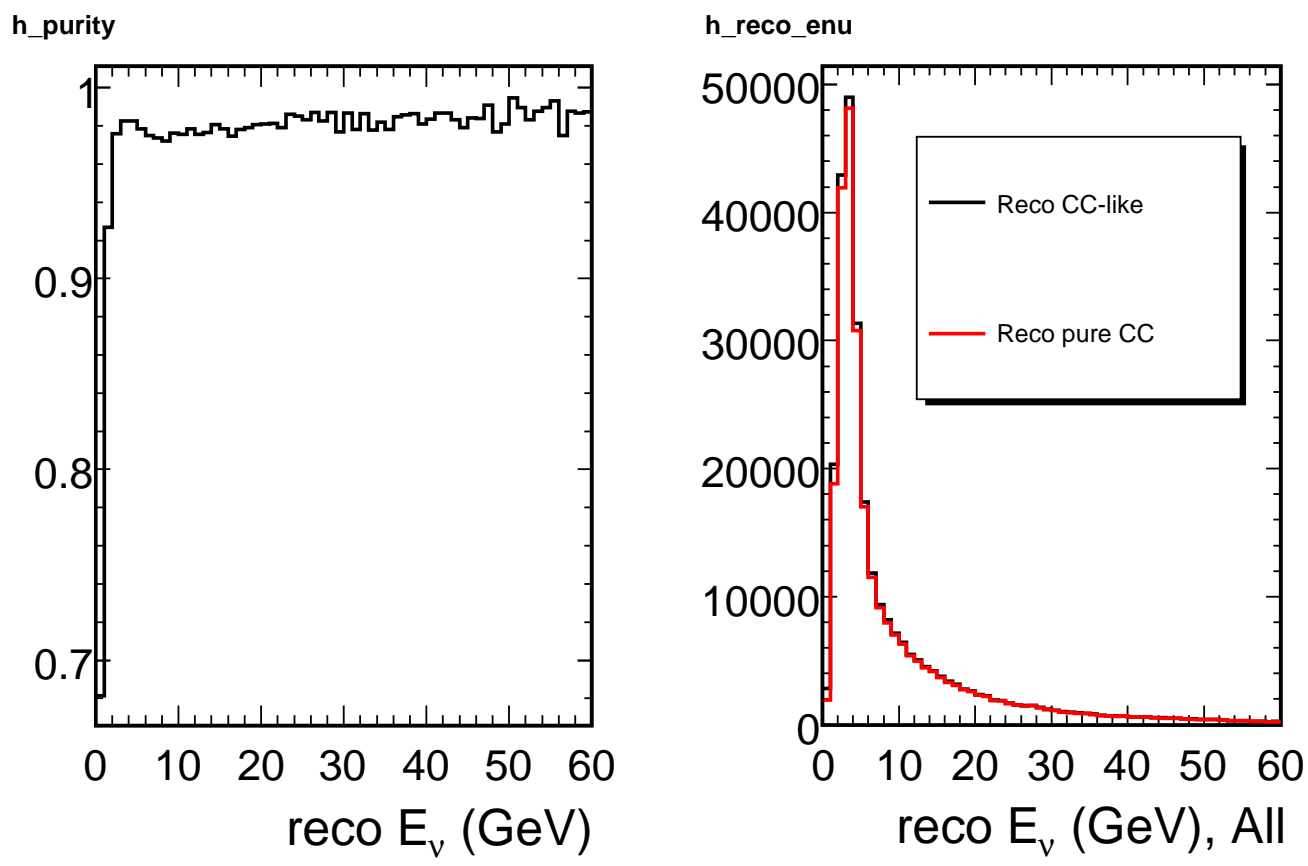

Figure 5.3: Left: $\mathrm{CC} \nu_{\mu}$ purity as a function of reconstructed energy as modeled in Monte Carlo. Right: Selected CC-like events before (solid black line, "Reco CC-like") and after (solid red line, "Reco pure CC-like")the purity correction.

as modeled in Monte Carlo. A histogram of true vs. reconstructed energy is filled first. Then each column of the matrix (one bin in reconstructed energy, $E_{\text {reco }}^{j}$ ) is normalized to one, such that each bin $E_{\text {true }}^{i}$ within this column gives the probability that a neutrino of energy $E_{\text {true }}^{i}$ will be reconstructed with energy $E_{\text {reco }}^{j}$. The resulting matrix multiplication gives a row vector with $i$ elements, representing the true neutrino spectrum reconstructed by the ND. The plot on the right in Figure 5.4 displays $\Phi_{2}^{\prime}$ ("Reco pure CC" from the last step) and $\Phi_{2}^{\prime \prime}=M\left(E_{\text {true }} \times E_{\text {reco }}\right) \Phi_{2}^{\prime}$ ("True pure CC").

\subsubsection{Correction for selection efficiency}

This analysis assumes that the response of the Near and Far Detectors to the neutrino beam is identical. In this way, the energy-dependent efficiency to detect and reconstruct 

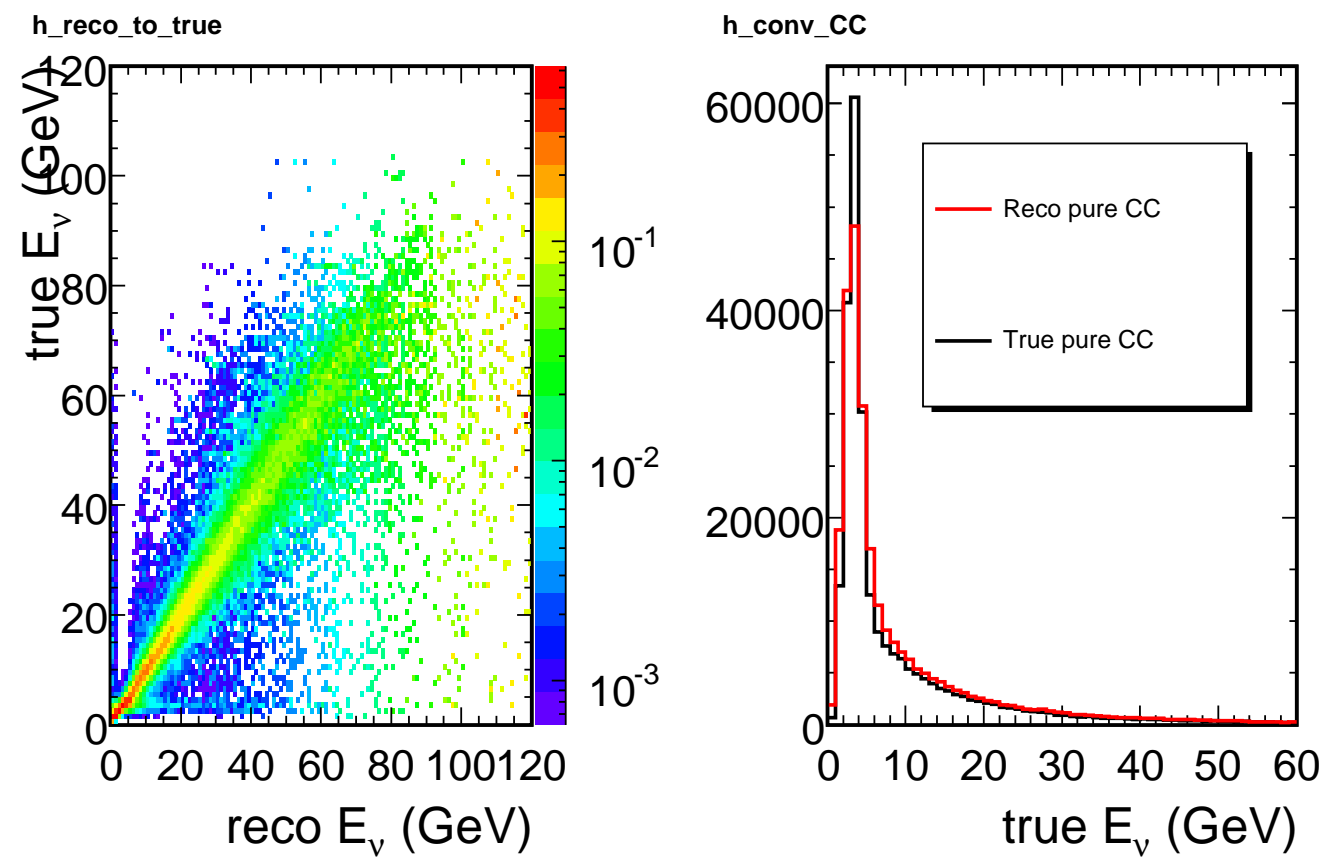

Figure 5.4: Left: Reconstructed neutrino energy vs. true energy for true CC $\nu_{\mu}$ events. Right: Pure CC spectrum as reconstructed energy (solid red line, "Reco pure CC"), and after (solid black line, "True pure CC") correction to true energy.

a neutrino interaction cancels out between the two detectors. The efficiency $\epsilon\left(E_{\text {true }}\right)$ is defined here relative to the events that are reconstructed with a vertex inside of the ND fiducial volume, with a reconstructed neutrino energy less than $120 \mathrm{GeV}$, and at least one reconstructed track. The cuts (and their respective efficiencies integrated over the spectrum) that are reflected in this selection efficiency are the charge sign cut $(81.3 \%)$, the PID cut (79.1\%), and the track quality cut (91.7\%). The spectrum obtained from the previous step is divided bin-by-bin by $\epsilon\left(E_{\text {true }}\right)$ to arrive at the true $\nu_{\mu}$ CC event spectrum. The left plot in Figure 5.5 displays this efficiency correction. The plot on the right displays $\Phi_{2}^{\prime \prime}$ ("True pure CC" from the last step) and $\Phi_{2}^{\prime \prime \prime}=\epsilon\left(E_{\text {true }}\right) \Phi_{2}^{\prime \prime}$ ("True pure CC corrected"). 

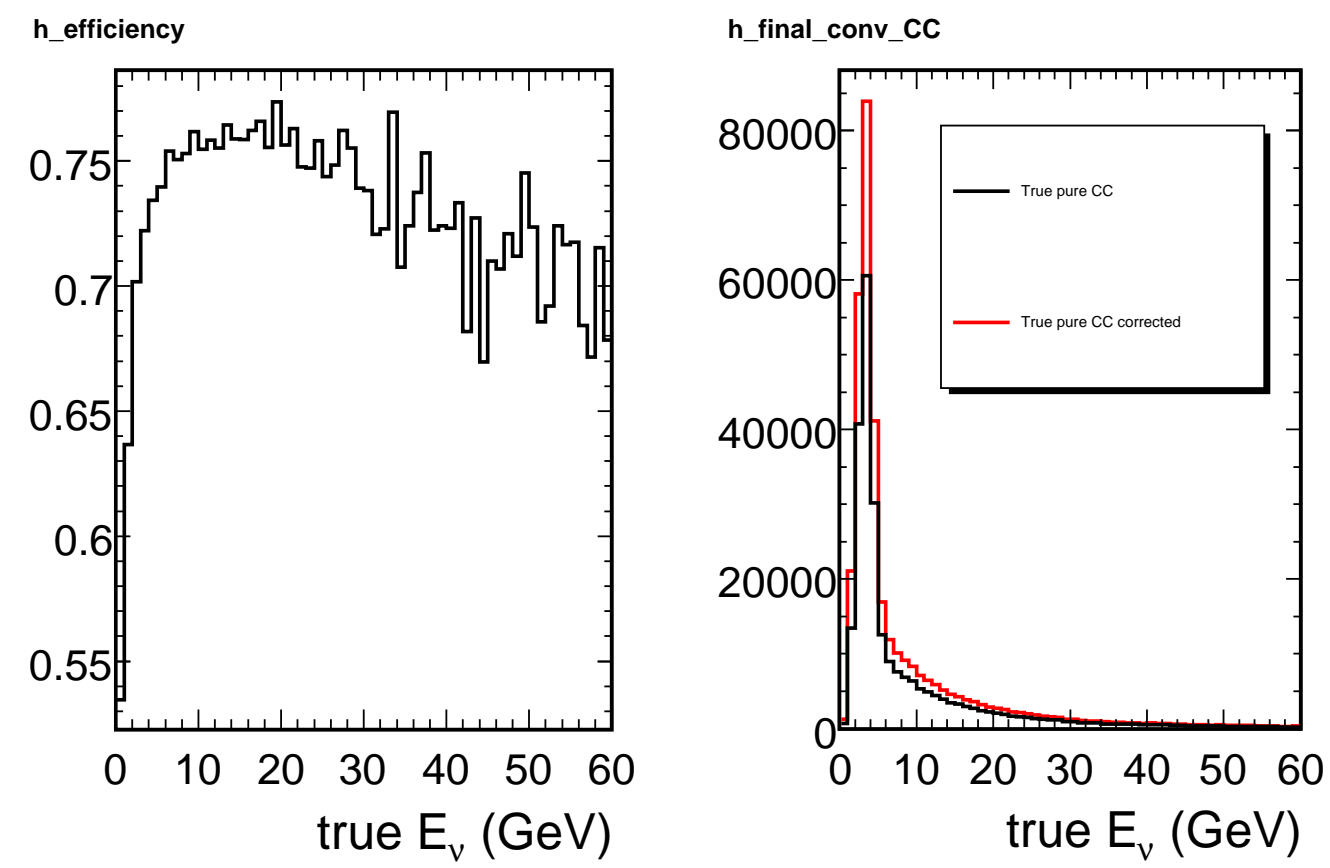

Figure 5.5: Left: selection efficiency as a function of true energy as modeled in Monte Carlo. Right: Measured spectrum at ND in true neutrino energy (solid black line, "True pure CC") and the actual spectrum (solid red line, "True pure CC corrected") after efficiency correction.

\subsubsection{Correction for slicing effects}

When one plots the reconstructed neutrino spectrum at the ND for both single event Monte Carlo and for full overlay files (which contain multiple events per snarl, to accurately represent the detector activity during the beam spill), it becomes apparent that there is a higher event rate in the overlay files, given the same POT exposure as in the singles files. This effect increases roughly linearly with energy, and appears to be due to improper slicing of long tracks into multiple events. N. Saoulidou has studied this effect and provided me with an energy-dependent correction $S\left(E_{\text {true }}\right)$ (see Figure 5.6), which is formed by the ratio of the single event spectrum over the overlay event spectrum. This is applied to the true $\nu_{\mu}$ CC spectrum $\Phi_{2}^{\prime \prime \prime}$ that results from the efficiency step to obtain 
$\Phi_{3}=S\left(E_{\text {true }}\right) \Phi_{2}^{\prime \prime \prime}$, the final estimate of the actual $\nu_{\mu}$ CC spectrum in true neutrino energy at the Near Detector.

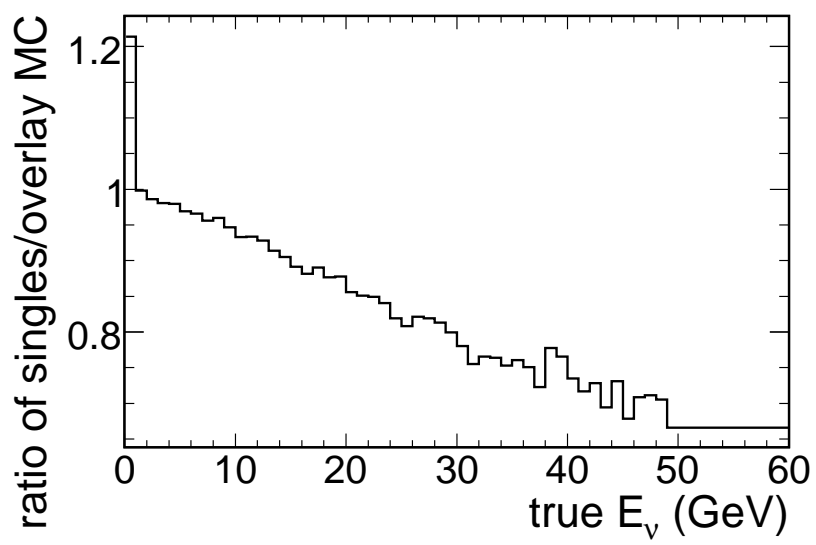

Figure 5.6: Weights applied to true neutrino energy spectrum to correct for double counting of events improperly sliced in time.

\subsubsection{Extrapolation using Far/Near flux ratio}

After all processing of the ND data is accomplished, the resulting estimate of the true $\nu_{\mu}$ CC event spectrum $\Phi_{3}$ at the Near Detector (shown on the left in Figure 5.8) is multiplied bin-by-bin with the Far/Near flux ratio $\phi_{1} / \phi_{5}$, shown in Figure 5.7. This ratio is constructed using the following procedure:

- A histogram of the $\nu_{\mu}$ flux at the ND is filled over nenergynear with a weight for each event of nimpwt $\times$ nwtnear, where nenergynear is the neutrino energy at the Near Detector, nimpwt is an importance weight which corrects for low statistics generated in certain regions of the neutrino spectrum, and nwtnear is a weight which represents the probability that a given neutrino will intersect the Near Detector.

- A histogram of the $\nu_{\mu}$ flux at the FD is filled over nenergyfar with a weight for each event of nimpwt $\times$ nwtfar, where the variables for the Far Detector are analogous to those at the Near Detector. 
- A histogram is then filled with the bin-by-bin ratio of the far flux over the near flux.

- Multiplying the true ND spectrum with this ratio histogram results in the predicted neutrino event spectrum at the FD after two required corrections. The resulting spectrum is first scaled by 2.642 , the ratio of exposures for the data used from the FD (1.27e20 POT) over the ND $(0.48 \mathrm{e} 20$ POT). The resulting spectrum is then scaled by 89.265 , the ratio of the FD fiducial mass $(3.94 \mathrm{kT})$ over the ND fiducial mass $(0.044 \mathrm{kT})$.

Justification for using the flux ratio for extrapolation, rather than the event ratio, is given by the assumption that the cross section for $\nu_{\mu} \mathrm{CC}$ interactions in the ND and FD are identical. This assumption is made possible by the nearly identical construction techniques used in the two detectors.

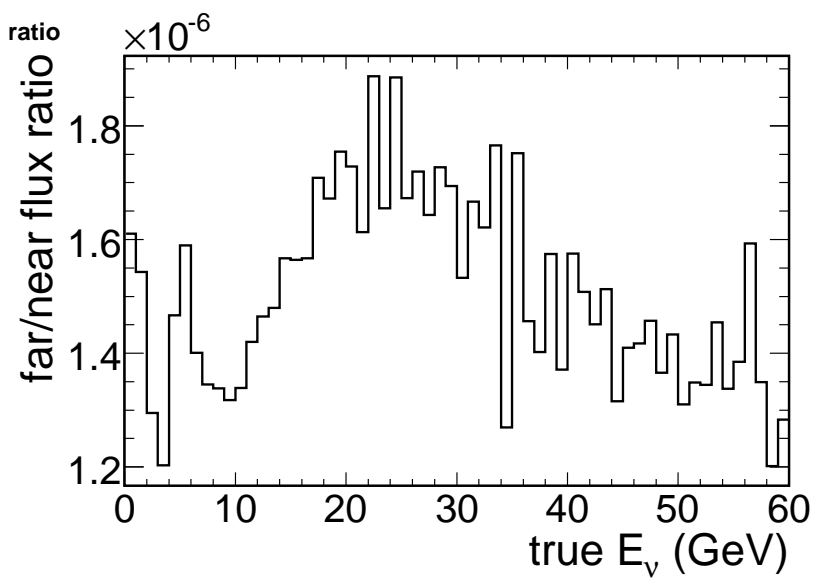

Figure 5.7: Far over near neutrino flux ratio used for extrapolation.

The predicted Far Detector event spectrum $\Phi_{0}=\Phi_{3} \times \phi_{1} / \phi_{5}$ resulting from this extrapolation is displayed on the right in Figure 5.8. Notice the slight broadening of the peak as compared to the Near Detector spectrum on the left. This is the main shape difference between the near and far spectra, resulting from known kinematic effects in the NuMI beam line. 

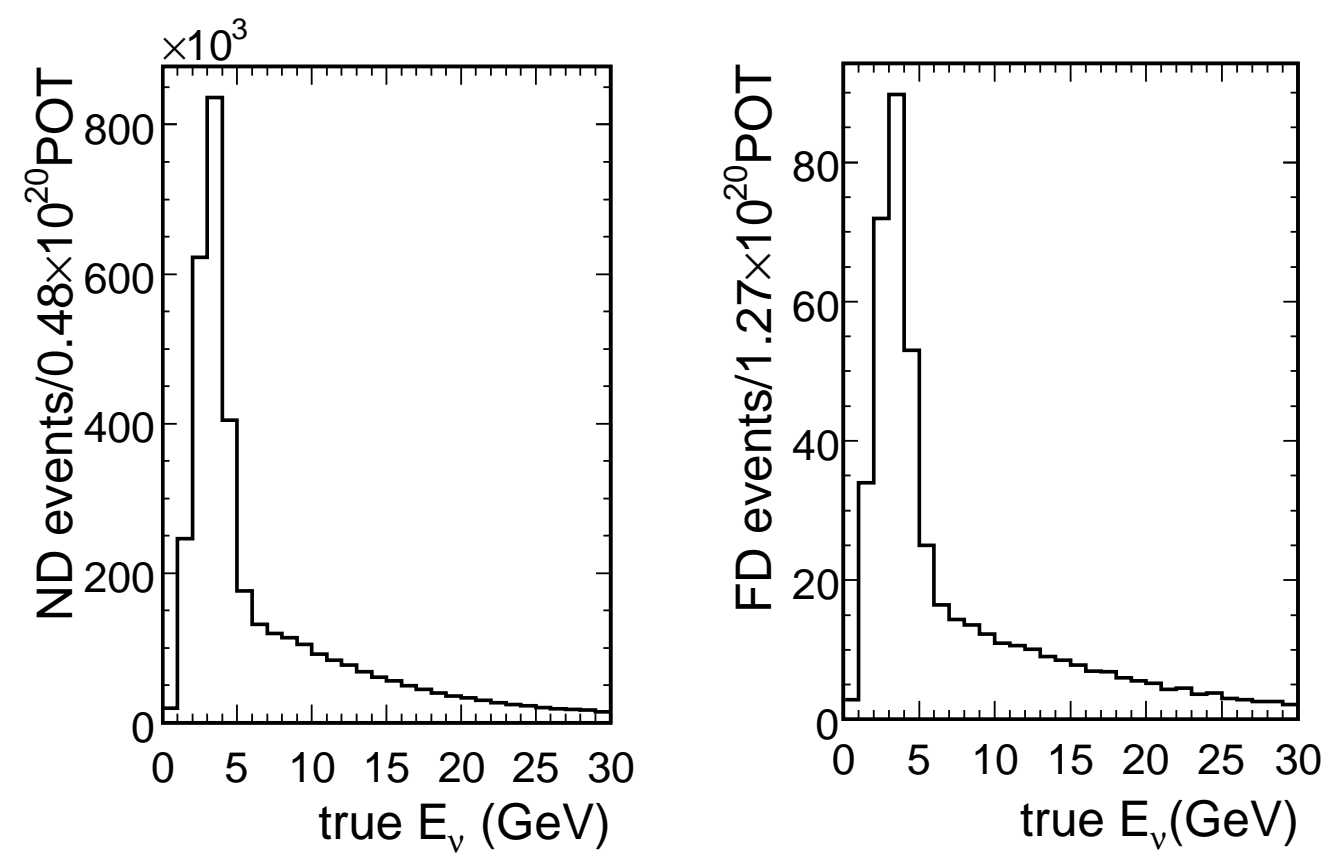

Figure 5.8: Near Detector data extrapolation. Left: True ND $\nu_{\mu}$ CC event spectrum after the described processing steps; Right: Predicted True FD $\nu_{\mu} \mathrm{CC}$ event spectrum after extrapolation step.

\subsubsection{Obtaining event weights from true $E_{\nu}$ prediction}

Given the predicted true $\nu_{\mu}$ CC event spectrum at the FD $\Phi_{0}$ (see Figure 5.8) that results from this extrapolation method, a comparison is made to the input true spectrum $\Phi_{1}$ used to generate the nominal FD Monte Carlo. In bins of true neutrino energy, the ratio of the predicted spectrum over the input spectrum is taken to form a weight $w\left(E_{\text {true }}\right)=\Phi_{0} / \Phi_{1}$. This weight provides the correction to the FD Monte Carlo $\nu_{\mu} \mathrm{CC}$ spectrum that is derived from the measured spectrum at the ND. Figure 5.9 displays this correction $w\left(E_{\text {true }}\right)$ in bins of true neutrino energy. 


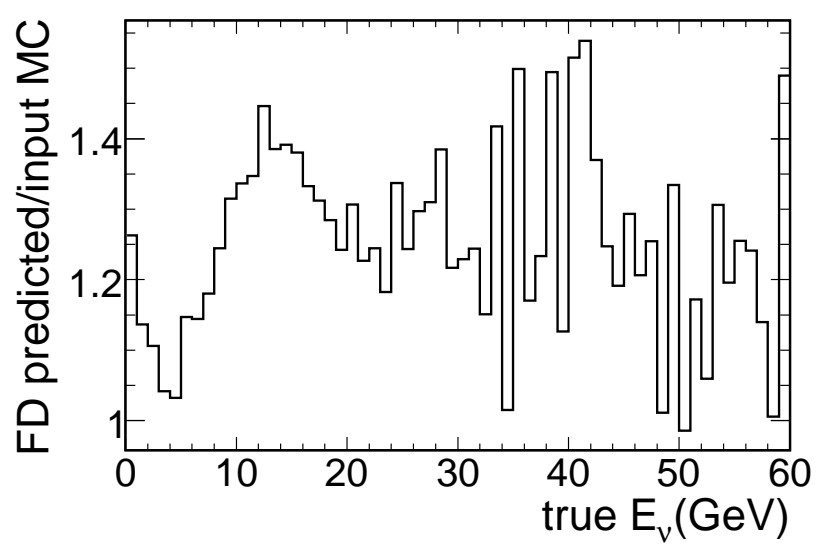

Figure 5.9: Ratio of predicted FD $\nu_{\mu} \mathrm{CC}$ event spectrum (using ND data extrapolation) to the input spectrum used in the nominal FD Monte Carlo.

\subsubsection{Final reconstructed $P_{\mu}$ prediction}

Now that the correction $w\left(E_{\text {true }}\right)$ for each bin of true $E_{\nu}$ is obtained, the following procedure is used:

- The Far Detector Monte Carlo events with a reconstructed track vertex outside the fiducial volume (both true contained vertex and true rock vertex events) are run through the selection cuts described in Section 4.2.

- Any track event which passes the selection criteria has a known true parent neutrino energy. The appropriate weight $w\left(E_{\text {true }}\right)$ for this bin of energy is selected from the histogram in Figure 5.9.

- The weighted event is placed in a 2-D histogram of reconstructed track momentum versus cosine of the angle between the reconstructed track vertex direction and the neutrino beam direction. For the eventual oscillation fit, there are ten bins in $P_{\text {reco }}$ from $0-10 \mathrm{GeV} / \mathrm{c}$ and ten bins in $\cos \left(\theta_{\text {reco }}\right)$ from $0.5-1.0$, resulting in 100 bins in two dimensions with equal size in the reconstructed variable space. 


\subsection{Resulting muon predictions}

When the above prescription is carried out on Near Detector data and Far Detector Monte Carlo, the following distributions are predicted in the absence of $\nu_{\mu}$ disappearance. Figure 5.10 displays the expected muon distribution up to $30 \mathrm{GeV} / \mathrm{c}$, Figure 5.11 displays the expected angular distribution, and Figure 5.12 displays the expected two-dimensional distribution when binned as described in the previous section. Notice the correlation between reconstructed momentum and angle: the high momentum muons tend to strike the detector head-on, at $\cos \theta_{\text {beam }} \sim 1$, while the less energetic muons are distributed to the lower angle bins. As was shown in Figure 4.1, the event rate drops off exponentially with decreasing $\cos \theta_{\text {beam }}$.

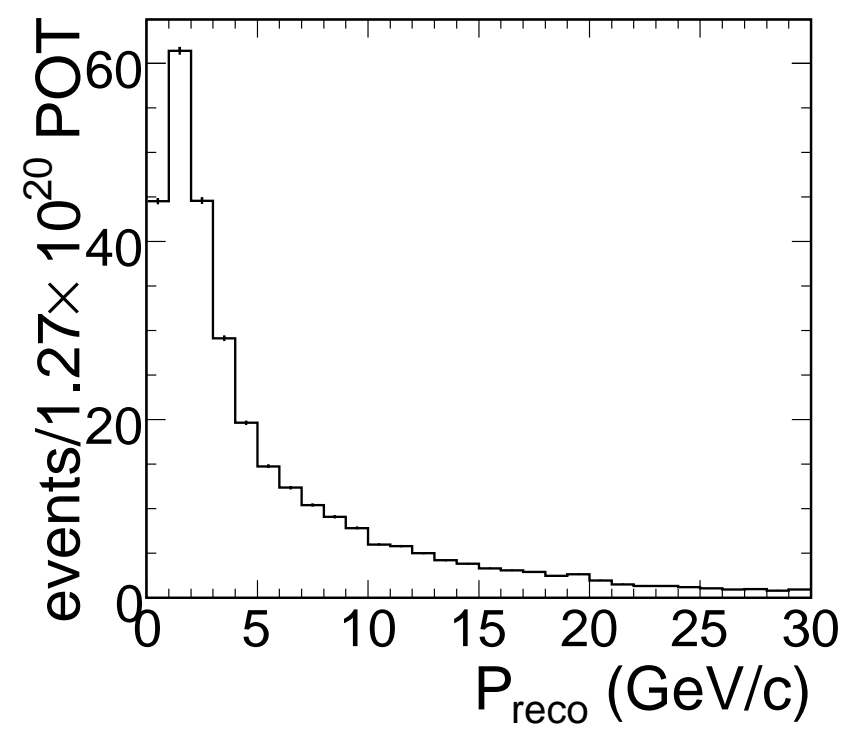

Figure 5.10: Expected reconstructed muon momentum distribution for $1.27 \times 10^{20}$ POT.

The resulting one-dimensional distributions of reconstructed track momentum and angle will be used to perform consistency checks between the data and Monte Carlo. Ultimately, the two-dimensional MC distribution will be compared to the data in the hypothesis test and oscillation fit. 


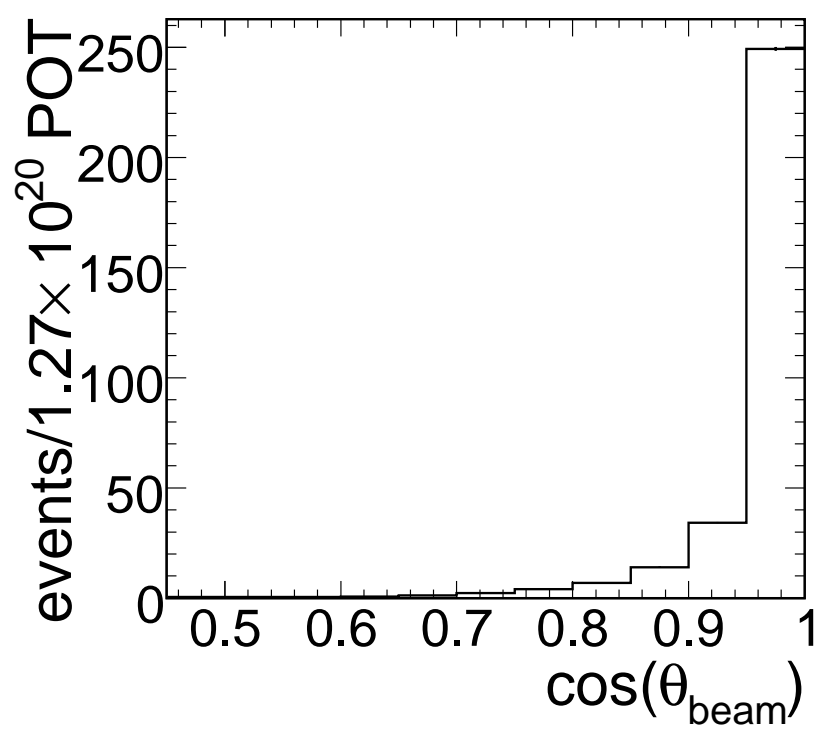

Figure 5.11: Expected reconstructed muon direction distribution for $1.27 \times 10^{20}$ POT. 


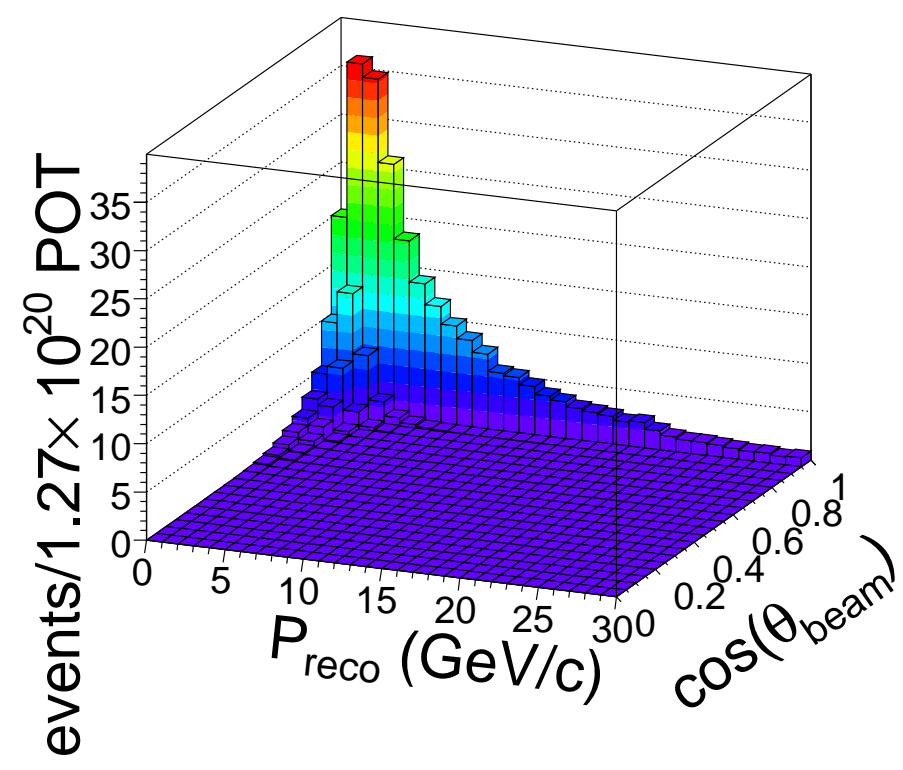

Figure 5.12: Expected reconstructed muon distribution for $1.27 \times 10^{20}$ POT in twodimensional histogram of momentum and direction. 


\section{Chapter 6}

\section{Mock data tests}

The first principle is that you must not fool yourself - and you are the easiest person to fool.

-Richard Feynman

In preparation for box-opening of the final data set, the analysis was exercised on several "mock data" Monte Carlo samples. First, sensitivities were estimated using three different fit procedures. The final analysis uses the third fit procedure, which utilizes information from the rate, momentum, and direction of reconstructed tracks. Sensitivities were obtained for various combinations of input oscillation parameters. Finally, the systematic uncertainties on the measurement of the oscillation parameters were evaluated and summed in quadrature to estimate the final systematic uncertainty.

Since the magnitude of systematic uncertainty depends on the true oscillation parameters, this evaluation was calculated once before the box opening for input oscillation parameters equal to the best fit published[1] by the MINOS collaboration in 2006. It was then re-calculated at the best fit parameters found in the antifiducial analysis (presented in Chapter 7). The systematic uncertainties presented here are those calculated for the antifiducial best fit result.

\subsection{Fit procedure}

The fit procedure for this analysis is based on Poisson statistics and assumes the event rate in each bin to be independent. A comparison between the expected number of events (derived from the FD Monte Carlo and ND data) and the observed number of events in 
the data forms the basis of the fit. Appendix C.1 describes in detail the log likelihood method that is used in this comparison. Three binning methods were studied:

1. One bin only is used to compare the total rate between expected and observed events.

2. The power of the 1-D momentum distribution is added to the rate measurement.

3. The additional information contained in the angular distribution is added to the momentum and rate measurement to form a 2-D distribution.

The relative significance levels (statistical only) at which each fit rules out the no disappearance hypothesis (at $1.27 \times 10^{20}$ POT) were estimated at $7 \%$ for the rate-only test, $3 \%$ for the rate plus momentum test, and $1 \%$ for the $2-\mathrm{D}$ rate, momentum, and angle test. These are representative numbers for the tested range of oscillation parameters indicated by previous experiments $\left(\left|\Delta m_{32}^{2}\right|=2.0-3.0 \times 10^{-3} \mathrm{eV}^{2} / \mathrm{c}^{4}, \sin ^{2} 2 \theta_{23}=0.9-\right.$ 1.0).

By plotting the unoscillated and oscillated spectra for the parent neutrino energy, reconstructed muon energy and direction, one can visualize the relative sensitivity of the antifiducial muon analysis and the fiducial neutrino analysis, as well as the added sensitivity using the momentum and direction distribution. Figure 6.1 displays these three plots, as well as the ratio of oscillated/unoscillated spectra for full Monte Carlo statistics. The left plots display this information for the parent neutrino spectra, which are not available in the observed data for the antifiducial analysis. The depth of the dip is related to the value of $\sin ^{2} 2 \theta_{23}$, as this term appears as the amplitude of $\sin ^{2}\left(1.27 \Delta m_{32}^{2} \frac{L}{E}\right)$ in the survival probability formula (Equation B.18); the location of the dip is related to the value of $\left|\Delta m_{32}^{2}\right|$, as this appears as the argument of $\sin ^{2}\left(1.27 \Delta m_{32}^{2} \frac{L}{E}\right)$.

The shape of the ratio plots in Figure 6.1 for muon energy and direction dictate the extra information gained by adding these distributions to the rate-only fit. The center plot indicates that the deficit information is present almost entirely for muons reconstructed below $5 \mathrm{GeV} / \mathrm{c}$. The right plot indicates that there is a nearly flat deficit over the range of track angles, but some shape information is present at the two ends of the range. A perfectly flat distribution would add no information to the rate-only fit.

A simple counting experiment achieves some sensitivity to the oscillation parameters. While the fit will return some combination of best-fit $\left|\Delta m_{32}^{2}\right|$ and $\sin ^{2} 2 \theta_{23}$, an upper limit on $\left|\Delta m_{32}^{2}\right|$ in the region of the atmospheric mixing parameters is not attainable. Such an 


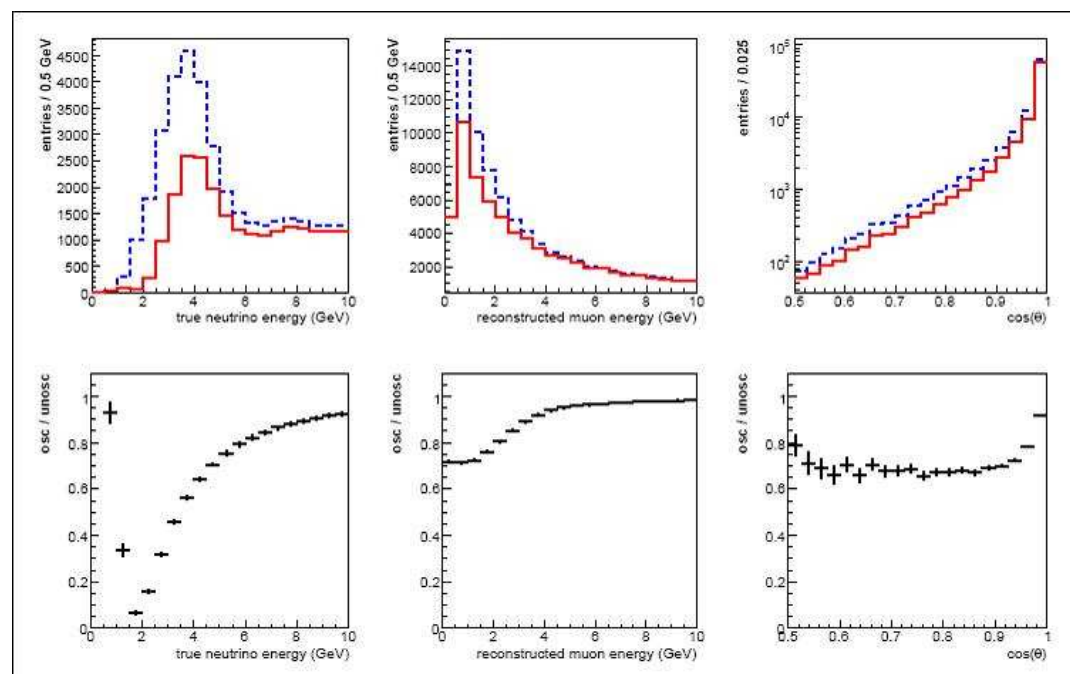

Figure 6.1: Top row: oscillated (solid red line) and unoscillated (dotted blue line) MC spectra for parent neutrino energy (left), reconstructed muon energy (center) and direction (right). Bottom row: ratio of oscillated/unoscillated spectra for the three corresponding plots.

analysis corresponds to a measurement of the average oscillation probability $\langle P\rangle$, which leads to an exclusion boundary in the parameter space at a vertical line $\sin ^{2} 2 \theta_{23}=2\langle P\rangle$ above a value of $\left|\Delta m_{32}^{2}\right| \sim 6 \times 10^{-3} \mathrm{eV}^{2} / \mathrm{c}^{4}$. The information present in the momentum and angle distributions of selected track events will result in increased sensitivity, i.e. a reduced region of oscillation parameters allowed and closed contours in the parameter plane.

\subsubsection{Rate, momentum, and direction}

The full sensitivity in this analysis is achieved by including the absolute rate in bins of reconstructed momentum and direction. A simultaneous fit is performed in order to take full advantage of the correlation that exists between the momentum and angle of the muon candidates. This procedure is accomplished in the following way:

- The relevant truth and reconstructed quantities for all selected Monte Carlo events (corresponding to an exposure of $\sim 300 \times 10^{20}$ POT) are stored in memory as the 2 -D histogram is filled, event-by-event, in $P_{\text {reco }}$ versus $\cos \left(\theta_{\text {beam }}\right)$. These include 
reconstructed track momentum, reconstructed track direction, true neutrino energy and flavor, and the type of interaction (CC or $\mathrm{NC}$ ).

- The oscillation parameter space is divided into a grid, with 800 points in $\Delta m_{32}^{2}$ from 0.0 to $8.0 \times 10^{-3} \mathrm{eV}^{2} / \mathrm{c}^{4}$, and 100 points in $\sin ^{2} 2 \theta_{23}$ from 0.0 to 1.0. This achieves a grid spacing (parameter resolution) of $1 \times 10^{-5}$ in $\Delta m_{32}^{2}$ and $1 \times 10^{-2}$ in $\sin ^{2} 2 \theta_{23}$. A nested loop is used to scan over every combination of $\Delta m_{32}^{2}$ and $\sin ^{2} 2 \theta_{23}$.

- An event loop is used within this grid scan to calculate the survival probability of each selected event for the current values of $\Delta m_{32}^{2}$ and $\sin ^{2} 2 \theta_{23}$. An analogous 2-D histogram in $P_{\text {reco }}$ versus $\cos \left(\theta_{\text {beam }}\right)$ is filled with each event, weighted by its survival probability.

- The predicted oscillated spectrum is scaled to the appropriate number of protons on target for the desired experiment.

- The expected number of events in each bin $i$ is compared to the observed number of events to form the fit $\chi^{2}$ for each point on the oscillation parameter grid space:

$$
\chi^{2}=-2 \mathcal{L}=-2 \sum_{i}\left[-N_{e x p}^{i}+N_{o b s}^{i}-N_{o b s}^{i} \ln \left(N_{o b s}^{i} / N_{e x p}^{i}\right)\right] .
$$

- This procedure is repeated for every point in the oscillation parameter space. The fit $\chi^{2}$ is stored as a 2-D surface in parameter space, along with the best-fit parameters.

In this manner, the best fit under the oscillation hypothesis is located for the given data or mock data Monte Carlo. See Appendix C.1 for the development of the log likelihood as used in this fit procedure.

\subsubsection{Fit with penalty terms for normalization}

Due to the uncertainty in the overall rock muon rate arising from both the modeling of the rock volume (see Section 3.2.3) in the GMINOS Monte Carlo and the $\mathrm{K} / \pi$ ratio uncertainty in the hadron production model, it is necessary to allow for an adjustment to the flux of high-energy muons at the Far Detector. These muons can reach the detector from deep within the upstream rock. Because of the higher average energy of neutrinos arising from kaon decay in the NuMI beam line (see Appendix B.2), it is desirable to 
separate the contributions of neutrinos parented by pions from those parented by kaons. This results in two independent template muon momentum distributions that can be normalized separately during the fit. Figure 6.2 displays the contribution of selected antifiducial muon events separated for pion and kaon parents (resulting from the $K / \pi$ ratio as modeled in GNuMI MC), on a log scale to emphasize the crossover point. Figure 6.3 shows the contributions on a normal scale to demonstrate the relative numbers present in the selected muon sample; approximately $30 \%$ of events below $30 \mathrm{GeV} / \mathrm{c}$ are parented by kaons, while only $\sim 20 \%$ below $10 \mathrm{GeV} / \mathrm{c}$ (the events used in the oscillation fit) arise from kaons.

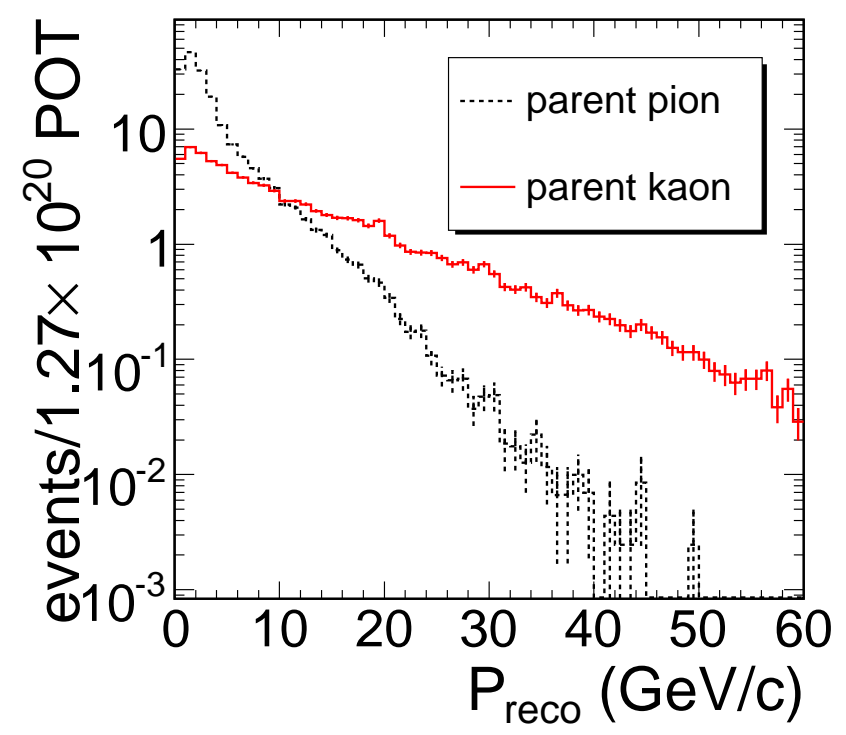

Figure 6.2: Selected Monte Carlo muon events below $60 \mathrm{GeV} / \mathrm{c}$, separated by those parented by pions and kaons. Pion contribution: dashed black line; kaon contribution: solid red line.

One can see the crossover point in Figure 6.2 around a reconstructed momentum of $10 \mathrm{GeV} / \mathrm{c}$, beyond which the kaon contribution starts to dominate the spectrum of selected muon events. This kaon component has the greatest uncertainty in absolute normalization, supporting the decision to perform the oscillation fit for events below $10 \mathrm{GeV} / \mathrm{c}$. 


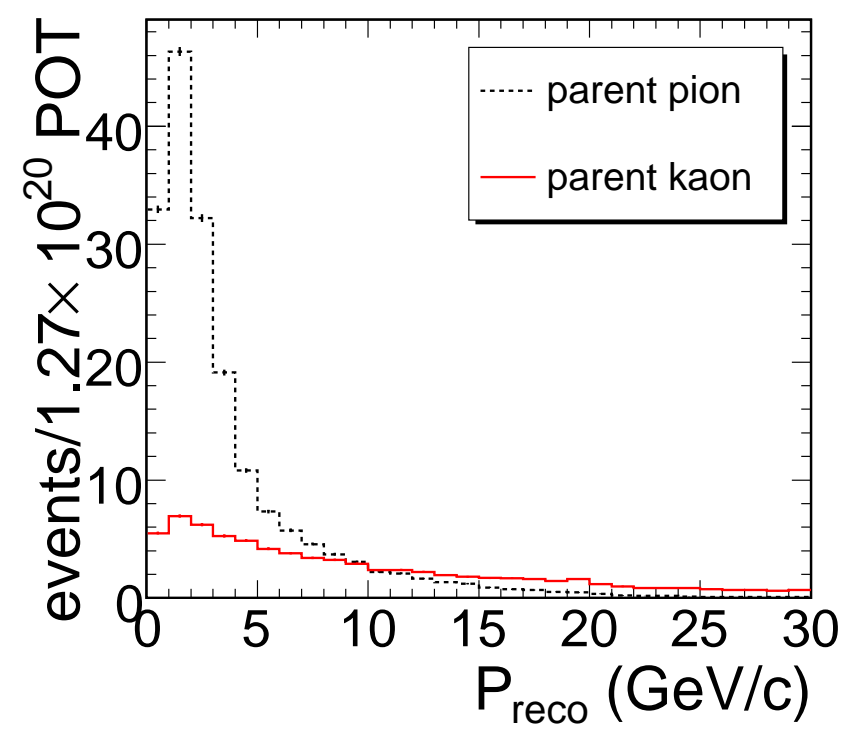

Figure 6.3: Selected Monte Carlo muon events below $30 \mathrm{GeV} / \mathrm{c}$, separated by those parented by pions and kaons. Pion contribution: dashed black line; kaon contribution: solid red line.

The neutrino spectrum giving rise to the antifiducial muon events can be separated in a similar manner to demonstrate the greater sensitivity to oscillation in the pion sample over the kaon sample. Figure 6.4 displays the true neutrino energies for muon events on a log scale to demonstrate the crossover point. Figure 6.5 shows the same on a normal scale to demonstrate the peak in the pion contribution in the region sensitive to the atmospheric neutrino oscillation parameters, while the kaon spectrum is very flat and quite low in the region sensitive to oscillations. These two figures utilize all selection cuts except the momentum and angle cuts, to display the full spectrum. Figures 6.6 and 6.7 include the momentum and direction cuts used for the oscillation fit. These emphasize the lower energy regions of the neutrino spectrum that are most sensitive to oscillation. The kaon contribution is further suppressed, while the pion peak remains.

The fit procedure described above is repeated, with a nested loop over a grid of normalization parameters $N_{\pi}$ and $N_{K}$ with a resolution of $1 \%$ on each parameter, both allowed to float within $\pm 3 \sigma$ of their nominal values. To account for our knowledge of the 


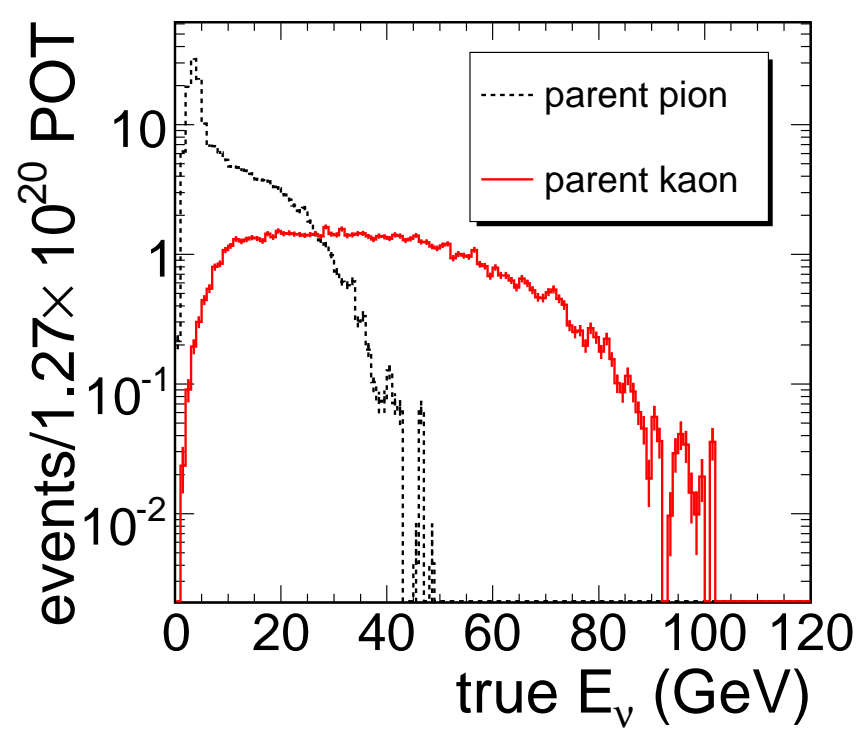

Figure 6.4: Parent neutrino spectra below $120 \mathrm{GeV}$ for muon events selected (up to momentum and direction cuts), separated by those parented by pions and kaons. Pion contribution: dashed black line; kaon contribution: solid red line.

uncertainty on these parameters, "penalty terms" are added to the fit $\chi^{2}$ :

$$
\chi^{2}=\sum_{i}\left[-N_{e x p}^{i}+N_{o b s}^{i}-N_{o b s}^{i} \ln \left(N_{o b s}^{i} / N_{e x p}^{i}\right)\right]+\frac{\Delta N_{\pi}}{\sigma_{N_{\pi}}}+\frac{\Delta N_{K}}{\sigma_{N_{K}}},
$$

where $\Delta N_{\pi, K}$ represents the shift in the normalization (away from 1.0) preferred by the systematic best fit for the pion or kaon normalization, and $\sigma_{N_{\pi, K}}$ represents the uncertainty assigned to those normalizations: $\sigma_{N_{\pi}}=0.04$ and $\sigma_{N_{K}}=0.20$. The additional systematic terms in the fit $\chi^{2}$ are called penalty terms because a shift in one of the normalization parameters by one sigma "penalizes" the minimum chi-squared value by $\Delta \chi^{2}=1.0$. In this fit, the normalization terms multiply the number of expected events in each bin according to their truth parent hadron, so that the $N_{\text {exp }}$ in Equation 6.2 are understood to contain this weighting factor: $N_{e x p}=\sigma_{N_{\pi}} N_{e x p}^{\pi}\left(\Delta N_{\pi}=0\right)+\sigma_{N_{K}} N_{e x p}^{K}\left(\Delta N_{K}=0\right)$.

The motivation for the pion uncertainty of $4 \%$ comes from the relative near to far normalization uncertainty, as reported in Reference [1] and detailed in Reference [65]. One major uncertainty contributing here is our knowledge of the fiducial mass ratio 


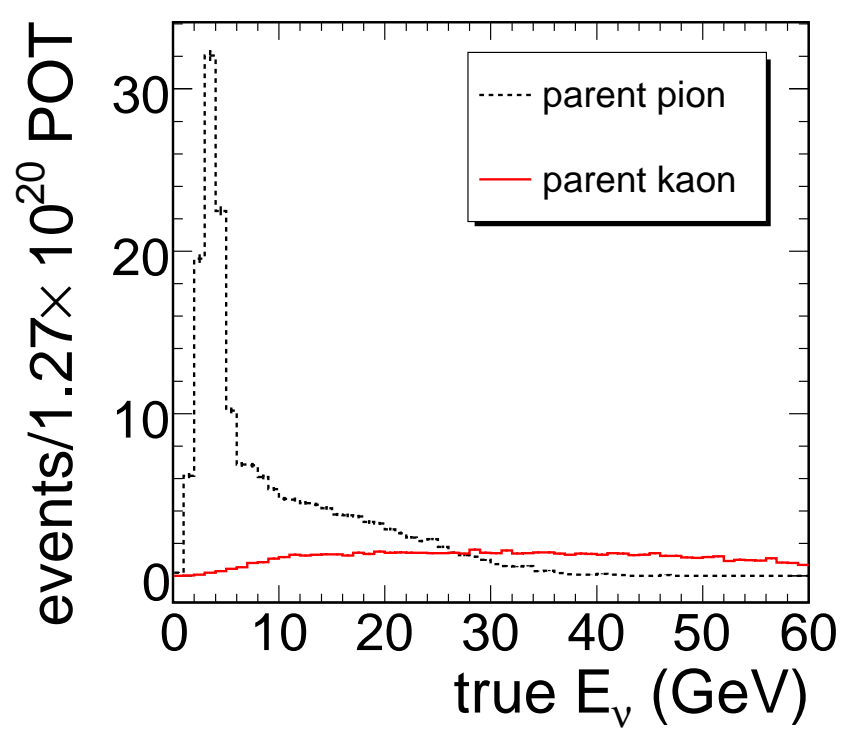

Figure 6.5: Parent neutrino spectra below $60 \mathrm{GeV}$ for muon events selected (up to momentum and direction cuts), separated by those parented by pions and kaons. Pion contribution: dashed black line; kaon contribution: solid red line.

$M_{f i d}^{F D} / M_{f i d}^{N D}$, which is uncertain at the $2 \%$ level. The other main contributions include the absolute POT counting uncertainty in the beam line monitors (1\%) and relative differences between data and Monte Carlo reconstruction inefficiencies (3\%).

For the kaon uncertainty, a value of $20 \%$ is adopted. This is the reported uncertainty in the $K / \pi$ ratio used by the NuMI beam systematics working group[55] to generate their SKZP fit to align the Near Detector MC with the reconstructed data. This normalization uncertainty should be understood to allow correction for both the kaon flux in GNuMI and the muon events arising from the deficient outer layer of rock in the Far Detector GMINOS model. 


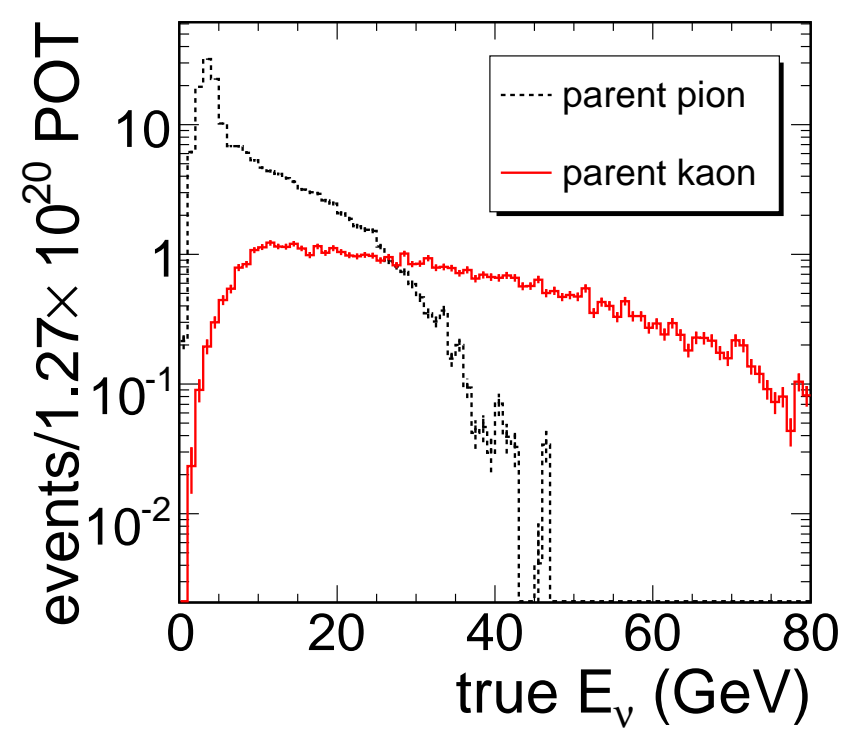

Figure 6.6: Parent neutrino spectra below $80 \mathrm{GeV}$ for muon events passing all selection cuts, separated by those parented by pions and kaons. Pion contribution: dashed black line; kaon contribution: solid red line.

\subsection{Mock data}

Two sets of "mock data" Monte Carlo were generated by R. Hatcher for the purpose of testing analysis methods, corresponding to $\sim 100 \times 10^{20}$ POT each. He chose input oscillation parameters (identical for both sets) which remained unknown to those performing analyses on the mock data. The first set used the standard GNuMI neutrino flux, while the second set used a "tweaked" flux that mimicked possible distortions in the spectrum that could arise in the data.

Here the fit results are presented for both samples, utilizing the entire available sample and scaling down the exposure to $1.27 \times 10^{20}$ POT . Statistical uncertainties are quoted on the measured parameters and confidence level contours resulting from the fits are displayed. 


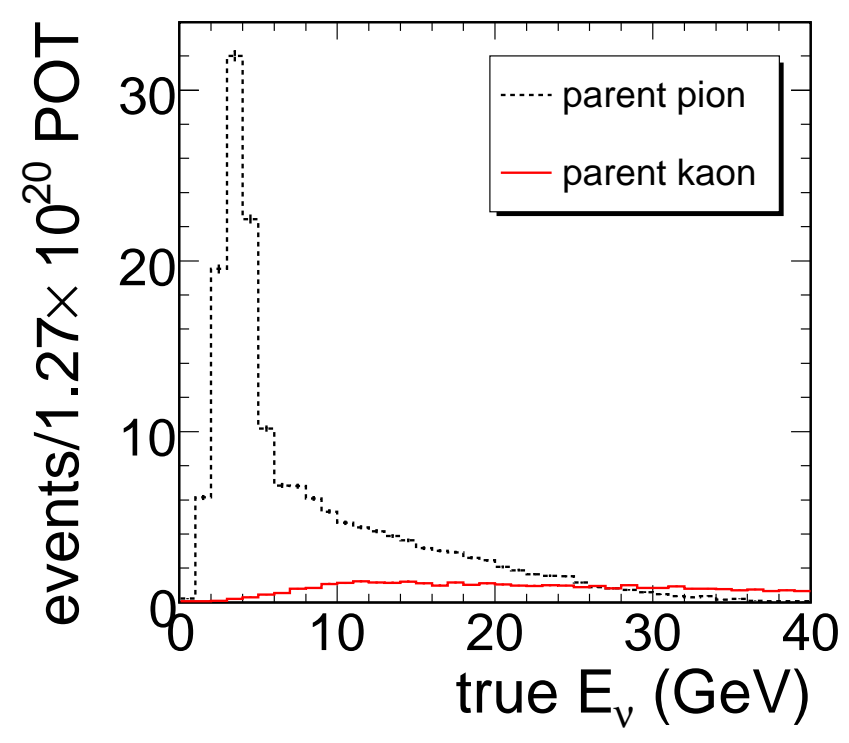

Figure 6.7: Parent neutrino spectra below $40 \mathrm{GeV}$ for muon events passing all selection cuts, separated by those parented by pions and kaons. Pion contribution: dashed black line; kaon contribution: solid red line.

\subsection{Normal flux}

Given a sample of contained-vertex and rock-vertex Monte Carlo with input oscillation parameters $\left|\Delta m_{32}^{2}\right|=2.38 \times 10^{-3} \mathrm{eV}^{2} / \mathrm{c}^{4}$ and $\sin ^{2} 2 \theta_{23}=0.93$, the fit performs quite well, extracting parameters $\left|\Delta m_{32}^{2}\right|=2.30 \times 10^{-3} \mathrm{eV}^{2} / \mathrm{c}^{4}$ and $\sin ^{2} 2 \theta_{23}=0.94$. This MC sample was just over $100 \times 10^{20}$ POT and utilized the standard V.18 flux files (see Section 3.1). Figure 6.8 displays three confidence level contours for the $1.27 \times 10^{20} \mathrm{POT}$ equivalent sensitivity.

The measured parameters with statistical uncertainties are:

$$
\left|\Delta m_{32}^{2}\right|=2.30 \pm_{0.60}^{5.10} \times 10^{-3} \mathrm{eV}^{2} / \mathrm{c}^{4} \text { and } \sin ^{2} 2 \theta_{23}>0.31 \text { at } 68 \% \text { C.L. }
$$

The degeneracy of the two parameters is evident here, causing the allowed regions to balloon out to lower $\sin ^{2} 2 \theta_{23}$ and higher $\left|\Delta m_{32}^{2}\right|$. The "banana"-shaped contours are accentuated with an input mixing angle away from maximal mixing. Ultimately, the fit performs as expected, returning a value $3 \%$ low in $\left|\Delta m_{32}^{2}\right|$ and $1 \%$ high in $\sin ^{2} 2 \theta_{23}$. 


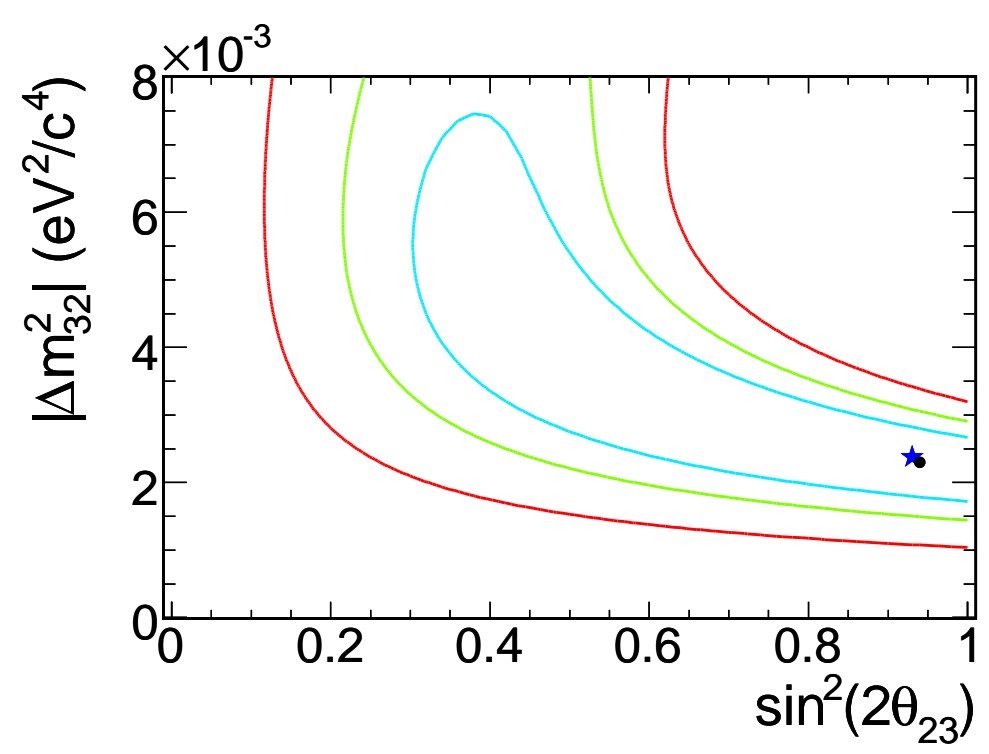

Figure 6.8: Contours for mock data, normal flux. Light blue (inner), $\Delta \chi^{2}=1.0$; green (middle), 68\% C.L.; red (outer), 90\% C.L. The input parameters are shown with a blue star, the best fit with a black dot.

\subsection{Tweaked flux}

Given a sample of contained-vertex and rock-vertex Monte Carlo with input oscillation parameters $\left|\Delta m_{32}^{2}\right|=2.38 \times 10^{-3} \mathrm{eV}^{2} / \mathrm{c}^{4}$ and $\sin ^{2} 2 \theta_{23}=0.93$, the fit performs as expected, extracting parameters $\left|\Delta m_{32}^{2}\right|=3.48 \times 10^{-3} \mathrm{eV}^{2} / \mathrm{c}^{4}$ and $\sin ^{2} 2 \theta_{23}=0.60$. This MC sample was just under $100 \times 10^{20}$ POT and utilized modified versions of the standard V.18 flux files. The modifications were generated in a manner consistent with large shifts in the SKZP beam fit[55] parameters: the horn position, current distribution and magnitude were all shifted up to $50 \%$ of their nominal values. Hadron production parameters were also modified to give a different beam flux than in the nominal Monte Carlo. Figure 6.9 shows the original beam flux as modeled in the Near Detector MC alongside the modified spectrum used to generate this mock data sample. This modified ND spectrum was provided so that it could be used in the extrapolation to the FD. The ultimate effect of the beam tweak was to increase the event rate in the focusing peak of the neutrino spectrum. Figure 6.8 displays three confidence level contours for the 
$1.27 \times 10^{20}$ POT equivalent sensitivity.

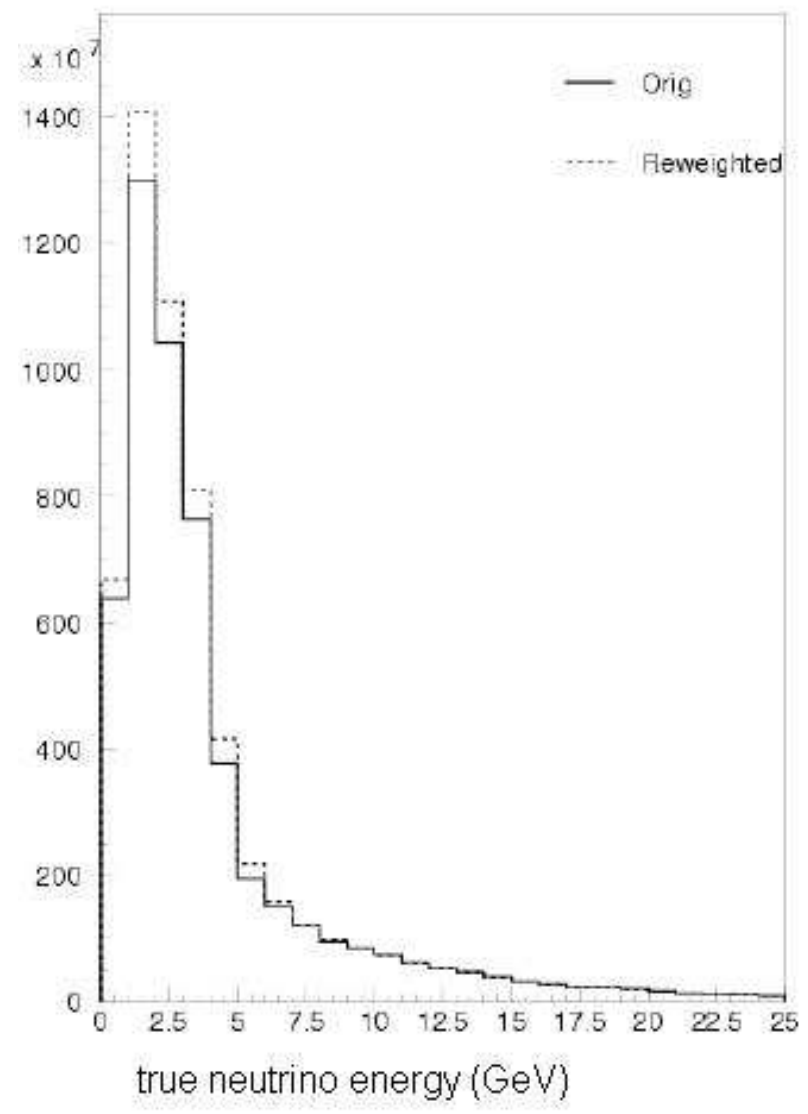

Figure 6.9: Modified Near Detector neutrino spectrum as used in the Mock Data samples. Plot taken from Marino[66]. Solid line: original true neutrino energy distribution; dashed line: modified distribution.

The measured parameters with statistical uncertainties are:

$$
\left|\Delta m_{32}^{2}\right|=3.48 \pm_{1.58}^{4.42} \times 10^{-3} \mathrm{eV}^{2} / \mathrm{c}^{4} \text { and } \sin ^{2} 2 \theta_{23}>0.31 \text { at } 68 \% \text { C.L. }
$$

The degeneracy of the two parameters this time shifts the best fit along the trough of the $\chi^{2}$ valley. Ultimately, the fit performs as expected, returning a value $3 \%$ low in $\left|\Delta m_{32}^{2}\right|$ and $1 \%$ high in $\sin ^{2} 2 \theta_{23}$. 


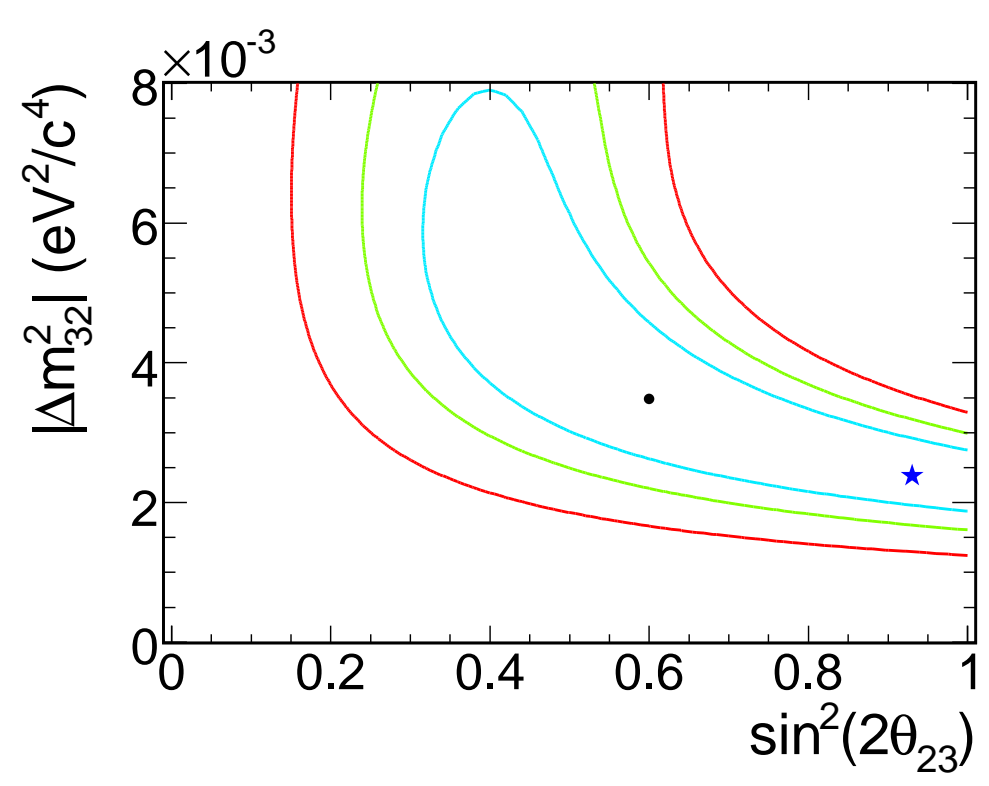

Figure 6.10: Contours for mock data, tweaked flux. Light blue (inner), $\Delta \chi^{2}=1.0$; green (middle), 68\% C.L.; red (outer), 90\% C.L. The input parameters are shown with a blue star, the best fit with a black dot.

\subsection{Precision of fit parameter measurements}

At the current exposure of $1.27 \times 10^{20}$ POT , the precision to which the oscillation parameters can be measured is affected by the true value of the parameters. In general, the oscillation probability (and thus the size of the measured deficit) is linearly proportional to the magnitude of $\sin ^{2} 2 \theta_{23}$ and quadratically proportional to the magnitude of $\left|\Delta m_{32}^{2}\right|$. The preponderance of experimental evidence for maximal mixing led me to fix $\sin ^{2} 2 \theta_{23}=1.0$ and test different values of $\left|\Delta m_{32}^{2}\right|$. Because the deficit increases approximately as $\left(\Delta m_{32}^{2}\right)^{2}$ and the statistical uncertainty decreases as the square root of the measured deficit, one expects to see a nearly linear decrease in the uncertainty (increase in precision) on the measured value as $\left|\Delta m_{32}^{2}\right|$ increases. Figure 6.11 demonstrates this effect using the $68 \%$ confidence level contours at an exposure of $3.0 \times 10^{20}$ POT.

Further studies of the sensitivity of the rock muon sample using GMINOS Monte Carlo, performed by the author and G. Giurgiu, can be found in Reference [67]. At the time, the analysis was focused on using rock muons only, and the study of contained 


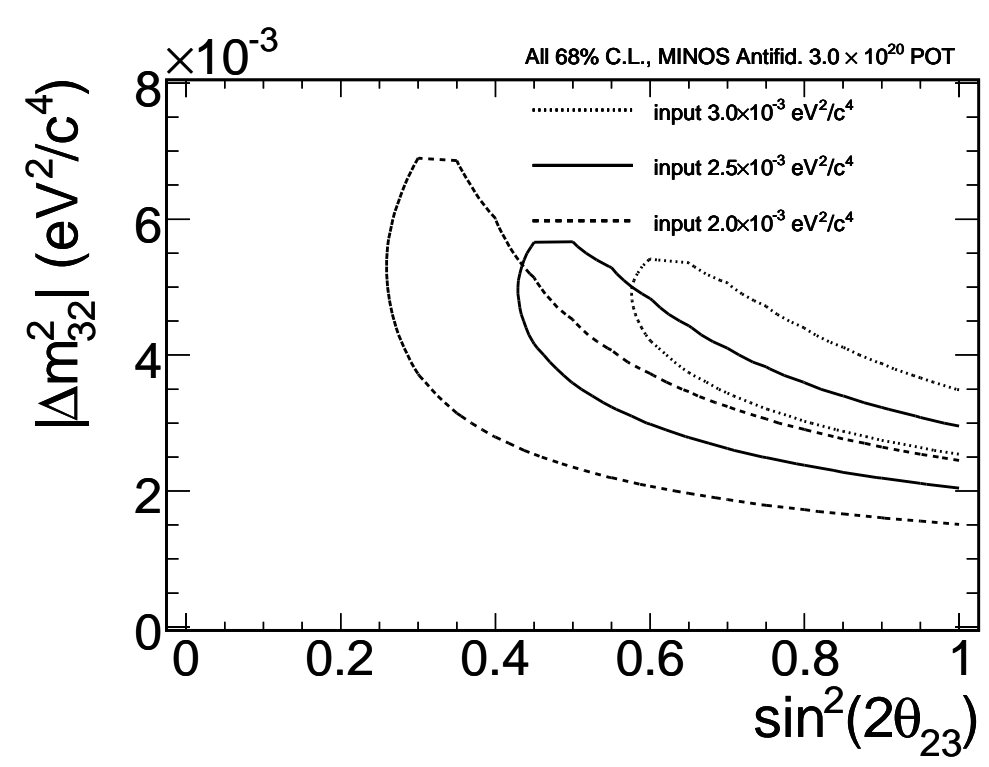

Figure 6.11: $68 \%$ CL contours at an exposure of $3.0 \times 10^{20}$ POT for three different input oscillation parameters. The statistical uncertainty on the measurement decreases linearly with $\left|\Delta m_{32}^{2}\right|$.

vertex events passing the rock muon selection cuts prompted further investigation into an analysis that utilized both event samples to their maximal potential.

When maximal mixing is imposed at an input value of $\left|\Delta m_{32}^{2}\right|=2.0 \times 10^{-3} \mathrm{eV}^{2} / \mathrm{c}^{4}$, a $15 \%$ measurement of $\left|\Delta m_{32}^{2}\right|$ is possible. At $\left|\Delta m_{32}^{2}\right|=2.5 \times 10^{-3} \mathrm{eV}^{2} / \mathrm{c}^{4}$, this improves to $12 \%$. And at $\left|\Delta m_{32}^{2}\right|=3.0 \times 10^{-3} \mathrm{eV}^{2} / \mathrm{c}^{4}$, the precision improves to $10 \%$.

Because changes in one parameter can mimic changes in the other parameter, there is a degeneracy between the oscillation parameters present in this measurement. The upward slope of the confidence level contours in Figure 6.11 indicates a negative correlation between the parameters: very similar measured distributions can be achieved by increasing $\left|\Delta m_{32}^{2}\right|$ and decreasing $\sin ^{2} 2 \theta_{23}$, or vice versa. At an exposure of $3.0 \times 10^{20}$ POT, this correlation still plagues the analysis; however, the shape of the contours becomes much more elliptical and the correlation (slope of the ellipse) decreases at higher exposures.

While we have no control over the true values of the oscillation parameters in real life, we can increase the precision of our measurement by running the experiment for a longer 
duration - the statistical uncertainty on the probability of oscillation decreases as the square root of the size of the deficit, which is proportional to $\sin ^{2} 2 \theta_{23}$ or $\left(\Delta m_{32}^{2}\right)^{2}$ as mentioned previously. After two years of NuMI running, we have accumulated approximately $3.0 \times 10^{20}$ protons on target in the standard low-energy configuration. Planned upgrades will increase the intensity of proton pulses on the NuMI target as well as decrease the length of time between pulses, increasing the delivered power to the target. These upgrades will increase our rate of event accumulation, shortening the doubling times in years to come. See Figure 7.17 for expected MINOS antifiducial analysis sensitivities for three representative exposures of the NuMI target.

\subsection{Systematic uncertainties}

Various systematic effects have been investigated for assumed oscillation parameters $\Delta m_{32}^{2}=2.74 \times 10^{-3} \mathrm{eV}^{2} / \mathrm{c}^{4}$ and $\sin ^{2} 2 \theta_{23}=1.0$. By shifting a systematic parameter at the Near Detector, extrapolating the resulting spectrum to the Far Detector, and comparing with the expected Monte Carlo distribution at the FD (using the same shift for this expected distribution), an estimate of the effect can be obtained by measuring the shift in $\left|\Delta m_{32}^{2}\right|$ and $\sin ^{2} 2 \theta_{23}$. The major systematic uncertainties and their expected levels are summarized in Table 6.2.

\subsubsection{Normalization}

The overall normalization of the neutrino flux arising from parent pions is shifted by $\pm 4 \%$. This uncertainty is driven by the same uncertainty on the fiducial containedvertex analysis (see Section 6.1.2). The overall normalization of the neutrino flux arising from parent kaons is shifted by $\pm 20 \%$. This value was chosen as a combined estimate of the uncertainty arising from the volume used in the GMINOS rock model and the $K / \pi$ ratio in the GNuMI beam model. The absolute event rate arising from kaons, while mitigated by the decision to fit only events up to $10 \mathrm{GeV} / \mathrm{c}$, still represents the largest systematic uncertainty for the analysis.

\subsubsection{Cross sections}

Due to uncertainty in the Soudan rock composition upstream of the Far Detector, errors in knowledge of the neutrino cross section contribute to the uncertainty in the muon 
event rate. The rock composition as modeled in GMINOS and as measured in surveys is discussed in Section 3.3. A conservative estimate of $20 \%$ is adopted as the cross section uncertainty for this effect. The cross sections in the GMINOS model for deep-inelastic scattering, quasi-elastic scattering, and pion production are shifted one at a time by $\pm 20 \%$ for events in the rock. After the shift in the "test data" sample, the oscillation fit is performed and the effect of each shift is recorded. Events with a neutrino interaction in the detector are not affected, since the cross section uncertainties cancel between the Near and Far Detectors. The individual cross section effects (as an average of the $\pm 20 \%$ shifts) are listed in Table 6.1. The reported total cross section uncertainty is given as the quadrature sum of the DIS, QE, and pion production (RES) cross section effects.

\begin{tabular}{c|c|c|c|c}
\hline \hline Effect & $\delta\left(\left|\Delta m_{32}^{2}\right|\right)\left(\mathrm{eV}^{2} / \mathrm{c}^{4}\right)$ & $\delta\left(\sin ^{2} 2 \theta_{23}\right)$ & events $<10 \mathrm{GeV} / \mathrm{c}$ & events $<3 \mathrm{GeV} / \mathrm{c}$ \\
\hline QE & $1.3 \mathrm{e}-4$ & $0.5 \mathrm{e}-1$ & 4.7 & 3.5 \\
RES & $2.0 \mathrm{e}-4$ & $0.5 \mathrm{e}-1$ & 7.5 & 5.3 \\
DIS & $0.6 \mathrm{e}-4$ & $0.3 \mathrm{e}-1$ & 2.8 & 2.0 \\
\hline Total & $2.5 \mathrm{e}-4$ & $0.8 \mathrm{e}-1$ & 9.3 & 6.7 \\
\hline \hline
\end{tabular}

Table 6.1: Summary of cross section uncertainties and their effect on the fit parameters and the total expected event rates.

\subsubsection{Muon energy loss}

Another effect of the rock composition uncertainty is its impact on the muon $d E / d X$ values. It has been demonstrated (in Section 3.2.2) that the ionization energy loss term is the only relevant mechanism at the muon energies arising from NuMI beam interactions, and that the average $Z / A$ value determines the ionization energy loss for muons. As seen in Section 3.3, the difference between $Z / A$ for Soudan rock and for the rock as modeled in GMINOS is only $1 \%$. Therefore, a conservative estimate is taken that the uncertainty in the Soudan rock composition is equal to the difference between the survey measurements and the GMINOS model. The $Z / A$ value is shifted by $\pm 1 \%$, and the test data is fit to record the effect on the oscillation parameters.

\subsubsection{Neutral current background}

The magnitude of the neutral current background rate is affected by our uncertainty of the total NC cross section. To account for this, the NC component of the test data distribution is shifted by $\pm 50 \%$. While the nominal number of $\mathrm{NC}$ events passing selection 
is only 4.1 out of 252.9 , these events fall in the corner of the reconstructed momentum/direction space most sensitive to the oscillation deficit (the peak shown in Figure 7.9). The $50 \%$ decrease in these events has very little effect on the measured parameters, but the $50 \%$ increase has a substantial effect at this level of statistics and is one of the major contributors to the overall uncertainty.

\subsubsection{Track momentum and angle}

Biases between data and Monte Carlo in our momentum measurement of tracks in the MINOS Far Detector lead to a significant uncertainty for this analysis, which relies heavily on the determination of the muon momentum distribution. Because our knowledge of muon energy loss in steel is more precise than our ability to measure momentum from curvature from exiting tracks (at the low beam energies involved, multiple scattering in the steel planes can scramble curvature information), momentum measured from range carries a smaller uncertainty. The reconstructed track momentum is shifted by $\pm 6 \%$ for events with momentum measured from curvature; it is shifted by $\pm 2 \%$ for events with momentum measured from range. A similar study on the reconstructed track angle concluded that the uncertainty on this quantity (on the order of $1 \%$ ) caused a negligible shift on the fit oscillation parameters.

\subsubsection{Summary}

The uncertainties discussed here are each considered separately as follows, with the reported effect for $\left|\Delta m_{32}^{2}\right|$ given as the average effect of shifting the parameter up and down by the stated amount. Since the physical boundary is imposed on all fits (causing any fit that prefers a non-physical solution to lie on the boundary), the reported effect for $\sin ^{2} 2 \theta_{23}$ is the deviation from 1.0 if the other shift returns 1.0 for the best fit; if both shifts reduce $\sin ^{2} 2 \theta_{23}$, the average is reported. The total systematic uncertainty is the quadrature sum of each effect.

The ultimate effect of each parameter shift (besides the track momentum uncertainty, which shifts the distribution left/right) is to shift the normalization up/down. Most shape information that would be present in the neutrino energy spectrum is washed out, due to the unknown interaction vertex in the rock and the unknown fraction of the neutrino energy carried by the muon. The two normalization parameters $N_{K}$ and $N_{\pi}$ are optimized during the fit to the oscillation parameters $\left|\Delta m_{32}^{2}\right|$ and $\sin ^{2} 2 \theta_{23}$. 


\begin{tabular}{c|c|c|c|c|c}
\hline \hline Effect & shift & $\delta\left(\left|\Delta m_{32}^{2}\right|\right)\left(\mathrm{eV}^{2} / \mathrm{c}^{4}\right)$ & $\delta\left(\sin ^{2} 2 \theta_{23}\right)$ & $<10 \mathrm{GeV} / \mathrm{c}$ & $<3 \mathrm{GeV} / \mathrm{c}$ \\
\hline$N_{\pi}$ & $4 \%$ & $2.2 \mathrm{e}-4$ & $0.7 \mathrm{e}-1$ & 7.7 & 5.1 \\
$N_{K}$ & $20 \%$ & $3.9 \mathrm{e}-4$ & $2.1 \mathrm{e}-1$ & 11.9 & 4.8 \\
NC & $50 \%$ & $2.5 \mathrm{e}-4$ & $2.5 \mathrm{e}-1$ & 2.1 & 2.0 \\
cross section & $20 \%$ & $2.5 \mathrm{e}-4$ & $0.8 \mathrm{e}-1$ & 9.3 & 6.7 \\
rock comp. & $1 \%$ & $0.8 \mathrm{e}-4$ & $0.1 \mathrm{e}-1$ & 4.1 & 2.4 \\
$P_{\text {reco }}$ & $2 \%, 6 \%$ & $2.1 \mathrm{e}-4$ & $1.5 \mathrm{e}-1$ & 1.2 & 2.6 \\
\hline Total & & $6.1 \mathrm{e}-4$ & $3.7 \mathrm{e}-1$ & 17.6 & 10.5 \\
\hline \hline
\end{tabular}

Table 6.2: Summary of systematic uncertainties and their effect on the fit parameters and the total expected event rates. The total effect on each parameter is the sum in quadrature of the individual effects. 


\section{Chapter 7}

\section{Observed data and fit results}

I like to think the moon is there even if I am not looking at it.

-Albert Einstein

Various consistency checks were carried out when this analysis received approval for box-opening. All selected events were re-scanned by hand to ensure there were no obvious pathologies in the sample. The arrival times of the events were scrutinized to fall within the $10 \mu$ s beam spill window. Comparisons of important reconstructed variables between the selected data and the Monte Carlo expectation were carried out. At the end of this chapter, the hypothesis test used in this analysis to rule out the no-disappearance case is described.

\subsection{Event arrival time}

The arrival time of each event was compared with the expected arrival time of the first neutrino from the NuMI beam spill window. As described in Section 4.2.1, the arrival time is calculated using two GPS timestamps, measured relative to the same time origin. The first is the expected arrival time fartdb of the earliest neutrino from the beam spill, found by adding the transit time to the Far Detector for relativistic neutrinos $(2.45 \mathrm{~ms})$ to the GPS time stamp of the spill window opening in the NuMI beam line at Fermilab. The second is the FD snarl start time snarlt. The snarl begins when any trigger is set (see Section 2.4.4), usually the spill trigger. The time of the earliest hit in the event, evttimemin, is recorded relative to snarlt. By adding this hit time to the snarl time, then subtracting the expected arrival time of the beam spill, we arrive at the desired 
arrival time variable snarlt - fartdb + evttimemin. Figure 7.1 shows all 232 selected data events falling within the $10 \mu$ s beam spill window, as well as one atmospheric muon event (removed by the timing cut) that falls in the sideband. With 0.38 events expected in this $40 \mu \mathrm{s}$ sideband (see Section 4.5.1) the Poisson probability for observing one event is $26 \%$. Figure 7.2 shows a more detailed binning for the $10 \mu$ s beam spill window, revealing some semblance of the NuMI batch structure. With more statistics, this plot will begin to resemble Figure 2.5, the event timing distribution at the MINOS Near Detector.

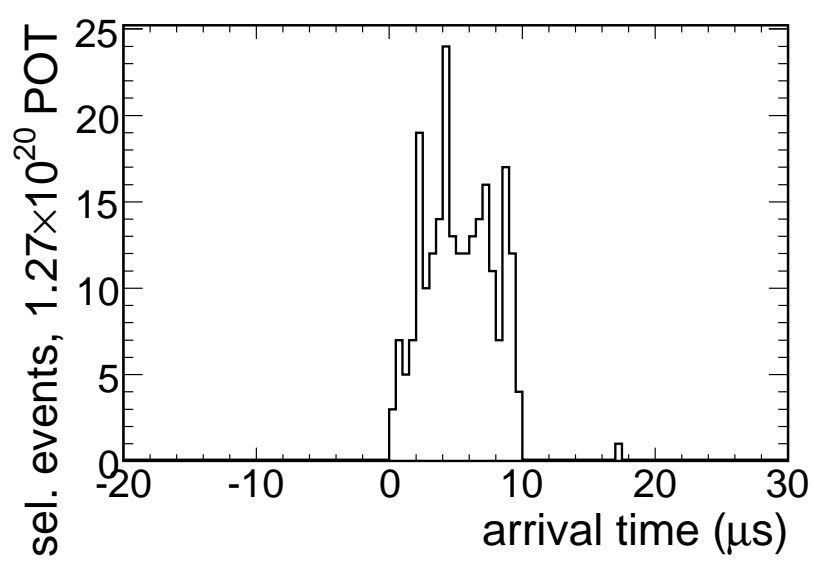

Figure 7.1: Arrival time of selected data events with respect to the expected arrival time of the first neutrino from each relevant NuMI beam spill. The sideband is displayed for $\pm 20 \mu$ s around the beam spill window, demonstrating one cosmic ray muon where 0.4 events are expected.

\subsection{Comparison of reconstructed data to $\mathrm{MC}$}

Before performing an oscillation fit to the selected data, various reconstructed event quantities were compared to their Monte Carlo expectations. The categories shown here are reconstructed track vertex and endpoint location, track and shower reconstruction consistency, momentum distributions, and the PID parameter described in Section 4.3. In these discussions, the focus is placed on the rock muon contribution to the distributions, 


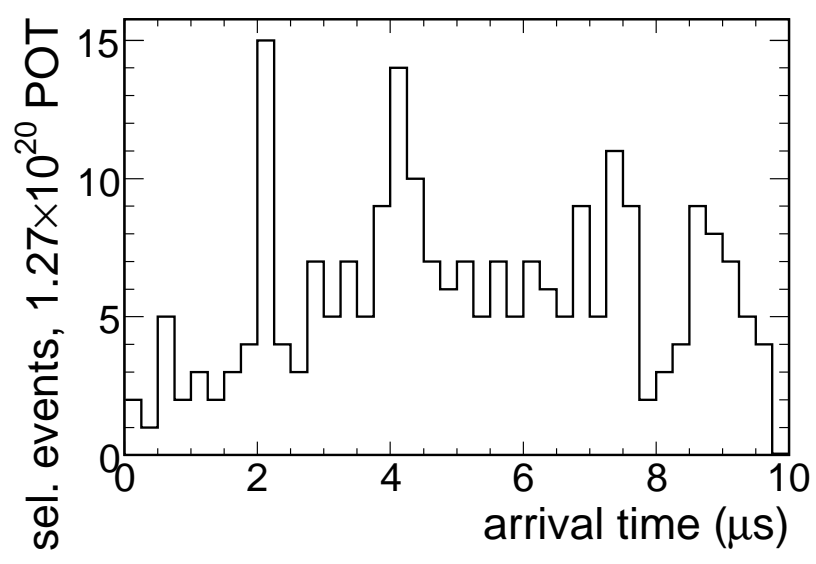

Figure 7.2: Zoomed arrival time of selected data events with respect to the expected arrival time of the first neutrino from each relevant NuMI beam spill. With the $0.25 \mu \mathrm{s}$ binning, the NuMI batch structure is starting to appear at this POT exposure.

as they are responsible for the majority of selected events. Bear in mind that containedvertex neutrino interactions are also present in these distributions, and that they are expected to be distributed uniformly throughout the antifiducial volume.

\subsubsection{Track vertex and endpoint location}

The reconstruction of neutrino and muon interactions in the MINOS Far Detector assigns a location $(x, y, z)$ to the vertex and endpoint of the track. These positions are based on the raw hit information, which arrives in the $(u, v, z)$ coordinate system. Demonstrating agreement between these distributions in both the raw coordinates and the reconstructed coordinates between data and Monte Carlo gives confidence that the neutrino interactions in the detector and surrounding rock are well-modeled in GMINOS. In the following comparison plots, all selected data events (obtained by excluding the $10 \mathrm{GeV} / \mathrm{c}$ cut on muon momentum, used only for the oscillation fit) are shown as black points with statistical error bars, and the MC expectation arising from the extrapolation (see Chapter 5 ) is shown as a solid blue histogram.

Figure 7.3 shows the reconstructed $z$ and $r^{2}=x^{2}+y^{2}$ positions for both the track vertices and endpoints. The two left plots nicely demonstrate the two classes of rock 
muon events. In the vertex z-position plot, a large peak in the first bin is seen. This represents the muons penetrating the front surface of the detector. The flat distribution beyond the front peak at larger values of $z$ represents those events penetrating all sides of the detector. The SM gap does not appear in this plot due to the $2 \mathrm{~m}$ bin size. In the vertex radius-squared plot, another peak is seen, corresponding to large values of the vertex radius. Such events are the side-entry rock muons from the flat distribution in the previous plot. Here we also see a flat distribution at smaller values of $r^{2}$, corresponding to the peak at the front of the detector in the previous plot.

The two right plots in Figure 7.3 show the corresponding quantities for the track endpoints. The gap between SuperModules can be seen here due to the $1 \mathrm{~m}$ bin size. Since the detector can be approximated as an extension of the surrounding rock, one might expect to see equal numbers of muons exiting the rear of the detector as entering the front of the detector. Accounting for the finer binning (twice the bins as in the track vertex $z$ plot) we observe about one-third of the number exiting the rear as enter the front of the detector. The larger spread can be understood when the initial muon direction distribution is taken into account. While the forward-field (normal configuration) current generates a magnetic field that focuses $\mu^{-}$toward the center of the detector, many muons have an initial momentum that results in a trajectory that leaves the side face of the detector. In the endpoint radius-squared plot, the focusing effect is quite evident, pulling most muons close to the center of the detector before they range out. The first bin includes those events which terminate inside of the coil hole.

Figure 7.4 shows the detailed track vertex positions in raw transverse coordinates $u$ and $v$ as well as reconstructed coordinates $x$ and $y$. We expect to see a distribution that is symmetric in $x$, since the detector z-axis lies in the yz-plane (the detector is not rotated about the $\mathrm{y}$-axis). However, we expect asymmetries in the other three vertex position plots. Due to the $\sim 3^{\circ}$ upslope of the beam with respect to the local vertical (y-axis), we expect to see more muons entering the bottom of the detector $(y=-4 \mathrm{~m})$ than entering the top $(y=+4 \mathrm{~m})$. Since the uv-plane is rotated $45^{\circ}$ counterclockwise from the xy-plane, the excess of events at the bottom of the detector manifests as an excess at both the $u=-4 \mathrm{~m}$ and the $v=-4 \mathrm{~m}$ side faces; these excesses should appear equally distributed in the two orthogonal planes, as the u-axis and v-axis lie symmetric to the $\mathrm{y}$-axis.

Figure 7.5 similarly shows the detailed track endpoint positions in raw transverse coordinates $u$ and $v$ as well as reconstructed coordinates $x$ and $y$. Here, the asymmetries 

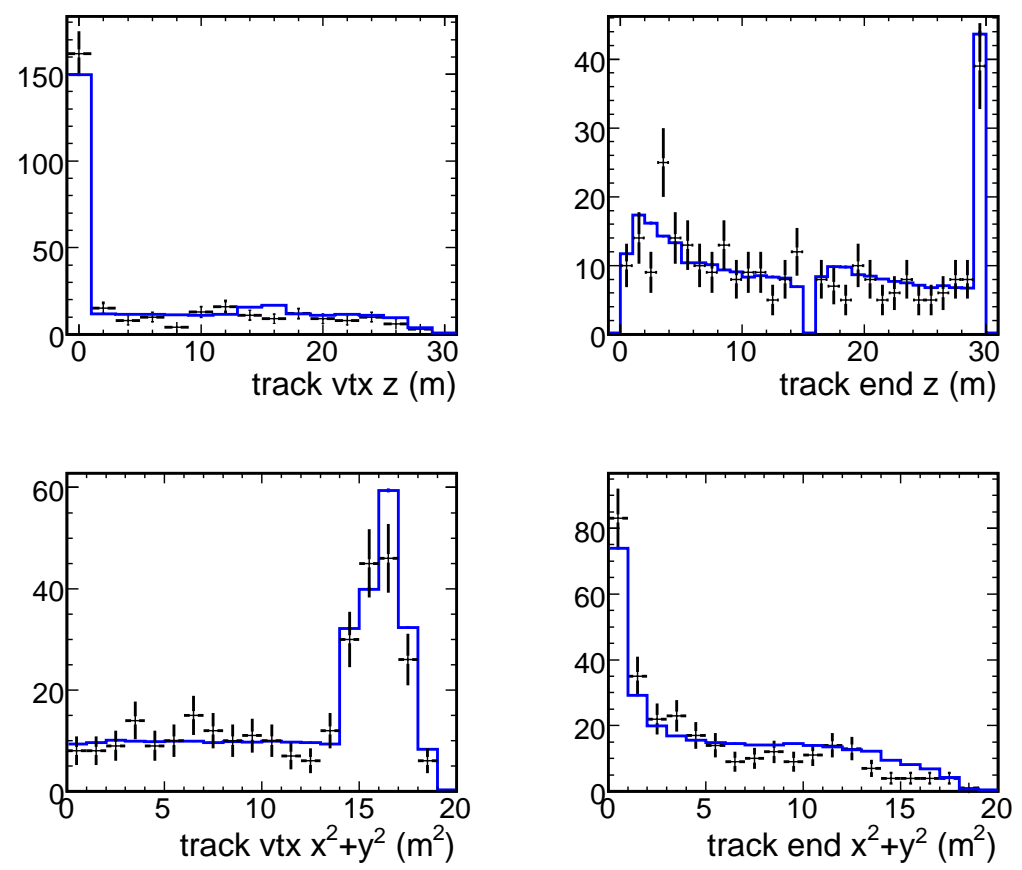

Figure 7.3: Reconstructed track vertex and endpoint locations. Top left: track vertex location in z. Top right: track endpoint location in z. Bottom left: track vertex location in $r^{2}=x^{2}+y^{2}$. Bottom right: track endpoint location in $r^{2}=x^{2}+y^{2}$.

observed in the track vertex plots are essentially wiped out by the strong focusing effect of the magnetic field. As expected, we see a grouping of events that terminate in or around the coil hole, where the magnetic field is the strongest. In all coordinates $u, v$, $x$, and $y$, the center of the detector lies at the origin.

\subsubsection{Track and shower fitting performance}

Figure 7.6 displays various tests of the track and shower modeling. Both "track fit pass" (upper left) and "track $\chi^{2} / \mathrm{N}_{d o f}$ " (lower right) are measures of the agreement between raw hits assigned to the track and the actual trajectory that is fit to these hits. The small percentage of tracks that do not pass the basic fit test are a result of those muons which range out in the detector and have a good momentum determination from range, but which had a poor fit to the curvature of the track hits. This results mainly from 

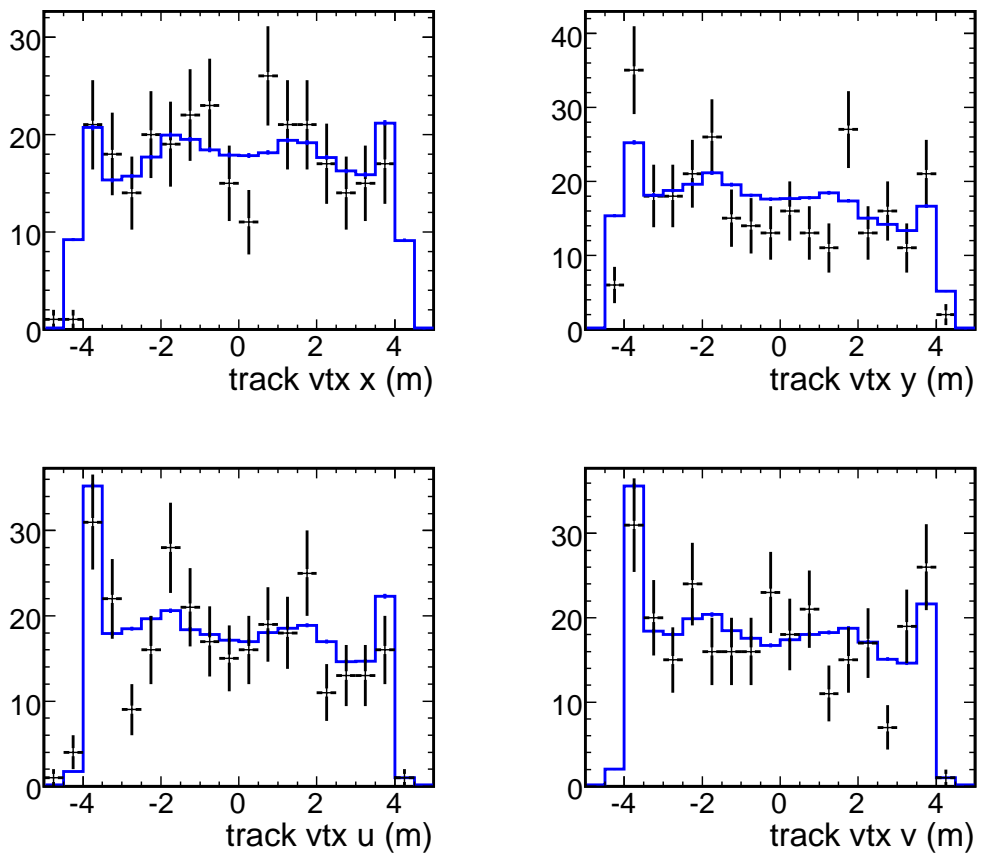

Figure 7.4: Reconstructed track vertex locations. Top left: track vertex location in $\mathrm{x}$. Top right: track vertex location in y. Bottom left: track vertex location in $\mathrm{u}$. Bottom right: track vertex location in $\mathrm{v}$.

reconstruction problems that occur at roughly the same rate in the data and Monte Carlo. The track $\chi^{2} / \mathrm{N}_{\text {dof }}$ is calculated from the deviation of the individual track hits from the fit trajectory. This plot demonstrates a peak at $\chi^{2}=1.0$, which is the usual behavior for a chi-squared distribution such as this, one with high statistics entering into the track fit (each track will contain $\mathrm{O}(100)$ strip hits).

The upper right plot in Figure 7.6 demonstrates the powerful filtering effect that the rock has on the hadron activity surrounding the neutrino interaction vertex. Over $90 \%$ of events selected have a reconstructed shower energy below $500 \mathrm{MeV}$. The tail at larger shower energy represents either contained-vertex events (where we can measure the part of the shower that is also contained) or rock vertex events taking place very close to the cavern wall (where a spray of hadrons can accompany the muon and penetrate into the detector). 

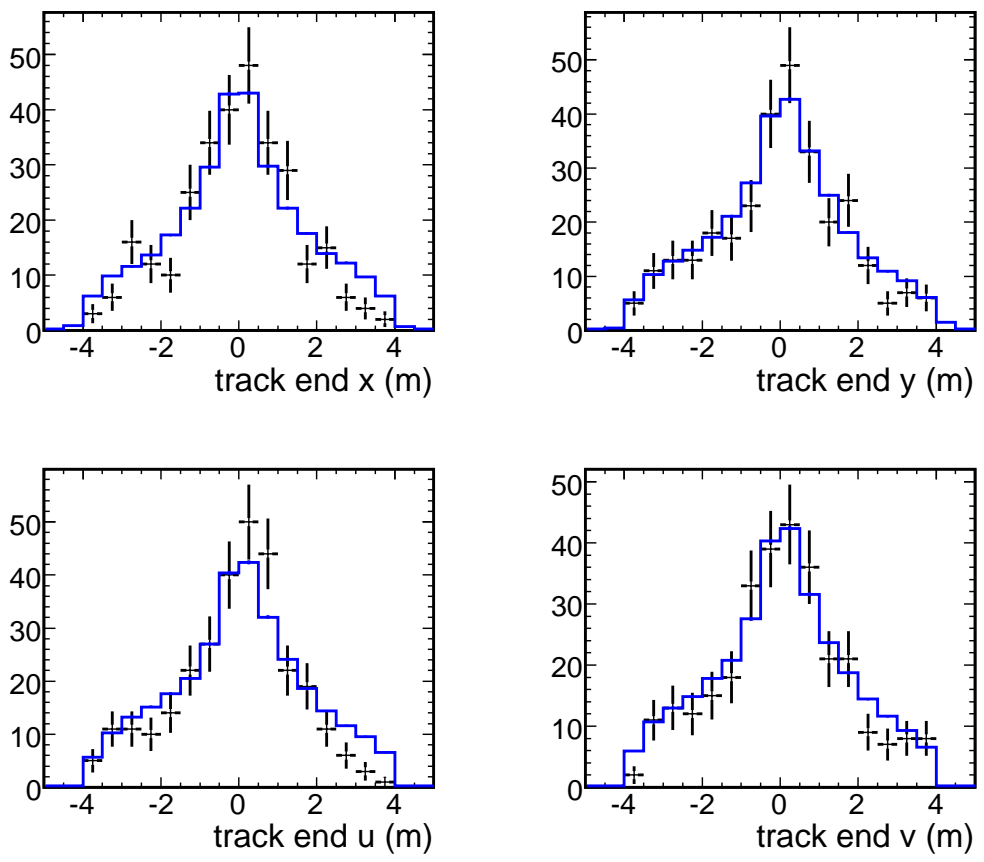

Figure 7.5: Reconstructed track endpoint locations. Top left: track endpoint location in $\mathrm{x}$. Top right: track endpoint location in y. Bottom left: track endpoint location in $\mathrm{u}$. Bottom right: track endpoint location in $\mathrm{v}$.

The lower left plot in Figure 7.6 shows the reconstructed direction cosine $\cos \theta_{\text {beam }}$, which is used along with the reconstructed track momentum in the simultaneous 2-D oscillation fit described in Section 6.1.1. The peak near $\cos \theta_{\text {beam }}=1.0$ represents muons that arrive nearly along the neutrino beam direction. Most such muons enter the front of the detector, while those having lower values of $\cos \theta_{\text {beam }}$ have a steeper angle of attack and usually enter the sides of the detector, where acceptance is larger for such events.

\subsubsection{Track momentum measurement}

The top two plots in Figure 7.7 display the two quantities used to determine track momentum in the Far Detector. The upper left plot shows the distribution of momentum as measured by its range within the detector. Since muon energy loss characteristics are well-known for the detector materials (i.e. straggling is small), a precise determination of 

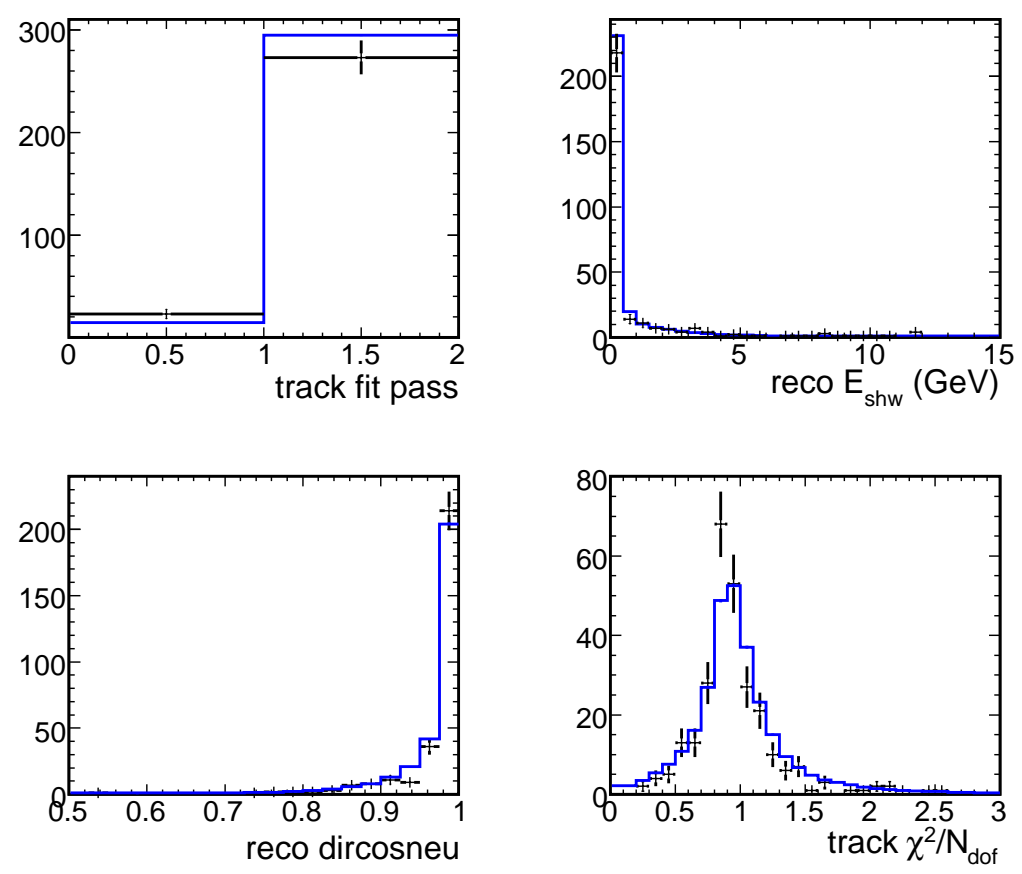

Figure 7.6: Various reconstructed event quantities. Top left: quality of track fit. Top right: reconstructed shower energy. Bottom left: cosine of track vertex direction with respect to beam axis. Bottom right: track fit $\chi^{2} / N_{\text {d.o.f. }}$.

the initial muon momentum can be made if the track has an endpoint that is contained within the detector. In this plot, it is evident that there is an energy-dependent deficit at low-momentum. One can also discern the low detection efficiency for the lowest-energy muons. In the author's Monte Carlo model (see Section 3.2), which recorded the muon energy as it crossed the detector box, there were initially no detection inefficiencies. A continuous distribution resulted at low momentum. Using the author's model, this lower event rate in the first bin was reproduced by discarding all muons below $\sim 200 \mathrm{MeV}$, where the detection and reconstruction efficiency of the MINOS Far Detector drops off. We observe a substantial decrease in the lowest-momentum bin, representing the requirement that a muon penetrates at minimum four planes of steel to generate enough hits to form a reconstructed track. Also evident is a bump at $P_{\text {range }} \sim 20 \mathrm{GeV} / \mathrm{c}$, which represents the highest-energy muons that exit the detector with momentum to spare. 
The upper right plot in Figure 7.7 displays the distribution of the quantity $q / p$, the inverse momentum (curvature) of the reconstructed track, signed by the charge of the muon. Since the selection cuts remove any track reconstructed with a positive charge, only events with $q / p \leq 0$ are shown here. About $15 \%$ of selected events exit the detector and must use a momentum determination of $|1 / q / p|$. Only such events that have a confident curvature measurement are selected for this analysis (see Section 4.2.1). This confidence level is determined by the quantity $\sigma_{q / p}$, assigned by the reconstruction algorithm as a measure of the goodness of the momentum determination.

The lower right plot in Figure 7.7 shows the final reconstructed momentum value as selected for this analysis. In the selected data sample, $86 \%$ of events use $P_{\text {reco }}=P_{\text {range }}$, as their track endpoints fall within the fiducial volume of the detector. The remainder of this distribution is assigned by the curvature momentum determination, derived from the $q / p$ value. A low-momentum deficit is apparent, suggestive of an oscillation signature.

\subsubsection{Particle ID distribution}

Figure 7.8 displays the three input variables to the Particle IDentification (PID) parameter, introduced in Section 4.3. The upper left plot displays the fraction of the total event pulse height contained in the track. As most rock muons are accompanied by little to no shower activity, this distribution peaks towards a value of one. The upper right plot displays the track pulse height per plane, which is related to the muon $\mathrm{dE} / \mathrm{dX}$ characteristic curve. The lower left plot shows the total event length, which is directly related to the track momentum. A similar deficit at low momentum is evident, as well as $\sim 10$ events that enter the front of the detector and exit the rear, having event length equal to the 486 total planes of the Far Detector. The lower right plot displays the resulting distribution of PID values when the three input variables are combined for each event as described in Section 4.3. The distribution is skewed towards more positive values, indicating that the event sample on the whole has more charged-current qualities. The cut to remove neutral-current-like events has been applied for pid0 $<-0.5$ to minimize the effect of the $\mathrm{NC}$ background.

\subsubsection{Two-dimensional momentum-direction histogram}

When the observed data is binned in reconstructed momentum and angle, a comparison can be made with the expected 2-D distribution shown in Figure 5.12. By subtracting 

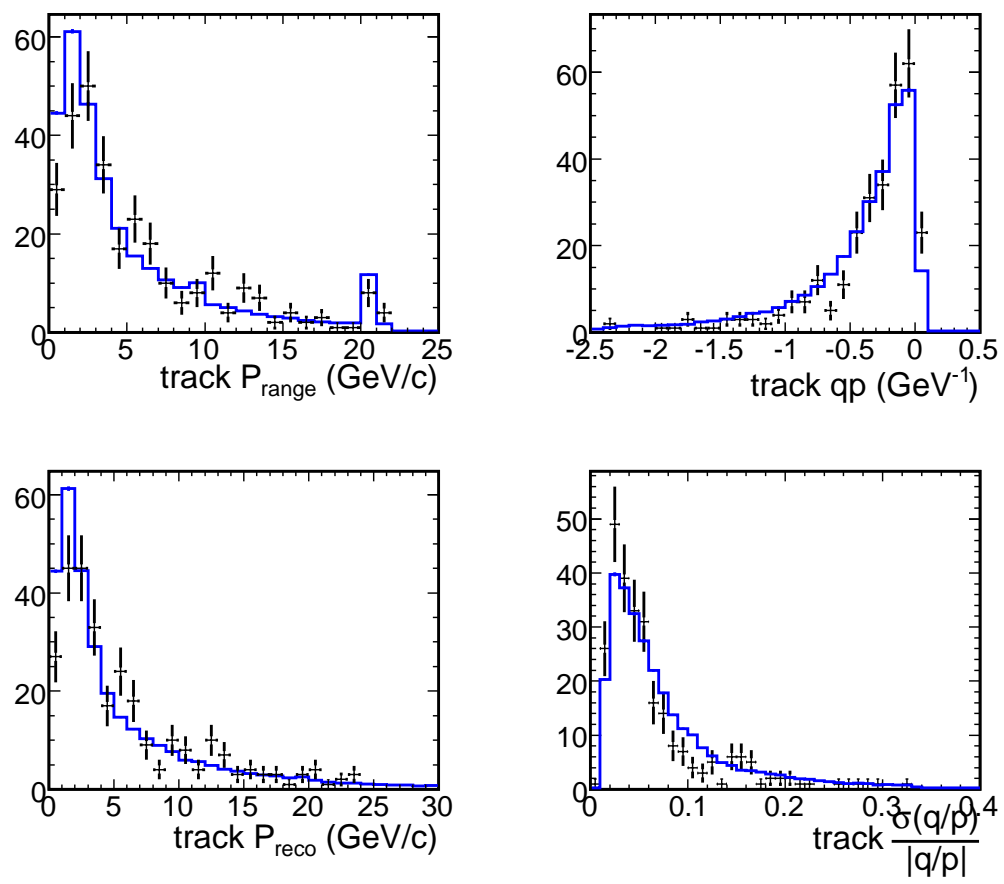

Figure 7.7: Reconstructed track momentum from different measurement techniques. Top left: track momentum from range. Top right: track inverse momentum from curvature. Bottom left: track momentum as selected for analysis. Bottom right: track curvature error divided by curvature.

the expected distribution from the observed data distribution, deficits or surpluses in individual regions of the distribution can be displayed. Figure 7.9 shows this difference. It is clear that the region of low momentum and large direction cosine demonstrates the largest deficit. Also present is an excess in the data over the Monte Carlo at higher momentum and large direction cosine.

The largest deficits are present in the $\cos \theta_{\text {beam }}=0.9-1.0$ and $P_{\text {reco }}<3.0 \mathrm{GeV} / \mathrm{c}$ region. The largest excess is present in the $\cos \theta_{\text {beam }}=1.0$ and $5.0 \mathrm{GeV} / \mathrm{c}<P_{\text {reco }}<6.0 \mathrm{GeV} / \mathrm{c}$ bin. Further excess is observed in the $\cos \theta_{\text {beam }}=1.0$ and $P_{\text {reco }}>10.0 \mathrm{GeV} / \mathrm{c}$ region. 

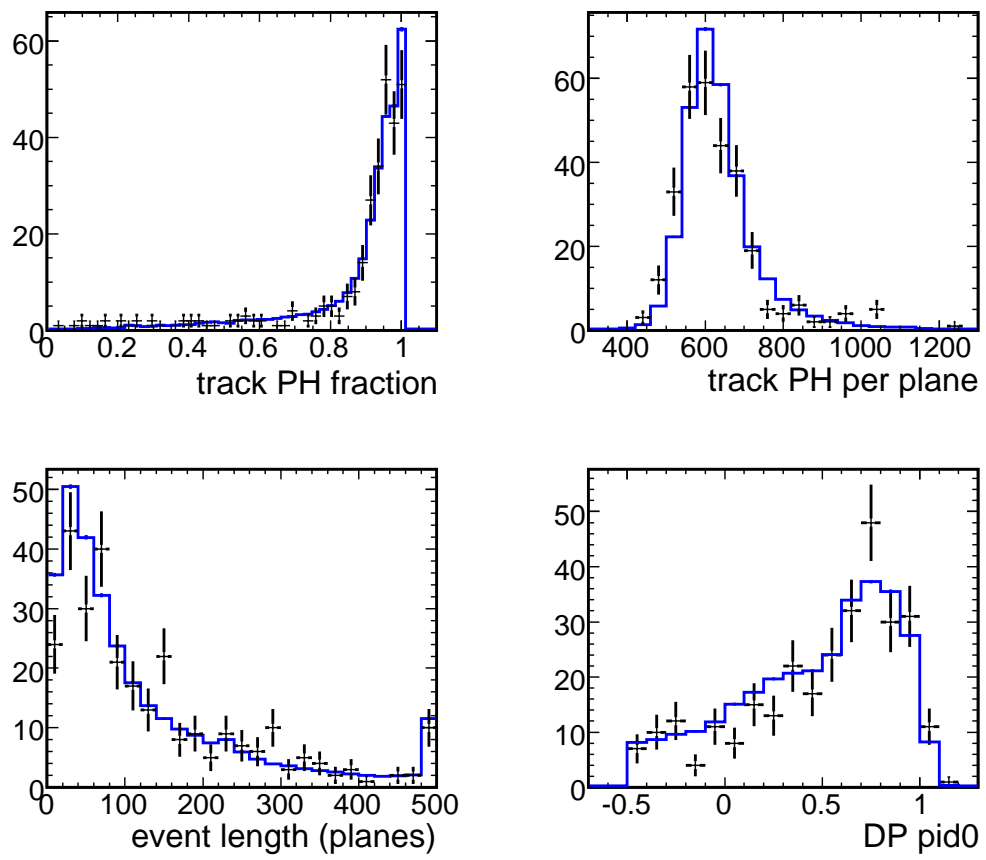

Figure 7.8: Three reconstructed variables that determine event PID and the resulting PID distribution. Top left: fraction of event pulse height contributed by track. Top right: track pulse height per plane. Bottom left: number of planes in event. Bottom right: standard PID variable developed by D. Petyt (cut applied below -0.5 to remove NC-like events).

\subsection{Hypothesis test}

The null hypothesis $H_{0}$ is defined to represent the case in which there are no physical mechanisms for $\nu_{\mu}$ disappearance; in this context, no oscillations can occur. If $H_{0}$ is true, MINOS will observe the expected number of muon events in the antifiducial volume, modulo the statistical fluctuations characteristic of a counting experiment observing a Poisson distribution in each bin of reconstructed momentum and direction.

The alternative hypothesis $H_{1}$ is defined to represent the case in which neutrino oscillation is the physical mechanism for any observed deviation from the expected number of muon events, characterized by the oscillation probability 


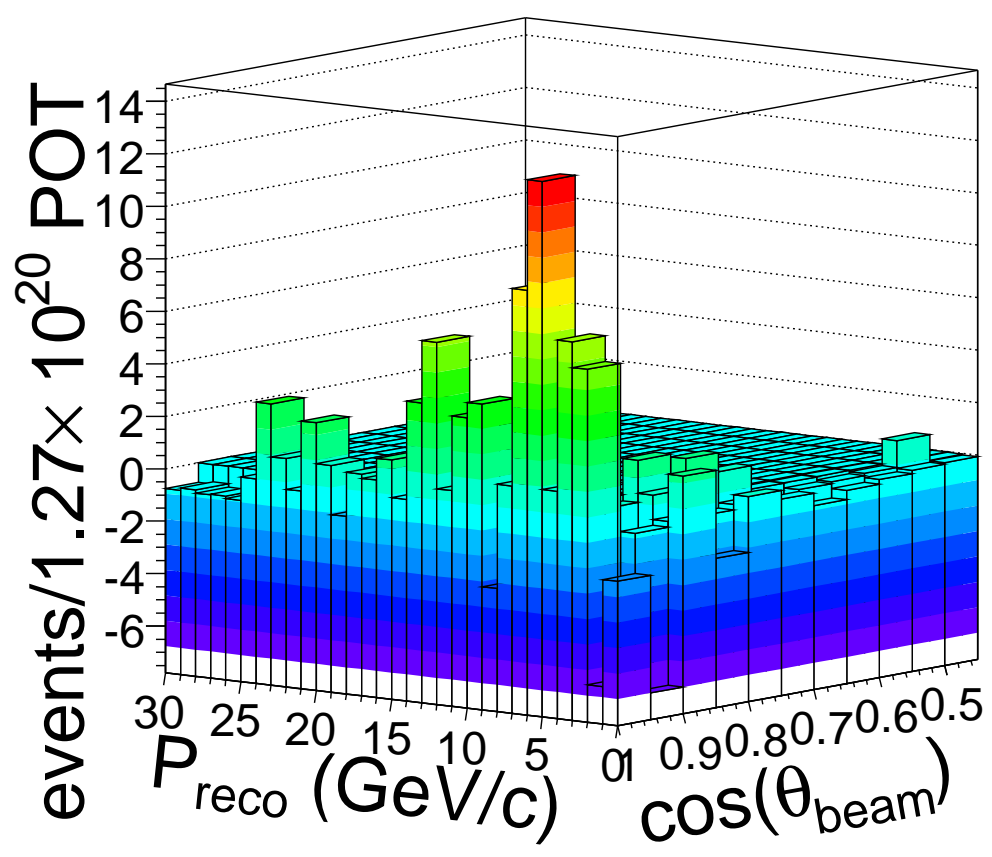

Figure 7.9: Observed minus expected distributions in reconstructed momentum versus angle. An observed deficit presents as a dip below the zero line; an observed excess presents as a peak above zero.

$$
P\left(\nu_{\mu} \rightarrow \nu_{\tau}\right)=\sin ^{2} 2 \theta_{23} \sin ^{2}\left(1.27 \Delta m_{32}^{2} \frac{L}{E}\right),
$$

where $\theta_{23}$ and $\Delta m_{32}^{2}$ are the mixing angle and difference in mass squared in $\mathrm{eV}^{2} / \mathrm{c}^{4}$ between the mass eigenstates $\nu_{3}$ and $\nu_{2}, L$ is the distance traveled in $\mathrm{km}$, and $E$ is the neutrino energy in $\mathrm{GeV}$. If $H_{1}$ is true, MINOS will observe a distribution of muon events that is well-described by a Monte Carlo prediction modified to include $\nu_{\mu} \rightarrow \nu_{\tau}$ oscillations. The goodness-of-fit obtained by finding the fit parameters $\left|\Delta m_{32}^{2}\right|$ and $\sin ^{2} 2 \theta_{23}$ that best describe the observed data is the basic measure of how well $H_{1}$ describes the observation.

The hypothesis test defines a significance level $\alpha$ that represents the probability of rejecting $H_{0}$ if it is true. In some tests, this is referred to as the p-value. Statistical fluctuations can always mimic a signal or deficit, with a well-defined probability to make an observation that is consistent with $H_{0}$. If one generated a large enough ensemble of 
Monte Carlo experiments which do not model any $\nu_{\mu}$ disappearance, some percentage of these experiments would be consistent with the decision to reject $H_{0}$ and accept the best-fit hypothesis $H_{1}$.

It is important to note here that, in the case where the $-2 \mathcal{L}=\chi_{P}^{2}$ approximation (see Appendix C.2) is valid, one would take the actual value of the $\chi^{2}$ fit to the Monte Carlo expectation under $H_{0}$ (no disappearance) as the goodness-of-fit measure and use a lookup table for $\chi^{2}$ distributions to find the corresponding p-value. Due to the many low-count bins present in the fit presented here, the $\chi_{P}^{2}$ approximation breaks down. Therefore, the actual value of $\mathcal{L}_{H_{0}}$ cannot be used directly to determine the goodness-of-fit.

The approach taken here is to consider the difference $\Delta \mathcal{L}=\mathcal{L}_{H_{1}}-\mathcal{L}_{H_{0}}$ in log likelihood between the best-fit oscillation hypothesis and the no-disappearance hypothesis. While the absolute value of the chosen $\chi^{2}$ function in Section 6.1.2 cannot be used here to evaluate goodness-of-fit, the absolute difference $\Delta \mathcal{L}$ provides a means for directly comparing the hypotheses $H_{0}$ and $H_{1}$ (see Appendix C.1). When "opening the box" by performing the analysis on the full data set, it is important to interpret the best-fit oscillation parameters in light of the significance level of the measurement. In the final fit of MINOS Far Detector antifiducial data events, the difference in log likelihood between the best-fit point in oscillation parameter space $H_{1}$ and the no-disappearance condition $H_{0}$ (located at the boundaries at which the oscillation probability is zero, either at $\left|\Delta m_{32}^{2}\right|=0$ or at $\sin ^{2} 2 \theta_{23}=0$ ) is found to be $\Delta \mathcal{L}=5.46011$. Following the prescription in Appendix C.1, the ratio of the likelihoods for the best-fit hypothesis over the null hypothesis is

$$
B=e^{\Delta \mathcal{L}}=23.5123
$$

and the significance level is

$$
\alpha=1-\frac{B}{B+1}=4.23508 \times 10^{-3} .
$$

The null hypothesis $H_{0}$ is rejected at confidence level $1-\alpha=99.5 \%$ and the author concludes that $\nu_{\mu}$ disappearance has been observed in the NuMI beam-induced rock and non-fiducial muon sample in the MINOS Far Detector.

\subsection{Fit results for oscillation parameters}

Having rejected the null (no-disappearance) hypothesis $H_{0}$ at better than $99 \%$ confidence level, the observed data are fit to the alternative (oscillation) hypothesis $H_{1}$. This 
characterizes the probability (see Section B.1) for a muon neutrino created in the NuMI beamline to interact as a muon neutrino at the Soudan Mine as

$$
P\left(\nu_{\mu} \rightarrow \nu_{\mu}\right)=1-\sin ^{2} 2 \theta_{23} \sin ^{2}\left(1.27 \Delta m_{32}^{2} \frac{L}{E}\right) .
$$

Following the prescription laid out in Section 6.1, the log likelihood for observing the data under the oscillation hypothesis was maximized by varying two oscillation parameters $\left|\Delta m_{32}^{2}\right|$ and $\sin ^{2} 2 \theta_{23}$ as well as the penalty terms $N_{\pi}$ and $N_{K}$ (sometimes known as nuisance parameters), multiplicative factors to the pion and kaon templates that account for the uncertainty in the absolute event rates from both the pion and kaon contributions. The best fit to $H_{1}$ is achieved with the parameter values shown in Table 7.1.

\begin{tabular}{|c|c|c|}
\hline \hline Parameter & Best-fit value & $\sigma_{\text {stat }}(68 \%$ C.L. $)$ \\
\hline$\left|\Delta m_{32}^{2}\right|$ & $2.32 \times 10^{-3}$ & $\pm_{0.44}^{0.87} \times 10^{-3}$ \\
$\sin ^{2} 2 \theta_{23}$ & 1.00 & -0.36 \\
$N_{\pi}$ & 1.01 & \pm 0.03 \\
$N_{K}$ & 1.15 & \pm 0.14 \\
\hline \hline
\end{tabular}

Table 7.1: Best-fit values of the oscillation and normalization parameters obtained in final analysis, with statistical uncertainties. Units on $\left|\Delta m_{32}^{2}\right|$ are $\mathrm{eV}^{2} / \mathrm{c}^{4}$.

Statistical uncertainties are reported for the oscillation parameters by fixing the normalization parameters at their best-fit values and varying $\left|\Delta m_{32}^{2}\right|$ and $\sin ^{2} 2 \theta_{23}$ independently and recording the value at which the best-fit $\chi^{2}$ deviates by $\Delta \chi^{2}=1.0$. The observed data (with all cuts applied except for the momentum and angle cuts, applied for the fit) are displayed in Figures 7.10 and 7.11, along with the respective momentum and angle distributions for the unoscillated and best fit Monte Carlo. The best fit does not use these entire distributions; it omits all events above $10 \mathrm{GeV} / \mathrm{c}$. No events were observed below $\cos \theta_{\text {beam }}=0.5$. Statistical uncertainties are reported for each of the normalization parameters by fixing the other three fit parameters and reporting the boundaries at which $\Delta \chi^{2}=1.0$.

It is interesting to note the following: above $10 \mathrm{GeV} / \mathrm{c}$, there is a $14 \%$ excess of events in the data over the Monte Carlo (64 observed, with 56.2 expected). The best fit normalizations were $N_{p i}=1.01$ and $N_{K}=1.15$, performed on only the events below $10 \mathrm{GeV} / \mathrm{c}$. The fact that the kaon contribution (which contributes nearly all events above $10 \mathrm{GeV} / \mathrm{c}$ ) was pulled up by $15 \%$ in this sample below $10 \mathrm{GeV} / \mathrm{c}$ is a strong confirmation that the fit is performing as it should. The fit was in no way informed by the $14 \%$ excess 


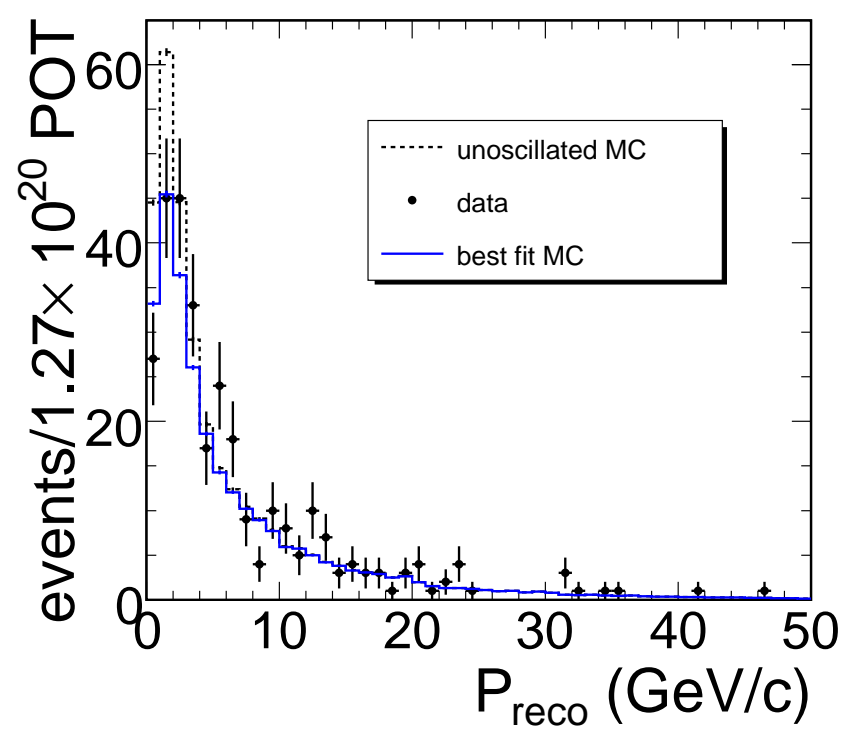

Figure 7.10: Reconstructed track momentum. Data: black points. Unoscillated MC: dotted black lines. Best fit MC: solid blue lines.

above $10 \mathrm{GeV} / \mathrm{c}$, yet it describes it quite well. The advantage of this template fit method is that the muon spectra for the pion and kaon contributions have distinct shapes below $10 \mathrm{GeV} / \mathrm{c}$ (see Figure 6.3).

The single data event below $\cos \theta_{\text {beam }}=0.7$ is the NC-like event discussed in Section 4.2.3 and shown in Figure 4.3. In this region, 1.8 events are expected under the best fit oscillation hypothesis. When the momentum and angle cuts are applied, the resulting distributions are shown in Figures 7.12 and 7.13. These display the 232 data events selected for use in the oscillation fit.

\subsection{Comparison with published MINOS result}

The contours derived from the MINOS fiducial CC analysis are shown alongside the contours from this analysis in Figure 7.14. Due to the degeneracy between the oscillation parameters for the antifiducial analysis, the $\chi^{2}$ surface along the trough of the contours is very shallow. Using an entirely independent sample of Far Detector events, this analysis 


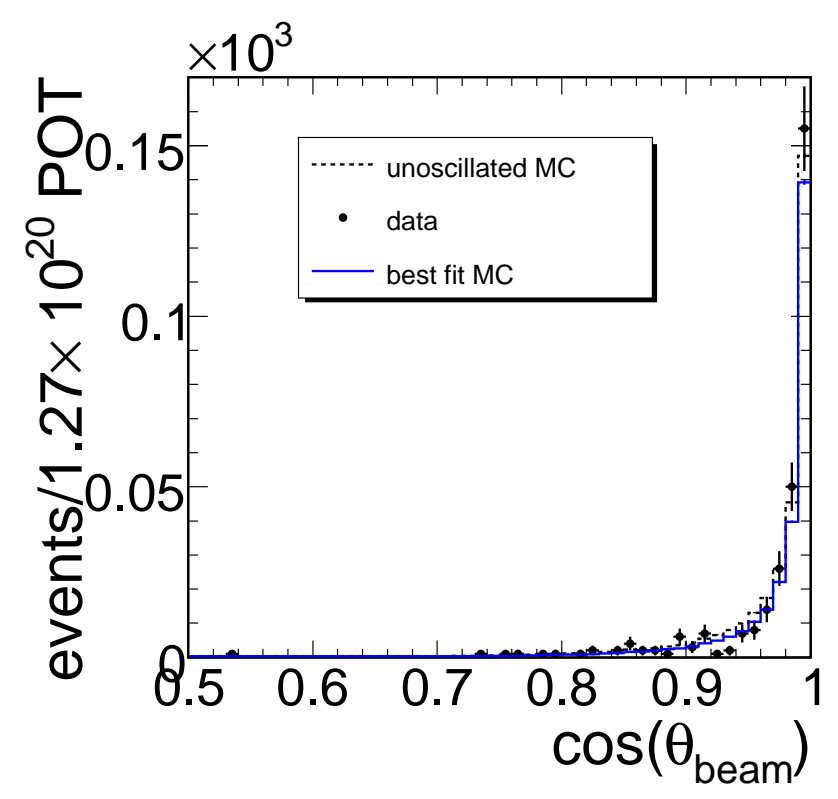

Figure 7.11: Reconstructed track direction. Data: black points. Unoscillated MC: dotted black lines. Best fit MC: solid blue lines.

provides a cross-check on the published fiducial analysis. The antifiducial best fit point lies on the lower boundary of the fiducial $68 \%$ C.L. contour. The $90 \%$ C.L. regions for both analyses are very consistent.

\subsection{Comparison with other published experiments}

The MINOS antifiducial result is shown alongside the results from the K2K and SuperKamiokande experiments in Figure 7.15. The 90\% confidence level contours are displayed for each result. The MINOS antifiducial best-fit point lies in the center of the Super$\mathrm{K}$ region and is consistent with these prior measurements of the atmospheric neutrino oscillation parameters. 


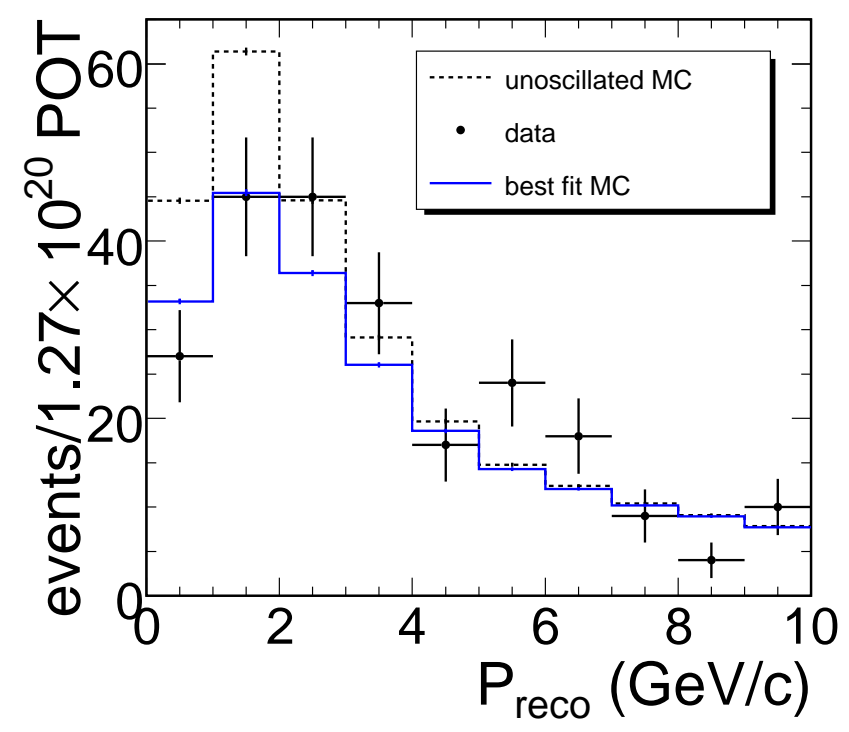

Figure 7.12: Reconstructed track momentum for events used in fit. Data: black points. Unoscillated MC: dotted black lines. Best fit MC: solid blue lines.

\section{7 $\quad$ Future work}

Future work is needed to validate a combined analysis that will use both the fiducial contained vertex neutrino events and the non-fiducial rock and contained vertex events. While the gain in statistical power is desirable, work must be done to reduce the systematic uncertainties of the combined analysis to ensure that they do not overwhelm the decrease in statistical uncertainty on the oscillation parameters. At the $1.27 \times 10^{20} \mathrm{POT}$ exposure, the statistical improvement was estimated to be $8 \%$ on $\left|\Delta m_{32}^{2}\right|$ and $2.5 \%$ on $\sin ^{2} 2 \theta_{23}$. Investigation by D. Petyt also indicates that the ability of MINOS to discriminate against other disappearance models stands to improve significantly by combining the fiducial and antifiducial event samples, due mainly to the different signatures predicted by the models at the high neutrino energies contributing to the rock muon sample.

As of the completion of this document, the Fermilab Tevatron shutdown for the summer of 2007 has begun, concluding the second year of beam on target (Run II). The total accumulated data during NuMI Runs I and II is more than twice the exposure of 


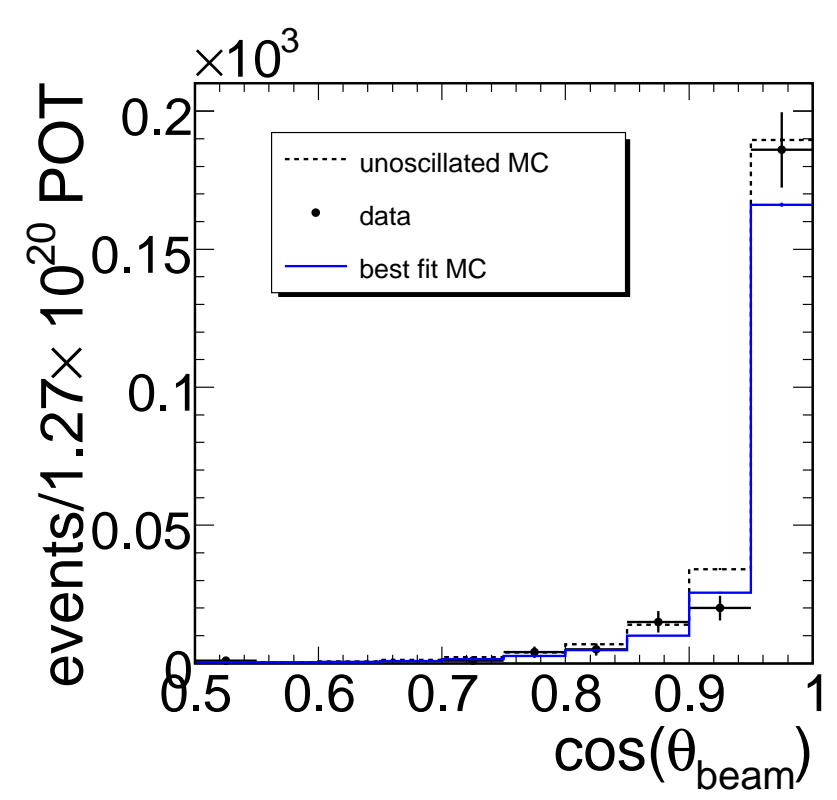

Figure 7.13: Reconstructed track direction for events used in fit. Data: black points. Unoscillated MC: dotted black lines. Best fit MC: solid blue lines.

$1.27 \times 10^{20}$ POT used in this analysis (Run I). Final processing of Run II data is now underway. Figure 7.16 shows the performance of the NuMI beam line during the first two years of operation.

The author will participate in an updated muon analysis that will take advantage of the first two years of beam data in the MINOS detectors. Figure 7.17 shows the expected sensitivity improvement at input oscillation parameters of $\left|\Delta m_{32}^{2}\right|=2.30 \times 10^{-3} \mathrm{eV}^{2} / \mathrm{c}^{4}$ and $\sin ^{2} 2 \theta_{23}=1.0$ for $1.27,3.0$, and $6.0 \times 10^{20}$ protons on target.

\subsection{Conclusions}

During NuMI Run I, with $1.27 \times 10^{20}$ protons accumulated on target, the MINOS Far Detector recorded 232 events below $10 \mathrm{GeV} / \mathrm{c}$ that pass the antifiducial volume selection cuts. In this region, $252.9 \pm 23.7$ events were expected under the assumption of no disappearance. Below $3 \mathrm{GeV} / \mathrm{c}, 117$ events are observed, where $150.2 \pm 16.1$ were expected.

When the observed data below $10 \mathrm{GeV} / \mathrm{c}$ is fit to the oscillation hypothesis, the 


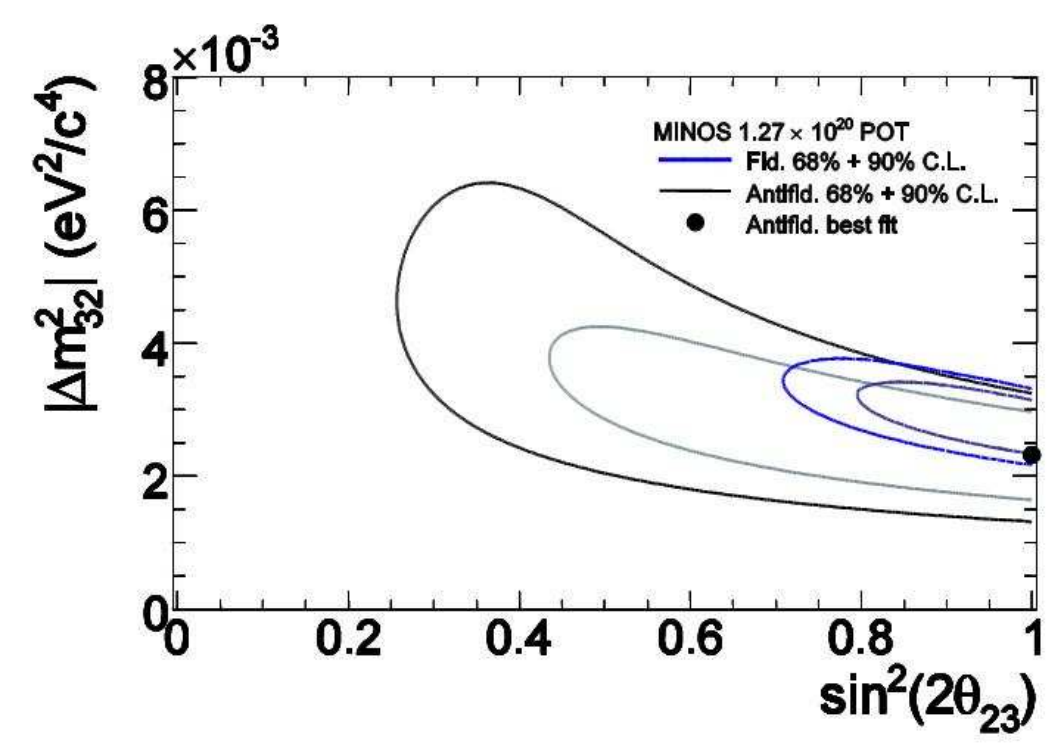

Figure 7.14: Confidence Level contours for the MINOS fiducial and antifiducial analyses.

null hypothesis is ruled out at $99.58 \%$ confidence level. The data are consistent with the oscillation hypothesis, with a best fit occuring at $\left|\Delta m_{32}^{2}\right|=2.32 \times 10^{-3} \mathrm{eV}^{2} / \mathrm{c}^{4}$ and $\sin ^{2} 2 \theta_{23}=1.00$. When only statistical uncertainty is considered, the resulting parameter measurements are

$$
\left|\Delta m_{32}^{2}\right|=2.32 \pm_{0.44}^{0.87} \times 10^{-3} \mathrm{eV}^{2} / \mathrm{c}^{4} \text { and } \sin ^{2} 2 \theta_{23}>0.64 \text { at } 68 \% \text { C.L. }
$$

When systematic uncertainties are included, the results are

$$
\left|\Delta m_{32}^{2}\right|=2.32 \pm_{0.75}^{1.06} \times 10^{-3} \mathrm{eV}^{2} / \mathrm{c}^{4} \text { and } \sin ^{2} 2 \theta_{23}>0.48 \text { at } 68 \% \text { C.L. }
$$

This measurement, using an entirely independent data sample, is consistent with the reported[1] MINOS fiducial volume result from the same $1.27 \times 10^{20}$ POT exposure. 


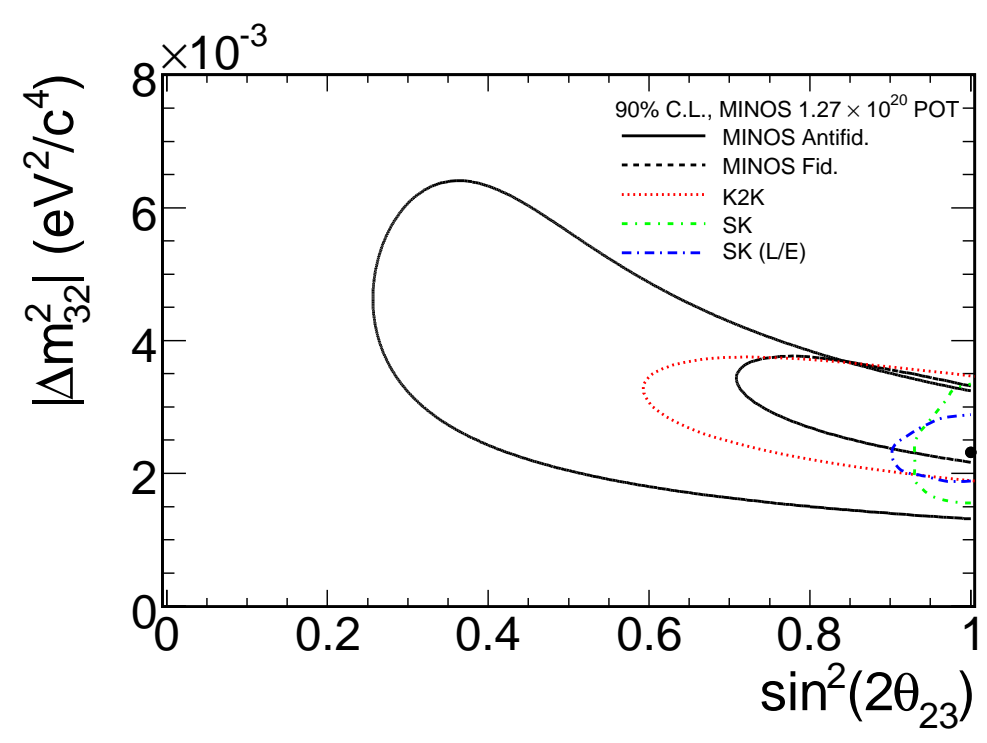

Figure 7.15: Confidence Level contours for the MINOS fiducial and antifiducial analyses, Super-Kamiokande, and K2K experiments.

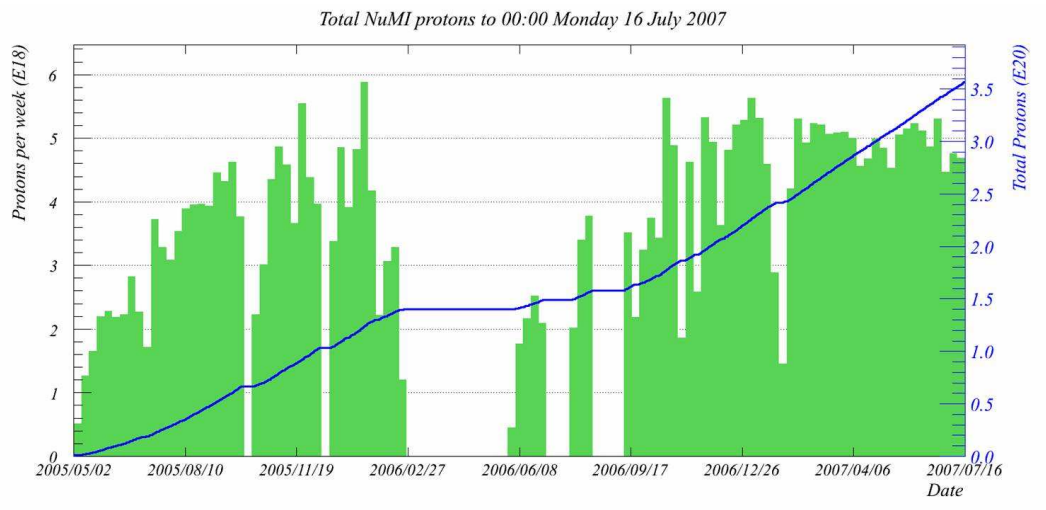

Figure 7.16: Total protons on target (POT) delivered to the NuMI target during the first two years of operation. The gap represents the 2006 Tevatron shutdown. The total exposure in the standard LE-10 running configuration is $\sim 3.0 \times 10^{20}$ POT. 


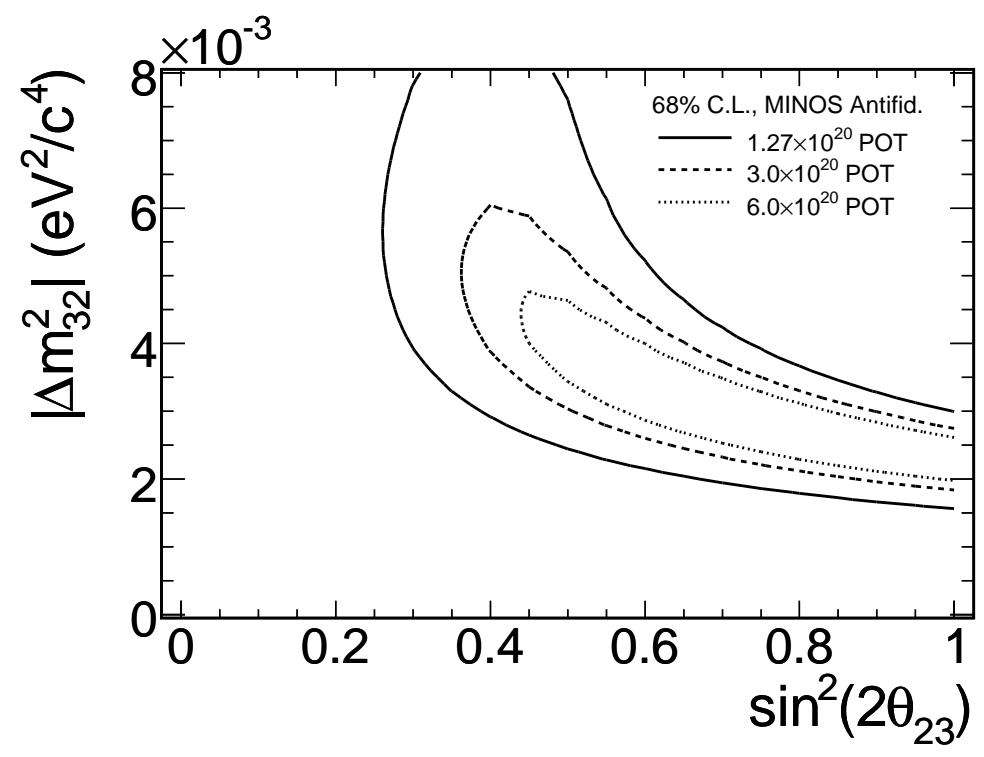

Figure 7.17: Monte Carlo sensitivity 68\% CL contours for the MINOS antifiducial analysis, with two approximate doublings of the Run I data presented here. 


\title{
Appendix A
}

\section{MINOS Collaboration}

\author{
Main Injector Neutrino Oscillation Search
}

June 2007

P. Adamson ${ }^{8,12}$, C. Andreopoulos ${ }^{17}$, K.E. Arms ${ }^{13}$, R. Armstrong ${ }^{11}$, D.J. Auty ${ }^{20}$, D.S. Ayres ${ }^{1}$, B. Baller ${ }^{8}$, B. Barish ${ }^{5}$, G. Barr ${ }^{15}$, R. H. Bernstein ${ }^{8}$, D. Bhattacharya ${ }^{16}$, M. Bishai ${ }^{4}$, A. Blake ${ }^{6}$, B. Bock ${ }^{14}$, G.J. Bock ${ }^{8}$, J. Boehm $^{9}$, D.J. Boehnlein ${ }^{8}$, D. Bogert ${ }^{8}$, C. Bower ${ }^{11}$, E. Buckley-Geer ${ }^{8}$, S. Cavanaugh ${ }^{9}$, J.D. Chapman ${ }^{6}$, D. Cherdack ${ }^{23}$, S. Childress ${ }^{8}$, J.H. Cobb ${ }^{15}$, S.J. Coleman ${ }^{26}$, A.J. Culling ${ }^{6}$, J.K. de Jong ${ }^{10}$, M. Dierckxsens ${ }^{4}$, M.V. Diwan ${ }^{4}$, M. Dorman ${ }^{12,17}$, D. Drakoulakos ${ }^{2}$, S.A. Dytman ${ }^{16}$, A.R. Erwin ${ }^{27}$, C.O. Escobar ${ }^{24}$, J.J. Evans ${ }^{15}$, E. Falk Harris ${ }^{20}$, G.J. Feldman ${ }^{9}$, R. Ford ${ }^{8}$, M.V. Frohne ${ }^{3}$, H.R. Gallagher ${ }^{23}$, A. Godley ${ }^{18}$, J. Gogos ${ }^{13}$, M.C. Goodman ${ }^{1}$, P. Gouffon ${ }^{25}$, R. Gran ${ }^{14}$, E.W. Grashorn ${ }^{13}$, N. Grossman ${ }^{8}$, K. Grzelak ${ }^{15}$, A. Habig ${ }^{14}$, D. Harris ${ }^{8}$, P.G. Harris ${ }^{20}$, J. Hartnell ${ }^{17}$, R. Hatcher ${ }^{8}$, K. Heller ${ }^{13}$, A. Himmel ${ }^{5}$, A. Holin ${ }^{12}$, C. Howcroft ${ }^{5}$, J. Hylen ${ }^{8}$, G.M. Irwin ${ }^{19}$, M. Ishitsuka ${ }^{11}$, D.E. Jaffe ${ }^{4}$, C. James ${ }^{8}$, D. Jensen ${ }^{8}$, T. Kafka ${ }^{23}$, S.M.S. Kasahara ${ }^{13}$, M.S. Kim ${ }^{16}$, G. Koizumi ${ }^{8}$, S. Kopp ${ }^{22}$, M. Kordosky ${ }^{12}$, D.J. Koskinen ${ }^{12}$, A. Kreymer ${ }^{8}$, S. Kumaratunga ${ }^{13}$, K. Lang ${ }^{22}$, J. Ling ${ }^{18}$, P.J. Litchfield ${ }^{13}$, R.P. Litchfield ${ }^{15}$, P. Lucas ${ }^{8}$, W.A. Mann ${ }^{23}$, A. Marchionni ${ }^{8}$, M.L. Marshak ${ }^{13}$, J.S. Marshall ${ }^{6}$, N. Mayer ${ }^{14}$, A.M. McGowan ${ }^{1,13}$, J.R. Meier ${ }^{13}$, M.D. Messier ${ }^{11}$, C.J. Metelko ${ }^{17}$, W.H. Miller ${ }^{13}$, S.R. Mishra ${ }^{18}$, A. Mislivec ${ }^{14}$, C.D. Moore ${ }^{8}$, J. Morfín ${ }^{8}$, L. Mualem ${ }^{5}$, S. Mufson ${ }^{11}$, S. Murgia ${ }^{19}$, J. Musser ${ }^{11}$, D. Naples ${ }^{16}$, J.K. Nelson ${ }^{26}$, H. Newman ${ }^{5}$, R.J. Nichol ${ }^{12}$, T.C. Nicholls ${ }^{17}$, J.P. Ochoa-Ricoux ${ }^{5}$, W.P. Oliver ${ }^{23}$, T. Osiecki ${ }^{22}$, R. Ospanov ${ }^{22}$, J. Paley ${ }^{11}$, V. Paolone ${ }^{16}$, A. Para ${ }^{8}$, T. Patzak ${ }^{7}$, Ž. Pavlović ${ }^{22}$, G. Pawloski ${ }^{19}$, G.F. Pearce ${ }^{17}$, 
C.W. Peck ${ }^{5}$, E.A. Peterson ${ }^{13}$, D.A. Petyt ${ }^{13}$, R. Pittam ${ }^{15}$, R.K Plunkett ${ }^{8 \dagger}$, R.A. Rameika ${ }^{8}$, T.M. Raufer ${ }^{15}$, B. Rebel $^{8}$, J. Reichenbacher ${ }^{1}$, D.E. Reyna ${ }^{1}$, P.A. Rodrigues ${ }^{15}$, C. Rosenfeld ${ }^{18}$, H.A. Rubin ${ }^{10}$, K. Ruddick ${ }^{13}$, M.C. Sanchez ${ }^{9}$, N. Saoulidou ${ }^{8}$, J. Schneps ${ }^{23}$, P. Schreiner ${ }^{3}$, P. Shanahan ${ }^{8}$, A. Sousa ${ }^{15}$, B. Speakman ${ }^{13}$,

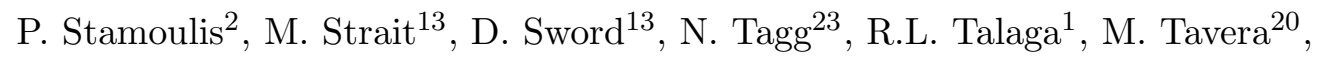
E. Tetteh-Lartey ${ }^{21}$, J. Thomas ${ }^{12}$, M.A. Thomson ${ }^{6}$, G. Tinti ${ }^{15}$, G. Tzanakos ${ }^{2}$, J. Urheim ${ }^{11}$, P. Vahle ${ }^{12}$, B. Viren ${ }^{4}$, D.R. Ward ${ }^{6}$, M. Watabe ${ }^{21}$, A. Weber ${ }^{15}$, R.C. Webb ${ }^{21}$, A. Wehmann ${ }^{8}$, N. West ${ }^{15}$, C. White ${ }^{10}$, S.G. Wojcicki ${ }^{19 \dagger}$, Q.K. Wu ${ }^{18}$, T. Yang ${ }^{19}$, K. Zhang ${ }^{4}$, M. Zois ${ }^{2}$, and R. Zwaska ${ }^{8}$.

\section{$\dagger$ Co-Spokespersons}

Argonne $^{1}-$ Athens $^{2}-$ Benedictine $^{3}-$ Brookhaven $^{4}-$ Caltech $^{5}-$ Cambridge $^{6}$ Collège de France ${ }^{7}$ - Fermilab ${ }^{8}-$ Harvard $^{9}$ - IIT ${ }^{10}$ - Indiana ${ }^{11}$ - UCL-London ${ }^{12}$ Minnesota $^{13}$ - Minnesota-Duluth ${ }^{14}$ - Oxford ${ }^{15}$ - Pittsburgh ${ }^{16}$ - Rutherford $^{17}$ South Carolina ${ }^{18}$ - Stanford ${ }^{19}$ - Sussex $^{20}$ - Texas A\&M ${ }^{21}$ - Texas-Austin ${ }^{22}$ Tufts $^{23}$ - UNICAMP-Brazil ${ }^{24}$ - USP-Brazill ${ }^{25}$ - William \& Mary ${ }^{26}$ - Wisconsin ${ }^{27}$ 


\section{Appendix B}

\section{Physics derivations}

\section{B.1 Survival probability}

In the two-flavor oscillation model, the survival probability for a neutrino created in the $\nu_{\mu}$ flavor eigenstate can be determined in the following way. We begin by assuming that neutrinos can only propagate as mass eigenstates $\nu_{2}$ and $\nu_{3}$, while they are only created as weak flavor eigenstates $\nu_{\mu}$ and $\nu_{\tau}$. We can express the flavor eigenstates as linear superpositions of the mass eigenstates,

$$
\left|\nu_{\mu}(t)\right\rangle=\left|\nu_{2}(t)\right\rangle \cos \theta_{23}+\left|\nu_{3}(t)\right\rangle \sin \theta_{23}
$$

and

$$
\left|\nu_{\tau}(t)\right\rangle=-\left|\nu_{2}(t)\right\rangle \sin \theta_{23}+\left|\nu_{3}(t)\right\rangle \cos \theta_{23}
$$

where $\theta_{23}$ is the mixing angle between $\nu_{2}$ and $\nu_{3}$ and $t$ is the time since creation of the flavor eigenstate in a weak interaction. At $t=0$, we consider a $\nu_{\mu}$ created in the decay of a charged pion or kaon in the NuMI beam line, such that $\nu_{\mu}(0)=1$ and $\nu_{\tau}(0)=0$. Inverting Equations B.1 and B.2, we have

$$
\left|\nu_{2}(t)\right\rangle=\left|\nu_{\mu}(t)\right\rangle \cos \theta_{23}-\left|\nu_{\tau}(t)\right\rangle \sin \theta_{23}
$$

and

$$
\left|\nu_{3}(t)\right\rangle=\left|\nu_{\mu}(t)\right\rangle \sin \theta_{23}+\left|\nu_{\tau}(t)\right\rangle \cos \theta_{23} .
$$

Applying the initial condition at creation $t=0$, we have

$$
\left|\nu_{2}(0)\right\rangle=\left|\nu_{\mu}(0)\right\rangle \cos \theta_{23}
$$


and

$$
\left|\nu_{3}(0)\right\rangle=\left|\nu_{\mu}(0)\right\rangle \sin \theta_{23} .
$$

We can express the time evolution of the mass eigenstates (assuming infinite plane waves) as

$$
\left|\nu_{2}(t)\right\rangle=\left|\nu_{2}(0)\right\rangle e^{-i E_{2} t}
$$

and

$$
\left|\nu_{3}(t)\right\rangle=\left|\nu_{3}(0)\right\rangle e^{-i E_{3} t}
$$

Inserting into Equation B.1, we find

$$
\left|\nu_{\mu}(t)\right\rangle=\left|\nu_{2}(0)\right\rangle e^{-i E_{2} t} \cos \theta_{23}+\left|\nu_{3}(0)\right\rangle e^{-i E_{3} t} \sin \theta_{23} .
$$

Using the initial conditions in Equations B.5 and B.6, this becomes

$$
\left|\nu_{\mu}(t)\right\rangle=\left|\nu_{\mu}(0)\right\rangle e^{-i E_{2} t} \cos ^{2} \theta_{23}+\left|\nu_{\mu}(0)\right\rangle e^{-i E_{3} t} \sin ^{2} \theta_{23} .
$$

We define the survival amplitude to find $\nu_{\mu}$ at time $t$ by

$$
A_{\mu}(t)=\left\langle\nu_{\mu}(0) \mid \nu_{\mu}(t)\right\rangle=e^{-i E_{2} t} \cos ^{2} \theta_{23}+e^{-i E_{3} t} \sin ^{2} \theta_{23}
$$

and the survival probability by

$$
\begin{aligned}
P_{\mu}(t) & =A_{\mu}(t) A_{\mu}(t)^{*}=\left(e^{-i E_{2} t} \cos ^{2} \theta_{23}+e^{-i E_{3} t} \sin ^{2} \theta_{23}\right)\left(e^{+i E_{2} t} \cos ^{2} \theta_{23}+e^{+i E_{3} t} \sin ^{2} \theta_{23}\right) \\
& =\cos ^{4} \theta_{23}+\sin ^{4} \theta_{23}+e^{i\left(E_{3}-E_{2}\right) t} \cos ^{2} \theta_{23} \sin ^{2} \theta_{23}+e^{i\left(E_{2}-E_{3}\right) t} \cos ^{2} \theta_{23} \sin ^{2} \theta_{23} \\
& =\cos ^{4} \theta_{23}+\sin ^{4} \theta_{23}+2 \cos \left(\left(E_{3}-E_{2}\right) t\right) \cos ^{2} \theta_{23} \sin ^{2} \theta_{23} .
\end{aligned}
$$

Expressing 1 in a different way, we find

$$
\begin{aligned}
1^{2} & =\left(\cos ^{2} \theta_{23}+\sin ^{2} \theta_{23}\right)^{2}=\cos ^{4} \theta_{23}+\sin ^{4} \theta_{23}+2 \cos ^{2} \theta_{23} \sin ^{2} \theta_{23} \\
& \rightarrow \cos ^{4} \theta_{23}+\sin ^{4} \theta_{23}=1-2 \cos ^{2} \theta_{23} \sin ^{2} \theta_{23} .
\end{aligned}
$$

Inserting this into Equation B.12, the survival probability becomes

$$
\begin{aligned}
P_{\mu}(t) & =1-\left(2 \cos ^{2} \theta_{23} \sin ^{2} \theta_{23}\right)\left[1-\cos \left(\left(E_{3}-E_{2}\right) t\right)\right] \\
& =1-\left(\frac{1}{2} \sin ^{2} 2 \theta_{23}\right)\left[1-1+2 \sin ^{2}\left(\frac{\left(E_{3}-E_{2}\right) t}{2}\right)\right] \\
& =1-\sin ^{2} 2 \theta_{23} \sin ^{2}\left(\frac{\left(E_{3}-E_{2}\right) t}{2}\right) .
\end{aligned}
$$


Since the original $\nu_{\mu}$ was created as an admixture of $\nu_{2}$ and $\nu_{3}$, these propagating mass eigenstates must necessarily have the same momentum $p=p_{2}=p_{3}$. Since $E^{2}=p^{2}+m^{2}$, we can write $E=\left(p^{2}+m^{2}\right)^{1 / 2}=p\left(1+m^{2} / p^{2}\right)^{1 / 2}$. In the relativistic limit $m^{2}<<p^{2}$ (valid for neutrinos in the NuMI beam line), $E=p+m^{2} / 2 p$. Now,

$$
E_{3}-E_{2}=\frac{m_{3}^{2}-m_{2}^{2}}{2 p}=\frac{c \Delta m_{32}^{2}}{2 E}
$$

where $p=E / c$ for relativistic neutrinos. Restoring all factors of $c$ and $\hbar$, and using the baseline distance traveled by the neutrino $L=c t$, the unitless argument in Equation B.14 becomes

$$
\frac{\left(E_{3}-E_{2}\right) t}{2 \hbar}=\frac{\Delta m_{32}^{2} c^{4}}{2 E} \frac{L}{2 c \hbar}=\frac{\Delta m_{32}^{2} L c^{3}}{4 E \hbar}=\frac{\Delta m_{32}^{2} L}{E} \frac{1}{4 \hbar c}
$$

where the units in the last step are chosen such that a factor $c^{-4}$ is introduced: $\Delta m_{32}^{2}$ is in $\mathrm{eV}^{2}, L$ is in $\mathrm{km}$, and $E$ is in $\mathrm{GeV}$. The quantity $\hbar c=197.326968 \mathrm{MeV}$ fm can be introduced (with appropriate unit conversion) to find

$$
\frac{1}{4 \hbar c}=1.26693276 \mathrm{GeV}^{-1} \mathrm{~km}^{-1} .
$$

Finally, we arrive at the survival probability for a created $\nu_{\mu}$ of energy $E$ to interact as a $\nu_{\mu}$ after traveling a distance $L$ :

$$
P\left(\nu_{\mu} \rightarrow \nu_{\mu}\right)=1-\sin ^{2} 2 \theta_{23} \sin ^{2}\left(\frac{1.267 \Delta m_{32}^{2} L}{E}\right)
$$

\section{B.2 Energy/angle}

In the NuMI beam line, both $\pi^{+}$and $K^{+}$are created in different proportions, along with $\pi^{-}$and $K^{-}$that give rise to $\bar{\nu}_{\mu}$ (excluded from this analysis). The $\nu_{\mu}$ spectrum up to $E \sim 30 \mathrm{GeV}$ resulting from the decay of these charged hadrons is dominated by pions, while the spectrum above this energy is dominated by kaons. The kinematics of the two-body decays $\pi \rightarrow \mu+\nu_{\mu}$ and $K \rightarrow \mu+\nu_{\mu}$ demonstrate why the spectrum is divided in this way.

Rest frame quantities are denoted with an asterisk and lab frame quantities without one. The derivation is generalized for the parent hadron $h=\pi$ or $K$. The rest frame is related to the lab frame by a Lorentz boost, characterized by $\beta=v / c$ and $\gamma=$ $\left(1-\beta^{2}\right)^{-1 / 2}$. The three-vector momentum components are denoted without boldface 
$(p, E)$ and four-momentum with $(\mathbf{p})$. The angle between the initial hadron momentum and the final neutrino momentum is labeled as $\theta$. The longitudinal momentum of the neutrino is labeled $p_{\|}$and the transverse momentum $p_{\perp}$. The neutrino energy is labeled $E_{\nu}$. Natural units are used, where $c=1$.

The momentum-energy four-vector components transform in the same manner as the position-time four-vector, relating the rest-frame to the lab frame:

$$
p_{\|}=\gamma\left(p_{\|}^{*}-\beta E_{\nu}^{*}\right)
$$

and

$$
E_{\nu}=\gamma\left(E_{\nu}^{*}-\beta p_{\|}^{*}\right)
$$

Neglecting the mass of the neutrino (i.e. setting $E_{\nu}=p$ ), the energy and momentum are related through

$$
p_{\|}^{*}=E_{\nu}^{*} \cos \theta^{*} .
$$

Inserting this into Equations B.19 and B.20 yields

$$
p_{\|}=\gamma E_{\nu}^{*}\left(\cos \theta^{*}-\beta\right)
$$

and

$$
E_{\nu}=\gamma E_{\nu}^{*}\left(1-\beta \cos \theta^{*}\right)
$$

Four-momentum must be conserved in the decay:

$$
\mathbf{p}_{\mathbf{h}}=\mathbf{p}_{\mu}+\mathbf{p}_{\nu} \text { or } \mathbf{p}_{\mu}=\mathbf{p}_{\mathbf{h}}-\mathbf{p}_{\nu}
$$

Squaring both sides leads to

$$
\mathbf{p}_{\mu}{ }^{2}=\mathbf{p}_{\mathbf{h}}{ }^{2}+\mathbf{p}_{\nu}{ }^{2}-2 \mathbf{p}_{\mathbf{h}} \cdot \mathbf{p}_{\nu}
$$

Since $\mathbf{p}^{2}=E_{\nu}^{2}-\mathrm{p}^{2}=\mathrm{m}^{2}$, Equation B.25 becomes

$$
m_{\mu}^{2}=m_{h}^{2}+m_{\nu}^{2}-2 \mathbf{p}_{\mathbf{h}} \cdot \mathbf{p}_{\nu} .
$$

Since the scalar product of four-momenta is Lorentz invariant, it can be evaluated in the hadron rest frame, where $\mathbf{p}_{\mathbf{h}}=\left(0,0,0, m_{h}\right)$ and $\mathbf{p}_{\nu}=\left(0, p_{\perp}, p_{\|}, E_{\nu}^{*}\right)$. Ignoring the neutrino mass, this leads to

$$
m_{\mu}^{2}-m_{h}^{2}=-2 m_{h} E_{\nu}^{*}
$$


The transverse momentum of the neutrino is invariant under this boost:

$$
p_{\perp}^{*}=p_{\perp}=E_{\nu}^{*} \sin \theta^{*}=E_{\nu} \sin \theta .
$$

Combining Equations B.28 and B.22,

$$
\tan \theta=\frac{p_{\perp}}{p_{\|}}=\frac{\sin \theta^{*}}{\gamma\left(\cos \theta^{*}-\beta\right)}
$$

To simplify the ensuing algebra, define $a=\tan \theta$ and $b=\cos \theta^{*}$. Rearranging Equation B.29 leads to:

$$
\gamma a=\frac{\sin \theta^{*}}{b-\beta} \rightarrow \gamma^{2} a^{2}=\frac{\sin ^{2} \theta^{*}}{(b-\beta)^{2}} .
$$

In the relativistic regime encountered in the NuMI beam line (with the parent hadrons resulting from the collision of $120 \mathrm{GeV}$ protons on the carbon target nuclei) $\beta \rightarrow 1$. Carrying out some simplification,

$$
\begin{gathered}
\gamma^{2} a^{2}=\frac{\sin ^{2} \theta^{*}}{(b-\beta)^{2}}=\frac{1-\cos ^{2} \theta^{*}}{(1-b)^{2}}=\frac{(1+b)(1-b)}{(1-b)^{2}}=\frac{1+b}{1-b}, \\
\gamma^{2} a^{2}-\gamma^{2} a^{2} b=1+b \rightarrow b=\frac{\gamma^{2} a^{2}-1}{1+\gamma^{2} a^{2}},
\end{gathered}
$$

and

$$
1-b=\frac{1+\gamma^{2} a^{2}-\gamma^{2} a^{2}+1}{1+\gamma^{2} a^{2}}=\frac{2}{1+\gamma^{2} a^{2}} .
$$

Inserting this into Equation B.23,

$$
E_{\nu}=\gamma E_{\nu}^{*} \frac{2}{1+\gamma^{2} a^{2}}
$$

Using $\gamma=E_{h} / m_{h}$, restoring the $a$ term, and combining with Equation B.27 leads to:

$$
E_{\nu}=\gamma \frac{m_{h}^{2}-m_{\mu}^{2}}{2 m_{h}} \frac{2}{1+\gamma^{2} a^{2}}=\frac{E_{h}}{1+\gamma^{2} \tan ^{2} \theta} \frac{m_{h}^{2}-m_{\mu}^{2}}{m_{h}^{2}} .
$$

Finally, in the lab frame $\theta$ will be small given the boost, so one can use $\tan ^{2} \theta=\theta^{2}$ to arrive at:

$$
E_{\nu}=\frac{E_{h}\left(1-m_{\mu}^{2} / m_{h}^{2}\right)}{1+\gamma^{2} \theta^{2}} .
$$

One can understand the crossover and dominance of the kaon contribution at high neutrino energies by evaluating the term in parentheses in the numerator of Equation B.36 for both $h=\pi$ and $K$, using $m_{\mu}=105.7 \mathrm{MeV}$. For charged pions $\left(m_{\pi}=139.6 \mathrm{MeV}\right)$ 
the term evaluates to 0.4269. For charged kaons $\left(m_{K}=493.7 \mathrm{MeV}\right)$ the term is 0.9542 . Given pions and kaons with the same energy $E_{h}$ before decay, the neutrinos parented by kaons will have, on average, approximately twice the energy of those parented by pions.

The $K / \pi$ ratio produced in the NuMI target determines the crossover point where the $\nu_{\mu}$ from the kaon contribution starts to dominate the neutrino spectrum. As modeled in GNuMI, this crossover point occurs near $30 \mathrm{GeV}$. 


\section{Appendix $\mathrm{C}$}

\section{Statistical methods}

\section{C.1 Poisson log likelihood}

One drawback of using a direct $\chi^{2}$ method for the fit performed in this work is the need to have sufficient statistics in each bin of reconstructed momentum and direction. With most events clustered at low momentum and high $\cos \theta_{\text {beam }}$, many bins in the fit have a mere 0-2 observed events. Given these low statistics, the maximum likelihood was chosen as the fit method. The basis for the likelihood used was the Poisson probability for observing $N_{o b s}$ events in a bin with $N_{e x p}$ events:

$$
\mathcal{P}\left(N_{o b s} \mid N_{e x p}\right)=\frac{e^{-N_{e x p}} N_{e x p}^{N_{o b s}}}{N_{o b s} !} .
$$

This probability is normalized to one (by the series definition of the exponential function) when all possible $N_{o b s}$ are summed from zero to infinity, given any $N_{\text {exp }}$ :

$$
\sum_{N_{\text {obs }}=0}^{\infty} \mathcal{P}\left(N_{\text {obs }} \mid N_{\text {exp }}\right)=e^{-N_{\text {exp }}} \sum_{N_{\text {obs }}=0}^{\infty} \frac{N_{\text {exp }}^{N_{\text {obs }}}}{N_{\text {obs }} !}=e^{-N_{\text {exp }}} e^{N_{\text {exp }}}=e^{0}=1 .
$$

Since the count in each bin is an independent measurement, the overall Poisson probability is given by the product of the individual bin probabilities. The natural logarithm of this product is chosen as the likelihood function, since the sum of the logs is easier to work with than the product of the probabilities. Define

$$
\begin{aligned}
\mathcal{L}^{\prime \prime} & =\sum_{\text {all bins }} \ln \mathcal{P}_{\text {bin }}\left(N_{\text {obs }} \mid N_{\text {exp }}\right) \\
& =\sum_{\text {all bins }}-N_{\text {exp }}+N_{o b s} \ln N_{\text {exp }}-\ln N_{o b s} !
\end{aligned}
$$


as the likelihood function. Since one is concerned with differences in $\mathcal{L}^{\prime \prime}$ as a function of the oscillation parameters, the factorial term can be dropped, since it is an observed constant:

$$
\mathcal{L}^{\prime}=\sum_{\text {all bins }}-N_{\text {exp }}+N_{\text {obs }} \ln N_{\text {exp }}
$$

Since this quantity scales with the size of the observed and expected events, it is useful to work with a normalized likelihood function which allows comparison between, say, different POT exposures. By taking the ratio of probabilities, or the difference of logarithms, between the actual case and the case where one expects exactly the number of observed events, one obtains the final normalized likelihood

$$
\begin{aligned}
\mathcal{L} & =\sum_{\text {all bins }} \mathcal{L}^{\prime}\left(N_{\text {obs }} \mid N_{\text {exp }}\right)-\mathcal{L}^{\prime}\left(N_{o b s} \mid N_{o b s}\right) \\
& =\sum_{\text {all bins }}-N_{\text {exp }}+N_{\text {obs }}+N_{\text {obs }} \ln \frac{N_{\text {exp }}}{N_{\text {obs }}}
\end{aligned}
$$

which Superman aficionados fluent in $\mathrm{LT}_{\mathrm{E}} \mathrm{X}$ will recognize immediately as Calel.

Once the normalized log likelihood has been calculated for an observed distribution, the oscillation (best-fit) hypothesis $H_{1}$ is compared to the null (no-disappearance) hypothesis $H_{0}$ by subtracting the likelihoods:

$$
\Delta \mathcal{L}=\mathcal{L}_{H_{1}}-\mathcal{L}_{H_{0}}
$$

This gives the log of the ratio of the probabilities, which can be exponentiated to yield the actual ratio of Poisson probabilities $B$ for the two hypotheses. For example, finding a $\Delta L=\ln 9 \sim 2.197$ leads to statements such as "The best-fit oscillation hypothesis is nine times more likely than the no-disappearance hypothesis", since $B=e^{\ln 9}=9$. The significance level $\alpha$ (see Section 7.3), with which $H_{0}$ is rejected, is related to the ratio $B$ by

$$
\alpha=1-\frac{B}{B+1} .
$$

The fixed-limit Texas Hold 'Em enthusiast will recognize the ratio $B$ as the betting odds. For example, if you hold an open-ended straight draw after the flop, there remain eight cards in the deck that will complete your straight. Eight of 47 unseen cards gives you a probability of $8 / 47=0.170$ to make your hand by the turn, and a probability of $1-(39 / 47 \times 38 / 46)=0.315$ to make your hand by the river. The odds against you improving by the end of the hand are $1 / 0.315-1=2.18: 1$. If you expect to put in less 
than $45 \%$ of the total pot by the end of the hand, you should stay in, if you believe the straight would beat anything your opponents are holding. You could impress your mates while you are raking their chips by saying "After the flop, the difference in log likelihood between one of you winning this hand and me winning this hand was $\Delta \mathcal{L}=0.78$ ". The author advises against such table talk, however. A simple "Nice hand" should suffice.

\section{C.2 Chi-squared approximation}

If the log likelihood surface in parameter space for a given fit is well-described by a parabola in both dimensions near the best-fit point, the errors on the measured parameters are Gaussian in nature, and one can safely assume the approximate definition

$$
-2 \mathcal{L}=\chi_{P}^{2}
$$

where $\mathcal{L}$ is as defined in Equation C.5 and $\chi_{P}^{2}$ is the usual Pearson's definition given in Equation C.9. This use of this approximation to define goodness-of-fit (using the reduced $\chi^{2}$ per degree of freedom) tends to break down in situations where there are low event rates in each bin of the fit. The standard Pearson's chi-squared value

$$
\chi^{2}=\frac{\left(N_{e x p}-N_{o b s}\right)^{2}}{N_{e x p}} .
$$

will deviate substantially from $-2 \mathcal{L}$ in situations where the number of events per bin is lower than about 10. For more details on the validity of this approximation and a nice treatment of the mean and variance for both $-2 \mathcal{L}$ and $\chi_{P}^{2}$, see Reference [68].

\section{C.3 Feldman-Cousins method}

In the case that the measurement errors on $\Delta m_{32}^{2}$ and $\sin ^{2} 2 \theta_{23}$ are not well-approximated as Gaussian (quadratic in the log likelihood) in both parameters, some degree of incorrect coverage of the parameter space by the confidence level contours is possible when using the $\chi^{2}$ approximation. In such cases, a unified approach has been suggested by Gary Feldman and Robert Cousins[69] as a classical approach providing proper coverage.

Typically, one uses the value of $\chi^{2}$ at the best fit point to determine the goodnessof-fit confidence level, while one uses the shape of the $\chi^{2}$ distribution in the parameter space to determine the confidence interval for both parameters. The Feldman-Cousins method is constructed in such a way as to decouple these two quoted confidence levels. 
As would be applied to the oscillation analysis of this work, the method for constructing the confidence level contours is as follows:

- Construct a grid of points in the desired oscillation parameter space $\left(\Delta m_{32 i}^{2}, \sin ^{2} 2 \theta_{23 j}\right)$.

- For each grid point, generate an ensemble of $N_{\text {exp }}$ pseudo-experiments having the specified values $\left(\Delta m_{32 i}^{2}, \sin ^{2} 2 \theta_{23 j}\right)$ as the input oscillation parameters. Each experiment contains $n_{\text {obs }}$ observed events drawn (consistent with a Poisson process having mean $\bar{n}_{o b s}$ ) from the expected reconstructed distribution of track momentum and direction. For a desired precision of $1 \%$ in the $\chi^{2}$ value determination, one requires $N_{\text {exp }}=10,000$ experiments.

- Each of these $N_{\text {exp }}$ experiments returns a best fit value $\left(\Delta m_{32 *}^{2}, \sin ^{2} 2 \theta_{23 *}\right)$ having a fit $\chi_{*}^{2}$, as well as $\chi_{i j}^{2}$ for the actual input oscillation parameters used at this grid point. Compute $\Delta \chi^{2}=\chi_{i j}^{2}-\chi_{*}^{2}$, which represents the "distance" in $\chi^{2}$ of the best fit point from the input point. In general, the fit performance will vary across the parameter space.

- Consider the resulting distribution of $N_{\exp }$ values of $\Delta \chi^{2}$. For any desired confidence level $\alpha$, determine the value $\Delta \chi^{2}{ }_{\alpha}$ for which the fraction $\alpha$ of experiments have $\Delta \chi^{2}<\Delta \chi_{\alpha}^{2}$.

- Perform the $\chi^{2}$ fit (on the same grid in parameter space) of the actual data to the expected distribution and obtain a $\chi^{2}$ surface with values $\chi_{A B}^{2}$ at each grid point, and best-fit value $\chi^{2}$ best.

- The value $\Delta \chi^{2}{ }_{\alpha}$ is in general different for each point $(i, j)$ in the parameter space. To construct the desired contour, one must plot the set of points on the grid for which $\chi_{A B}^{2}-\chi_{\text {best }}^{2}=\Delta \chi_{\alpha i j}^{2}$.

This procedure generates a contour shape that varies to some extent from the shape given by the simple $\chi^{2}$ approximation in C.2. The extent to which the simple $\chi^{2}$ contours deviate from an ellipse will determine the extent to which they undercover or overcover regions of the parameter space. The "banana" shape resulting from the $\chi^{2}$ approximation for this analysis at the current $1.27 \times 10^{20}$ protons on target exposure does improve with increased exposure. Statistically speaking, a representative bin in the fit will have a larger number of expected and observed events, bringing the log likelihood method into closer agreement with the standard chi-squared method. The contour shapes become more 
elliptical as a result. In the limit of large numbers, the two approaches are equivalent, and the approximation $\chi^{2}=-2 \mathcal{L}$ becomes an equality. 


\section{Appendix D}

\section{Selected event list}

Details of the Far Detector event selection criteria are found in Chapter 4. The events listed here were recorded between 20 May 2005 and 25 Feb 2006, the first year of NuMI running at the standard LE-10 position. Selected events are identified here by their Run and Snarl (a single time frame unit of detector activity) numbers. Only the 232 events found with the standard selection (that enter into the final analysis) are listed here. A + in the "Rock?" column indicates if the cut selection labeled an event as a rock muon. "Scan decision" labels are as follows: rock muon (RM), contained vertex charged current (CC), neutral current (NC), or no determination (XX). For events tagged as rock muons in the cut selection, "Entry" labels the position of the reconstructed track vertex: the outer $10 \mathrm{~cm}$ of any of the eight side faces (s), in the front two planes of SM 1 excluding the sides (f), or in the front two planes of SM 2 (SM gap entry) excluding the sides (g). Scanning "Notes" are included for some events: no track curvature measurement (NTCM), ambiguous hit timing information (AHTI), possible low-energy charged current (PLECC), or possible rock vertex interaction sending hadrons into detector (PRVH). Events that took place while the author was on shift in the control room are indicated with an asterisk in the "Notes" column.

All scan decisions here are those of the author. As noted in Section 4.2.3, M. Goodman, A. Allen, and S. Schwarzentraub all completed data scans that were used as consistency checks for the scan decisions shown here. M. Marshak and T. Mann have also

completed exhaustive scans of every data event, which were used to cross-check the decisions listed here. 


\begin{tabular}{|c|c|c|c|c|c|}
\hline Run & Snarl & Rock? & Scan decision & Entry & Notes \\
\hline 31725 & 5061 & + & $\mathrm{RM}$ & $f$ & \\
\hline 31732 & 76278 & + & $\mathrm{RM}$ & $\mathrm{s}$ & \\
\hline 31733 & 29491 & + & $\mathrm{RM}$ & $\mathrm{f}$ & \\
\hline 31737 & 21182 & & $\mathrm{CC}$ & & \\
\hline 31802 & 22039 & & $\mathrm{CC}$ & & \\
\hline 31807 & 36017 & & $\mathrm{NC}$ & & \\
\hline 31817 & 12454 & & $\mathrm{CC}$ & & \\
\hline 31831 & 117287 & + & RM & $\mathrm{f}$ & \\
\hline 31837 & 35946 & + & $\mathrm{RM}$ & $\mathrm{f}$ & \\
\hline 31873 & 66097 & + & $\mathrm{RM}$ & s & \\
\hline 31901 & 1589 & + & $\mathrm{RM}$ & $\mathrm{f}$ & \\
\hline 31929 & 27206 & + & $\mathrm{RM}$ & $\mathrm{f}$ & \\
\hline 31935 & 5819 & + & $\mathrm{RM}$ & $\mathrm{f}$ & \\
\hline 31950 & 20122 & & $\mathrm{CC}$ & & \\
\hline 31966 & 115059 & + & $\mathrm{RM}$ & $\mathrm{f}$ & \\
\hline 31976 & 3071 & + & $\mathrm{RM}$ & $\mathrm{s}$ & \\
\hline 31976 & 40731 & + & $\mathrm{RM}$ & $\mathrm{f}$ & \\
\hline 31984 & 131026 & + & $\mathrm{RM}$ & $\mathrm{s}$ & \\
\hline 31989 & 99675 & & $\mathrm{CC}$ & & \\
\hline 31994 & 86645 & + & $\mathrm{RM}$ & $\mathrm{f}$ & \\
\hline 32018 & 119778 & + & $\mathrm{RM}$ & $\mathrm{f}$ & \\
\hline 32027 & 113493 & + & $\mathrm{RM}$ & $\mathrm{f}$ & \\
\hline 32038 & 24517 & + & RM & $\mathrm{f}$ & \\
\hline 32110 & 870 & & $\mathrm{CC}$ & & \\
\hline 32131 & 22536 & + & $\mathrm{RM}$ & $\mathrm{s}$ & \\
\hline 32132 & 18426 & + & $\mathrm{RM}$ & $\mathrm{s}$ & \\
\hline 32136 & 86623 & + & $\mathrm{RM}$ & $\mathrm{f}$ & \\
\hline 32138 & 131909 & + & $\mathrm{RM}$ & $\mathrm{s}$ & \\
\hline 32162 & 65295 & + & $\mathrm{RM}$ & $\mathrm{f}$ & \\
\hline 32164 & 21332 & & $\mathrm{NC}$ & & PLECC \\
\hline 32177 & 44038 & + & $\mathrm{CC}$ & $\mathrm{s}$ & \\
\hline 32184 & 15911 & + & RM & $\mathrm{f}$ & \\
\hline
\end{tabular}

continued on next page... 


\begin{tabular}{|c|c|c|c|c|c|}
\hline Run & Snarl & Rock? & Scan decision & Entry & Notes \\
\hline 32189 & 51132 & + & $\mathrm{RM}$ & $f$ & \\
\hline 32189 & 82240 & + & $\mathrm{RM}$ & $\mathrm{f}$ & \\
\hline 32194 & 17810 & & $\mathrm{CC}$ & & \\
\hline 32194 & 70542 & + & RM & $\mathrm{s}$ & \\
\hline 32204 & 10601 & & RM & & \\
\hline 32211 & 91266 & + & RM & $\mathrm{f}$ & \\
\hline 32230 & 85094 & + & RM & $\mathrm{f}$ & \\
\hline 32481 & 10540 & + & $\mathrm{RM}$ & $\mathrm{f}$ & \\
\hline 32507 & 48547 & + & $\mathrm{RM}$ & $\mathrm{s}$ & \\
\hline 32518 & 9317 & + & $\mathrm{RM}$ & $\mathrm{f}$ & \\
\hline 32524 & 99646 & + & $\mathrm{RM}$ & $\mathrm{f}$ & \\
\hline 32566 & 44459 & & $\mathrm{CC}$ & & \\
\hline 32566 & 163460 & + & $\mathrm{RM}$ & $\mathrm{f}$ & \\
\hline 32566 & 297919 & + & $\mathrm{RM}$ & $\mathrm{f}$ & \\
\hline 32569 & 91668 & + & $\mathrm{XX}$ & S & NTCM, AHTI \\
\hline 32569 & 220950 & + & $\mathrm{RM}$ & $\mathrm{f}$ & \\
\hline 32578 & 328250 & + & $\mathrm{RM}$ & $\mathrm{s}$ & \\
\hline 32581 & 46155 & + & $\mathrm{RM}$ & $\mathrm{s}$ & \\
\hline 32583 & 60823 & + & $\mathrm{RM}$ & $\mathrm{s}$ & \\
\hline 32586 & 97587 & + & $\mathrm{RM}$ & $\mathrm{f}$ & \\
\hline 32596 & 268585 & & $\mathrm{CC}$ & & \\
\hline 32599 & 206826 & + & $\mathrm{RM}$ & $\mathrm{f}$ & \\
\hline 32608 & 239020 & + & $\mathrm{RM}$ & $\mathrm{s}$ & \\
\hline 32611 & 250486 & + & $\mathrm{RM}$ & $\mathrm{f}$ & \\
\hline 32611 & 286668 & + & $\mathrm{RM}$ & $\mathrm{f}$ & \\
\hline 32617 & 138857 & + & $\mathrm{RM}$ & $\mathrm{f}$ & \\
\hline 32617 & 177092 & + & $\mathrm{RM}$ & $\mathrm{f}$ & \\
\hline 32617 & 208294 & & $\mathrm{CC}$ & & \\
\hline 32617 & 214567 & & $\mathrm{CC}$ & & \\
\hline 32623 & 290657 & + & $\mathrm{RM}$ & $\mathrm{f}$ & \\
\hline 32626 & 22094 & + & $\mathrm{RM}$ & $\mathrm{f}$ & \\
\hline 32629 & 48200 & & $\mathrm{CC}$ & & \\
\hline
\end{tabular}

continued on next page... 


\begin{tabular}{|c|c|c|c|c|c|}
\hline Run & Snarl & Rock? & Scan decision & Entry & Notes \\
\hline 32632 & 113763 & + & $\mathrm{RM}$ & $f$ & \\
\hline 32638 & 265876 & + & $\mathrm{RM}$ & $\mathrm{f}$ & \\
\hline 32644 & 252628 & + & $\mathrm{RM}$ & $\mathrm{f}$ & \\
\hline 32654 & 88657 & + & RM & $\mathrm{f}$ & \\
\hline 32663 & 170496 & + & RM & $\mathrm{f}$ & \\
\hline 32666 & 106294 & + & RM & $\mathrm{f}$ & \\
\hline 32672 & 221246 & & $\mathrm{CC}$ & & \\
\hline 32678 & 132148 & + & RM & $\mathrm{f}$ & \\
\hline 32687 & 315119 & + & $\mathrm{RM}$ & $\mathrm{f}$ & \\
\hline 32690 & 288197 & + & $\mathrm{RM}$ & $\mathrm{f}$ & \\
\hline 32713 & 276953 & + & $\mathrm{RM}$ & $\mathrm{f}$ & $*$ \\
\hline 32713 & 374331 & + & $\mathrm{RM}$ & $\mathrm{s}$ & \\
\hline 32719 & 10298 & + & $\mathrm{RM}$ & $\mathrm{s}$ & \\
\hline 32725 & 199664 & + & $\mathrm{RM}$ & $\mathrm{s}$ & \\
\hline 32725 & 270017 & & $\mathrm{CC}$ & & $*$ \\
\hline 32725 & 412692 & + & $\mathrm{CC}$ & $\mathrm{s}$ & \\
\hline 32728 & 188242 & + & $\mathrm{RM}$ & $\mathrm{f}$ & $*$ \\
\hline 32731 & 17951 & + & $\mathrm{RM}$ & $\mathrm{s}$ & $*$ \\
\hline 32731 & 83850 & + & $\mathrm{RM}$ & $\mathrm{f}$ & \\
\hline 32734 & 363441 & & $\mathrm{CC}$ & & $*$ \\
\hline 32737 & 112940 & + & $\mathrm{RM}$ & $\mathrm{f}$ & \\
\hline 32740 & 39513 & + & $\mathrm{RM}$ & $\mathrm{s}$ & \\
\hline 32746 & 297058 & & $\mathrm{CC}$ & & \\
\hline 32750 & 237351 & + & $\mathrm{RM}$ & $\mathrm{s}$ & \\
\hline 32750 & 269847 & + & $\mathrm{RM}$ & $\mathrm{f}$ & \\
\hline 32782 & 196166 & + & $\mathrm{RM}$ & $\mathrm{s}$ & \\
\hline 32782 & 324564 & + & $\mathrm{RM}$ & $\mathrm{f}$ & \\
\hline 32785 & 194417 & + & $\mathrm{RM}$ & $\mathrm{s}$ & \\
\hline 32785 & 324035 & + & $\mathrm{RM}$ & $\mathrm{f}$ & \\
\hline 32791 & 179534 & + & $\mathrm{RM}$ & $\mathrm{s}$ & \\
\hline 32799 & 298266 & + & $\mathrm{RM}$ & $\mathrm{s}$ & \\
\hline 32802 & 101959 & + & RM & $\mathrm{s}$ & \\
\hline
\end{tabular}

continued on next page... 


\begin{tabular}{|c|c|c|c|c|c|}
\hline Run & Snarl & Rock? & Scan decision & Entry & Notes \\
\hline 32802 & 137929 & & $\mathrm{CC}$ & & \\
\hline 32805 & 20380 & & $\mathrm{CC}$ & & \\
\hline 32808 & 42050 & & $\mathrm{CC}$ & & \\
\hline 32811 & 231524 & + & RM & $\mathrm{s}$ & \\
\hline 32814 & 104391 & + & $\mathrm{RM}$ & $\mathrm{f}$ & \\
\hline 32898 & 35249 & + & $\mathrm{NC}$ & $\mathrm{s}$ & * \\
\hline 32898 & 160233 & + & RM & $\mathrm{f}$ & \\
\hline 32901 & 156899 & + & $\mathrm{RM}$ & $\mathrm{s}$ & \\
\hline 32901 & 220947 & + & $\mathrm{RM}$ & $\mathrm{f}$ & \\
\hline 32907 & 79715 & + & $\mathrm{RM}$ & $\mathrm{s}$ & \\
\hline 32907 & 286214 & + & $\mathrm{RM}$ & s & \\
\hline 32910 & 78755 & + & $\mathrm{RM}$ & $\mathrm{s}$ & \\
\hline 32951 & 119146 & + & $\mathrm{RM}$ & $\mathrm{s}$ & \\
\hline 32951 & 140424 & & $\mathrm{CC}$ & & \\
\hline 32973 & 113263 & + & $\mathrm{RM}$ & $\mathrm{f}$ & $*$ \\
\hline 32976 & 135130 & + & $\mathrm{RM}$ & $\mathrm{f}$ & * \\
\hline 32997 & 161433 & + & $\mathrm{RM}$ & $\mathrm{f}$ & $*$ \\
\hline 33006 & 131599 & + & $\mathrm{RM}$ & $\mathrm{f}$ & $*$ \\
\hline 33006 & 144786 & & $\mathrm{CC}$ & & $*$ \\
\hline 33014 & 88699 & + & $\mathrm{RM}$ & $\mathrm{s}$ & $*$ \\
\hline 33017 & 123229 & + & $\mathrm{RM}$ & $\mathrm{s}$ & \\
\hline 33017 & 132080 & + & $\mathrm{RM}$ & $\mathrm{f}$ & \\
\hline 33020 & 314099 & + & RM & $\mathrm{f}$ & \\
\hline 33023 & 48237 & & $\mathrm{CC}$ & & \\
\hline 33023 & 114628 & & $\mathrm{CC}$ & & \\
\hline 33035 & 29019 & + & $\mathrm{CC}$ & $\mathrm{f}$ & $*$ \\
\hline 33035 & 61403 & & $\mathrm{CC}$ & & \\
\hline 33080 & 124121 & + & $\mathrm{RM}$ & $\mathrm{s}$ & \\
\hline 33080 & 237747 & + & $\mathrm{RM}$ & $\mathrm{f}$ & \\
\hline 33086 & 141017 & & $\mathrm{CC}$ & & \\
\hline 33086 & 236577 & + & $\mathrm{RM}$ & $\mathrm{f}$ & \\
\hline 33089 & 14425 & + & RM & $\mathrm{S}$ & \\
\hline
\end{tabular}

continued on next page... 


\begin{tabular}{|c|c|c|c|c|c|}
\hline Run & Snarl & Rock? & Scan decision & Entry & Notes \\
\hline 33096 & 86076 & + & $\mathrm{RM}$ & $f$ & \\
\hline 33102 & 129256 & + & $\mathrm{RM}$ & $\mathrm{s}$ & \\
\hline 33108 & 141257 & & $\mathrm{CC}$ & & \\
\hline 33119 & 96978 & & RM & & \\
\hline 33126 & 55984 & & $\mathrm{CC}$ & & \\
\hline 33126 & 105390 & & $\mathrm{CC}$ & & \\
\hline 33129 & 159428 & + & $\mathrm{RM}$ & $\mathrm{f}$ & \\
\hline 33135 & 142152 & + & $\mathrm{RM}$ & $\mathrm{s}$ & \\
\hline 33141 & 202277 & + & $\mathrm{RM}$ & $\mathrm{f}$ & \\
\hline 33156 & 57894 & + & $\mathrm{RM}$ & $\mathrm{f}$ & \\
\hline 33156 & 83372 & + & $\mathrm{RM}$ & $\mathrm{f}$ & \\
\hline 33156 & 254861 & + & $\mathrm{RM}$ & $\mathrm{s}$ & \\
\hline 33159 & 192238 & + & $\mathrm{CC}$ & $\mathrm{s}$ & \\
\hline 33162 & 263487 & + & $\mathrm{RM}$ & $\mathrm{s}$ & \\
\hline 33165 & 149587 & + & $\mathrm{RM}$ & $\mathrm{f}$ & \\
\hline 33165 & 236419 & & $\mathrm{CC}$ & & \\
\hline 33168 & 128002 & & $\mathrm{CC}$ & & \\
\hline 33168 & 130025 & + & $\mathrm{RM}$ & $\mathrm{s}$ & \\
\hline 33178 & 222451 & + & $\mathrm{RM}$ & $\mathrm{f}$ & \\
\hline 33181 & 125812 & + & $\mathrm{RM}$ & $\mathrm{f}$ & \\
\hline 33184 & 176837 & + & $\mathrm{NC}$ & $\mathrm{s}$ & PRVH \\
\hline 33184 & 235339 & + & $\mathrm{NC}$ & $\mathrm{f}$ & PRVH, NTCM \\
\hline 33187 & 299514 & + & RM & $\mathrm{f}$ & \\
\hline 33190 & 190400 & + & $\mathrm{RM}$ & $\mathrm{s}$ & \\
\hline 33227 & 116202 & & $\mathrm{RM}$ & & \\
\hline 33253 & 246336 & + & $\mathrm{RM}$ & $\mathrm{s}$ & \\
\hline 33256 & 156835 & & $\mathrm{RM}$ & & \\
\hline 33256 & 157769 & & $\mathrm{CC}$ & & \\
\hline 33262 & 63635 & & $\mathrm{CC}$ & & \\
\hline 33262 & 150815 & + & $\mathrm{RM}$ & $\mathrm{f}$ & \\
\hline 33262 & 196148 & + & $\mathrm{RM}$ & $\mathrm{s}$ & \\
\hline 33277 & 9403 & + & RM & $\mathrm{f}$ & \\
\hline
\end{tabular}

continued on next page... 


\begin{tabular}{|c|c|c|c|c|c|}
\hline Run & Snarl & Rock? & Scan decision & Entry & Notes \\
\hline 33277 & 59748 & + & $\mathrm{RM}$ & $\mathrm{f}$ & \\
\hline 33277 & 119552 & + & $\mathrm{RM}$ & $\mathrm{s}$ & \\
\hline 33277 & 128849 & + & $\mathrm{RM}$ & $\mathrm{f}$ & \\
\hline 33280 & 44825 & + & $\mathrm{RM}$ & $\mathrm{f}$ & \\
\hline 33280 & 74826 & + & $\mathrm{RM}$ & $\mathrm{f}$ & \\
\hline 33280 & 106657 & + & $\mathrm{RM}$ & $\mathrm{f}$ & \\
\hline 33280 & 190226 & + & $\mathrm{CC}$ & $\mathrm{s}$ & \\
\hline 33288 & 153260 & & $\mathrm{RM}$ & & \\
\hline 33288 & 169146 & + & $\mathrm{RM}$ & $\mathrm{f}$ & \\
\hline 33291 & 212058 & & $\mathrm{CC}$ & & \\
\hline 33297 & 197186 & + & $\mathrm{RM}$ & $\mathrm{f}$ & \\
\hline 33297 & 212505 & & $\mathrm{CC}$ & & \\
\hline 33297 & 278394 & & $\mathrm{NC}$ & & PLECC \\
\hline 33412 & 163528 & + & $\mathrm{RM}$ & $\mathrm{f}$ & \\
\hline 33415 & 15655 & + & $\mathrm{CC}$ & $\mathrm{g}$ & \\
\hline 33443 & 216590 & + & $\mathrm{RM}$ & $\mathrm{f}$ & \\
\hline 33446 & 242349 & + & $\mathrm{RM}$ & $\mathrm{f}$ & \\
\hline 33446 & 247257 & + & $\mathrm{RM}$ & $\mathrm{s}$ & \\
\hline 33449 & 92381 & + & $\mathrm{RM}$ & $\mathrm{s}$ & \\
\hline 33452 & 48800 & + & $\mathrm{RM}$ & $\mathrm{f}$ & \\
\hline 33452 & 282767 & + & $\mathrm{CC}$ & $\mathrm{g}$ & \\
\hline 33455 & 210774 & + & RM & $\mathrm{s}$ & \\
\hline 33455 & 242866 & + & RM & $\mathrm{s}$ & \\
\hline 33458 & 300892 & + & $\mathrm{RM}$ & $\mathrm{s}$ & \\
\hline 33474 & 234680 & + & $\mathrm{RM}$ & $\mathrm{f}$ & \\
\hline 33477 & 183339 & + & $\mathrm{NC}$ & $\mathrm{s}$ & PRVH \\
\hline 33480 & 51501 & & $\mathrm{CC}$ & & \\
\hline 33486 & 200660 & + & $\mathrm{RM}$ & $\mathrm{f}$ & \\
\hline 33489 & 97768 & + & $\mathrm{RM}$ & $\mathrm{s}$ & \\
\hline 33495 & 107302 & & $\mathrm{CC}$ & & \\
\hline 33502 & 247756 & + & RM & s & \\
\hline 33508 & 102700 & + & RM & $\mathrm{s}$ & \\
\hline
\end{tabular}

continued on next page... 


\begin{tabular}{|c|c|c|c|c|c|}
\hline \hline Run & Snarl & Rock? & Scan decision & Entry & Notes \\
\hline 33508 & 291595 & & $\mathrm{CC}$ & & \\
33514 & 84150 & + & $\mathrm{RM}$ & $\mathrm{f}$ & \\
33517 & 125233 & & $\mathrm{NC}$ & & PLECC \\
33520 & 200884 & + & $\mathrm{RM}$ & $\mathrm{f}$ & $*$ \\
33542 & 28508 & + & $\mathrm{RM}$ & $\mathrm{s}$ & \\
33542 & 256716 & + & $\mathrm{RM}$ & $\mathrm{f}$ & \\
33542 & 297277 & + & $\mathrm{RM}$ & $\mathrm{s}$ & \\
33545 & 34221 & + & $\mathrm{RM}$ & $\mathrm{f}$ & \\
33545 & 81516 & + & $\mathrm{RM}$ & $\mathrm{s}$ & \\
33545 & 224964 & + & $\mathrm{NC}$ & $\mathrm{g}$ & \\
33552 & 63762 & + & $\mathrm{RM}$ & $\mathrm{s}$ & \\
33598 & 37909 & + & $\mathrm{RM}$ & $\mathrm{f}$ & \\
33598 & 89669 & + & $\mathrm{RM}$ & $\mathrm{f}$ & \\
33598 & 150479 & + & $\mathrm{RM}$ & $\mathrm{f}$ & \\
33606 & 118058 & + & $\mathrm{RM}$ & $\mathrm{s}$ & \\
33613 & 182466 & & $\mathrm{RM}$ & & \\
33613 & 224589 & + & $\mathrm{RM}$ & $\mathrm{f}$ & \\
33616 & 21721 & + & $\mathrm{RM}$ & $\mathrm{s}$ & \\
33616 & 229054 & + & $\mathrm{RM}$ & $\mathrm{f}$ & \\
33619 & 243451 & & $\mathrm{RM}$ & & \\
33619 & 256665 & + & $\mathrm{RM}$ & $\mathrm{f}$ & \\
33649 & 99469 & + & $\mathrm{RM}$ & $\mathrm{f}$ & \\
33649 & 125847 & + & $\mathrm{RM}$ & $\mathrm{s}$ & \\
33668 & 140012 & + & $\mathrm{RM}$ & $\mathrm{f}$ & \\
33671 & 186178 & + & $\mathrm{RM}$ & $\mathrm{f}$ & \\
33698 & 41686 & + & $\mathrm{RM}$ & $\mathrm{s}$ & \\
33698 & 83111 & + & $\mathrm{RM}$ & $\mathrm{s}$ & \\
33704 & 97381 & + & $\mathrm{RM}$ & $\mathrm{f}$ & \\
33713 & 221282 & + & $\mathrm{RM}$ & $\mathrm{s}$ & \\
33722 & 117010 & + & $\mathrm{R}$ & $\mathrm{f}$ & \\
33722 & 168880 & + & $\mathrm{R}$ & \\
33726 & 120441 & + & $\mathrm{R}$ & \\
\hline
\end{tabular}

continued on next page... 


\begin{tabular}{|c|c|c|c|c|c|}
\hline \hline Run & Snarl & Rock? & Scan decision & Entry & Notes \\
\hline 33726 & 169741 & + & RM & $\mathrm{s}$ & \\
33741 & 19868 & + & RM & $\mathrm{f}$ & \\
33741 & 74910 & & $\mathrm{CC}$ & & \\
33750 & 116279 & + & $\mathrm{RM}$ & $\mathrm{f}$ & \\
33750 & 165182 & + & $\mathrm{RM}$ & $\mathrm{f}$ & \\
33750 & 207983 & & $\mathrm{CC}$ & & \\
33753 & 46768 & & $\mathrm{CC}$ & & \\
33756 & 74628 & + & $\mathrm{RM}$ & $\mathrm{f}$ & \\
\hline \hline
\end{tabular}

Table D.1: Comparison of three selection decisions for all events used in the oscillation analysis. 


\section{References}

[1] D. G. Michael et al., Observation of muon neutrino disappearance with the MINOS detectors and the NuMI neutrino beam, Phys. Rev. Lett. 97, 191801 (2006).

[2] Y. Ashie et al., A Measurement of Atmospheric Neutrino Oscillation Parameters by Super-Kamiokande I, Phys. Rev. D 71, 112005 (2005).

[3] J. Hosaka et al., Three flavor neutrino oscillation analysis of atmospheric neutrinos in Super-Kamiokande, Phys. Rev. D 74, 032002 (2006).

[4] E. Aliu et al., Evidence for muon neutrino oscillation in an accelerator-based experiment, Phys. Rev. Lett. 94, 081802 (2005).

[5] M. H. Ahn et al., Measurement of Neutrino Oscillation by the K2K Experiment, Phys. Rev. D 74, 072003 (2006).

[6] Nova, The Ghost Particle, first aired on PBS, 21 February 2006.

[7] Jeopardy, Episode 147, first aired 04 April 2006.

[8] F. Reines and C. L. Cowan, Jr., Detection of the Free Neutrino, Phys. Rev. 92, 830 (1953).

[9] F. Reines and C. L. Cowan, Jr., Free Antineutrino Absorption Cross Section. I. Measurement of the Free Antineutrino Absorption Cross Section by Protons, Phys. Rev. 113, 273 (1959).

[10] R. Davis, Jr., D. S. Harmer, and K. C. Hoffman, Search for neutrinos from the sun, Phys. Rev. Lett. 20, 1205 (1968).

[11] J. N. Bahcall and N. A. Bahcall, Present status of the theoretical predictions for the ${ }^{36}$ Cl solar-neutrino experiment, Phys. Rev. Lett. 20, 1209 (1968).

[12] H. A. Bethe, Possible Explanation of the Solar-Neutrino Puzzle, Phys. Rev. Lett. 56, 1305 (1986). 
[13] W. W. M. Allison et al., Measurement of the atmospheric neutrino flavor composition in Soudan 2, Phys. Lett. B 391, 491 (1997), arXiv:hep-ex/9611007.

[14] J. G. Learned, The Atmospheric Neutrino Anomaly: Muon Neutrino Disappearance, arXiv:hep-ex/0007056v1 (2000).

[15] E. L. Wright, Neutrinos as Dark Matter. 21 Sep. 1998. http://www.astro.ucla.edu/w̃wright/neutrinos.html.

[16] S. W. Allen, R. W. Schmidt, and S. L. Bridle, A Preference for a non-zero neutrino mass from cosmological data, Mon. Not. Roy. Astron. Soc. 346, 593 (2003), arXiv:astro-ph/0306386.

[17] A. G. Dias et al., Neutrino Decay and Neutrinoless Double Beta Decay in a 3-3-1 Model, Phys. Rev. D 72, 035006 (2005), arXiv:hep-ph/0503014.

[18] G. Barenboim et al., Quantum Decoherence and Neutrino Data, Nucl. Phys. B 758, 90 (2006), arXiv:hep-ph/0603028.

[19] A. de Gouvea and Y. Grossman, A Three-Flavor, Lorentz-Violating Solution to the LSND Anomaly?, Phys. Rev. D 74, 093008 (2006), arXiv:hep-ph/0602237.

[20] X.-Q. Li, Y. Liu, and Z.-T. Wei, Neutrino Decay as a possible interpretation to the MiniBooNE observation with unparticle scenario, arXiv:hep-ph/0707.2285.

[21] M. Schwartz, Feasibility of using high-energy neutrinos to study the weak interactions, Phys. Rev. Lett. 4, 306 (1960).

[22] G. Danby et al., Observation of High-Energy Neutrino Reactions and the Existence of Two Kinds of Neutrinos, Phys. Rev. Lett. 9, 36 (1962).

[23] P. Lipari, M. Lusignoli, and F. Sartogo, The neutrino cross section and upward going muons, Phys. Rev. Lett 74, 4384 (1995), arXiv:hep-ph/9411341.

[24] K. A. Lefler, The Total Cross-Section for Muon-Neutrino Electron Elastic Scattering, Ph.D. thesis, University of Maryland College Park (1981).

[25] J. D. Bjorken, A long baseline mu-neutrino disappearance experiment for Fermilab, New Directions in Neutrino Physics at Fermilab, 14-16 September 1998, p.259.

[26] M. Aglietta et al., CNGS beam monitor with the LVD detector, Nucl. Instrum. Meth. A 516, 96 (2004), arXiv:hep-ex/0304018.

[27] R. Acquafredda et al., First events from the CNGS neutrino beam detected in the OPERA experiment, New J. Phys. 8, 303 (2006), arXiv:hep-ex/0611023. 
[28] H. Menghetti for the LVD Collaboration, First results of the CNGS beam monitor with LVD, LNGS, 22 August 2006, http://proj-cngs.web.cern.ch/projcngs/PDF_files/LVD_CNGS22AugLNGS.pdf.

[29] L. Perasso, The CNGS beam and the Borexino events in October 2006, http://www.ge.infn.it/borexino/CNGS/CNGS_lperep_jan2007.pdf, (2007).

[30] V. Gribov and B. Pontecorvo, Neutrino astronomy and lepton charge, Phys. Lett. B 28, 493 (1969).

[31] B. Pontecorvo, Zh. Exp. i Teor. Fiz. 34 (1958).

[32] B. Pontecorvo, J. Expl. Theoret. Phys. 53, 1717 (1967).

[33] A. K. Mann and H. Primakoff, Neutrino oscillations and the number of neutrino types, Phys. Rev. D 15, 655 (1977).

[34] The MINOS Collaboration, P-875: A Long-baseline Neutrino Oscillation Experiment at Fermilab, Chapter 8: Physics Signatures, February 1995, FERMILABProposal-0875.

[35] The MINOS Collaboration, The MINOS Detectors Technical Design Report, Chapter 1: Introduction, October 1998, NuMI-L-337.

[36] K. S. Hirata et al., Experimental study of the atmospheric neutrino flux, Phys. Lett. B 205, 416 (1988).

[37] H.-J. Trost, Search for $\nu_{\mu} \rightarrow \nu_{\tau}$ oscillations using the far detector and the rock around it, PDK Internal Collaboration Note.

[38] M. Martens, Target and Horn Configuration for SNuMI, NOvA, and MINERvA, Fermilab Beams Document 2392-v1.

[39] N. Tagg, ToF Paper Discussion, MINOS Document 2919-v1.

[40] M. Kordosky, Hadronic Interactions in the MINOS Detectors, Ph.D. thesis, University of Texas at Austin (2004).

[41] P. Vahle, Electromagnetic Interactions in the MINOS Detectors, Ph.D. thesis, University of Texas at Austin (2004).

[42] P. A. Symes, Preliminary Measurement of Neutrino Oscillation Parameters By NuMI/MINOS And Calibration Studies For Improving This Measurement, Ph.D. thesis, University of Sussex (2005).

[43] N. Felt et al, MINOS Far Detector Electronics - User's Manual, NuMI Document 901 (2002). 
[44] D. G. Michael et al, The MINOS Detectors, MINOS Document 1967-v15. To be submitted to Nucl. Instrum. Meth.

[45] A. Fasso et al. the official FLUKA site, online manual 19 Jul. 2007. http://www.fluka.org/manual/Online.shtml.

[46] Z. Pavlovic. NuMI Beam Monte Carlo Code 01 Nov. 2006. http://www.hep.utexas.edu/ zarko/wwwgnumi/v19/.

[47] J. Apostolakis. GEANT - Detector Description and Simulation Tool. 07 May 2003. http://wwwasd.web.cern.ch/wwwasd/geant/.

[48] R. Hatcher. A status report on GMINOS (circa 2003-01-30). 31 Jan. 2003. http://home.fnal.gov/r̃hatcher/gminos/review_and_status_030131.html.

[49] R. Hatcher. The Labyrinth. 06 Jul. 2007. http://wwwnumi.fnal.gov/offline_software/labyrinth/.

[50] H. Gallagher. NEUGEN. 18 Jan. 2006. http://minos.phy.tufts.edu/gallag/neugen/.

[51] F. Yumiceva, GNUMI v.18 FLUKA Fluxes, MINOS Document 1137-v1.

[52] A. Marino, Notes on GNuMI v19 Flux Files, MINOS Document 2153-v1.

[53] The MINOS Collaboration, The MINOS Detectors Technical Design Report, Chapter 9: Software and Computing, October 1998, NuMI-L-337.

[54] W.-M. Yao et al., Review of Particle Physics, J. Phys. Lett. G 33, 1 (2006).

[55] S. Kopp et al., Constraining the Beam Neutrino MC Flux Using the MINOS ND Data, MINOS Document 2965-v1.

[56] C. Andreopoulos, MuELoss: C++ software package for computing $\mu^{ \pm}$energy losses in the energy range up to $10 \mathrm{TeV}$, NuMI-note-COMP-1001, 05 Jan. 2004.

[57] K. Ruddick, private communication, 21 Jun. 2007.

[58] S. M. Kasahara, A Study of Cosmic Ray Composition in the Knee Region using Multiple Muon Events in the Soudan 2 Detector, Ph.D. thesis, University of Minnesota (1997).

[59] M. Ishitsuka, Validation of spectrum (WECC version), MINOS Document 1655-v5.

[60] M. Marshak, Charged Current Event Analysis By Scanning, MINOS Document $1512-\mathrm{v} 1$

[61] W. A. Mann, Scan Rules for Selecting CC Candidates Amongst Short-Track Events, MINOS Document 2980-v3 
[62] D. Petyt, Description of simple likelihood-based PID, MINOS Document 2736-v1.

[63] T. H. Fields, Maximum Detectable Momentum in the MINOS Far Detector, MINOS Document 1511-v1.

[64] M. Goodman, The Maximum Detectable Momentum for cosmic ray muons in the MINOS far detector, Contribution to the 30th International Cosmic Ray Conference, Merida Mexico 2007, arXiv:hep-ex/0706.0539v1.

[65] J. K. Nelson, Notes on the $N / F$ relative normalization in the $C C$ analysis, MINOS Document 2106-v4.

[66] A. D. Marino, MDC III revealed, MINOS Document 1820-v2.

[67] A. M. McGowan and G. Giurgiu, Oscillation analysis sensitivity of rock muons in the MINOS far detector, MINOS Document 2184.

[68] J. G. Heinrich, The Log Likelihood Ratio of the Poisson Distribution for Small $\mu$, CDF/MEMO/CDF/CDFR/5718, Version 2, 11 December 2001.

[69] G. J. Feldman and R. D. Cousins, Unified approach to the classical statistical analysis of small signals, Phys. Rev. D. 57, 3873 (1998). 
A new detector!

Scores of neutrinos fall in -

The sound of laughter

Miniscule specters

Come and go without a trace:

Just like my paychecks

Far below the grass

A light blinks, a counter clicks!

The physicist sleeps

Why too few, no mu?

Past the mine, particles flew

neutrino, et tu?

Mysterious specks

Billions pass through me each day:

Now my skin itches

-David S. Jacque 
The author currently resides in Rochester, New York with his wife, Sarah. He teaches physics as a Visiting Assistant Professor at St. John Fisher College. He holds a Visiting Scientist position at the University of Rochester and a Guest Research appointment at Argonne National Laboratory.

This document was typeset in 11-point Helvetica font using $\mathrm{LTT}_{\mathrm{E} X} \mathrm{Xe}$. 